\author{
Universidade de São Paulo \\ Instituto de Física
}

\title{
Dipolos de cor e interações a altas energias
}

Maria Simone Kugeratski Souza

Orientador: Prof. Dr. Fernando Silveira Navarra Co-Orientador: Prof. Dr. Victor Paulo Barros Gonçalves

Tese de doutorado apresentada ao Instituto de Física para a obtenção do título de Doutor em Ciências

Banca Examinadora:

Prof. Dr. Fernando Silveira Navarra (orientador) (IFUSP)

Prof $^{a}$. Dra. Ivone Freire Mota Albuquerque (IFUSP)

Prof. Dr. Adriano Antonio Natale (IFT/UNESP)

Prof. Dr. Álvaro Leonardi Ayala Filho (UFPel)

Prof. Dr. Sérgio Szpigel (MACKENZIE)

São Paulo 


\section{FICHA CATALOGRÁFICA \\ Preparada pelo Serviço de Biblioteca e Informação \\ do Instituto de Física da Universidade de São Paulo}

Souza, Maria Simone Kugeratski

Dipolos de cor e interações a altas energias.

São Paulo, 2007.

Tese (Doutorado) - Universidade de São Paulo.

Instituto de Física. Departamento de Física

Experimental.

Orientador: Prof. Dr. Fernando Silveira Navarra

Área de Concentração: Física

Unitermos: 1. Física de partículas; 2.

Fenomenologia

3. Saturação de Pártons

USP/IF/SBI-029/2007 
À minha familia, Ao Edson. 


\section{Agradecimentos}

- Agradeço aos professores Fernando Navarra e Victor Gonçalves, pela orientação e amizade.

- Agradeço à minha família pelo apoio ao longo de todos esses anos, aos sobrinhos Marise, Júnior e Eduardo pelo encanto.

- Agradeço ao Edson por estar sempre ao meu lado.

- Agradeço a todos professores, colegas do corredor e amigos do Grhafite: Marina Nielsen, Robillota e Celso Lima, David, Diogo, Gabriel, Ricardo, Arthur, Pati, Dani, Babi, Celso, Rafael, pela compania agradável de todos esses anos.

- Agradeço ao pessoal da secretaria, ao Paulo pela sua ajuda paciente no scaner, à Olga pelos cafezinhos e por ter proporcionado um ambiente descontraído.

- Agradeço ao pessoal da Universidade Federal de Pelotas pela acolhida durante meus estágios com o professor Victor Gonçalves.

- Agradeço ao professor Edmond Iancu por ter proporcionado a realização de um estágio em Saclay em seu grupo de pesquisa.

- Agradeço as meninas da república Giselle e Heily pela compania.

- Agradeço a Kelly, Regina, Andréa, Émerson, Marcelo, Claúdia, Marilda, Juliano, Marlete, pela amizade. 
- Agradeço a Lilian pela amizade e pela hospedagem nesse final de doutorado.

- Agradeço de modo geral a todos professores e colegas que contribuiram para minha formação ao longos de todos esses anos no Instituto de Física.

- Agradeço ao Odair e ao Rodrigo pela assistência no linux.

- Agradeço ao CNPq pelos primeiros seis meses de bolsa e a FAPESP pelo apoio financeiro no restante do doutorado. 


\section{Resumo}

A altas energias o núcleon (ou o núcleo) pode ser visto como um sistema denso de glúons, o condensado de vidro de cor. Neste trabalho estudamos a interação deste sistema com dipolos de cor. Esta interação é representada pela seção de choque dipoloalvo, a qual é modelada. Utilizamos alguns dos modelos propostos na literatura para descrever os dados do HERA e do RHIC e propusemos uma nova parametrização que é capaz de descrever estes dados simultaneamente, demonstrando a propriedade de universalidade do condensado de vidro de cor. Generalizamos o modelo proposto por Iancu, Itakura e Munier para o caso nuclear e o aplicamos ao estudo de espalhamento elétron-núcleo. Estimamos a influência dos efeitos de saturação nos observáveis que serão medidos no futuro eRHIC. Concluímos entre outras coisas, que uma grande fração dos eventos observados serão do tipo difrativo. Finalmente usamos a idéia de dipolo de cor para estudar a seção de choque charmonium-hádron. 


\section{Abstract}

At high energies a nucleus (and even a nucleon) becomes a dense gluonic system called the color glass condensate. In this work we study the interaction of this dense system with color dipoles. This interaction is represented by the dipole-target cross section, which has to be modeled. We have used, without success, some of the existing models to try to reproduce the data obtained at HERA and RHIC. It was not possible to obtain a global agreement between the models and the two sets of data. We have then proposed a different parametrization of the dipole cross section, which is now compatible with all the available data. In the case of deep inelastic scattering, we have extended the Iancu-Itakura-Munier model to nuclear targets and made predictions for observables to be measured in the future electron-ion collider eRHIC. Among other things, we have concluded that a large fraction of the events will be of the diffractive type. Finally, we have used the color dipole approach to build a simple model for the charmonium-hadron interaction at high energies. 


\section{Sumário}

$\begin{array}{ll}\text { Introdução } & 1\end{array}$

1 Espalhamento inelástico profundo 5

1.1 A cromodinâmica quântica . . . . . . . . . . . . . . 5

1.2 O espalhamento inelástico profundo . . . . . . . . . . 10

1.3 O modelo a pártons . . . . . . . . . . . . . . . . 16

1.4 As equações de DGLAP . . . . . . . . . . . . . . . 17

1.5 A dinâmica DGLAP para pequeno $x \ldots \ldots \ldots . \ldots \ldots$

1.6 Saturação . . . . . . . . . . . . . . . . . . 36

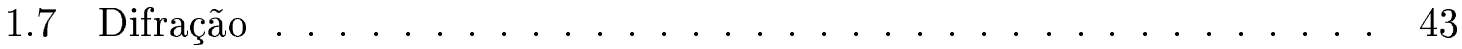

$\begin{array}{lll}2 & \text { Dipolos de cor } & 47\end{array}$

2.1 DIS na representação de dipolos de cor . . . . . . . . . . . 47

2.1.1 A fatorização $k_{T}$ e a seção de choque de dipolo . . . . . . . . . 49

2.1 .2 Análise qualitativa . . . . . . . . . . . . 56

2.2 Espalhamento difrativo . . . . . . . . . . . . . . . . 60

2.2 .1 A cinemática do DDIS . . . . . . . . . . . . 60

2.2 .2 Funções de estrutura difrativa . . . . . . . . . . . . . 61

2.2.3 O modelo de troca de dois gluons . . . . . . . . . . 62

3 Equações não lineares de evolução

3.1 Introdução . . . . . . . . . . . . . . . . 73 
3.2 A equação de Balitsky-Kovchegov . . . . . . . . . . . . . 75

3.3 A equação de BFKL no limite de saturação . . . . . . . . . . . . 80

3.4 Scaling geométrico na equação de BFKL . . . . . . . . . . . . 84

3.5 Modelos fenomenológicos . . . . . . . . . . . . . . . 87

4 Universalidade dos modelos fenomenológicos $\quad 94$

4.1 Comparação entre os modelos fenomenológicos . . . . . . . . . . 95

4.1.1 A função de estrutura do nucleon . . . . . . . . . . . . . . 9 99

4.2 Produção de hadrons carregados . . . . . . . . . . . . . . 106

5 Física de saturação em processos $e A \quad \mathbf{1 1 2}$

5.1 Espalhamento elétron-núcleo . . . . . . . . . . . . . . 113

5.1 .1 A função peso nuclear inclusiva . . . . . . . . . . . . 113

5.1 .2 A função peso nuclear difrativa . . . . . . . . . . . . 116

5.1 .3 A função de estrutura nuclear inclusiva . . . . . . . . . . . 119

5.1.4 A função de estrutura nuclear difrativa . . . . . . . . . . . . 122

$5.1 .5 \quad$ A razão $\sigma_{d i f f} / \sigma_{t o t} \ldots \ldots \ldots \ldots \ldots \ldots$

6 Interação quarkonium-hádron a altas energias $\quad 134$

6.1 Introdução . . . . . . . . . . . . . . . . . . 134

$6.2 \mathrm{O}$ modelo . . . . . . . . . . . . . . . 136

6.2 .1 A Hamiltoniana de interação . . . . . . . . . . . . . . 136

$6.2 .2 \quad \mathrm{O}$ estado inicial $\ldots \ldots \ldots \ldots$. . . . . . . . . . . . . . . . . . .

6.2.3 O estado final . . . . . . . . . . . . . . 140

6.2.4 A interação com as fontes . . . . . . . . . . . . . 144

6.3 Resultados e Discussão . . . . . . . . . . . . . . . . . . 147

$\begin{array}{llr}7 & \text { Conclusão } & 159\end{array}$

$\begin{array}{ll}\text { Apêndice } & 161\end{array}$ 
Referências Bibliográficas 


\section{Lista de Figuras}

1.1 Espalhamento Inelástico Profundo. . . . . . . . . . . . . . . . . 11

1.2 Espalhamento Inelástico Profundo no modelo a partons. . . . . . . . . 17

1.3 Duas correções ao diagrama para o espalhamento inelástico profundo elétron-núcleon. . . . . . . . . . . . . . . . 18

1.4 Definção das quantidades empregadas. . . . . . . . . . . . . . 18

1.5 Representação em diagramas de Feynman das correções de $W_{\mu \mu^{\prime}}$ mostrado na figura $1.3 \ldots \ldots \ldots \ldots$

1.6 Função de estrutura $F_{2}$ (Extraído de [21]). . . . . . . . . . . . 29

1.7 Função de estrutura $F_{2}$ (Extraído de [21]) . . . . . . . . . . . 30

1.8 Distribuição de partons segundo a parametrização CTEQ5 [18]. ... 31

1.9 Resultados de ZEUS para a distribuição de glúons. Extraído . . . . . 36

1.10 Mapa da evolução da QCD. (Extraído de [19]) . . . . . . . . . . . 38

1.11 Resolução em termos da virtualidade $Q^{2}$. Fóton sondando o alvo. . . . 39

1.12 As funções de estrutura $F_{2}$ (Extraído de [50]) . . . . . . . . . . . 42

1.13 A razão $R_{C P}$ central para periférica de hádrons carregados e negativos como função de $p_{T}$ (Extraído de [25]) . . . . . . . . . . . . . 43

1.14 Função de estrutura difrativa (Extraído de [26]) . . . . . . . . . . . 45

1.15 Razão $\sigma^{D} / \sigma^{\text {tot }}$ medida pela Colaboração ZEUS (Extraído de [26]). . . . 46

2.1 Espalhamento Inelástico Profundo Difrativo. . . . . . . . . . . 60

2.2 Produção difrativa do par $q \bar{q} . \ldots \ldots \ldots \ldots$. . . . . . . . . . 62

2.3 Produção difrativa $q \bar{q} g \ldots \ldots \ldots \ldots$. . . . . . . . . . . . 68 
2.4 Contribuição do dipolo de cor efetivo. . . . . . . . . . . . . . 70

3.1 Diagrama para a emissão de um glúon na evolução de um dipolo e seu limite de $N_{c} \rightarrow \infty \ldots \ldots \ldots \ldots \ldots$

4.1 Escala de saturação para diferentes valores de $A$ e $x . \ldots . \ldots 95$

4.2 Dependência da amplitude de espalhamento de dipolo com o quadrado da separação do par $\left(r^{2}\right)$ para diferentes valores de $x \ldots \ldots$. . . . . 96

4.3 A amplitude de espalhamento de dipolo como uma função da variável

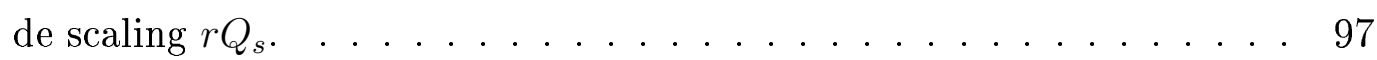

4.4 Dependência em energia da amplitude de espalhamento de dipolo para diferentes valores da separação quadrada do par $r^{2}=1 / Q^{2}$. . . . . 98

4.5 Comparação entre as previsões para $F_{2}\left(x, Q^{2}\right)$ em diferentes modelos e para diferentes valores de $Q^{2}$. Os dados são de ZEUS. . . . . . . . . 101

4.6 Comparação entre as previsões para $F_{2}\left(x, Q^{2}\right)$ em diferentes modelos e para diferentes valores de $Q^{2}$. Os dados são de ZEUS. . . . . . . . . . 102

4.7 Comparação entre as previsões para $F_{L}\left(x, Q^{2}\right)$ em diferentes modelos e para diferentes valores de $Q^{2} \ldots \ldots \ldots$. . . . . . . . . . . 103

4.8 Função de estrutura do próton para valores baixos de virtualidades do fóton. Os dados são de ZEUS. . . . . . . . . . . . . . . . . . . 104

4.9 Função de estrutura do próton para valores altos de virtualidades do fóton. Os dados são de ZEUS. . . . . . . . . . . . . . . . 105

4.10 Função de estrutura longitudinal do próton para valores baixos de virtualidades do fóton. Os dados são de ZEUS. . . . . . . . . . . . 106

4.11 Função de estrutura longitudinal do próton para valores altos de virtualidades do fóton. Os dados são de ZEUS. . . . . . . . . . . . . 107

4.12 Amplitude de espalhamento do dipolo em função da variável de scaling $r Q_{s} \ldots \ldots \ldots \ldots \ldots \ldots \ldots \ldots \ldots$

4.13 Amplitude de espalhamento do dipolo em função do tamanho do dipolo $r^{2} \ldots \ldots \ldots \ldots \ldots \ldots \ldots$ 
4.14 O comportamento em $Q^{2}$ da dimensão anômala efetiva. . . . . . . . . 110

$4.15 \mathrm{O}$ comportamento em $r Q_{s}$ da dimensão anômala efetiva. . . . . . . . 110

4.16 Comparação entre teoria e dados de BRAHMS para colisões minimumbias $d A u$ na energia do RHIC. . . . . . . . . . . . . . 111

5.1 A dependência em $\boldsymbol{r}$ da função peso fóton-núcleo para diferentes valores de número atômico $\left(x=10^{-5}\right.$ e $\left.Q^{2}=1 \mathrm{GeV}^{2}\right) \ldots \ldots \ldots \ldots$

5.2 A dependência em $\boldsymbol{r}$ da função peso fóton-núcleo para diferentes valores de número atômico $\left(x=10^{-5}\right.$ e $\left.Q^{2}=10 \mathrm{GeV}^{2}\right)$. . . . . . . . 116

5.3 A dependência em $\boldsymbol{r}$ da função peso fóton-núcleo para diferentes valores de número atômico, $x$ e $Q^{2} \ldots \ldots \ldots \ldots$. . . . . . . . . . . . . . .

5.4 A dependência em $\boldsymbol{r}$ da função peso difrativa fóton-núcleo (normalizada por $A^{2}$ ) em diferentes valores do número atômico e distintos modelos de saturação: IIM (painel à esquerda) e GBW (painel à direita). . . . . 118

5.5 A dependência em $\boldsymbol{r}$ da função peso difrativa fóton-núcleo (normalizada por $A^{2}$ ) em diferentes valores do número atômico e distintos modelos de saturação: IIM (painel à esquerda) e GBW (painel à direita). . . . . 118

5.6 Funções de estrutura nuclear como uma função de $x$ para diferentes valores de $A$ e $Q^{2} \ldots \ldots \ldots \ldots \ldots \ldots \ldots$

5.7 Razão entre as predições completa e linear para diferentes funções de estrutura nuclear. . . . . . . . . . . . . . . . . . . . . . 121

5.8 Razão entre as predições completa e linear para diferentes funções de estrutura nuclear. . . . . . . . . . . . . . . . . . 122

5.9 Razão entre as funções de estrutura nuclear como uma função de $x$ para diferentes valores de $A$ e fixo $Q^{2}=1$ e $10 \mathrm{GeV}^{2}$. . . . . . . . 123

5.10 Expoente efetivo como uma função de: (a) $Q^{2}$ and (b) $x$. . . . . . . . 124

5.11 Derivada logarítmica das funções de estrutura total e longitudinal com respeito à $Q^{2}$. 
5.12 Derivada logarítmica das funções de estrutura total e longitudinal com respeito à $Q^{2}$.

5.13 Derivada logarítmica das funções de estrutura total e longitudinal com respeito à $x$.

5.14 Derivada logarítmica das funções de estrutura total e longitudinal com respeito à $x$.

5.15 Função de estrutura difrativa $F_{2}^{D(3)}$ como função de $\beta$ e núcleos distintos. A componente $q \bar{q} g$ da função de estrutura difrativa é explicitamente mostrada.

5.16 Função de estrutura difrativa $F_{2}^{D(3)}$ como função de $\beta$ e núcleos distintos. As componentes transversal e $q \bar{q} g$ da função de estrutura difrativa são explicitamente mostrada. . . . . . . . . . . . . . . .

5.17 Predições para a funções de estrutura difrativa $x_{\mathbb{P}} F_{2}^{D(3)}\left(x_{\mathbb{P}}, \beta, Q^{2}\right)$ como função de $x_{\mathbb{P}}$ para diferentes valores de $\beta, Q^{2}$ e $A \ldots \ldots 130$

5.18 Predições para a funções de estrutura difrativa $x_{\mathbb{P}} F_{2}^{D(3)}\left(x_{\mathbb{P}}, \beta, Q^{2}\right)$ como função de $x_{\mathbb{P}}$ para diferentes valores de $\beta, Q^{2}$ e $A$, para dois modelos diferentes. . . . . . . . . . . . . . . . . . 130

5.19 A razão $R_{A 1, A 2}^{\text {diff }}\left(Q^{2}, \beta, x_{\mathbb{P}}\right)=\frac{F_{2, A 1}^{D(3)}\left(Q^{2}, \beta, x_{\mathbb{P}}\right)}{F_{2, A 2}^{D(3)}\left(Q^{2}, \beta, x_{\mathbb{P}}\right)}$ como função de $x_{\mathbb{P}}$ e $\beta$. Comparação entre as predições para a razão em diferentes valores de $A_{1}$. . 131

5.20 A razão $R_{A 1, A 2}^{d i f f}\left(Q^{2}, \beta, x_{\mathbb{P}}\right)=\frac{F_{2, A 1}^{D(3)}\left(Q^{2}, \beta, x_{\mathbb{P}}\right)}{F_{2, A 2}^{D(3)}\left(Q^{2}, \beta, x_{\mathbb{P}}\right)}$ como função de $x_{\mathbb{P}}$ e $\beta$. Comparação entre as previsões completa e linear. . . . . . . . . . . 131

5.21 A razão $R_{A 1, A 2}^{d i f f}\left(Q^{2}, \beta, x_{\mathbb{P}}\right)=\frac{F_{2, A 1}^{D(3)}\left(Q^{2}, \beta, x_{\mathbb{P}}\right)}{F_{2, A 2}^{D(3)}\left(Q^{2}, \beta, x_{\mathbb{P}}\right)}$ como função de $x_{\mathbb{P}}$ e $\beta$. Comparação entre as previsões completa e linear. . . . . . . . . . . . 132

5.22 A razão $R_{A 1, A 2}^{\text {diff }}\left(Q^{2}, \beta, x_{\mathbb{P}}\right)=\frac{F_{2, A 1}^{D(3)}\left(Q^{2}, \beta, x_{\mathbb{P}}\right)}{F_{2, A 2}^{D(3)}\left(Q^{2}, \beta, x_{\mathbb{P}}\right)}$ como função de $x_{\mathbb{P}}$ e $\beta$. Comparação entre as previsões completa e linear. . . . . . . . . . . . 132

5.23 A razão entre as seções de choque difrativa e total como função de $x$ e $W$ para diferentes valores de $A$ e $Q^{2}$. O limite de disco negro, $\sigma_{d i f f} / \sigma_{t o t}=1 / 2$, também é mostrado. . . . . . . . . . 
6.1 Interação Quarkonium-hádron no referencial de repouso do quarkonium. O hádron é um capacitor de cor que move-se para direita. O campo $\vec{E}^{a}$ está ao longo da direção $x$. Os pontos negros "gordos" representam as "placas" do capacitor: quark-antiquark para o píon e quark-diquark para o próton. . . . . . . . . . . . . . 138

6.2 Seção de choque $J / \psi+p$ com o modelo $A$ (linha pontilhada), modelo $B$ (linha tracejada) e a aproximação de Bhanot-Peskin (linha com estrelas). Curvas superiores: Campo $\vec{E}^{a}$ mais forte. Curvas inferiores: Campo $\vec{E}^{a}$ mais fraco. . . . . . . . . . . . . . . 149

6.3 O mesmo como na figura 1.2 para a seção de choque $\Upsilon+p . \ldots$. . . . 150

6.4 Seção de choque Píon-charmonium como uma função de $\sqrt{s}$ com vários modelos. . . . . . . . . . . . . . . . . 151

6.5 Seção de choque charmonium-hádron com o modelo $A$ (linha pontilhada) e com o modelo $B$ (linha tracejada). Curvas superiores: $\sigma_{J / \psi}+p$. Curvas inferiores $\sigma_{J / \psi}+\pi \ldots \ldots \ldots \ldots \ldots$

6.6 O mesmo como na figura 1.5, para a seção de choque bottonium-hádron. 153

6.7 Seção de choque charmonium-hádron como uma função do parâmentro de tamanho do charmonium para o modelo $A$ (linha pontilhada) e para o modelo $B$ (linha tracejada). . . . . . . . . . . . . . . 154

6.8 O mesmo como na figura 6.7, para a seção de choque bottonium-hádron. 155

6.9 Seção de choque charmonium-próton (linha tracejada) e charmoniumpíon (linha sólida) calculada com o modelo $C$ (interação de contato). 155

6.10 O mesmo como na figura 6.9, para a seção de choque bottonium-hádron. 156

6.11 Seção de choque charmonium-hádron para o modelo C, como uma fun-

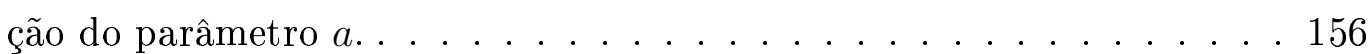

6.12 Seção de choque charmonium-hádron para o modelo C, como uma fun-

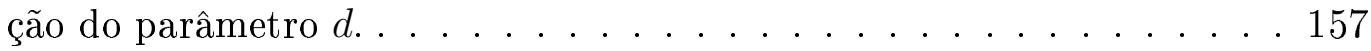

6.13 Seção de choque charmonium-hádron para o modelo C, como uma função de $\sqrt{s}$, para vários valores fixos do parâmetro $a$. . . . . . . 157 
6.14 Seção de choque charmonium-hádron para o modelo C, como uma função de $\sqrt{s}$, para vários valores fixos do parâmetro $d$. . . . . . . . 158 


\section{Introdução}

Nos próximos anos a física de interações fortes em altas energias terá entre seus objetivos principais a procura do condensado do vidro de cor (Color Glass Condensate ou CGC) e do plasma de quarks e gluons (Quark Gluon Plasma ou QGP). Eles podem ser considerados novos estados da matéria e existem evidências de que eles já tenham sido produzidos em laboratório. A confirmação experimental das observações iniciais poderá vir do Large Hadron Collider (LHC) nos próximos anos. O último capítulo desta tese trata de tema ligado à física do QGP e todos os outros tratam da física do CGC. No entanto, há uma ligação entre estas duas partes da tese, que está no fato de nos dois casos estudarmos colisões a altas energias entre um dipolo de cor e um alvo hadrônico. Além disso, nos dois casos o alvo hadrônico é um sistema com alta densidade (e grande número de ocupação) de gluons, o que justifica algum tipo de aproximação semi-clássica. Do ponto de vista cronológico, nós começamos fazendo física do QGP e terminamos fazendo física do CGC.

O texto que se segue descreve o resultado de quatro anos de trabalho que começou com o estudo da interação charmonium-hádron a altas energias, onde o charmonium é tratado como um dipolo ("pequeno") de cor e o hádron é visto como um capacitor ("grande") de cor. Os resultados deste trabalho foram publicados nas referências [1] e [2] e estão apresentados no capítulo 6 .

Um problema semelhante é o cálculo da seção de choque entre o charmonium híbrido ( $c-\bar{c}-$ gluon) e um hádron ordinário (méson ou núcleon), também a altas energias. Como parte deste projeto passamos a estudar a interação entre um glúon e o 
campo cromo-elétrico do alvo, que foi considerado um campo externo, como sugerido em [3]. Supondo que a energia fosse suficientemente alta e o alvo suficientemente denso, aproximamos o campo externo do alvo pela solução do modelo de McLerran e Venugopalan [4, 5]. Este estudo foi apresentado na reunião HADRONS/RANP-2004 [6] e faz parte dos "Proceedings" do encontro, mas não foi levado adiante. Deixamos o estudo das interações do charmonium para nos dedicarmos ao estudo da física do CGC, ou física da saturação. Esta mudança foi em parte motivada pelas discussões ocorridas na HADRONS/RANP e também pelo fato de que, em 2004 foi anunciado que o CGC tinha sido observado pela colaboração BRAHMS no RHIC. Desde então a busca por novas "assinaturas" deste novo estado da matéria tornou-se mais intensa.

E possível reescrever o espalhamento inelástico profundo (DIS) no formalismo de dipolos de cor, no qual o fóton se converte num par quark - antiquark e depois interage com o alvo. Neste formalismo, ao invés de extrair as distribuições de partons da análise de dados experimentais, o que fazemos é extrair a seção de choque de dipolo. Esta é a quantidade equivalente à distribuição de gluons que contém informações sobre a física não-perturbativa e também sobre o regime de saturação. Em princípio é possível calculá-las, pois elas são soluções de equações de evolução, análogas às equações de evolução das distribuições de partons. Muitos trabalhos já foram feitos sobre o assunto e solução já é conhecida em alguns limites. No entanto, este programa ainda não foi concluído e ainda é necessário usar parametrizações (modelos) para a seção de choque de dipolo. Passamos então a fazer testes de alguns dos modelos de seção de choque de dipolo. Uma das características a serem testadas é a universalidade. As distribuições de partons que constituem o núcleon são universais, ou seja, uma vez determinadas num processo serão as mesmas em todos os processos. Da mesma forma a seção de choque de dipolo, $\sigma_{d i p}$, deve ser universal. Assim a mesma $\sigma_{d i p}$ deve ser válida no espalhamento inelástico profundo e no espalhamento hádron-hádron (núcleo).

Começamos usando parametrizações obtidas em ajustes de dados de colisões deuteronouro no RHIC para reproduzir os dados do HERA. Verificamos que estes modelos não funcionavam bem. O cruzamento destas informações experimentais complementares 
mostrou que nenhum modelo é universal. Propusemos então uma nova parametrização que é capaz de descrever os dados do HERA e RHIC simultaneamente. Estes estudos geraram as publicações [7] e [8], cujo conteúdo está apresentado no capítulo 4.

Está em discussão o projeto de construir um acelerador elétron-ion onde hoje é o RHIC. O eRHIC, como ele é chamado, seria uma máquina dedicada ao DIS nuclear e por operar em altas energias (porém inferiores às do HERA) e com núcleos (que ampliam a densidade de partons do alvo) ele seria muito eficiente na busca dos efeitos do CGC. Em vista disso, usando um modelo de $\sigma_{\text {dip }}$ bem sucedido em DIS, fizemos sua extensão para o caso nuclear e calculamos vários observáveis, tais como as funções de estrutura nuclear inclusiva e difrativa e as seções de choque inclusiva e difrativa. Estes cálculos geraram as nossas publicações [9] e [10], cujo conteúdo está descrito no capítulo 5 .

Em resumo, todos os resultados originais estão contidos nos capítulos 4,5 e 6 . Nos três capítulos iniciais fizemos um esforço no sentido de organizar todo o material necessário para um leitor que não trabalhe nesta área possa entender de onde surgem as principais fórmulas usadas nos capítulos subsequentes. Este material foi coletado em livros texto, em teses de doutoramento e em artigos. Ele não é original mas está apresentado de uma forma diferente. Em alguns trechos há muitas passagens intermediárias, que certamente serão úteis a quem quiser reproduzir os resultados. Em outros trechos os cálculos estão apenas esboçados, em parte porque são muito longos e em parte porque não foi possível reproduzi-los completamente.

No capítulo 1, apresentamos os conceitos que serão utilizados no decorrer desta tese. Começamos com uma breve introdução a cromodinâmica quântica (QCD). Em seguida definimos as variáveis do espalhamento inelástico profundo e relacionamos a seção de choque fóton virtual-próton com a função de estrutura do próton $F_{2}$. $\mathrm{Na}$ sequência falamos do modelo a partons, que é equivalente ao diagrama de ordem zero da QCD. As correções a esse diagrama levam às equações de DGLAP. Uma delas é derivada explicitamente. Em seguida, tomando o limite de pequeno $x$, resolvemos a equação de evolução dos gluons, observando o rápido crescimento da população de 
gluons na região de pequeno $x$. Em seguida descrevemos qualitativamente o que é saturação e apresentamos algumas possíveis evidências experimentais deste fenômeno. Como os efeitos de saturação podem ser também importantes em processos difrativos, finalizamos o capítulo 1 fazendo algumas considerações gerais sobre difração.

No capítulo 2, apresentamos o formalismo de dipolos de cor e derivamos expressões para o espalhamento inelástico profundo inclusivo e difrativo na formulação de dipolos de cor. Partindo da fórmula de fatorização $k_{T}$ no espaço dos momento, reproduzimos as manipulações que nos levam a escrever a seção de choque fóton virtual-próton $\left(\sigma^{\gamma^{*} p}\right)$ como uma convolução da probabilidade do fóton virtual se converter num par quarkantiquark e a seção de choque de interação com o alvo. Essa interação é representada pela quantidade $\sigma_{d i p}$.

A dinâmica da quantidade $\sigma_{d i p}$ é estudada no capítulo 3 e no capítulo 7 apresentamos nossas conclusões.

Nesta tese optamos por deixar alguns termos e siglas em inglês, como, por exemplo, gauge, scaling, splitting, slope, CGC, QCD, etc. 


\section{Capítulo 1}

\section{Espalhamento inelástico profundo}

\subsection{A cromodinâmica quântica}

A Cromodinâmica Quântica (QCD) é a teoria de gauge que descreve as interações fortes [11]. A descrição é baseada num modelo de partículas elementares - os quarks - dotados de "carga de cor" e interagindo por troca de campos de gauge - os glúons. Trata-se de uma teoria quântica de campos, com simetria local de gauge $S U(3)$, correspondendo a três possíveis cores. A QCD é escrita de uma forma simples. Seus parâmetros são apenas as massas dos vários tipos (chamados "sabores") de quarks considerados e o valor da constante de acoplamento forte. A não ser pela simetria sob o grupo de gauge $S U(3)$ (ao invés do grupo $U(1)$ ), a forma da lagrangiana da QCD é a mesma que a da eletrodinâmica quântica (QED), com os quarks correspondendo aos elétrons e os glúons aos fótons. Os quarks e elétrons são férmions de spin 1/2 e os glúons e fótons são bósons vetoriais sem massa. Analogamente, a constante de acoplamento forte $\alpha_{s}$ corresponde à constante de estrutura fina $\alpha \approx 1 / 137$. O fato do grupo de gauge da QCD ser não-abeliano introduz, porém, diferenças qualitativas entre as duas teorias, refletindo as diferenças entre as interações fortes e as interações eletromagnéticas. Em particular, os glúons possuem carga de cor e portanto interagem entre si, ao contrário dos fótons. 
Uma característica importante da interação forte é que a constante de acoplamento $\alpha_{s}$ torna-se desprezível no limite de pequenas distâncias, ou equivalentemente no limite de altas energias ou momentos transferidos. Esta propriedade é chamada de liberdade assintótica. Aqui vale mencionar que o prêmio Nobel de Física de 2004 foi concedido a David Gross, David Politzer e Frank Wilczek, pela descoberta da liberdade assintótica na teoria das interações fortes.

A distâncias maiores (ou seja, a energias menores) há um aumento da intensidade da interação e acredita-se que a grandes distâncias a força de atração entre os quarks seja constante, determinando o confinamento de quarks e glúons dentro de hadrons. $\mathrm{O}$ fato de $\alpha_{s}$ não ser desprezível a baixas energias faz com que o estudo de fenômenos como o confinamento, o espectro de massa dos hadrons e a transição de desconfinamento a temperatura finita seja impossível com teoria de perturbação, que se baseia em uma expansão de acoplamentos fracos. Estes fenômenos devem ser estudados de maneira não perturbativa.

Nós nos limitaremos nesta tese ao uso da teoria de perturbação da QCD, uma vez que em espalhamentos a altas energias ela funciona muito bem.

\section{A Lagrangeana da QCD}

As regras de Feynman necessárias para uma análise perturbativa da QCD podem ser derivadas a partir da densidade de Lagrangeana $\mathcal{L}_{Q C D}=\mathcal{L}_{c l}+\mathcal{L}_{f c}+\mathcal{L}_{f t}$, sendo o termo clássico dado por:

$$
\mathcal{L}_{c l}=\sum_{\text {sabores }} \bar{q}_{a}\left(i \gamma^{\mu} D_{\mu}-m\right)_{a b} q_{b}-\frac{1}{4} F_{\mu \nu}^{A} F_{A}^{\mu \nu}
$$

onde

$$
\left(D_{\mu}\right)_{a b}=\partial_{\mu} \delta_{a b}+i g\left(t^{A} A_{\mu}^{A}\right)_{a b}
$$


é a derivada covariante, $q_{a}\left(\overline{q_{a}}\right)$ são os campos de quarks, $m$ é a massa do férmion e $g$ é a constante de acoplamento forte. A soma é feita sobre os sabores dos quarks: $u, d, s, c, b, t ; a, b=1,2,3$, são os índices de cor da representação fundamental associada aos quarks e $A=1,2, \ldots, 8$ são os índices de cor da representação adjunta associada aos glúons. O termo $\mathcal{L}_{c l}$ descreve a interação entre quarks de spin $1 / 2$ com massas $m$ e glúons de spin 1 com massas nulas. As matrizes gama satisfazem as relações de anti-comutação $\left\{\gamma^{\mu}, \gamma^{\nu}\right\}=2 g^{\mu \nu}$ e o tensor de campo $F_{\mu \nu}^{A}$ é dado por

$$
F_{\mu \nu}^{A}=\partial_{\mu} A_{\nu}^{A}-\partial_{\nu} A_{\mu}^{A}-g f^{A B C} A_{\mu}^{B} A_{\nu}^{C}
$$

As constantes de estrutura da $\mathrm{QCD}, f^{A B C}$ (antissimétricas sob a troca de índices) são definidas por

$$
\left[t^{A}, t^{B}\right]=i f^{A B C} t^{C}
$$

onde os t's são geradores do grupo $S U(3)$.

O segundo termo que aparece na densidade de Lagrangeana, $\mathcal{L}_{f c}$, surge devido à impossibilidade de se definir um propagador para o glúon sem uma escolha de gauge. Assim, as regras de Feynman para a propagação e interação de quarks e glúons só podem ser deduzidas após a introdução de um termo de fixação de gauge $\mathcal{L}_{f c}$. A escolha

$$
\mathcal{L}_{f c}=-\frac{1}{2 \lambda}\left(\partial^{\mu} A_{\mu}^{A}\right)^{2}
$$

determina a classe dos chamados "gauges covariantes". Porém, em uma teoria nãoabeliana como a $\mathrm{QCD}$, o termo de fixação de gauge $\mathcal{L}_{f c}$ deve vir acompanhado de um termo correspondente aos campos fantasmas de Fadeev-Popov, na forma

$$
\mathcal{L}_{f t}=\partial_{\mu} \eta^{A \dagger}\left(D_{A B}^{\mu} \eta^{B}\right)
$$

O campo $\eta^{A}$ é um campo escalar complexo que obedece a estatística fermiônica. Estes 
campos (fantasmas) cancelam os graus de liberdade não físicos que aparecem ao se trabalhar com gauges covariantes. A forma e mesmo a presença de fantasmas na Lagrangeana será dependente do gauge em que estivermos trabalhando. Se escolhermos o gauge de Lorentz, os fantasmas estarão presentes na Lagrangeana. Já para um gauge axial, esse termo não aparece na Lagrangeana.

\section{A constante de acoplamento $\alpha_{s}$}

Ao calcularmos diagramas de Feynman que contêm loops, encontramos divergências que necessitam ser regularizadas de tal forma que se tornem finitas. Estas divergências regularizadas podem ser posteriormente removidas, sendo absorvidas em redefinições de quantidades físicas através de um procedimento chamado de renormalização. Entretanto a subtração sistemática de divergências ultravioleta leva à introdução de uma escala de renormalização $\mu$ na teoria, fazendo com que quantidades renormalizadas, como, por exemplo, o acoplamento forte $\alpha_{s}$ dependam explicitamente de $\mu$. Uma vez que observáveis físicos devem ser independentes da escolha da escala $\mu$ (escala em que as subtrações das divergências ultravioletas são realizadas), uma mudança na escala de renormalização deve ser compensada por uma mudança na constante de acoplamento efetiva $\alpha_{s}$ no ponto de subtração $\mu$, definida como:

$$
\alpha_{s} \equiv \alpha_{s}\left(\mu^{2}\right)=\frac{g^{2}}{4 \pi}
$$

A forma como essa compensação é realizada pode ser estudada considerando-se um observável $R$ sem dimensões, envolvendo somente uma escala $Q^{2}$. O fato de $R$ não depender de $\mu$ implica que a derivada total com respeito a $\mu$ seja nula:

$$
\mu^{2} \frac{d}{d \mu^{2}} R\left(Q^{2} / \mu^{2}, \alpha_{s}\right)=\left[\mu^{2} \frac{\partial}{\partial \mu^{2}}+\mu^{2} \frac{\partial \alpha_{s}}{\partial \mu^{2}} \frac{\partial}{\partial \alpha_{s}}\right] R=0
$$


Definindo $t=\ln \frac{Q^{2}}{\mu^{2}}$ e a "função beta da QCD", $\beta\left(\alpha_{s}\right)$, como

$$
\beta\left(\alpha_{s}\right)=\mu^{2} \frac{\partial \alpha_{s}}{\partial \mu^{2}}
$$

a equação $(1,1.8)$ pode ser reescrita da seguinte forma

$$
\left[-\frac{\partial}{\partial t}+\beta\left(\alpha_{s}\right) \frac{\partial}{\partial \alpha_{s}}\right] R\left(e^{t}, \alpha_{s}\right)=0
$$

conhecida como "equação do grupo de renormalização", e determina como uma mudança no ponto de subtração $\mu$ é compensada por uma mudança no acoplamento $\alpha_{s}\left(\mu^{2}\right)$.

Introduzindo uma constante de acoplamento efetiva $\bar{\alpha}_{s}$, que é solução da equação

$$
\frac{d \bar{\alpha}_{s}\left(t, \alpha_{s}\right)}{d t}=\beta\left(\bar{\alpha}_{s}\right)
$$

$\operatorname{com} \alpha_{s}=\bar{\alpha}_{s}\left(t=0, \alpha_{s}\right)$, temos que

$$
t=\int_{\alpha_{s}}^{\bar{\alpha}_{s}\left(t, \alpha_{s}\right)} \frac{d \alpha}{\beta(\alpha)}
$$

Para determinarmos a evolução de $\alpha_{s}$ é necessário conhecer a função $\beta$. No nível de um loop a função $\beta$ é dada por [11]

$$
\beta\left(\alpha_{s}\right)=-b \alpha_{s}^{2} \quad \text { onde } \quad b=\frac{\left(11 C_{A}-4 n_{f} T_{R}\right)}{12 \pi}
$$

onde $C_{A}=3, T_{R}=1 / 2, n_{f}$ é o número de sabores leves e as correções de mais alta ordem foram desconsideradas. Substituindo esta expressão na equação $(1,1.12)$ obtemos

$$
\alpha_{s}\left(Q^{2}\right)=\frac{\alpha_{s}\left(\mu^{2}\right)}{1+\alpha_{s}\left(\mu^{2}\right) b t}
$$

Portanto, com o crescimento da escala $Q^{2}$ a constante de acoplamento diminui. Isto 
deve-se ao valor negativo da função $\beta$. A propriedade de a teoria tender a uma teoria livre para grandes escalas é denominada liberdade assintótica. Uma importante consequência desse comportamento da constante de acoplamento é que para pequenos valores de $Q^{2}$ o acoplamento torna-se grande e a teoria de perturbação deixa de ser válida, assim como as equações perturbativas acima. A equação $(1,1.14)$ pode ser reescrita como

$$
\alpha_{s}\left(Q^{2}\right)=\frac{1}{b \ln \left(Q^{2} / \Lambda_{Q C D}^{2}\right)}
$$

onde $\Lambda_{Q C D}$ é um parâmetro determinado pelos dados experimentais.

\subsection{O espalhamento inelástico profundo}

O espalhamento inelástico profundo (DIS) é capaz de fornecer um importante teste para a cromodinâmica quântica perturbativa (pQCD). Além disso ele é o modo mais direto de explorar a estrutura interna dos hádrons. Vamos descrever a cinemática do processo de espalhamento lépton-hádron.

Considere o processo no qual um feixe de léptons (elétron, múon ou neutrino) bombardeia um alvo hadrônico (geralmente um núcleon $N$ ) e sofre espalhamento inlelástico

$$
l+N \rightarrow l+X,
$$

onde $l$ representa o lépton, $N$ o núcleon e $X$ o sistema de hádrons produzidos através de processos inelásticos. Experimentalmente não identificamos os hádrons produzidos $X$ e medimos apenas o momento do lépton no estado final. Experimentos deste tipo são chamados de experimentos inclusivos. Quando conseguimos identificar todos os os hádrons produzidos no estado final temos os chamados experimentos exclusivos. $\mathrm{O}$ diagrama de Feynman para o processo descrito em (1,2.16) está representado na figura 1.1 .

Para estudarmos os experimentos inclusivos consideramos três variáveis cinemáticas 


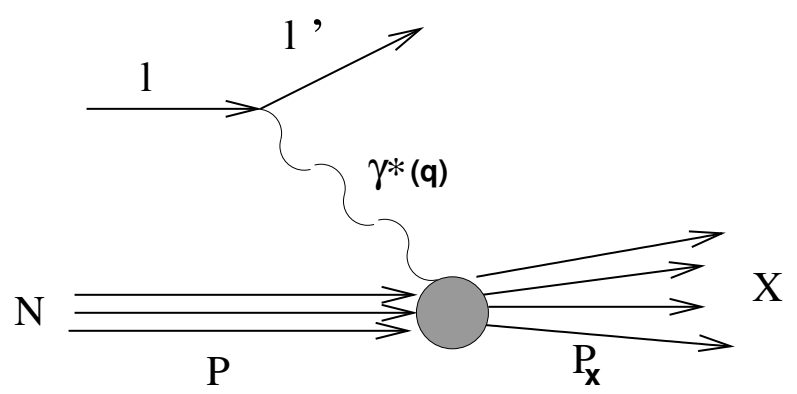

Figura 1.1: Espalhamento Inelástico Profundo.

independentes:

$$
s=(l+P)^{2}, \quad q^{2} \equiv-Q^{2}=\left(l-l^{\prime}\right)^{2}, \quad W^{2}=(P+q)^{2},
$$

onde $l$ e $l^{\prime}$ são os quadri-momentos inicial e final do lépton respectivamente; $P$ é o quadri-momento do núcleon e $q^{2}$ é o quadri-momento quadrado transferido do lépton. $Q^{2}$ será interpretado como a virtualidade do fóton. A variável $s$ corresponde ao quadrado da energia total do sistema lépton-núcleon no referencial do centro de massa e $W^{2}$ é o quadrado da massa invariante do estado hadrônico final $X$. No referencial de repouso do alvo (referencial do laboratório) $P=\left(m_{N}, 0,0,0\right)$, onde $m_{N}$ é a massa do núcleon, e as variáveis $s, q^{2}$ e $W^{2}$ são reescritas da seguinte forma:

$$
\begin{aligned}
s & =m_{N}\left(2 E+m_{N}\right), \\
q^{2} & =-4 E^{\prime} \operatorname{sen}^{2}(\theta / 2), \\
W^{2} & =m_{N}^{2}+2 m_{N}\left(E-E^{\prime}\right)+q^{2},
\end{aligned}
$$

onde a massa do lépton foi desprezada e o ângulo $\theta$ é o ângulo de espalhamento do lépton no referencial do laboratório. Aqui podemos identificar a energia transferida $\nu \operatorname{com} \nu=\left(E-E^{\prime}\right)$. Frequentemente define-se a variável $\nu$ como: $\nu=\frac{P \cdot q}{m_{N}}$. Outras 
variáveis que surgem no DIS são:

$$
\begin{aligned}
& x=\frac{Q^{2}}{2 P . q}=\frac{Q^{2}}{2 m_{N} \nu}=\frac{Q^{2}}{W^{2}+Q^{2}-m_{N}^{2}}, \\
& y=\frac{P . q}{P . l}=\frac{W^{2}+Q^{2}-m_{N}^{2}}{s-m_{N}^{2}}
\end{aligned}
$$

onde $x$ é identificada como a variável de Bjorken que será bastante utilizada no decorrer dessa tese, especialmente quando considerarmos a região em que essa variável é muito pequena; e y representa a fração de energia do lépton inicial carregada pelo fóton trocado, $y=\nu / E$. Em outras palavras, $y$ é a fração da energia do lépton inicial transferida ao sistema hadrônico. Uma relação útil entre $x, y$ e $Q^{2}$ é dada por:

$$
x y=\frac{Q^{2}}{s-m_{N}^{2}} \approx \frac{Q^{2}}{s} .
$$

Como $W^{2} \geq m_{N}^{2}$, a variável de Bjorken $(x)$ e $y$ assumem valores entre $0<x, y<1$. O nome "deep inelastic" vem do regime cinemático onde ambos $m_{N} \nu$ e $Q^{2}$ são muito maiores do que $m_{N}^{2}$, com $x$ fixo e finito. A seção de choque do processo descrito acima é:

$$
d \sigma=\frac{1}{4(l . P)} \frac{1}{2} \sum_{s_{l}, s_{l^{\prime}}} \frac{1}{2} \sum_{S} \sum_{X} \int \frac{d^{3} P_{X}}{(2 \pi)^{3} 2 P_{X}^{0}}(2 \pi)^{4} \delta^{4}\left(P+l-P_{X}-l^{\prime}\right)|M|^{2} \frac{d^{3} l^{\prime}}{(2 \pi)^{3} 2 E^{\prime}}
$$

onde o quadrado da amplitude é dado por:

$$
|M|^{2}=\frac{e^{4}}{q^{4}}\left[\bar{u}_{l^{\prime}}\left(l^{\prime}, s_{l^{\prime}}\right) \gamma_{\mu} u_{l}\left(l, s_{l}\right)\right]^{*}\left[\bar{u}_{l^{\prime}}\left(l^{\prime}, s_{l^{\prime}}\right) \gamma_{\nu} u_{l}\left(l, s_{l}\right)\right]\left\langle X\left|J^{\mu}(0)\right| P, S\right\rangle^{*}\left\langle X\left|J^{\nu}(0)\right| P, S\right\rangle
$$

Nesse ponto podemos introduzir o tensor hadrônico $W^{\mu \nu}$ :

$$
\begin{aligned}
W^{\mu \nu} & \equiv \frac{1}{2 \pi} \frac{1}{2} \sum_{S} \sum_{X} \int \frac{d^{3} P_{X}}{(2 \pi)^{3} 2 P_{X}^{0}}(2 \pi)^{4} \delta^{4}\left(P+q-P_{X}\right)\left\langle P, S\left|J^{\mu}(0)\right| X\right\rangle\left\langle X\left|J^{\nu}(0)\right| P, S\right\rangle \\
& =\frac{1}{2 \pi} \int d^{4} z e^{i q z}\left\langle N\left|J^{\mu}(z) J^{\nu}(0)\right| N\right\rangle
\end{aligned}
$$


onde $J_{\mu}$ é a corrente de transição hadrônica; e o tensor leptônico $L_{\mu \nu}$ :

$$
\begin{aligned}
L_{\mu \nu} & \equiv \frac{1}{2} \sum_{s_{l}, s_{l^{\prime}}}\left[\bar{u}_{l^{\prime}}\left(l^{\prime}, s_{l^{\prime}}\right) \gamma_{\mu} u_{l}\left(l, s_{l}\right)\right]^{*}\left[\bar{u}_{l^{\prime}}\left(l^{\prime}, s_{l^{\prime}}\right) \gamma_{\nu} u_{l}\left(l, s_{l}\right)\right] \\
& =\frac{1}{2} \operatorname{Tr}\left[\not \gamma_{\mu} l^{\prime} \gamma_{\nu}\right] \\
& =2\left(l_{\mu} l_{\nu}^{\prime}+l_{\nu} l_{\mu}^{\prime}-g_{\mu \nu} l . l^{\prime}\right)
\end{aligned}
$$

Substituindo $(1,2.23)$ e $(1,2.24)$ em $(1,2.21)$ obtemos:

$$
\frac{d^{2} \sigma}{d E^{\prime} d \Omega}=\frac{\alpha_{e m}^{2}}{2 m_{N} Q^{4}} \frac{E^{\prime}}{E} L_{\mu \nu} W^{\mu \nu}
$$

onde $d \Omega$ é o ângulo sólido que identifica a direção do lépton que sai (l'):

$$
d \Omega=d \cos \theta d \phi \rightarrow \Omega \equiv(\theta, \phi)
$$

$\mathrm{e}$

$$
\alpha_{e m}=\frac{e^{2}}{4 \pi}
$$

O tensor hadrônico é construído a partir de $g^{\mu \nu}, p^{\mu}$ e $q^{\mu}$ presentes no vértice hadrônico

$$
W^{\mu \nu}=-W_{1} g^{\mu \nu}+\frac{W_{2}}{m_{N}^{2}} p^{\mu} p^{\nu}+\frac{W_{4}}{m_{N}^{2}} q^{\mu} q^{\nu}+\frac{W_{5}}{m_{N}^{2}}\left(p^{\mu} q^{\nu}+q^{\mu} p^{\nu}\right)
$$

onde $W_{1}, W_{2}, W_{4} W_{5}$ são as funções de estrutura inelásticas. A partir da conservação de corrente no vértice hadrônico:

$$
q^{\mu} W^{\mu \nu}=0
$$

obtemos:

$$
W^{\mu \nu}=W_{1}\left[-g^{\mu \nu}+\frac{q^{\mu} q^{\nu}}{q^{2}}\right]+\frac{W_{2}}{m_{N}^{2}}\left(p^{\mu}-\frac{p \cdot q}{q^{2}} q^{\mu}\right)\left(p^{\nu}-\frac{p \cdot q}{q^{2}} q^{\nu}\right) .
$$

Assim escrevemos o produto $L_{\mu \nu} W^{\mu \nu}$, que aparece na equação $(1,2.25)$, no referencial 
do laboratório como:

$$
L_{\mu \nu} W^{\mu \nu}=4 E E^{\prime}\left[2 W_{1} \operatorname{sen}^{2} \frac{\theta}{2}+W_{2} \cos ^{2} \frac{\theta}{2}\right]
$$

Substituindo $(1,2.28)$ na expressão $(1,2.25)$ chegamos em:

$$
\frac{d^{2} \sigma}{d E^{\prime} d \Omega}=\frac{4 \alpha_{e m}^{2} E^{\prime 2}}{Q^{4}}\left[2 W_{1} \operatorname{sen}^{2} \frac{\theta}{2}+W_{2} \cos ^{2} \frac{\theta}{2}\right]
$$

A seção de choque diferencial pode ser reescrita em termos das variáveis do DIS

$$
\begin{aligned}
\left.\frac{d \sigma}{d y d x}\right|_{l a b}= & \frac{2 \pi m_{N} E y}{E^{\prime}} \frac{\alpha_{e m}^{2}}{4 E^{2}\left(\frac{x y m_{N}}{2 E^{\prime}}\right)^{2}} \\
& \times\left[2 W_{1} \frac{x y m_{N}}{2 E^{\prime}}+W_{2} \frac{E}{E^{\prime}}\left(1-y-\frac{m_{N} x y}{2 E}\right)\right] \\
= & \frac{2 \pi \alpha_{e m}^{2}}{E m_{N} x^{2} y}\left[x y m_{N} W_{1}+E\left(1-y-\frac{m_{N} x y}{2 E}\right) W_{2}\right] \\
= & \frac{2 \pi \alpha_{e m}^{2}}{E m_{N} x^{2} y^{2}}\left[x y^{2} F_{1}+\left(1-y-\frac{m_{N} x y}{2 E}\right) F_{2}\right]
\end{aligned}
$$

onde definimos as funções de estrutura adimensionais:

$$
F_{1}\left(x, Q^{2}\right) \equiv m_{N} W_{1}\left(\nu, Q^{2}\right)
$$

$\mathrm{e}$

$$
F_{2}\left(x, Q^{2}\right) \equiv \nu W_{2}\left(\nu, Q^{2}\right)
$$

Lembrando que $y=\frac{Q^{2}}{2 m_{N} E x}$, podemos escrever

$$
d y=\frac{d Q^{2}}{2 m_{N} x E}
$$

e

$$
x^{2}=\frac{Q^{4}}{4 m_{N}^{2} E^{2} y^{2}} .
$$


Portanto,

$$
\left.\frac{d \sigma}{d x d Q^{2}}\right|_{l a b}=\frac{4 \pi \alpha_{e m}^{2}}{x Q^{4}}\left[x y^{2} F_{1}+\left(1-y-\frac{x y m_{N}}{2 E}\right) F_{2}\right]
$$

Quando $\sqrt{s} \gg m_{N}$ :

$$
\left.\frac{d \sigma}{d x d Q^{2}}\right|_{l a b} \approx \frac{4 \pi \alpha_{e m}^{2}}{x Q^{4}}\left[x y^{2} F_{1}+(1-y) F_{2}\right]
$$

As funções de estrutura descrevem a estrutura do próton (e/ou núcleon) medida no espalhamento inelástico profundo inclusivo.

As funções de estrutura do DIS podem ser relacionadas [12] com a seção de choque total de absorção do fóton virtual:

$$
\sigma_{\lambda}^{\gamma^{*} N}\left(x, Q^{2}\right)=\frac{2 \pi^{2} \alpha_{e m}}{m_{N} \sqrt{\nu^{2}+Q^{2}}} \varepsilon_{\mu}^{(\lambda)} \varepsilon_{\nu}^{(\lambda)^{*}} W^{\mu \nu}
$$

onde $\varepsilon_{\mu}^{(\lambda)}$ é a polarização quadri-vetorial de um fóton virtual de helicidade $\lambda$. Em termos de $F_{1}$ e $F_{2}$ as seções de choque transversal e longitudinal são dadas por:

$$
\sigma_{L}^{\gamma^{*} N}=\frac{4 \pi^{2} \alpha_{e m}}{Q^{2}}\left(F_{2}-2 x F_{1}\right)
$$

$\mathrm{e}$

$$
\sigma_{T}^{\gamma^{*} N}=\frac{4 \pi^{2} \alpha_{e m}}{Q^{2}} 2 x F_{1} .
$$

E agora definindo:

$$
F_{T}=2 x F_{1}
$$

$\mathrm{e}$

$$
F_{L}=F_{2}-2 x F_{1}
$$

temos:

$$
\sigma_{L, T}^{\gamma^{*} N}=\frac{4 \pi^{2} \alpha_{e m}}{Q^{2}} F_{L, T}\left(x, Q^{2}\right) .
$$


Observe que: $F_{L}+F_{T}=F_{2}$. Finalmente relacionamos a seção de choque total com $F_{2}$ da seguinte forma:

$$
\sigma^{\gamma^{*} N}=\frac{4 \pi^{2} \alpha_{e m}}{Q^{2}} F_{2}\left(x, Q^{2}\right)
$$

\subsection{O modelo a pártons}

Em 1969 Bjorken mostrou que se o núcleon fosse feito de férmions puntiformes e não interagentes, quando: $\nu, Q^{2} \rightarrow \infty$ e mantendo $x=\frac{Q^{2}}{2 m_{N} \nu}$ fixo, $F_{1}$ e $F_{2}$ deveriam ser funções apenas de uma variável, $x$ e não mais de duas. Assim as funções de estrutura deveriam exibir o chamado scaling de Bjorken [13]:

$$
F_{i}\left(x, Q^{2}\right) \approx F_{i}(x), \quad i=1,2
$$

Como veremos, essa relação é válida apenas no modelo a pártons não interagentes, mas em QCD existe uma dependência suave em $Q^{2}$, de tipo logarítmica, e essa relação é violada. Esta é a violação do scaling de Bjorken.

Ainda em 1969 dados experimentais do SLAC confirmaram as previsões de Bjorken, mostrando que as funções $F_{1}$ e $F_{2}$ dependiam realmente da razão $Q^{2} / \nu$, e não de cada uma delas separadamente. Esse resultado foi interpretado [14, 15] como uma evidência de que o próton é composto por partículas elementares (puntiformes): os pártons. Nesta interpretação o fóton virtual $\left(\gamma^{*}\right)$ espalha-se incoerentemente com os constituintes internos do núcleon.

A hipótese de espalhamento incoerente implica que a seção de choque total $\gamma^{*}$ próton é igual à soma das seções de choque $\gamma^{*}$-párton, pesada pela probabilidade $f_{i}(\xi)$ de encontrar um párton $q_{i}$ dentro do próton carregando uma fração $\xi$ de seu quadri-momento:

$$
\frac{d^{2} \sigma}{d x d Q^{2}}=\sum_{i} \int_{0}^{1} d \xi f_{i}(\xi) \frac{d^{2} \hat{\sigma}}{d x d Q^{2}}
$$

Deste modo introduzimos a distribuição de partons do tipo $i, f_{i}(\xi)$, dentro do próton. A expressão $(1,3.39)$ reflete a fatorização da seção de choque do DIS em uma interação 


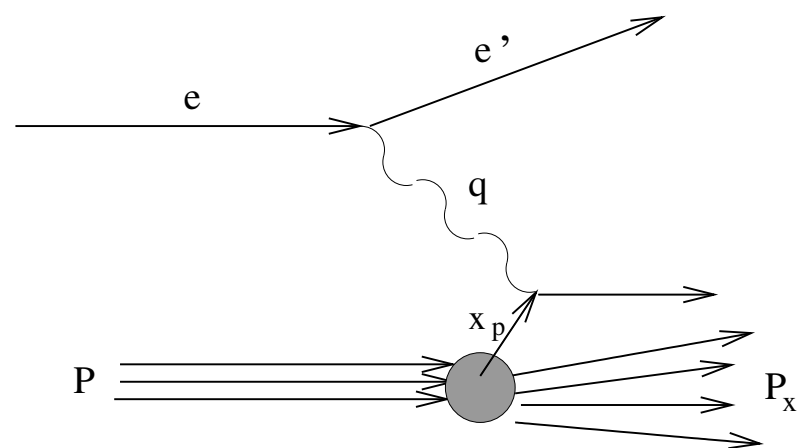

Figura 1.2: Espalhamento Inelástico Profundo no modelo a partons.

de curta distância, descrita pela seção de choque partônica $d \hat{\sigma}(\xi)$, e uma função que contém os efeitos de longa distância, a distribuição de partons $f(\xi)$.

Assumindo que os partons sejam férmions de Dirac com spin 1/2, carregando uma fração $\xi$ do momento do próton, o seguinte resultado é encontrado no modelo a partons [16]:

$$
F_{2}(x)=2 x F_{1}(x)=\sum_{i} e_{i}^{2} \int_{0}^{1} d \xi \delta(x-\xi) \xi f_{i}(\xi)=\sum_{i} e_{i}^{2} x f_{i}(x)
$$

onde $e_{i}$ é a carga elétrica do quark de sabor $i$. A relação de Callan-Gross, $F_{2}(x)=$ $2 x F_{1}(x)$ (a seção de choque longitudinal é zero!), vêm da hipótese de spin igual a 1/2. Além disso, o $x$ de Bjorken é igual à fração de momento do parton que colide. Vemos assim que a função de estrutura $F_{2}(x)$ mede a distribuição de partons dentro do próton.

\subsection{As equações de DGLAP}

As equações de Dokshitzer-Gribov-Lipatov-Altarelli-Parisi (DGLAP) descrevem a influência das correções perturbativas da QCD nas funções de distribuição que entram no modelo de partons dos processos de espalhamento inelástico profundo. Vamos analisar aqui apenas as correções provenientes dos gráficos na figura 1.3 [17].

O processo a ser estudado é o $\gamma q \rightarrow g q$ como mostrado na figura 1.4. 


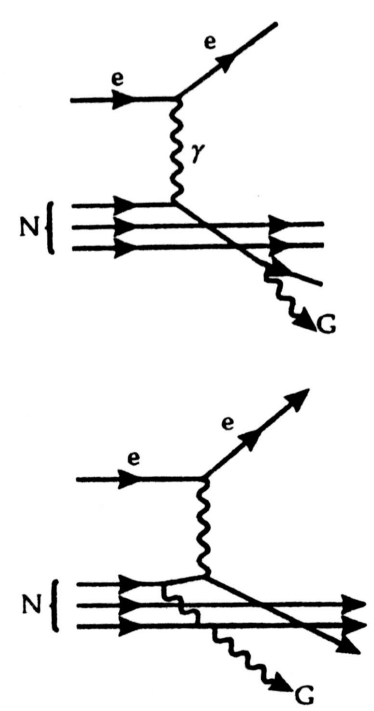

Figura 1.3: Duas correções ao diagrama para o espalhamento inelástico profundo elétron-núcleon.
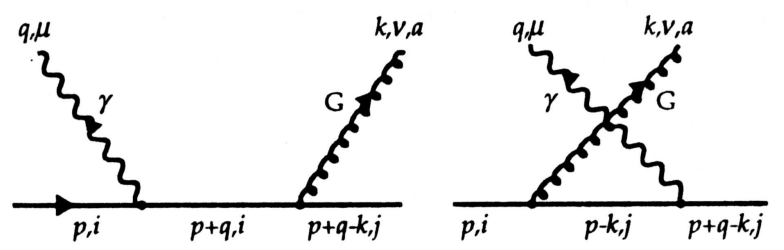

Figura 1.4: Definção das quantidades empregadas.

Queremos calcular a contribuição desses gráficos ao tensor hadrônico $W_{\mu \nu}$, que é dada pelo quadrado dessas amplitudes. Vamos calcular a parte imaginária dos diagramas apresentados na figura 1.5. A parte imaginária desses diagramas nos fornece a contribuição on-shell das linhas intermediárias, que nada mais é do que o quadrado das amplitudes que queremos calcular. A parte imaginária de qualquer amplitude pode ser obtida usando-se as regras de Cutkosky, ou seja, substituindo-se os denominadores dos propagadores das linhas intermediárias por:

$$
\begin{gathered}
\frac{1}{k^{2}+i \varepsilon} \rightarrow-2 i \pi \delta\left(k^{2}\right) \theta\left(k_{0}\right) \\
\frac{1}{(p+q-k)^{2}+i \varepsilon} \rightarrow-2 i \pi \delta\left[(p+q-k)^{2}\right] \theta\left(p_{0}+q_{0}-k_{0}\right)
\end{gathered}
$$



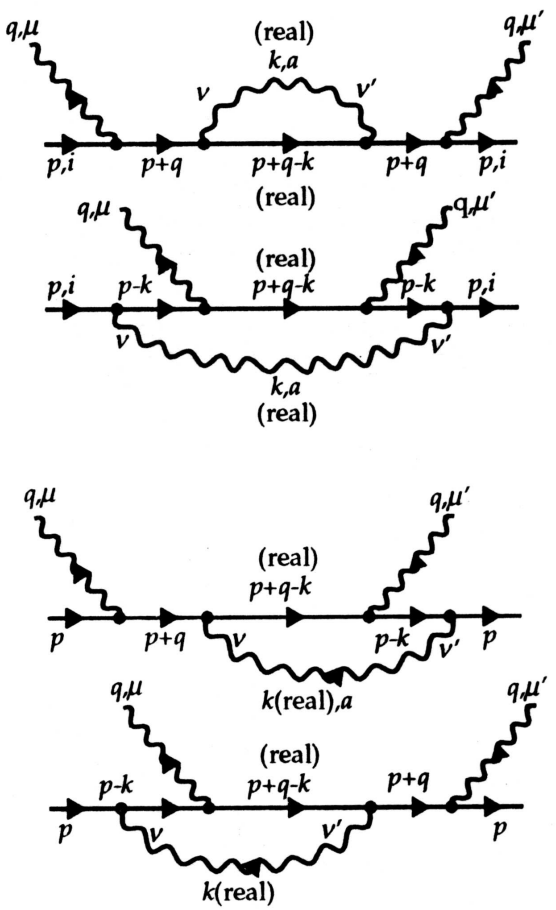

Figura 1.5: Representação em diagramas de Feynman das correções de $W_{\mu \mu^{\prime}}$ mostrado na figura 1.3.

Assim, trabalhando no gauge de Feynman, $\alpha=1$, obtemos

$$
\begin{gathered}
W_{\mu \mu^{\prime}}=\frac{1}{2 \pi} \int \frac{d^{4} k}{(2 \pi)^{4}} \frac{1}{2} \sum_{s} \bar{u}(p, s) i Q_{f} i g \frac{\lambda_{j i}^{a}}{2}\left[\gamma_{\mu^{\prime}} i S(p+q) \gamma_{\rho}\right. \\
\left.+\gamma_{\rho} i S(p-k) \gamma_{\mu^{\prime}}\right]\left(-i g^{\rho \nu}\right)\left(-2 i \pi \delta\left(k^{2}\right) \theta\left(k_{0}\right)\right) i(\not p+\not-\not p) \\
\left(-2 i \pi \delta\left[(p+q-k)^{2}\right] \theta\left(p_{0}+q_{0}-k_{0}\right)\right) i g \frac{\lambda_{i j}^{a}}{2} i Q_{f}\left[\gamma_{\nu} i S(p+q) \gamma_{\mu}\right. \\
\left.+\gamma_{\mu} i S(p-k) \gamma_{\nu}\right] u(p, s),
\end{gathered}
$$

onde $\lambda^{a}$ são as matrizes de Gell-Mann e $Q_{f}$ é a carga elétrica do quark de sabor $f$. Fazemos agora a média sobre as cores

$$
\frac{1}{3} \sum_{a} \frac{\lambda_{j i}^{a}}{2} \frac{\lambda_{i j}^{a}}{2}=\frac{1}{3} \sum_{a} \operatorname{Tr}\left[\frac{\lambda^{a}}{2}\right]^{2}=\frac{1}{3} \sum_{a} \frac{\delta_{a a}}{2}=\frac{4}{3}
$$


e assim

$$
\begin{gathered}
W_{\mu \mu^{\prime}}=\frac{1}{2 \pi}(2 \pi)^{2} \frac{4}{3} Q_{f}^{2} g^{2} \frac{1}{2} \sum_{s} \int \frac{d^{4} k}{(2 \pi)^{4}} \bar{u}_{i}(p, s)\left\{\left[\gamma_{\mu^{\prime}} \frac{\not p+q}{(p+q)^{2}} \gamma_{\rho}\right.\right. \\
\left.\left.+\gamma_{\rho} \frac{\not p-\not \not d}{(p-k)^{2}} \gamma_{\mu^{\prime}}\right](\not p+\not d-\not \supset)\left[\gamma^{\rho} \frac{\not p+\not d}{(p+q)^{2}} \gamma_{\mu}+\gamma_{\mu} \frac{\not p-\not p}{(p-k)^{2}} \gamma^{\rho}\right]\right\}_{i j} \\
u_{j}(p, s) \delta\left(k^{2}\right) \theta\left(k_{0}\right) \delta\left[(p+q-k)^{2}\right] \theta\left(p_{0}+q_{0}-k_{0}\right) .
\end{gathered}
$$

Lembrando que $\sum_{s}=\bar{u}_{i}(p, s) u_{j}(p, s)=\not p_{j i}$ e fazendo também a média sobre as polarizações dos fótons dada por:

$$
W_{\mu \mu^{\prime}} \rightarrow \frac{W_{\mu \mu^{\prime}}}{2}
$$

temos

$$
\begin{aligned}
W_{\mu \mu^{\prime}}= & \frac{4}{3} Q_{f}^{2} g^{2} \frac{1}{(2 \pi)^{3}} \frac{1}{4} \int d^{4} k S_{\mu \mu^{\prime}} \delta\left(k^{2}\right) \theta\left(k_{0}\right) \times \\
& \delta\left[(p+q-k)^{2}\right] \theta\left(p_{0}+q_{0}-k_{0}\right)
\end{aligned}
$$

onde

$$
\begin{aligned}
& S_{\mu \mu^{\prime}}=\operatorname{Tr}\left[\not p\left(\gamma_{\mu^{\prime}} \frac{\not p+q d}{(p+q)^{2}} \gamma_{\rho}+\gamma_{\rho} \frac{\not p-\not p}{(p-k)^{2}} \gamma_{\mu^{\prime}}\right)(\not p+\not d-\not \not)\right. \\
& \left.\left(\gamma^{\rho} \frac{\not p+\not d}{(p+q)^{2}} \gamma_{\mu}+\gamma_{\mu} \frac{\not p-\not p}{(p-k)^{2}} \gamma^{\rho}\right)\right]
\end{aligned}
$$

Como já sabemos que toda informação fornecida por $W_{\mu \mu^{\prime}}$ está contida nas funções de estrutura $W_{1}$ e $W_{2}$, basta calcular $W_{\mu}^{\mu}$ e $p^{\mu} p^{\mu^{\prime}} W_{\mu \mu^{\prime}}$. Para isso vamos definir as variáveis de Mandelstam:

$$
\begin{aligned}
t & =(p-k)^{2}=-2 p \cdot k, \quad\left(p^{2}=k^{2}=0\right) \\
s & =(q+p)^{2}=q^{2}+2 p \cdot q=2 \nu-Q^{2} \\
u & =(q-k)^{2}=(q+p-k-p)^{2}=(q+p-k)^{2} \\
& -2 p \cdot(q+p-k)=-2 \nu-t
\end{aligned}
$$


onde $\nu=p . q$ e $Q^{2}=-q^{2}$. Podemos também escrever $s=(p+q-k+k)^{2}=2 k \cdot(p+q-k)$

$$
s=2 k \cdot(p+q)
$$

já que devido à função delta, $(q+p-k)^{2}=0$. Reescrevendo $S_{\mu \mu^{\prime}}$ em termos das variáveis de Mandelstam temos

$$
\begin{aligned}
& S_{\mu}^{\mu}=\operatorname{Tr}\left[\not p\left(\gamma_{\mu} \frac{\not p+\not q}{s} \gamma_{\rho}+\gamma_{\rho} \frac{\not p-\not p}{t} \gamma_{\mu}\right)(\not p+\not d-\not \supset)\right. \\
&\left.\left(\gamma^{\rho} \frac{\not p+q q}{s} \gamma^{\mu}+\gamma^{\mu} \frac{\not p-\not p}{t} \gamma^{\rho}\right)\right] .
\end{aligned}
$$

Finalmente podemos escrever

$$
S_{\mu}^{\mu}=-8\left(\frac{t}{s}+\frac{s}{t}-\frac{2 Q^{2} u}{s t}\right)
$$

Usando a equação( $1,4.42)$ podemos calcular $p^{\mu} p^{\mu^{\prime}} S_{\mu \mu^{\prime}}$ :

$$
\begin{aligned}
& p^{\mu} p^{\mu^{\prime}} S_{\mu \mu^{\prime}}=\operatorname{Tr}\left[\not p\left(\not p \frac{\not p+\not d}{s} \gamma_{\rho}+\gamma_{\rho} \frac{\not p-\not p}{t} \not p\right)(\not p+\not d-\not p)\right. \\
& \left.\left(\gamma^{\rho} \frac{\not p+\not d}{s} \not p+\not p \frac{\not p-\not \not p}{t} \gamma^{\rho}\right)\right]=\operatorname{Tr}\left[\gamma^{\rho} \not \not \gamma \gamma_{\rho} \frac{\not p-\not \not p}{t} \not p(\not p+\not d\right.
\end{aligned}
$$

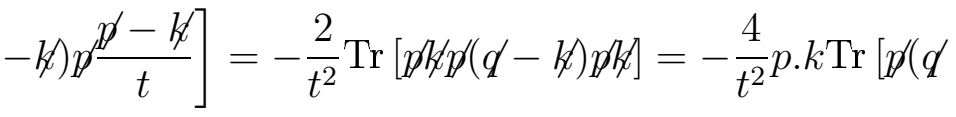

$$
\begin{aligned}
& -\not k) \not p l d]=-\frac{8}{t^{2}}(p \cdot k)^{2} \operatorname{Tr}[(\not-\not k) \not p]=-\frac{8}{t^{2}} \frac{t^{2}}{4} 4(q-k) \cdot p \\
& =-8\left[p \cdot(q-k)+p^{2}\right]=-8 p \cdot(q-k+p)=-8\left(-\frac{u}{2}\right)
\end{aligned}
$$

ou seja,

$$
p^{\mu} p^{\mu^{\prime}} S_{\mu \mu^{\prime}}=4 u=-4(2 \nu+t) .
$$

Assim, para calcular $W_{\mu \mu^{\prime}}$ temos que integrar funções de $t, \nu$ e $Q^{2}$. Considerando a 
integral na equação $(1,4.41)$ :

$$
I=\int d^{4} k f\left(t, \nu, Q^{2}\right) \delta\left(k^{2}\right) \delta\left[(p+q-k)^{2}\right] \theta\left(k_{0}\right) \theta\left(p_{0}+q_{0}-k_{0}\right)
$$

Depois das integrações obtemos:

$$
\begin{aligned}
I & =-\pi \int_{0}^{-2 \nu} d t \frac{1}{2 p} \frac{1}{2 \sqrt{Q^{2}}} f\left(t, \nu, Q^{2}\right) \theta(p-|\vec{k}|) \\
& =\frac{\pi}{4 \nu} \int_{-2 \nu}^{0} d t f\left(t, \nu, Q^{2}\right) .
\end{aligned}
$$

Finalmente, usando as equações $(1,4.41)$ e $(1,4.47)$ podemos escrever

$$
W_{\mu \mu^{\prime}}=\frac{4}{3} g^{2} \frac{Q_{f}^{2}}{(2 \pi)^{3}} \frac{1}{4} \frac{\pi}{4 \nu} \int_{-2 \nu}^{0} d t S_{\mu \mu^{\prime}} .
$$

Podemos reescrever $S_{\mu}^{\mu}$ em termos das variáveis de Mandelstan dadas na equação(1,4.43):

$$
\begin{aligned}
S_{\mu}^{\mu} & =-8\left(\frac{2 \nu-Q^{2}}{t}+\frac{t}{2 \nu-Q^{2}}+\frac{2 Q^{2}(2 \nu+t)}{t\left(2 \nu-Q^{2}\right)}\right) \\
& =-8\left(\frac{2 \nu-Q^{2}}{t}+\frac{4 Q^{2} \nu}{t\left(2 \nu-Q^{2}\right)}+\frac{t+2 Q^{2}}{2 \nu-Q^{2}}\right) \\
& =-8\left(\frac{\left(2 \nu-Q^{2}\right)^{2}+4 \nu Q^{2}}{t\left(2 \nu-Q^{2}\right)}+\frac{t+2 Q^{2}}{2 \nu-Q^{2}}\right) .
\end{aligned}
$$

Ao integrarmos $S_{\mu}^{\mu}$ em $t$ vamos encontrar uma divergência logarítmica, que é o mesmo tipo de singularidade infravermelha encontrada quando se calcula a seção de choque de bremsstrahlung da QED. Para progredir apesar dessa divergência, vamos fazer a integral até $-\lambda^{2}$, ao invés de zero, no termo proporcional a $1 / t$. Assim a equação $(1,4.48)$ fica:

$$
W_{\mu}^{\mu}=-\frac{4}{3} g^{2} \frac{Q_{f}^{2}}{16 \pi^{2}} \frac{1}{\nu}\left(\frac{\left(2 \nu-Q^{2}\right)^{2}+4 \nu Q^{2}}{2 \nu-Q^{2}} \ln \frac{\lambda^{2}}{2 \nu}\right.
$$




$$
\left.-\frac{4 \nu^{2}}{2\left(2 \nu-Q^{2}\right)}+\frac{4 \nu Q^{2}}{2 \nu-Q^{2}}\right)
$$

Em termos de $x$, podemos escrever $\left(2 \nu-Q^{2}\right)^{2}+4 \nu Q^{2}=4 \nu^{2}+Q^{4}=4 \nu^{2}\left(1+x^{2}\right) \mathrm{e}$ $2 \nu-Q^{2}=2 \nu(1-x)$, ou seja,

$$
\begin{gathered}
W_{\mu}^{\mu}=-\frac{4}{3} \alpha_{s} \frac{Q_{f}^{2}}{4 \pi} \frac{1}{\nu}\left(2 \nu \frac{1+x^{2}}{1-x} \ln \frac{\lambda^{2} x}{Q^{2}}-\frac{\nu}{1-x}+\frac{2 Q^{2}}{1-x}\right) \\
W_{\mu}^{\mu}=-\frac{4}{3} \alpha_{s} \frac{Q_{f}^{2}}{4 \pi}\left(2 \frac{1+x^{2}}{1-x} \ln \frac{\lambda^{2} x}{Q^{2}}-\frac{1}{1-x}+\frac{4 x}{1-x}\right)
\end{gathered}
$$

onde $\alpha_{s}=g^{2} /(4 \pi)$. De forma análoga obtemos

$$
\begin{aligned}
p^{\mu} p^{\mu^{\prime}} W_{\mu \mu^{\prime}} & =-\frac{4}{3} g^{2} \frac{Q_{f}^{2}}{16 \pi^{2}} \frac{1}{2 \nu}\left(4 \nu^{2}-\frac{4 \nu^{2}}{2}\right) \\
& =-\frac{4}{3} \alpha_{s} \frac{Q_{f}^{2}}{4 \pi} \nu .
\end{aligned}
$$

Por outro lado, a forma geral do tensor $W_{\mu \mu^{\prime}}$ é

$$
\begin{gathered}
W_{\mu \mu^{\prime}}=\left(-g_{\mu \mu^{\prime}}+\frac{q_{\mu} q_{\mu^{\prime}}}{q^{2}}\right) F_{1}^{Q}+\frac{1}{\nu}\left(p_{\mu}\right. \\
\left.-q_{\mu} \frac{q \cdot p}{q^{2}}\right) \cdot\left(p_{\mu^{\prime}}-q_{\mu^{\prime}} \frac{q \cdot p}{q^{2}}\right) F_{2}^{Q} .
\end{gathered}
$$

Portanto

$$
\begin{aligned}
W_{\mu}^{\mu} & =(-4+1) F_{1}^{Q}+\frac{1}{\nu}\left(p^{2}-2 \frac{(p \cdot q)^{2}}{q^{2}}+\frac{(p \cdot q)^{2}}{q^{2}}\right) F_{2}^{Q} \\
& =-3 F_{1}^{Q}+\frac{\nu}{Q^{2}} F_{2}^{Q}=-3 F_{1}^{Q}+\frac{F_{2}^{Q}}{2 x}
\end{aligned}
$$


e

$$
\begin{aligned}
p^{\mu} p^{\mu^{\prime}} W_{\mu \mu^{\prime}} & =\left(-p^{2}+\frac{(p \cdot q)^{2}}{q^{2}}\right) F_{1}^{Q}+\frac{1}{\nu}\left(p^{2}-\frac{(p . q)^{2}}{q^{2}}\right)^{2} F_{2}^{Q} \\
& =-\frac{\nu^{2}}{Q^{2}} F_{1}^{Q}+\frac{\nu^{3}}{Q^{4}} F_{2}^{Q} \\
& =Q^{2}\left(-\frac{F_{1}^{Q}}{4 x^{2}}+\frac{F_{2}^{Q}}{8 x^{3}}\right) \\
& =\frac{Q^{2}}{4 x^{2}}\left(\frac{F_{2}^{Q}}{2 x}-F_{1}^{Q}\right)
\end{aligned}
$$

onde usamos $x=Q^{2} /(2 \nu)$.

Como a relação de Callan-Gross dá $F_{2}^{Q}=2 x F_{1}^{Q}$ o valor de $p^{\mu} p^{\mu^{\prime}} W_{\mu \mu^{\prime}}$ é uma medida da violação dessa relação. Usando as eqs. $(1,4.51)$ e $(1,4.52)$ podemos eliminar $F_{1}^{Q}$ de tal forma a obter

$$
W_{\mu}^{\mu}-\frac{12 x^{2}}{Q^{2}} p^{\mu} p^{\mu^{\prime}} W_{\mu \mu^{\prime}}=\frac{F_{2}^{Q}}{2 x}-\frac{3 F_{2}^{Q}}{2 x}=-\frac{F_{2}^{Q}}{x},
$$

então

$$
F_{2}^{Q}=-x W_{\mu}^{\mu}+\frac{12 x^{3}}{Q^{2}} p^{\mu} p^{\mu^{\prime}} W_{\mu \mu^{\prime}}
$$

Das equações $(1,4.52)$ e $(1,4.50)$ vemos que

$$
F_{2}^{Q}-2 x F_{1}^{Q}=\frac{8 x^{3}}{Q^{2}} p^{\mu} p^{\mu^{\prime}} W_{\mu \mu^{\prime}}=-\frac{4}{3} \alpha_{s} Q_{f}^{2} \frac{x^{2}}{\pi}
$$

e usando as equações $(1,4.50)$ e $(1,4.49)$ na equação $(1,4.53)$ temos

$$
\begin{aligned}
F_{2}^{Q}\left(x, Q^{2}\right) & =-\frac{4}{3} \alpha_{s} Q_{f}^{2}\left[\frac{3}{2} \frac{x^{2}}{\pi}-\frac{x}{4 \pi}\left(2 \frac{1+x^{2}}{1-x} \ln \frac{\lambda^{2} x}{Q^{2}}\right.\right. \\
& \left.\left.-\frac{1}{1-x}+\frac{4 x}{1-x}\right)\right] .
\end{aligned}
$$


Definindo $\Delta F_{2}^{Q}=F_{2}^{Q}\left(x, Q_{1}^{2}\right)-F_{2}^{Q}\left(x, Q_{2}^{2}\right)$, encontramos

$$
\Delta F_{2}^{Q}=-\frac{4}{3} \alpha_{s} Q_{f}^{2} \frac{x}{2 \pi} \frac{1+x^{2}}{1-x} \ln \frac{Q_{1}^{2}}{Q_{2}^{2}}
$$

Vamos agora investigar a dependência das funções de estrutura do núcleon $F_{1,2}$ com as funções de estutura dos quarks $F_{1,2}^{Q}$. No modelo de quarks livres vimos que o "scaling" fornecia para o núcleon

$$
F_{1}\left(X, Q^{2}\right)=F_{1}(X), \quad ; F_{2}\left(X, Q^{2}\right)=F_{2}(X)
$$

com $F_{2}=2 X F_{1}$ que é a relação de Callan-Gross. Além disso, vimos que podemos escrever

$$
F_{1}(X)=\frac{1}{2} \sum_{i} Q_{i}^{2} f_{i}(X), \quad F_{2}(X)=\sum_{i} Q_{i}^{2} X f_{i}(X)
$$

onde $Q_{i}$ é a carga do parton $i$ e $f_{i}(X)$ sua função de distribuição, tal que:

$$
\int_{0}^{1} f_{i}(X) d X=1
$$

onde $f_{i}(X)$ é uma probabilidade. Das definições acima vemos que podemos reescrever $F_{2}$ como:

$$
F_{2}(X)=\sum_{i} \int_{0}^{1} d \xi_{i} Q_{i}^{2} \xi_{i} f_{i}\left(\xi_{i}\right) \delta\left(\xi_{i}-X\right) .
$$

Existem duas variáveis: $X=Q^{2} /\left(2 P_{N} \cdot q\right)$ e $x=Q^{2} /(2 p \cdot q)$, onde $P_{N}$ é o momento do núcleon e $p$ o momento do quark. Na equação $(1,4.55), \xi_{i}$ é a fração do momento do núcleon carregada pelo parton $i: X=\xi_{i} x$.

Por definição as funções de estrutura são fatores do tensor de espalhamento, que transformam as contribuições das partículas puntiformes nas de partículas extensas. Assim, como nosso cálculo forneceu um $F_{2}^{Q}$ para o quark, a equação $(1,4.55)$ deve ser 
modificada como:

$$
F_{2}\left(X, Q^{2}\right)=\sum_{i} \int_{0}^{1} d \xi_{i} f_{i}\left(\xi_{i}\right) \xi_{i} \int_{0}^{1} d x \delta\left(X-x \xi_{i}\right) F_{2}^{Q}\left(x, Q^{2}\right)
$$

e assim vemos a quebra do "scaling" uma vez que $F_{2}$ é agora função explícita de $Q^{2}$ e $x$.

Para uma partícula puntiforme: $F_{2}^{Q, i}(x)=Q_{i}^{2} \delta(x-1)$ e, portanto, a equação $(1,4.55)$ é recuperada. Como $Q_{i}^{2}$ já está dentro de $F_{2}^{Q}$, ela não aparece explicitamente em $(1,4.56)$. Assim, usando a equação $(1,4.54)$ em $(1,4.56)$, temos

$$
\begin{aligned}
\Delta F_{2}\left(X, Q_{1}^{2}, Q_{2}^{2}\right)= & -\frac{4}{3} \frac{\alpha_{s}}{2 \pi} \sum_{i} Q_{i}^{2} \int_{0}^{1} d \xi_{i} f_{i}\left(\xi_{i}\right) \xi_{i} \int_{0}^{1} d x \\
& x \frac{1+x^{2}}{1-x} \ln \frac{Q_{1}^{2}}{Q_{2}^{2}} \delta\left(X-x \xi_{i}\right),
\end{aligned}
$$

$\operatorname{como} \delta\left(X-x \xi_{i}\right)=\frac{1}{\xi_{i}} \delta\left(x-X / \xi_{i}\right)$ e como devido a essa função delta $X / \xi_{i}<1 \Rightarrow \xi_{i}>X$, fazendo a integral em $x$ obtemos

$$
\begin{aligned}
\Delta F_{2}\left(X, Q_{1}^{2}, Q_{2}^{2}\right)= & -\frac{4}{3} \frac{\alpha_{s}}{2 \pi} \ln \frac{Q_{1}^{2}}{Q_{2}^{2}} \sum_{i} Q_{i}^{2} \int_{X}^{1} d \xi_{i} f_{i}\left(\xi_{i}\right)\left(\frac{X}{\xi_{i}}\right. \\
& \left.\frac{1+\left(X / \xi_{i}\right)^{2}}{1-X / \xi_{i}}\right) .
\end{aligned}
$$

Vemos que para $\xi_{i}=X, x$ é igual a 1 e essa integral tem uma divergência. Como $x=1$ significa espalhamento elástico, o glúon emitido nesse processo não carrega nem energia nem momento.

$\mathrm{Na}$ QED pode-se mostrar que essas divergências se cancelam quando todos os processos de espalhamento, incluindo elástico, são considerados. Na QCD essa prova é mais sofisticada, mas essa divergência pode ser cancelada. Assim, incluindo a correção 
de bremsstraklung que elimina a divergência ficamos com

$$
\begin{aligned}
& \left.\Delta F_{2} \text { (bremsstrahlung }+ \text { correções radiativas }\right) \approx \\
& -\frac{4}{3} \frac{\alpha_{s}}{2 \pi} \ln \frac{Q_{1}^{2}}{Q_{2}^{2}} \sum_{i} Q_{i}^{2} \int_{X}^{1} d \xi_{i} f_{i}\left(\xi_{i}\right) \frac{X}{\xi_{i}}\left[\frac{1+\left(X / \xi_{i}\right)^{2}}{1-X / \xi_{i}}\right]_{+},
\end{aligned}
$$

onde $\left[\left(1+z^{2}\right) /(1-z)\right]_{+}$é definida por

$$
\int_{0}^{1} d z F(z)\left[\frac{1+z^{2}}{1-z}\right]_{+}=\int_{0}^{1} d z(F(z)-F(1)) \frac{1+z^{2}}{1-z}
$$

onde $z=X / \xi_{i}$. Para derivar as equações de Dokshitzer-Gribov-Lipatov-Altarelli-Parisi escrevemos

$$
\Delta F_{2}=\sum_{i} Q_{i}^{2} x \Delta f_{i}(x)
$$

e comparando com $(1,4.57)$, temos que ("re-batizando" $X$ como $x$ )

$$
\begin{aligned}
\sum_{i} Q_{i}^{2} x \Delta f_{i}(x)= & -\frac{\alpha_{s}}{2 \pi} \ln \frac{Q_{1}^{2}}{Q_{2}^{2}} \sum_{i} Q_{i}^{2} \int_{x}^{1} d \xi_{i}\left[f_{i}\left(\xi_{i}\right)\right. \\
& \left.\frac{x}{\xi_{i}} P_{q q}\left(x / \xi_{i}\right)\right]
\end{aligned}
$$

onde definimos

$$
P_{q q}(z)=\frac{4}{3}\left[\frac{1+z^{2}}{1-z}\right]_{+} .
$$

Como os sabores diferentes são completamente independentes, podemos identificar as expressões na equação acima termo a termo, portanto

$$
\Delta f(x)=-\frac{\alpha_{s}}{2 \pi} \ln \frac{Q_{1}^{2}}{Q_{2}^{2}} \int_{x}^{1} \frac{d y}{y} f(y) P_{q q}\left(\frac{x}{y}\right)
$$

onde omitimos o índice $i$. Da equação acima fica claro que agora $f$ é também função explícita de $Q^{2}$. Definindo a variável momento logarítmico

$$
t\left(Q^{2}\right)=\ln \frac{Q^{2}}{Q_{0}^{2}} \Rightarrow \Delta t=t\left(Q_{2}^{2}\right)-t\left(Q_{1}^{2}\right)=\ln \frac{Q_{2}^{2}}{Q_{1}^{2}}
$$


obtemos

$$
\frac{\Delta f}{\Delta t}=\frac{\alpha_{s}}{2 \pi} \int_{x}^{1} \frac{d y}{y} f(y) P_{q q}\left(\frac{x}{y}\right)
$$

que só é válida para $\Delta t$ pequeno. Para valores grandes de $\Delta t$ temos que levar em conta o fato de que $\alpha_{s}$ também depende de $Q^{2}$ e, portanto, a equação correta fica

$$
\frac{\partial f(x, t)}{\partial t}=\frac{\alpha_{s}(t)}{2 \pi} \int_{x}^{1} \frac{d y}{y} f(y, t) P_{q q}\left(\frac{x}{y}\right)
$$

que é uma das equações de DGLAP. A função $P_{q q}$ é conhecida como função de "splitting" e é interpretada como a probabilidade de que um quark mostre sua estrutura interna quark+gluon numa resolução $t=\ln Q^{2} / Q_{0}^{2}$. Correções adicionais da QCD se somam ao lado direito dessa última equação. Essa equação descreve a taxa de mudança das funções de estrutura dos partons como função de $t$ e dão uma medida da violação de scaling da função de estrutura $F_{2}$. Dada a função de estrutura do quark em algum ponto de referência $\left(x, Q^{2}\right)$, podemos calculá-la em qualquer outro valor de $Q^{2}$ usando a equação de DGLAP.

Experimentalmente se observou que a violação de scaling de fato acontece, e que $F_{2}$ se torna uma função de $x$ e $Q^{2}$. Para $x>0.5, F_{2}$ diminui com o aumento de $Q^{2}$, enquanto que para $x<0.1 F_{2}$ aumenta com $Q^{2}$. Esse comportamento é muito bem descrito pela equação de DGLAP, mesmo em ordem dominante, como pode ser visto nas figuras 1.6 e 1.7 .

A QCD perturbativa permite determinar somente a evolução das distribuições partônicas através das equações DGLAP. As condições iniciais para a evolução são de natureza não-perturbativa, devendo ser extraídas do experimento para uma dada virtualidade do fóton. Como as distribuições partônicas são universais, independentes do processo considerado, após a determinação em uma dada condição inicial podemos com o auxílio das equações DGLAP determinar seu valor para outra virtualidade e assim utilizá-las no cálculo do processo de interesse. Atualmente, existem na literatura diversos grupos que se dedicam a obter parametrizações para as distribuições partônicas. Nesta tese, nos resultados referentes à produção de hádrons, utilizaremos 


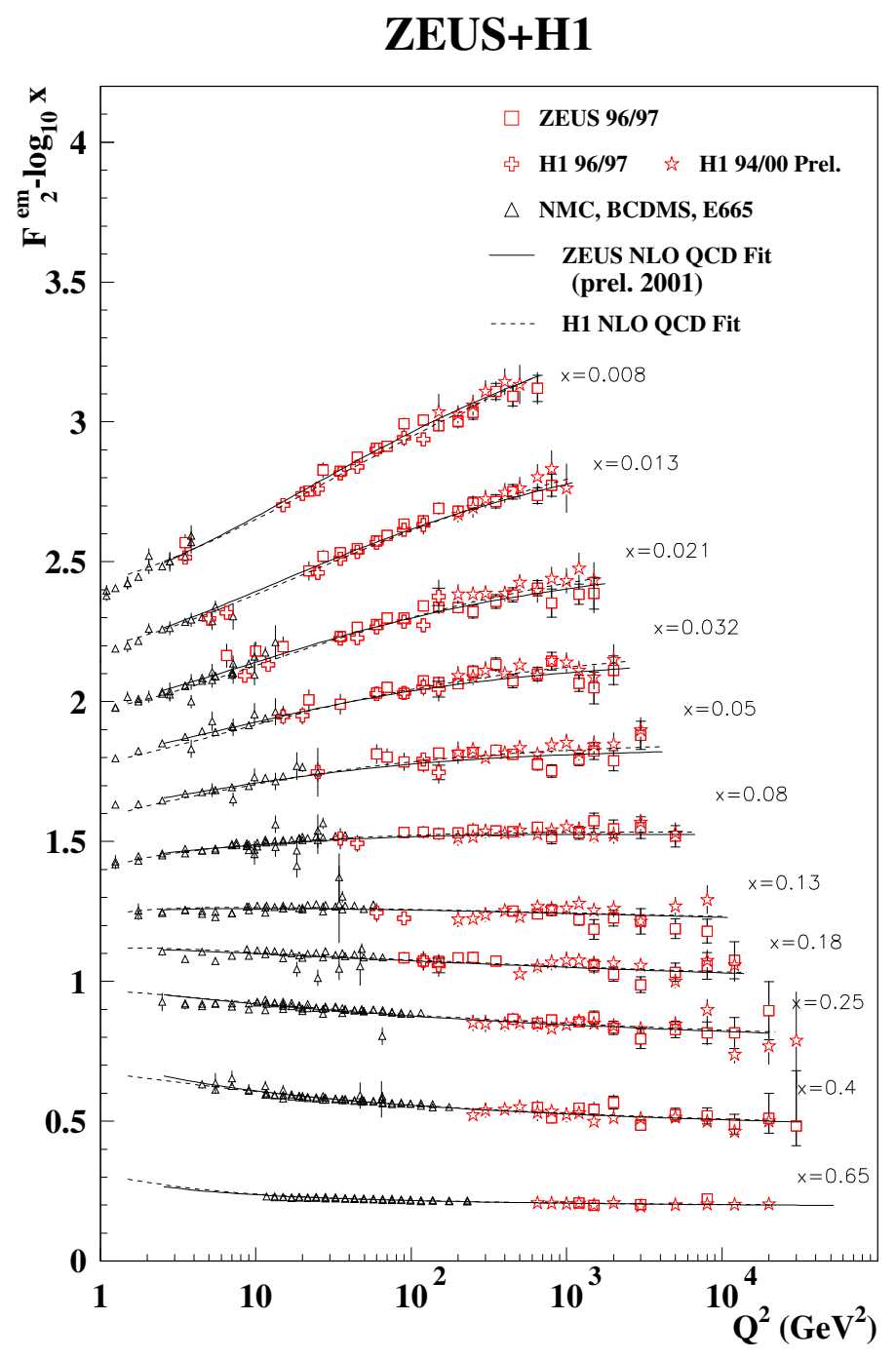

Figura 1.6: Função de estrutura $F_{2}$ (Extraído de [21]).

a parametrização CTEQ5 [18]. Na figura 1.8 apresentamos a distribuição de partons segundo a parametrização CTEQ5 em função da variável $x$, onde observamos o forte crescimento da distribuição de glúons na região de pequeno $x$.

Vimos um exemplo de como derivar a equação DGLAP para os quarks de valência. Para uma distribuição completa temos que a distribuição de quarks acopla-se à distribuição de glúons, sendo a evolução dada por [12, 19]

$$
\frac{\partial q_{S}\left(x, Q^{2}\right)}{\partial \ln Q^{2}}=\frac{\alpha_{s}}{2 \pi}\left[\int_{x}^{1} \frac{d x_{1}}{x_{1}}\left(P_{q q}\left(\frac{x}{x_{1}}\right) q_{S}\left(x_{1}, Q^{2}\right)+P_{q g}\left(\frac{x}{x_{1}}\right) g\left(x_{1}, Q^{2}\right)\right)\right]
$$




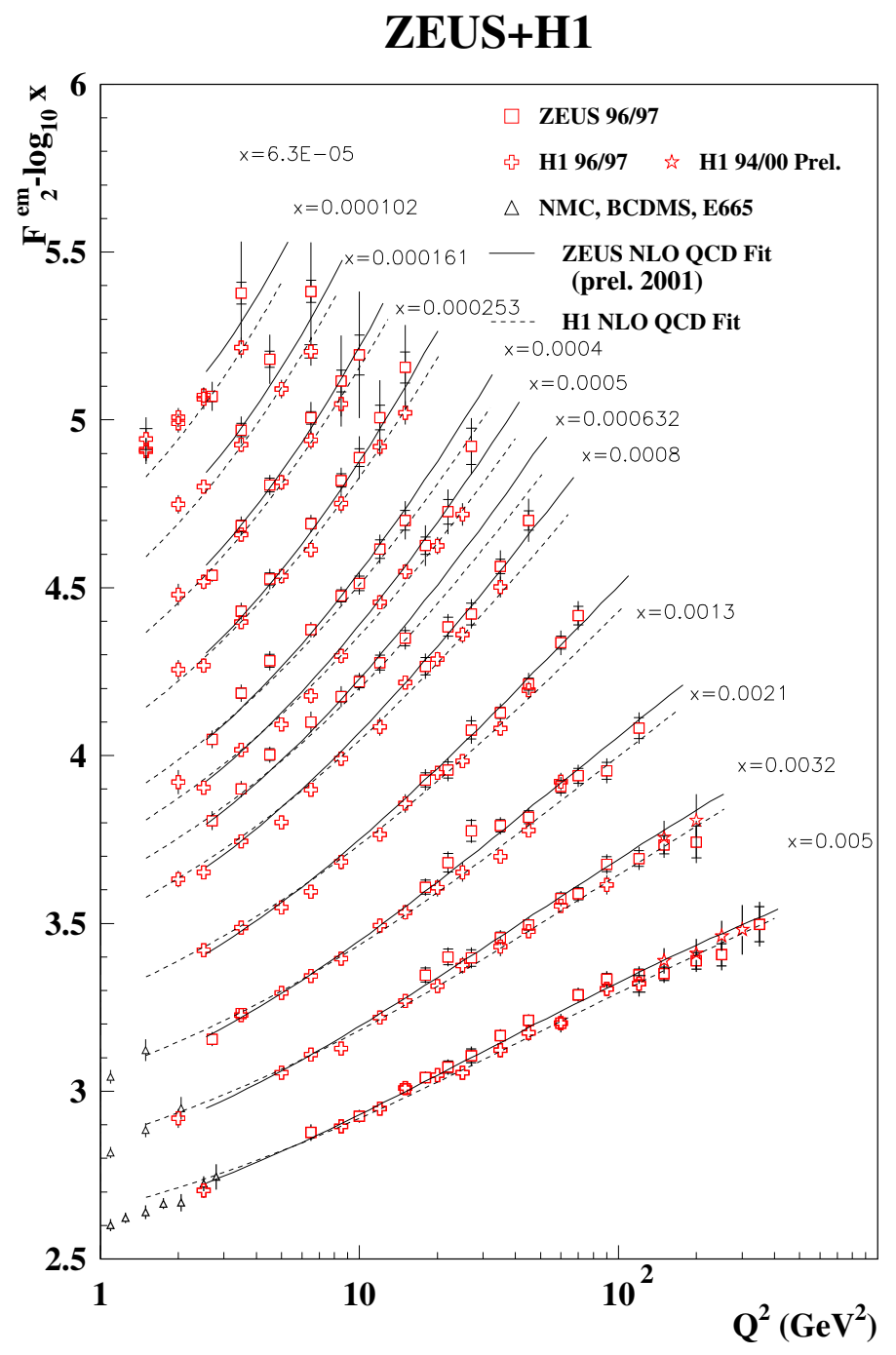

Figura 1.7: Função de estrutura $F_{2}$ (Extraído de [21]).

onde $q_{S}$ é a distribuição de quarks singleto:

$$
\sum\left(x, Q^{2}\right)=\sum_{i}\left[q_{i}\left(x, Q^{2}\right)+\bar{q}_{i}\left(x, Q^{2}\right)\right]
$$

onde $\sum_{i}$ representa a soma sobre os sabores. É possível escrever uma equação de evolução para glúons, e essa é dada por:

$$
\frac{\partial g\left(x, Q^{2}\right)}{\partial \ln Q^{2}}=\frac{\alpha_{s}}{2 \pi}\left[\int_{x}^{1} \frac{d x_{1}}{x_{1}}\left(P_{g q}\left(\frac{x}{x_{1}}\right) q_{S}\left(x_{1}, Q^{2}\right)+P_{g g}\left(\frac{x}{x_{1}}\right) g\left(x_{1}, Q^{2}\right)\right)\right] .
$$




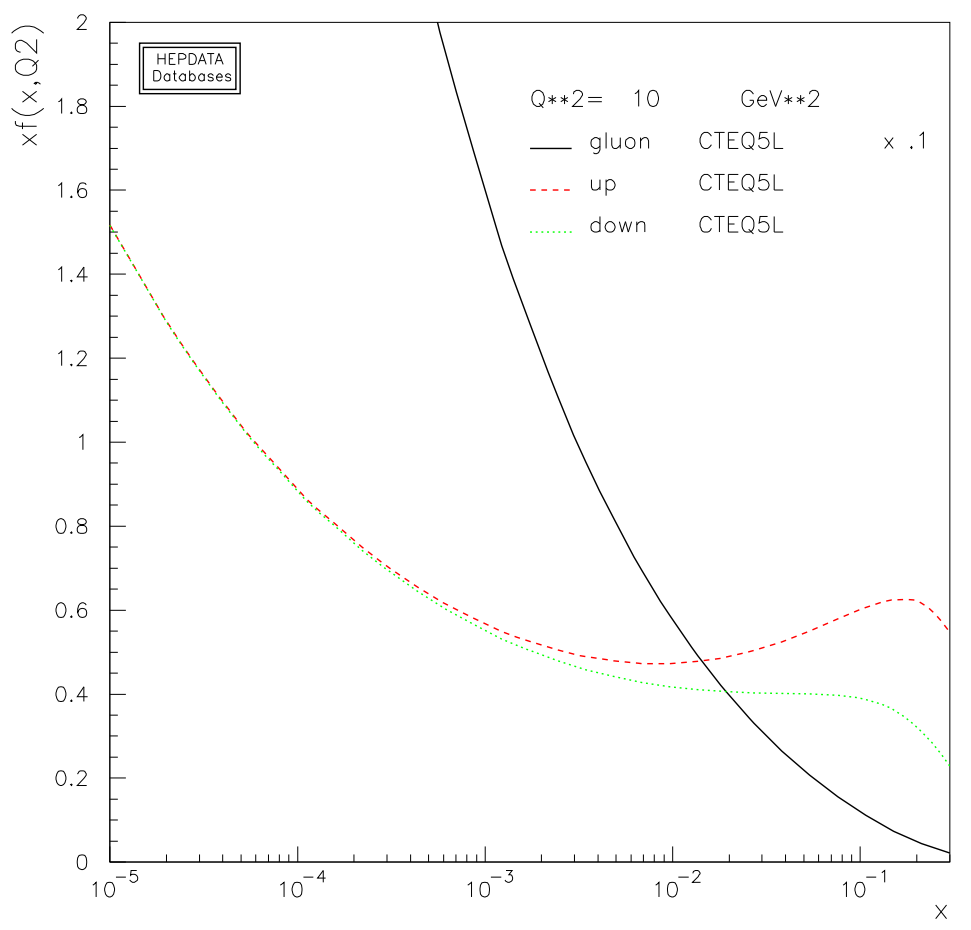

Figura 1.8: Distribuição de partons segundo a parametrização CTEQ5 [18].

As funções $P_{i j}\left(P_{q q}, P_{q g}, P_{g q}, P_{g g}\right)$ são denominadas funções de splitting. Em ordem dominante de $\alpha_{s}$ as funções de splitting são dadas por:

$$
\begin{aligned}
& P_{q q}^{(0)}(z)=C_{F}\left[\frac{1+z^{2}}{(1-z)_{+}}+\frac{3}{2} \delta(1-z)\right] \\
& P_{q g}^{(0)}(z)=\frac{1}{2}\left[z^{2}+(1-z)^{2}\right] \\
& P_{g q}^{(0)}(z)=C_{F}\left[\frac{1+(1-z)^{2}}{z}\right] \\
& P_{g g}^{(0)}(z)=2 C_{A}\left[\frac{z}{(1-z)_{+}}+\frac{1-z}{z}+z(1-z)\right]+\frac{11 C_{A}-2 n_{f}}{6} \delta(1-z)
\end{aligned}
$$

onde $C_{F}=\left(N_{C}^{2}-1\right) / 2 N_{C}, C_{A}=N_{C}$ e $n_{f}$ é o número de sabores, e identificamos também $z=x / x_{1}$. Sendo as distribuições "+" dada por:

$$
\int_{0}^{1} d x \frac{f(x)}{(1-x)_{+}}=\int_{0}^{1} d x \frac{f(x)-f(1)}{1-x}
$$


Existe uma outra equação de evolução, em que o momento transferido $Q^{2}$, é fixado e a distribuição de glúons é evoluída em energia, ou seja, em termos de $\log (1 / x)$, sendo essa denominada evolução BFKL. No Apêndice há uma derivação da solução dessa equação e uma discussão mais detalhada é apresentada no Capítulo 3.

\subsection{A dinâmica DGLAP para pequeno $x$}

Consideremos as equações DGLAP apresentadas na seção anterior. A partir da análise das funções de splitting $P_{i j}$ verifica-se que as funções associadas ao setor de glúons $P_{g q}(z)$ e $P_{g g}(z)$ são singulares para $z \rightarrow 0$, e/ou $x \rightarrow 0$, pois como definido anteriormente $z=\frac{x}{x_{1}}$. As demais funções de splitting, associadas ao setor de quarks, são regulares neste limite. Consequentemente, o comportamento das distribuições partônicas para pequeno $x$ é determinado pela dinâmica gluônica.

Em vista do que foi dito acima, podemos aproximar a equação de DGLAP para $g\left(x, Q^{2}\right)$ na região de pequeno $x$ por:

$$
\frac{\partial g\left(x, Q^{2}\right)}{\partial \ln Q^{2}}=\frac{\alpha_{s}}{2 \pi} \int_{x}^{1} \frac{d x_{1}}{x_{1}} \mathcal{P}_{g g}\left(\frac{x}{x_{1}}\right) g\left(x_{1}, Q^{2}\right),
$$

onde $\mathcal{P}_{g g}(z)=P_{g g}^{z \rightarrow 0}(z)=\frac{2 C_{A}}{z}$ representa o termo singular na função de splitting. Reescrevendo a equação $(1,5.65)$.

$$
\begin{gathered}
\frac{\partial g\left(x, Q^{2}\right)}{\partial \ln Q^{2}}=\frac{\alpha_{s}}{2 \pi} \int_{x}^{1} \frac{d x_{1}}{x_{1}} \frac{2 C_{A}}{z} g\left(x_{1}, Q^{2}\right), \\
g\left(x, Q^{2}\right)=\frac{\alpha_{s} C_{A}}{\pi} \int_{x}^{1} \frac{d x_{1}}{x_{1}} \frac{1}{z} \int_{Q_{0}^{2}}^{Q^{2}} \frac{d k_{T}^{2}}{k_{T}^{2}} g\left(x_{1}, k_{T}^{2}\right),
\end{gathered}
$$

ou ainda:

$$
g\left(x, Q^{2}\right)=\frac{\alpha_{s} C_{A}}{\pi} \int_{x}^{1} \frac{d x_{1}}{x_{1}} \frac{x_{1}}{x} \int \frac{d k_{T}^{2}}{k_{T}^{2}} g\left(x_{1}, k_{T}^{2}\right)
$$


Portanto,

$$
x g\left(x, Q^{2}\right)=\int_{x} \frac{d x_{1}}{x_{1}} \int_{Q_{0}^{2}}^{Q^{2}} \frac{d k_{T}^{2}}{k_{T}^{2}} \frac{\alpha_{s} N_{c}}{\pi} x_{1} g\left(x_{1}, k_{T}^{2}\right)
$$

onde introduzimos um corte $Q_{0}$ na integração sobre os momentos transversais para separar a contribuição não perturbativa, e usamos que $C_{A}=N_{c}$. Definindo $y=$ $\ln (1 / x)$ e $\Gamma=\ln \left(Q^{2} / Q_{0}^{2}\right)$ temos:

$$
x g\left(x, Q^{2}\right)=\int_{y} d y^{\prime} \int_{\Gamma} d \Gamma^{\prime} \frac{\alpha_{s} N_{c}}{\pi} x^{\prime} g\left(x^{\prime}, k^{2}\right)
$$

A solução da equação $(1,5.70)$ pode ser obtida facilmente quando consideramos que a constante de acoplamento está fixa. Introduzimos a transformada de Mellin de $x g\left(x, Q^{2}\right) \equiv G(y, \Gamma)$, definida por

$$
G(\omega, \Gamma)=\int d y e^{\omega y} G(y, \Gamma)
$$

e sua inversa por

$$
G(y, \Gamma)=\frac{1}{2 \pi i} \int_{-i \infty}^{+i \infty} d \omega e^{\omega y} G(\omega, \Gamma)
$$

onde a integração de contorno localiza-se à direita de todas as singularidades de $G(\omega, \Gamma)$, sendo $\omega$ a variável conjugada a $y[19]$. Assim,

$$
\begin{aligned}
G(\omega, \Gamma) & =\int d y e^{\omega y} \int_{y} d y^{\prime} \int_{\Gamma} d \Gamma^{\prime} \frac{\alpha_{s} N_{c}}{\pi} G\left(y^{\prime}, \Gamma^{\prime}\right) \\
& =\frac{\alpha_{s} N_{c}}{\pi} \int d y^{\prime} \int_{\Gamma} d \Gamma^{\prime} \int d y e^{\omega y} \Theta\left(y^{\prime}-y\right) G\left(y^{\prime}, \Gamma^{\prime}\right) \\
& =\frac{\alpha_{s} N_{c}}{\omega \pi} \int d y^{\prime} \int_{\Gamma} d \Gamma^{\prime} e^{\omega y^{\prime}} G\left(y^{\prime}, \Gamma^{\prime}\right) \\
& =\frac{\alpha_{s} N_{c}}{\omega \pi} \int_{\Gamma} d \Gamma^{\prime} \int d y^{\prime} e^{\omega y^{\prime}} G\left(y^{\prime}, \Gamma^{\prime}\right) \\
& =\frac{\alpha_{s} N_{c}}{\omega \pi} \int_{\Gamma} d \Gamma^{\prime} G\left(\omega, \Gamma^{\prime}\right) .
\end{aligned}
$$


Derivando a expressão acima com respeito a $\Gamma^{\prime}$, obtemos:

$$
\begin{aligned}
\frac{d G}{d \Gamma^{\prime}} & =\frac{\alpha_{s} N_{c}}{\omega \pi} G\left(\omega, \Gamma^{\prime}\right) \\
\frac{d G}{G} & =\frac{\alpha_{s} N_{c}}{\omega \pi} d \Gamma^{\prime} .
\end{aligned}
$$

A solução desta equação é

$$
G(\omega, \Gamma)=G\left(\omega, \Gamma_{0}\right) e^{\frac{\alpha_{S} N_{c}}{\pi \omega} \Gamma}
$$

O termo $\frac{\alpha_{s} N_{c}}{\pi \omega}$ é denominado dimensão anômala. O crescimento da distribuição de glúons na região de pequeno $x$ (ou pequeno $\omega$ ) está associado ao fato de que a dimensão anômala torna-se muito grande para $\omega \rightarrow 0$. Agora, para obter a distribuição de glúons fazemos a transformada inversa de Mellin

$$
G(y, \Gamma)=\frac{1}{2 \pi i} \int d \omega e^{\omega y} G\left(\omega, \Gamma_{0}\right) e^{\frac{\alpha_{s} N_{c}}{\pi \omega} \Gamma}
$$

Na região de pequeno $x$ (grande $y$ ) e grande $\Gamma$ podemos utilizar o método de ponto de sela no cálculo da integral (1,5.76). Escrevendo por extenso o expoente de $(1,5.76)$ temos:

$$
u=\omega y+\frac{\alpha_{s} N_{c}}{\pi \omega} \Gamma
$$

O ponto de sela é determinado a partir da condição de mínimo, ou seja,

$$
\frac{\partial u}{\partial \omega}=0
$$

assim o ponto de sela $\omega_{s}$ é:

$$
\omega_{s}=\sqrt{\frac{\alpha_{s} N_{c} \Gamma}{\pi y}} .
$$


Expandindo $u$ até segunda ordem em torno do ponto de sela $\omega_{s}$ :

$$
u=u\left(\omega_{s}\right)+\left.\frac{\partial u}{\partial \omega}\right|_{\omega_{s}}\left(\omega-\omega_{s}\right)+\left.\frac{1}{2} \frac{\partial^{2} u}{\partial \omega^{2}}\right|_{\omega_{s}}\left(\omega-\omega_{s}\right)^{2}+\ldots
$$

A segunda derivada calculada em $\omega_{s}$ é:

$$
\left.\frac{\partial^{2} u}{\partial \omega^{2}}\right|_{\omega_{s}}=\left.\frac{2 \alpha_{s} N_{C}}{\pi \omega^{3}} \Gamma\right|_{\omega_{s}}=\frac{2 \alpha_{s} N_{C}}{\pi \omega_{s}^{3}} \Gamma
$$

Substituindo a expansão acima em (1,5.76), obtemos

$$
G(y, \Gamma)=\frac{1}{2 \pi i} \int_{-i \infty}^{+i \infty} d \omega G\left(\omega, \Gamma_{0}\right) \exp \left[u\left(\omega_{s}\right)+\frac{1}{2} u^{\prime \prime}\left(\omega-\omega_{s}\right)^{2}\right]
$$

fazendo a seguinte substituição: $\omega-\omega_{s}=i \nu \Rightarrow d \omega=i d \nu$,

$$
G(y, \Gamma)=\frac{1}{2 \pi} \int_{-\infty}^{+\infty} d \nu G\left(\omega_{0}, \Gamma_{0}\right) \exp \left[u\left(\omega_{s}\right)+\frac{1}{2} u^{\prime \prime}\left(-\nu^{2}\right)\right]
$$

onde identificamos uma integral gaussiana. Resolvendo, chegamos em

$$
\begin{aligned}
G(y, \Gamma) & =\frac{G\left(\omega_{0}, \Gamma_{0}\right)}{2 \pi} \sqrt{\frac{\pi}{\frac{1}{2} u^{\prime \prime}}} \exp \left(u\left(\omega_{s}\right)\right) \\
& =\frac{G\left(\omega_{0}, \Gamma_{0}\right)}{2 \pi} \sqrt{\frac{\pi^{2} \omega_{s}^{3}}{\alpha_{s} N_{C} \Gamma}} \exp \left[\omega_{s} y+\frac{\alpha_{s} N_{c}}{\pi \omega_{s}} \Gamma\right]
\end{aligned}
$$

Finalmente substituindo o valor de $\omega_{s}$, chegamos a

$$
G(y, \Gamma)=G\left(\omega_{0}, \Gamma_{0}\right) \frac{\alpha_{s} N_{c}}{\pi} \frac{\Gamma^{\frac{1}{4}}}{\left(\frac{\alpha_{s} N_{c}}{\pi} y\right)^{\frac{3}{4}}} e^{2 \sqrt{\frac{\alpha_{s} N_{c}}{\pi} \Gamma y}}
$$

onde toda a dependência na condição inicial está concentrada na função $G\left(\omega_{0}, \Gamma_{0}\right)$, sendo esta a solução da DGLAP na aproximação de duplo logarítmo dominante (DLLA). Observamos que a equação DGLAP prevê um forte crescimento da distribuição de glúons na região de pequeno $x$. 


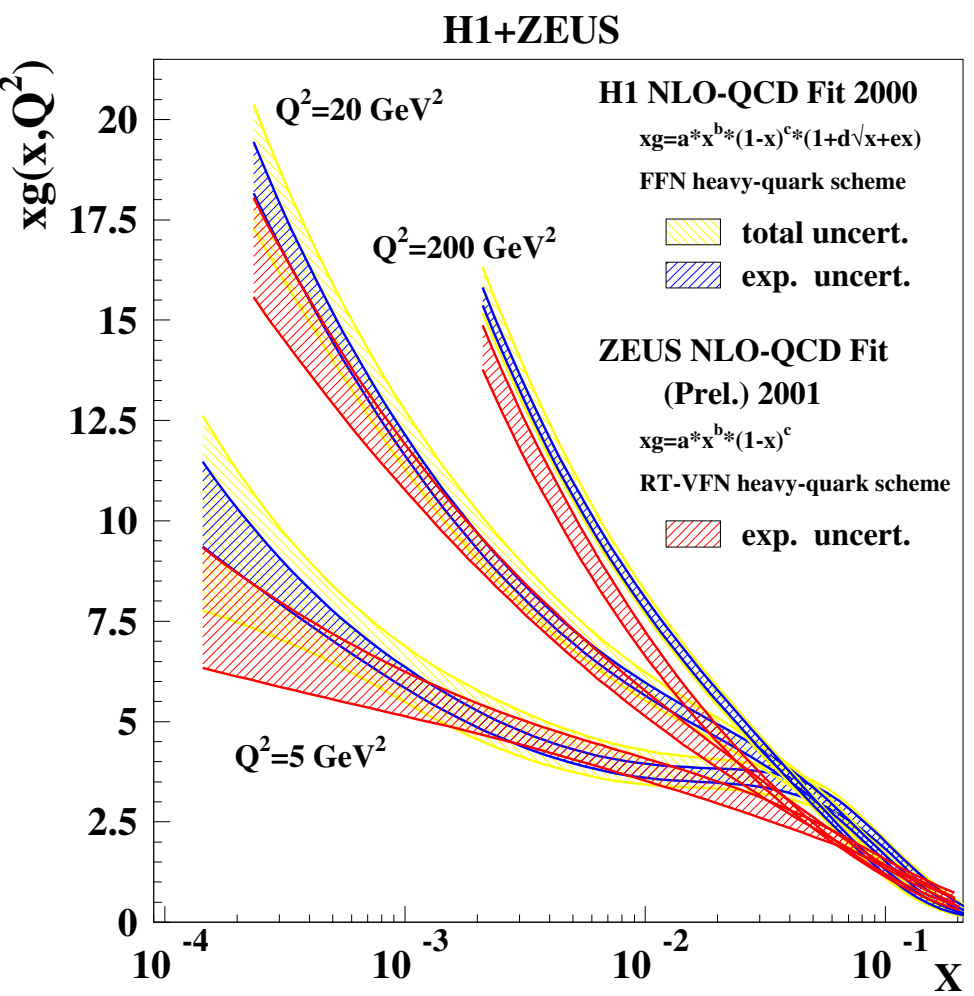

Figura 1.9: Resultados de ZEUS para a distribuição de glúons. Extraído

\subsection{Saturação}

Como foi visto nas seções anteriores, em espalhamento elétron-próton a altas energias e momentos transferidos (espalhamento inelástico profundo - DIS) podemos sondar o interior do próton e determinar as propriedades de seus constituintes. Em particular como foi discutido na seção anterior, um dos resultados mais surpreendentes deste estudo é que o número de glúons observados no interior do próton cresce à medida em que aumentamos a energia do projétil e/ou a virtualidade $Q^{2}$ do fóton emitido pelo elétron. A figura 1.9 mostra a distribuição de glúons como função de $x$ para vários valores de $Q^{2}$ extraída pela colaboração ZEUS a partir de seus resultados experimentais. O crescimento do número de glúons pode ser entendido como um efeito cascata, onde os próprios glúons se dividem em dois ou três, além de serem também emitidos pelos quarks.

Em colisões próton-próton, o alvo é sondado não por fótons mas por glúons do 
projétil. Nestas colisões hadrônicas, à medida em que a energia aumenta, também deve ser observado um crescimento do número de glúons, ou mais precisamente, da função de distribuição dos glúons $x g\left(x, Q^{2}\right)$. Este crescimento faz com que um próton, observado a altas energias, seja visto como um meio denso e colorido.

Em 1983, Gribov, Levin e Ryskin [20] apresentaram a idéia de que a energias muito altas o número de glúons seria tão grande que o processo de recombinação de glúons, $g+g \rightarrow g$, passaria a ser importante nas equações de evolução. Este processo reduz o crescimento da densidade de glúons produzindo um efeito que foi chamado de "saturação perturbativa de partons". Em [20] foi proposta uma equação de evolução não-linear, na dupla aproximação logarítmica (DLLA), na qual $\ln (1 / x)>>1$ e $\ln \left(Q^{2}\right)>>1$. Esta equação leva em conta o efeito da recombinação de glúons:

$$
Q^{2} \frac{\partial^{2} x g\left(x, Q^{2}\right)}{\partial \ln (1 / x) \partial Q^{2}}=\frac{\alpha_{S} N_{c}}{\pi} x g\left(x, Q^{2}\right)-\frac{4 \alpha_{S}^{2} N_{c}}{3 C_{F} R^{2}} \frac{1}{Q^{2}}\left[x g\left(x, Q^{2}\right)\right]^{2}
$$

Observando o sinal negativo do termo não-linear, vemos que o crescimento acentuado de $x g\left(x, Q^{2}\right)$ é atenuado pelo termo responsável pela recombinação de glúons.

O trabalho pioneiro de Gribov, Levin e Ryskin gerou uma longa série de trabalhos cujo objetivo era desenvolver uma teoria da saturação. Uma teoria efetiva da QCD a altas energias e pequenos $x$ é o chamado "Color Glass Condensate"(CGC) [21] que leva às equações de evolução JIMWLK [22]. Uma teoria semelhante, mas com aproximações diferentes, foi desenvolvida por Balitsky e depois por Kovchegov. Dela é possível chegar à equação de evolução chamada de Balitsky-Kovchegov (BK). Estas equações mencionadas são muito complicadas e mesmo a mais simples delas (BK) só pode ser resolvida numericamente. Apresentaremos uma versão mais detalhada no Capítulo 3.

Quando comparamos a equação $(1,4.59)$, que é a equação de DGLAP para a distribuição de glúons, com a equação $(1,6.85)$, vemos claramente que a equação $(1,4.59)$ não incorpora a recombinação de glúons e dessa forma o número de glúons tende a crescer continuamente. Como não temos a dependência quadrática em $g(x)$ (que observamos na equação $(1,6.85))$ esta equação é chamada de equação linear de evolução. 


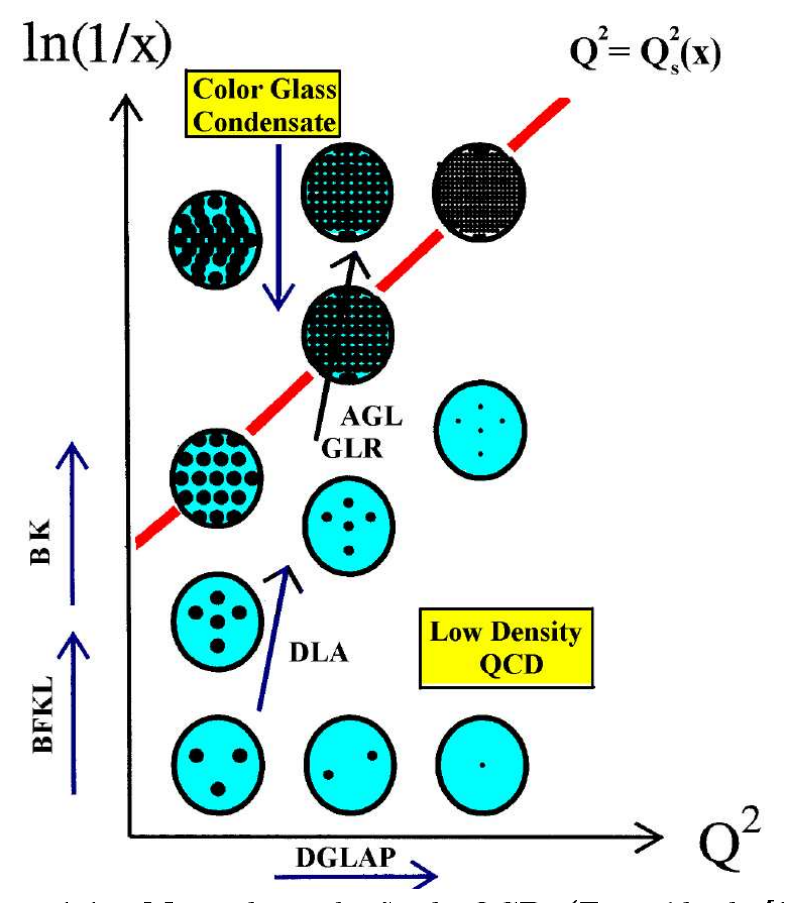

Figura 1.10: Mapa da evolução da QCD. (Extraído de [19]).

Na figura 1.10 é apresentado um mapa simbólico das equações de evolução da QCD. Nele vemos uma representação pictórica de como ocorre a evolução com $Q^{2}$ na equação de DGLAP. Nesta equação de evolução, fixamos $x$ e evoluímos em $Q^{2}$. Dessa forma, conforme aumentamos a nossa resolução, o sistema se torna mais diluído, pois, apesar do número de glúons crescer, a área de cada um torna-se menor. Nesta figura vemos também uma imagem pictórica de como a equação de BFKL descreve a evolução do sistema com a energia. Na BFKL o momento transferido $\left(Q^{2}\right)$ é fixado e evoluímos as funções de distribuição em relação a $x$. Desse modo, conforme aumentamos a energia, o número de partons dentro do hádron aumenta, mas a área de cada um deles continua sempre a mesma, já que a resolução (que depende de $Q^{2}$ ) continua a mesma. Nesta visão é fácil perceber que a saturação ocorre assim que toda a área do hádron for ocupada pelo crescente número de glúons. Nesta figura também é mostrada a equação de Ayala, Gay e Levin (AGL), que é uma equação de evolução não-linear [23, 24].

A linha " $Q_{s}$ " que aparece na figura 1.10 separa o regime linear do não linear. $Q_{s}$ é chamada de escala de saturação, a qual pode ser entendida usando argumentos 


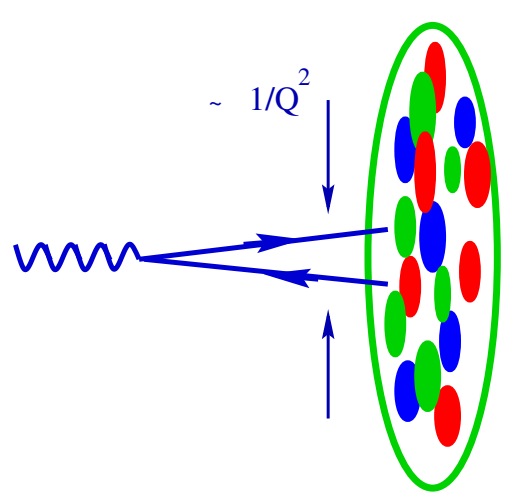

Figura 1.11: Resolução em termos da virtualidade $Q^{2}$. Fóton sondando o alvo.

geométricos [25]. Na figura 1.11 temos que um fóton com uma virtualidade $Q^{2}$ consegue resolver uma distância dentro do alvo da ordem de

$$
r \propto \frac{1}{\sqrt{Q^{2}}}
$$

A partir dessa relação vemos que, quando aumentamos $Q^{2}$ o dipolo torna-se mais compacto e assim consegue resolver objetos menores presentes no alvo (hádron), por exemplo, consegue "enxergar" os glúons. Consequentemente esse dipolo interage com um glúon de área transversal igual à sua. Assim, cada glúon irá ocupar uma área transversal da ordem de $\pi / Q^{2}$. E a seção de choque dipolo-glúon é aproximadamente

$$
\sigma \sim \alpha_{s}\left(Q^{2}\right) \frac{\pi}{Q^{2}}
$$

A área transversal do alvo (hádron) é igual a

$$
S_{A} \sim \pi R_{A}^{2}
$$

sendo $R_{A}$ o raio do alvo. Quando a soma de todas as áreas dos glúons for igual à área total do alvo, ou seja

$$
S_{A}=N_{A} \sigma \rightarrow N_{A} \sim \frac{S_{A}}{\sigma} \sim \frac{R_{A}^{2}}{\alpha_{s}} Q^{2}
$$


os glúons começam a sobrepor-se uns aos outros e o processo de recombinação de glúons torna-se relevante, inibindo o rápido crescimento da densidade de glúons. A partir da condição $(1,6.88)$ a escala de saturação fica definida como:

$$
Q_{s}^{2} \sim \alpha_{s} \frac{N_{A}}{R_{A}^{2}} \sim \frac{\alpha_{s}}{R_{A}^{2}} A x g\left(x, Q^{2}\right) \sim \alpha_{s} A^{1 / 3} x g\left(x, Q^{2}\right)
$$

Em 1999 alguns autores [26] passaram a afirmar que, no HERA, já estariam sendo observados alguns efeitos da saturação. A partir de 2000, com a entrada em operação do Relativistic Heavy Ion Collider (RHIC), no Brookhaven National Laboratory (BNL) a procura do CGC tornou-se ainda mais intensa. Surgiram inúmeros trabalhos propondo novos observáveis que pudessem revelar esta componente de alta densidade da função de onda dos hadrons e núcleos. O CGC é, às vezes, chamado de "um novo estado da matéria". Em 2004, os resultados do RHIC obtidos em colisões dêuteron-ouro levaram muitas pessoas a acreditar que o CGC tinha sido inequivocamente observado. Houve até um anúncio oficial ("press release") do BNL divulgando a descoberta do novo estado da matéria. Mais tarde, a certeza desta descoberta foi questionada. No artigo de revisão de Larry McLerran [27] as seguintes evidências experimentais do CGC são mencionadas:

1. "Scaling"geométrico da seção de choque fóton-próton medida no HERA, caracterizado pela dependência na seção de choque na variável $\tau=r Q_{s}$.

2. Comportamento da função de estrutura $F_{2}$ medida em DIS no HERA.

3. Comportamento da razão entre as seções de choque difrativa e total medidas no HERA.

4. Comportamento da seção de choque de fotoprodução do méson $\rho$ medida no HERA.

5. Comportamento da multiplicidade de partículas carregadas medidas na região central no RHIC. 
6. Comportamento da distribuição de momento transversal de partículas com grande $p_{T}$ e grande rapidez em colisões d-Au no RHIC.

Uma análise cuidadosa da literatura mostra que todos estes fenômenos podem ser explicados sem lançar mão do CGC. No entanto, como foi argumentado em [27], o CGC é uma teoria robusta, baseada em primeiros princípios da QCD e fornece uma explicação global para fenômenos muito diferentes entre si. Podemos concluir que a situação permanece em aberto e que a busca pela "assinatura"do CGC vai continuar. Neste sentido é interessante observar as evidências enumeradas acima para criarmos uma intuição de como o CGC modifica as previsões baseadas apenas na dinâmica linear da QCD. Por exemplo, na figura 1.12 vemos que as previsões do CGC diferem das feitas com o uso da solução da equação de BFKL na região de pequeno $x$ e pequeno $Q^{2}$, fazendo com que $F_{2}$ cresça muito mais suavemente do que a previsão da dinâmica linear. Vemos que quando os efeitos associados ao CGC são aplicados, ele funciona como um atenuador de tendências que já existem, ou seja, ele afeta os observáveis apenas quantitativamente.

$R_{C P}$ é a razão entre os espectros de momento transversal (de partículas carregadas) medidos no RHIC em colisões dêuteron-ouro centrais e periféricas e está mostrada na figura 1.13. Estes dados da colaboração BRAHMS mostram uma supressão do pico Cronin para valores crescentes da pseudorapidez.

Nesta região de momento transversal, uma grande parte da produção de partículas ocorre através do espalhamento gluon-gluon "hard"(ou "semihard") descrito pela QCD perturbativa. As colisões periféricas podem ser consideradas como colisões prótonpróton. O efeito Cronin ocorre quando o parton do dêuteron sofre reespalhamento antes da produção final de partículas observadas. Estas colisões múltiplas aumentam o $p_{T}$ do jato e das partículas observadas.

A normalização é tal que $R_{C P}=1$ significa que as colisões centrais são simples superposições de colisões próton-próton. Assim, quando observamos $R_{C P}>1$, estamos vendo o chamado "pico Cronin", que é uma indicação de reespalhamento, efeito já muitas vezes observado em colisões a energias menores, onde não se espera que o CGC 


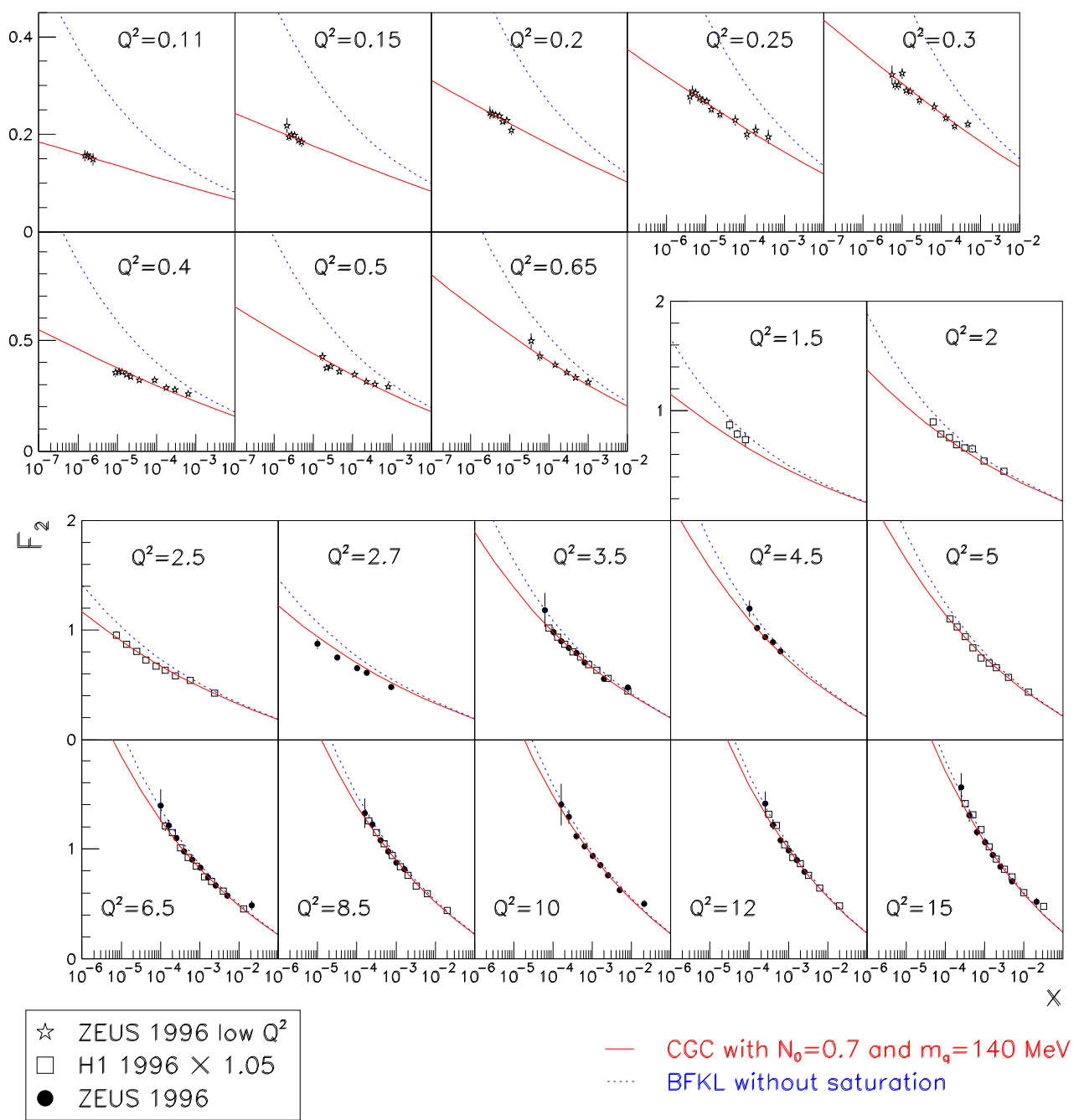

Figura 1.12: As funções de estrutura $F_{2}$ (Extraído de [50]).

tenha nenhuma importância.

Quando a produção de uma partícula ocorre através da fusão de dois glúons com momentos fracionários $y$ (vindo do dêuteron) e $x$ (vindo do ouro), sua pseudorapidez será dada por:

$$
\eta=\frac{1}{2} \ln \frac{y}{x}
$$

Quando medimos partículas com valores grandes de $\eta$ sabemos que $y \simeq 1$ e $x<<1$ e podemos afirmar que estamos "provando"a região de pequeno $x$ do alvo e entrando no domínio da saturação. Os dados mostram que há uma supressão pronunciada do pico 


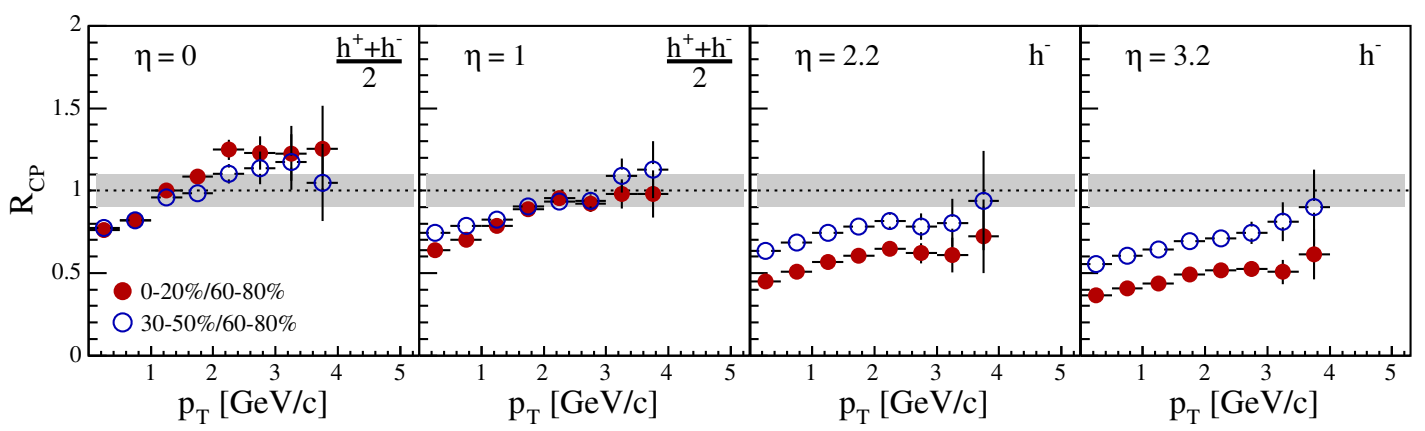

Figura 1.13: A razão $R_{C P}$ central para periférica de hádrons carregados e negativos como função de $p_{T}$ (Extraído de [25]).

Cronin com o aumento da rapidez. Esta supressão pode ser naturalmente entendida como um efeito da saturação, pois conforme diminuímos $x$, o número de glúons do alvo diminui (quando comparado ao caso não saturado) e assim ocorre uma diminuição do número de partículas produzidas.

Esta evidência experimental favorável ao CGC precisa ser confirmada através da análise de outros observáveis.

\subsection{Difração}

Outro tema de interesse nessa tese são os processo difrativos, os quais são um subconjunto das interações fóton-hádron e hádron-hádron, que do ponto de vista experimental são caracterizados pela existência de uma região do espaço de fase na qual não é observada nenhuma partícula. Esta região é chamada de "gap" de rapidez. Em 1987, Dokshitzer, Troyan e Khoze (e mais tarde Bjorken em 1992) propuseram usar a ocorrência de um grande gap de rapidez como assinatura de processos hadrônicos difrativos.

Do ponto de vista teórico dizemos que um processo é difrativo quando entre o projétil e o alvo ocorre a troca de um objeto com os números quânticos do vácuo. $\mathrm{Na}$ teoria de Regge este objeto é chamado de Pomeron. Desde o início dos anos oitenta surgiram várias tentativas de entender o Pomeron no contexto da QCD. Uma destas tentativas foi apresentada por Low e Nussinov. Nela o Pomeron é formado por dois 
glúons num estado singleto de cor. Este modelo de troca de dois glúons continua a ser utilizado.

O conceito de difração dura data de 1985, quando Ingelman e Schlein [28] sugeriram investigar a produção difrativa de jatos de alto $p_{T}$ como uma maneira de provar a estrutura partônica do Pomeron. A difração dura foi observada pela primeira vez no estudo da produção de jatos (separados por um gap de rapidez) feito pela colaboração UA8 em 1988. Nos anos seguintes, com os experimentos no HERA e no Tevatron, esta área entrou em sua fase madura, na qual surgiram seus principais resultados.

Uma fração significativa (em torno de 10\%) dos eventos de espalhamento inelástico profundo observados pelas colaborações ZEUS e H1 no HERA, são de natureza difrativa. Nestes eventos o próton que entra permanece intacto apesar da inelasticidade da reação, perdendo apenas uma pequena fração $x_{\mathbb{P}}$ de seu momento inicial. O estado final do próton está bem separado em rapidez do resto do sistema, o qual parece de um evento de DIS típico. Assim, um grande gap de rapidez serve realmente como uma assinatura para o DIS difrativo. Em linguagem partônica, um aglomerado de partons neutro de cor fragmenta-se independentemente do próton espalhado.

Alguns resultados experimentais para difração estão nas figuras 1.14 e 1.15 . Na figura 1.14 são mostrados os dados experimentais para a função de estrutura difrativa do próton $\left(F_{2}^{D(3)}\right)$ em função de $x_{\mathbb{P}}$ para valores fixos de $\beta$ e $Q^{2}$. Os dados indicam que $F_{2}^{D(3)}$ cresce para valores menores de $x_{\mathbb{P}}$. Na figura 1.15 é mostrada a razão entre as seções de choque difrativa e total. Os dados indicam que essa razão é independente da energia. As curvas apresentada nas figuras 1.14 e 1.15 são previsões de modelos que fazem uso da física de saturação. As variáveis $x_{\mathbb{P}}$ e $\beta$ estão definidas no capítulo 2 . 


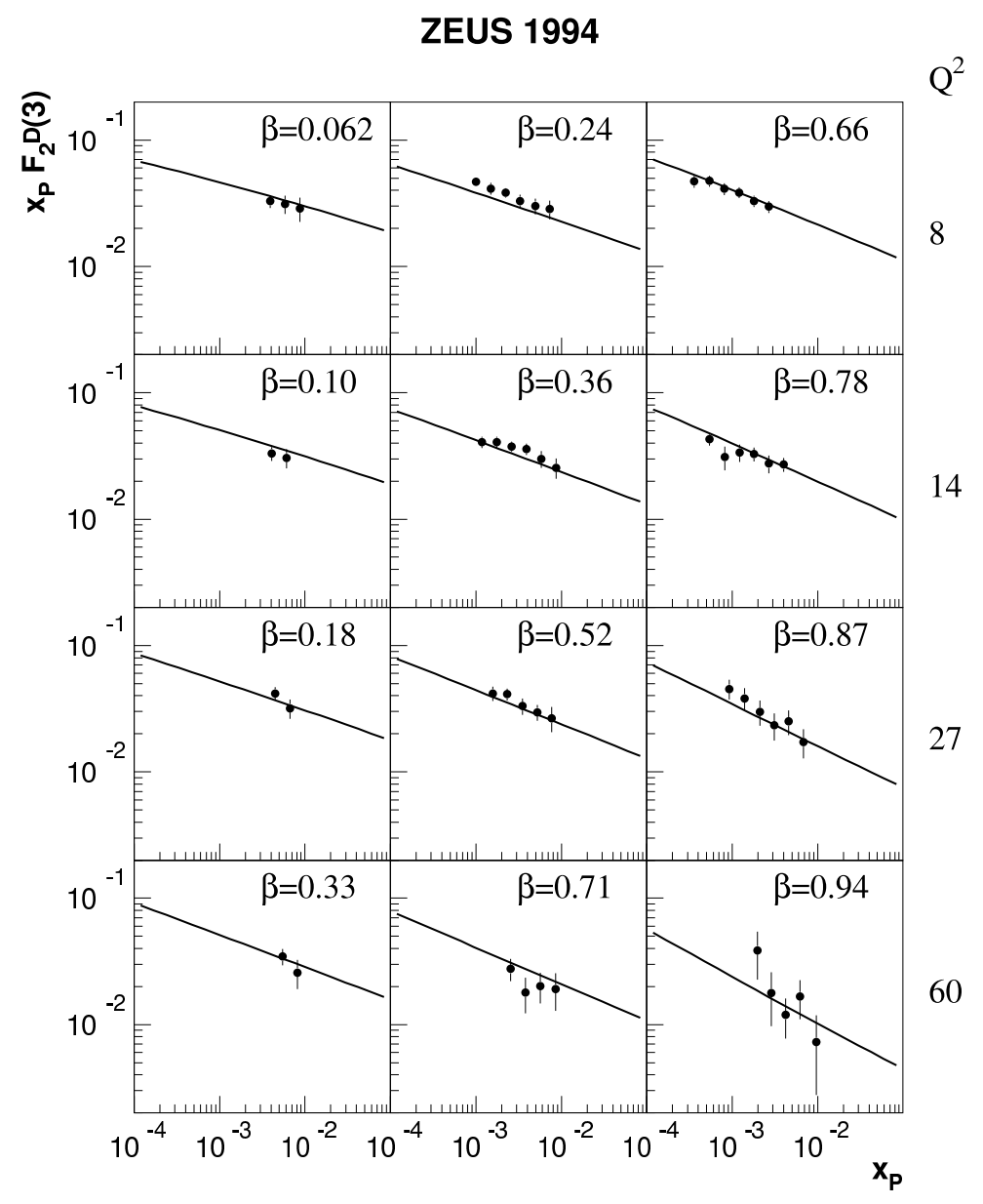

Figura 1.14: Função de estrutura difrativa (Extraído de [26]). 


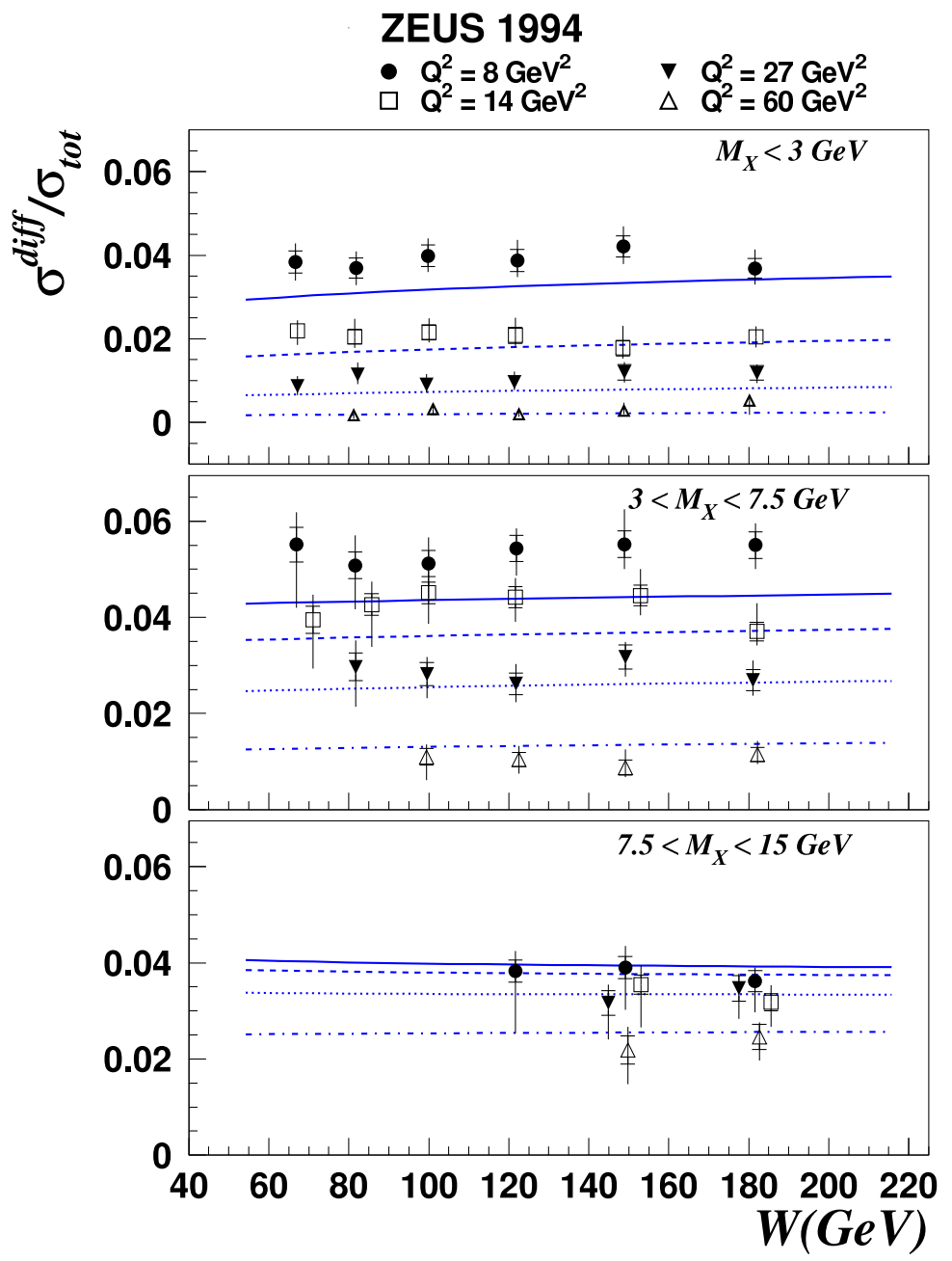

Figura 1.15: Razão $\sigma^{D} / \sigma^{\text {tot }}$ medida pela Colaboração ZEUS (Extraído de [26]). 


\section{Capítulo 2}

\section{Dipolos de cor}

\subsection{DIS na representação de dipolos de cor}

O formalismo de dipolo de cor é útil para o estudo do DIS no regime de $x$ pequeno, especialmente no referencial de repouso do próton. Neste referencial, quando $x \rightarrow 0$, o fóton virtual transforma-se em um par quark-antiquark e depois de um tempo longo ele colide com o alvo. Desde que o tempo de interação seja muito mais curto do que o tempo de vida do par, o tamanho transversal do par $q \bar{q}$ pode ser considerado fixo durante o processo de espalhamento [29].

O momento do fóton é:

$$
q=\left(\nu, 0,0, \sqrt{\nu^{2}+Q^{2}}\right)
$$

onde $\nu=k-k^{\prime}$ e $Q^{2}=-q^{2}$. Usando as variáveis do cone de luz escrevemos os momentos do fóton virtual $\left(\gamma^{*}\right)$, do quark $(q)$ e do anti-quark $(\bar{q})$ como:

$$
\begin{aligned}
& \gamma^{*}: \quad=\left(q^{+},-\frac{Q^{2}}{2 q^{+}}, \overrightarrow{0}\right) ; \\
& q: \quad k=\left(z q^{+}, \frac{k^{2}}{2 z q^{+}}, \vec{k}\right) ;
\end{aligned}
$$




$$
\bar{q}: \quad k^{\prime}=\left((1-z) q^{+}, \frac{k^{2}}{2(1-z) q^{+}},-\vec{k}\right)
$$

onde $z$ é a fração de momento do fóton carregada pelo quark e $(1-z)$ é a fração de momento do fóton carregada pelo anti-quark. A massa invariante quadrada do par $q \bar{q}$ é:

$$
M^{2}=\left(k+k^{\prime}\right)^{2}=\frac{\vec{k}^{2}}{z(1-z)}
$$

Usando o princípio da incerteza podemos estimar o tempo de vida do par $\tau_{f}$ :

$$
\tau_{f} \sim \frac{1}{\Delta E}
$$

onde $\Delta E=E_{\text {par }}-E_{\gamma^{*}}$ sendo

$$
E_{p a r}=\frac{1}{\sqrt{2}}\left(q^{+}+\frac{k^{2}}{2 z(1-z) q^{+}}\right)
$$

$\mathrm{e}$

$$
E_{\gamma^{*}}=\frac{1}{\sqrt{2}}\left(q^{+}-\frac{Q^{2}}{2 q^{+}}\right)
$$

Assim

$$
\Delta E=\frac{1}{2 \sqrt{2} q^{+}}\left(Q^{2}+\frac{k^{2}}{z(1-z)}\right)
$$

e considerando que $Q^{2} \gg M^{2}$ temos:

$$
\Delta E \simeq \frac{Q^{2}}{\sqrt{2} q^{+}}=m_{N} x
$$

Finalmente concluímos que:

$$
\tau_{f} \sim \frac{1}{\Delta E} \sim \frac{1}{m_{N} x}
$$

Vemos então que, quando $x \rightarrow 0, \tau_{f}$ é muito maior que o tempo típico de interação: $\tau_{\text {int }} \sim R_{p}$, onde $R_{p}$ é o raio do alvo, neste caso o próton. Consequentemente, podemos interpretar o DIS em $x$ pequeno como o espalhamento de um dipolo de cor $q \bar{q}$ com o nucleon. Isto nos leva a pensar que a descrição da seção de choque pode ser fatorizada 
da seguinte maneira

$$
\sigma^{\gamma^{*} p}=\int d z \int d r^{2}|\Psi|^{2} \sigma_{d i p}
$$

onde $r$ é o raio tranversal do par $q \bar{q}, \Psi$ é a sua função de onda que descreve a transição fóton virtual-dipolo, e $\sigma_{d i p}$ é a seção de choque de interação entre o par e o alvo. Nikolaev e Zakharov e posteriormente Mueller [30] demonstraram que esta expressão é de fato válida. Na próxima seção apresentamos de maneira resumida os argumentos que levam a esta conclusão.

\subsubsection{A fatorização $k_{T}$ e a seção de choque de dipolo}

Em altas energias $(s \rightarrow \infty$, ou seja, $x \rightarrow 0)$, a fatorização colinear deve ser generalizada, de forma a permitir que o parton incidente possua momento transversal não-nulo. É possível deduzir uma nova e mais geral fórmula de fatorização, chamada de "fatorização $k_{T}$ ". Com esta nova fórmula podemos escrever a contribuição do processo $\gamma^{*} g \rightarrow q \bar{q}$ para a seção de choque total $\gamma^{*} p$ da seguinte forma [29]:

$$
\sigma_{\lambda}^{\gamma^{*} p}\left(x, Q^{2}\right)=\int \frac{d \vec{K}^{2}}{\vec{K}^{2}} \int_{x}^{1} \frac{d x^{\prime}}{x^{\prime}} f\left(\frac{x}{x^{\prime}}, \vec{K}^{2}\right) \hat{\sigma}_{\lambda}^{\gamma^{*} g}\left(x^{\prime}, \vec{K}^{2}, Q^{2}\right)
$$

onde $\lambda$ denota a polarização do fóton virtual, $\hat{\sigma}_{\lambda}^{\gamma^{*} g}$ é a seção de choque gluônica e $f\left(\frac{x}{x^{\prime}}, \vec{K}^{2}\right)$ é a distribuição de glúons não integrada, a qual está relacionada com a distribuição usual de glúons da seguinte forma:

$$
x g\left(x, Q^{2}\right)=\int^{Q^{2}} \frac{d \vec{K}^{2}}{\vec{K}^{2}} f\left(x, \vec{K}^{2}\right) .
$$

A seção de choque do processo elementar

$$
\gamma^{*}(q)+g(K) \rightarrow q\left(k_{1}\right)+\bar{q}\left(k_{2}\right)
$$


é dada por [29]:

$$
\sigma_{\lambda}^{\gamma^{*} g}=\frac{\beta}{8 \pi^{2} W^{4} \vec{K}^{2}} \int \frac{d z}{z(1-z)} \int d^{2} \vec{k} \cdot \delta\left(\beta-\frac{z \vec{k}^{2}+(1-z)(\vec{k}+\vec{K})^{2}+\varepsilon^{2}}{z(1-z) W^{2}}\right) H_{\lambda},
$$

onde

$$
\varepsilon^{2} \equiv Q^{2} z(1-z), \beta=x / x^{\prime}
$$

é a fração do momento longitudinal do próton carregada pelo glúon, $W$ é a energia de centro de massa do sistema fóton virtual - próton, e

$$
\begin{aligned}
H_{\lambda}= & 8 \pi^{2} \alpha_{s} \alpha_{e m} \frac{z(1-z)}{Q^{2}} W^{4} \\
& \cdot\left\{\frac{N_{\lambda}(k, k)}{\left(\vec{k}^{2}+\varepsilon^{2}\right)^{2}}+\frac{N_{\lambda}(\vec{k}+\vec{K}, \vec{k}+\vec{K})}{\left.\left[(\vec{k}+\vec{K})^{2}+\varepsilon^{2}\right)\right]^{2}}-\frac{2 N_{\lambda}(\vec{k}, \vec{k}+\vec{K})}{\left.\left(\vec{k}^{2}+\varepsilon^{2}\right)\left[(\vec{k}+\vec{K})^{2}+\varepsilon^{2}\right)\right]}\right\}
\end{aligned}
$$

com

$$
\begin{aligned}
& N_{L}\left(\vec{K}_{1}, \vec{K}_{2}\right)=4 z^{2}(1-z)^{2} Q^{4}, \\
& N_{T}\left(\vec{K}_{1}, \vec{K}_{2}\right)=Q^{2}\left[z^{2}+(1-z)^{2}\right] K_{1} \cdot K_{2} .
\end{aligned}
$$

Com a expressão acima a seção de choque $\gamma^{*} p$ pode ser reescrita como:

$$
\sigma_{L, T}^{\gamma^{*} p}\left(x, Q^{2}\right)=\frac{\alpha_{e m}}{Q^{2}} \sum_{q} e_{q}^{2} \int \frac{d \vec{K}^{2}}{\vec{K}^{4}} \int_{0}^{1} d z \int d^{2} \vec{k} \alpha_{s}\left(\mu^{2}\right) f\left(\beta, \vec{K}^{2}\right) H_{\lambda}
$$

A seguir vamos inserir nas expressões acima as seguintes identidades:

$$
\begin{aligned}
\int \frac{d^{2} \vec{k}}{\left(\overrightarrow{k^{2}}+\varepsilon^{2}\right)\left[(\vec{k}+\vec{K})^{2}+\varepsilon^{2}\right]}= & \frac{1}{(2 \pi)^{2}} \int d^{2} \overrightarrow{k_{1}} \int d^{2} \overrightarrow{k_{2}} \int d^{2} \vec{r} \\
& \times \frac{e^{i \vec{r} \cdot\left(\overrightarrow{k_{1}}+\vec{K}\right)-i \vec{r} \cdot \overrightarrow{k_{2}}}}{\left({\overrightarrow{k_{1}}}^{2}+\varepsilon^{2}\right)\left(\vec{k}_{2}^{2}+\varepsilon^{2}\right)} \\
= & \int d^{2} \vec{r} e^{i \vec{r} \cdot \vec{K}}\left|\frac{1}{2 \pi} \int d^{2} \vec{k} \frac{e^{i \vec{k} \cdot \vec{r}}}{\vec{k}^{2}+\varepsilon^{2}}\right|^{2}
\end{aligned}
$$


$\mathrm{e}$

$$
\begin{aligned}
\int d^{2} \vec{k} \frac{\vec{k}^{2}+\vec{k} \cdot \vec{K}}{\left.\left(\vec{k}^{2}+\varepsilon^{2}\right)\left[(\vec{k}+\vec{K})^{2}+\varepsilon^{2}\right)\right]}= & \frac{1}{(2 \pi)^{2}} \int d^{2} \overrightarrow{k_{1}} \int d^{2} \overrightarrow{k_{2}} \int d^{2} \vec{r} \\
& \times \frac{\left(\vec{\nabla} e^{i \vec{r} \cdot\left(\overrightarrow{k_{1}}+\vec{K}\right)}\right) \cdot\left(\vec{\nabla} e^{-i \vec{r} \cdot \overrightarrow{k_{2}}}\right)}{\left(\vec{k}_{1}^{2}+\varepsilon^{2}\right)\left(\vec{k}_{2}^{2}+\varepsilon^{2}\right)}
\end{aligned}
$$

onde $\vec{\nabla} \equiv \partial / \partial \vec{r}$. Com estas identidades passamos do espaço dos momentos transversais para o espaço das coordenadas transversais, onde a variável $r$ será identificada com a separação transversal do dipolo $q \bar{q}$. Após integrações angulares (que fazem aparecer as funções de Bessel) e um reagrupamento de termos, chegamos a:

$$
\sigma_{L, T}^{\gamma^{*} p}\left(x, Q^{2}\right)=\int_{0}^{1} d z \int d^{2} \vec{r}\left|\Psi_{L, T}(z, r)\right|^{2} \sigma_{d i p}(x, r)
$$

onde

$$
\begin{aligned}
\left|\Psi_{L}(z, r)\right|^{2} & =\frac{6 \alpha_{e m}}{(2 \pi)^{2}} \sum_{q} 4 e_{q}^{2} Q^{2} z^{2}(1-z)^{2} K_{0}^{2}(\varepsilon r) \\
\left|\Psi_{T}(z, r)\right|^{2} & =\frac{6 \alpha_{e m}}{(2 \pi)^{2}} \sum_{q} e_{q}^{2}\left[z^{2}+(1-z)^{2}\right] \varepsilon^{2} K_{1}^{2}(\varepsilon r)
\end{aligned}
$$

$\mathrm{e}$

$$
\sigma_{d i p}(x, r)=\frac{4 \pi}{3} \int \frac{d^{2} \vec{K}}{\vec{K}^{4}} \alpha_{s} f\left(x, \vec{K}^{2}\right)\left(1-e^{i \vec{K} \cdot \vec{r}}\right),
$$

onde $L$ e $T$ significam respectivamente polarização longitudinal e transversal do fóton.

A relação $(2,1.10)$ é independente de referencial. Podemos interpretar $\left|\Psi_{L, T}(z, r)\right|^{2}$ como sendo a probabilidade do fóton virtual flutuar em um par quark-antiquark e depois interagir com o alvo. Esta interação está representada pela quantidade $\sigma_{d i p}(x, r)$. Por sua vez, $\sigma_{d i p}(x, r)$, está relacionada com a distribuição de gluons não integrada e consequentemente com a dinâmica.

Uma importante relação entre a seção de choque de dipolo e a densidade de glúons 
é obtida fazendo a integral angula e reescrevendo $\sigma_{d i p}(x, r)$ como:

$$
\sigma_{d i p}(x, r)=\frac{\pi}{3} r^{2} \int_{0}^{\infty} \frac{d \vec{K}^{2}}{\vec{K}^{2}} \alpha_{s} \frac{4\left[1-J_{0}(K r)\right]}{(K r)^{2}} \frac{\partial x g\left(x, \vec{K}^{2}\right)}{\partial \ln \vec{K}^{2}}
$$

A função $4\left[1-J_{0}(\xi)\right] / \xi^{2}$ pode ser aproximada pela função $\Theta\left(A-\ln \xi^{2}\right) \operatorname{com} A \simeq 10 \mathrm{e}$ assim:

$$
\sigma_{d i p}(x, r)=\frac{\pi}{3} r^{2} \alpha_{s}(r) x g\left(x, A / r^{2}\right)
$$

Esta relação é válida na aproximação de duplo logarítmo dominante (DLLA) e no regime linear da dinâmica das interações fortes. Além disso, ela demonstra que: $\sigma_{\text {dip }} \propto$ $r^{2}$, o que implica que a interação se anula para $r \rightarrow 0$. Esta propriedade denomina-se transparência de cor.

Antes de estudarmos as propriedades de $\sigma(x, r)$ é útil mostrarmos como $\sigma(x, r)$ aparece também no caso da seção de choque difrativa.

Seja $T$ a matriz de transição que descreve o espalhamento elástico e difrativo de um hádron $N$. Supondo que a amplitude de espalhamento seja puramente imaginária: $T=i \mathcal{D}$, onde $\mathcal{D}$ é real. Vamos considerar uma base de estados hadrônicos físicos $|i\rangle$. O espalhamento difrativo leva um desses estados em outro e o espalhamento elástico leva cada estado nele mesmo. Assim $\mathcal{D}_{i k} \equiv\langle k|\mathcal{D}| i\rangle$ é a amplitude para a transição difrativa, $|k\rangle \rightarrow|i\rangle$. Os elementos da diagonal $\mathcal{D}_{i i} \equiv\langle i|\mathcal{D}| i\rangle$ são as amplitudes elásticas.

Introduzimos um conjunto completo de auto-estados de $\mathcal{D}$ :

$$
\mathcal{D}|\alpha\rangle=d_{\alpha}|\alpha\rangle
$$

O auto-valor $d_{\alpha}$ é proporcional à seção de choque total para o espalhamento $\alpha N$, que chamamos $\sigma_{\alpha}$ :

$$
\begin{aligned}
\sigma_{\alpha} & \equiv \sigma_{\text {tot }}^{\alpha N}=\frac{1}{s} \operatorname{Im}\langle\alpha|i \mathcal{D}| \alpha\rangle \\
& =\frac{1}{s} \operatorname{Im}\left(i\langle\alpha \mid \alpha\rangle d_{\alpha}\right)=\frac{1}{s} d_{\alpha} .
\end{aligned}
$$


Podemos expandir o estado físico $|i\rangle$ em termos de $|\alpha\rangle$ :

$$
|i\rangle=\sum_{\alpha} c_{i \alpha}|\alpha\rangle
$$

Os elementos de matriz do operador $\mathcal{D}$ são:

$$
\begin{aligned}
\mathcal{D}_{i k} & =\langle k|\mathcal{D}| i\rangle \\
& =\sum_{\alpha} \sum_{\beta} c_{k \beta}^{*} c_{i \alpha}\langle\beta|\mathcal{D}| \alpha\rangle \\
& =\sum_{\alpha} \sum_{\beta} c_{k \beta}^{*} c_{i \alpha}\langle\beta \mid \alpha\rangle d_{\alpha} \\
& =\sum_{\alpha} c_{k \alpha}^{*} c_{i \alpha} d_{\alpha} .
\end{aligned}
$$

A amplitude de espalhamento elástico fica:

$$
\begin{aligned}
\mathcal{D}_{i i} & =\langle i|\mathcal{D}| i\rangle \\
& =\sum_{\alpha} c_{i \alpha}^{*} c_{i \alpha} d_{\alpha} \\
& =\sum_{\alpha}\left|c_{i \alpha}\right|^{2} d_{\alpha}
\end{aligned}
$$

e a seção de choque total do espalhamento $i N$ fica:

$$
\begin{aligned}
\sigma_{\text {tot }}^{i N} & =\frac{1}{s} \mathcal{D}_{i i} \\
& =\sum_{\alpha}\left|c_{i \alpha}\right|^{2} \frac{d_{\alpha}}{s} \\
& =\sum_{\alpha}\left|c_{i \alpha}\right|^{2} \sigma_{\alpha} .
\end{aligned}
$$


O valor esperado do operador $\mathcal{O}$ no estado $|i\rangle$ é:

$$
\begin{aligned}
\langle\mathcal{O}\rangle & \equiv\langle i|\mathcal{O}| i\rangle \\
& =\sum_{\alpha \beta}\langle i \mid \alpha\rangle\langle\alpha|\mathcal{O}|\rangle \beta\langle\beta \mid i\rangle \\
& =\sum_{\alpha \beta} c_{i \alpha} c_{i \beta}^{*}\langle\alpha|\mathcal{O}| \beta\rangle .
\end{aligned}
$$

Se $\mathcal{O}$ é diagonal na base $|\alpha\rangle$, então:

$$
\langle\mathcal{O}\rangle=\sum_{\alpha}\left|c_{i \alpha}\right|^{2} \mathcal{O}_{\alpha}
$$

onde $\mathcal{O}_{\alpha} \equiv\langle\alpha|\mathcal{O}| \alpha\rangle$. Assim temos que:

$$
\sigma_{t o t}^{i N}=\left\langle\sigma_{\alpha}\right\rangle
$$

Pela definição, a seção de choque difrativa em $t=0$ é:

$$
\begin{aligned}
\left.\frac{d \sigma_{i N}^{D}}{d t}\right|_{t=0} & =\frac{1}{16 \pi s^{2}} \sum_{k \neq i} \mathcal{D}_{i k}^{2} \\
& =\frac{1}{16 \pi s^{2}}\left(\sum_{k} \mathcal{D}_{i k}^{2}-\mathcal{D}_{i i}^{2}\right),
\end{aligned}
$$

usando a completeza do estado $|k\rangle$, temos

$$
\left.\frac{d \sigma_{i N}^{D}}{d t}\right|_{t=0}=\frac{1}{16 \pi s^{2}}\left(\left\langle i\left|\mathcal{D}^{2}\right| i\right\rangle-\langle i|\mathcal{D}| i\rangle^{2}\right)
$$

Expandindo $|i\rangle$ como: $|i\rangle=\sum_{\alpha} c_{i \alpha}|\alpha\rangle$, teremos:

$$
\left.\frac{d \sigma_{i N}^{D}}{d t}\right|_{t=0}=\frac{1}{16 \pi}\left(\left\langle\sigma_{\alpha}^{2}\right\rangle-\left\langle\sigma_{\alpha}\right\rangle^{2}\right)
$$


onde fizemos:

$$
\begin{aligned}
D_{i k} & =\langle k|D| i\rangle \\
\sum_{k} D_{i k}^{2} & =\sum_{k}\langle k|D| i\rangle^{*}\langle k|D| i\rangle=\sum_{k}\langle i|D| k\rangle\langle k|D| i\rangle=\left\langle i\left|D^{2}\right| i\right\rangle
\end{aligned}
$$

Agora vamos usar o formalismo descrito acima no caso do espalhamento inelástico profundo difrativo (DDIS) [29].

No DDIS, os auto-estados difrativos são os dipolos de cor $q \bar{q}$. Assim o estado $|\alpha\rangle$ definido acima será identificado com estado de Fock $q \bar{q}$ (o estado no qual o fóton virtual, $\gamma^{*}$, flutua antes de encontrar o alvo). A seção de choque $\sigma_{\alpha}$ será substituída pela seção de choque de espalhamento dipolo-próton $\sigma_{d i p}(x, r)$. Assim podemos escrever:

$$
\left.\frac{d \sigma_{L, T}^{D}}{d t}\right|_{t=0}=\frac{1}{16 \pi}\left(\left\langle\sigma_{d i p}^{2}(x, r)\right\rangle_{L, T}-\left\langle\sigma_{d i p}(x, r)\right\rangle_{L, T}^{2}\right)
$$

onde

$$
\left\langle\sigma_{d i p}(x, r)\right\rangle_{L, T} \equiv \int_{0}^{1} d z \int d^{2} \vec{r}\left|\Psi_{L, T}(z, r)\right|^{2} \sigma_{d i p}(x, r)
$$

Uma vez que $\left\langle\sigma_{d i p}(x, r)\right\rangle_{L, T} \equiv \sigma_{L, T}^{\gamma^{*} p}=\mathcal{O}\left(\alpha_{e m}\right)$, podemos desprezar o termo $\left\langle\sigma_{d i p}(x, r)\right\rangle^{2}$, e assim obtemos

$$
\left.\frac{d \sigma_{L, T}^{D}}{d t}\right|_{t=0}=\frac{1}{16 \pi}\left\langle\sigma_{d i p}^{2}(x, r)\right\rangle_{L, T}=\frac{1}{16 \pi} \int_{0}^{1} d z \int d^{2} \vec{r}\left|\Psi_{L, T}(z, r)\right|^{2} \sigma_{d i p}^{2}(x, r)
$$

A seção de choque difrativa é finalmente dada por

$$
\begin{aligned}
\sigma_{d i f f} & =\int_{-\infty}^{0} d t \frac{d \sigma}{d t}=\left.\int_{-\infty}^{0} d t e^{-B_{D} t} \frac{d \sigma}{d t}\right|_{t=0} \\
& =\frac{1}{16 \pi B_{D}} \int d z \int d^{2} r\left|\Psi_{L, T}(z, r)\right|^{2} \sigma_{d i p}^{2}(x, r)
\end{aligned}
$$




\subsubsection{Análise qualitativa}

Com algumas aproximações simples podemos estudar analiticamente as principais propriedades das seções de choque total e difrativa. No limite em que $r \rightarrow 0$ observamos que

$$
\sigma_{d i p}(x, r) \sim r^{2}
$$

de forma que pares de pequenos tamanhos interagem pouco com o próton. Essa propriedade é chamada de transparência de cor. Um cálculo mais preciso [29] de $\sigma(x, r)$ em $r$ pequeno dá:

$$
\sigma_{d i p}(x, r) \sim r^{2} \alpha_{s}(r) \ln \left(\ln \frac{r^{2}}{r_{0}^{2}}\right)
$$

que, quando substituída em $(2,1.10)$, leva à seguinte $\sigma_{T}^{\gamma^{*} p}$ :

$$
\sigma_{T}^{\gamma^{*} p} \sim \frac{1}{Q^{2}} \ln ^{2}\left(\ln \frac{Q^{2}}{\Lambda^{2}}\right)
$$

Para grandes $r$, devido ao confinamento, $\sigma_{\text {dip }}(x, r)$ satura em algum valor típico de seção de choque hádron-nucleon $\sigma_{0}$ :

$$
\sigma(x, r)_{d i p} \sim \sigma_{0}
$$

Para fótons transversais, a integral de normalização $\int d z \int d^{2} \vec{r}\left|\Psi_{T}(z, r)\right|^{2}$ diverge logaritmicamente no limite ultravioleta, ou seja, no limite de pequenas distâncias. Neste limite temos que $K_{1}(y) \sim 1 / y$ quando $y \rightarrow 0$. Isto não é problema, uma vez que $\sigma(x, r) \sim r^{2}$ para pequenos raios e as seções de choque físicas são finitas.

Como $K_{0}(y)$ e $K_{1}(y)$ caem exponencialmente em grande $y$, a contribuição dominante para $\sigma_{L, T}^{\gamma^{*} p}$ vem dos pares $q \bar{q}$ de tamanho:

$$
r^{2} \sim \frac{1}{\varepsilon^{2}}=\frac{1}{Q^{2} z(1-z)}
$$


Assim os pares assimétricos (pares com $z \approx 0$ ou $z \approx 1$ ), nos quais um dos pártons carrega a maior parte do momento (é chamada de configuração aligned-jet), têm grande tamanho $r \geq R \gg 1 / Q$, onde $R \sim 1 \mathrm{fm}$ é o raio de confinamento típico. Esta configuração é chamado de "jato alinhado".

Já os pares simétricos (pares com $z \approx 1 / 2$ ), onde quark e anti-quark carregam uma fração semelhante de momento longitudinal, têm tamanhos menores $r \leq 1 / Q$.

Consideremos a configuração simétrica, $z \sim 1 / 2$ e $r \sim 1 / Q$. Devido ao comportamento exponencial das funções de Bessel modificadas $K_{0}$ e $K_{1}$ introduzimos um corte na integral em $r$. Podemos fazer uma estimativa de $\sigma_{L, T}^{\gamma^{*} p}$, aproximando $K_{0}$ e $K_{1}$ pelas funções $\Theta$. Em particular, fazemos as aproximações $K_{0}(\varepsilon r) \sim \Theta(1-\varepsilon r)$ e $K_{1}(\varepsilon r) \sim \Theta(1-\varepsilon r) /(\varepsilon r)$. Assim

$$
\begin{aligned}
\sigma_{T}^{\gamma^{*} p} & \sim \int d z\left[z^{2}+(1-z)^{2}\right] \int_{0}^{1 / Q^{2}} d r^{2} \frac{\sigma(r)}{r^{2}} \\
& \sim \int d z\left[z^{2}+(1-z)^{2}\right] \frac{1}{Q^{2}} \sim \frac{1}{Q^{2}}
\end{aligned}
$$

e

$$
\begin{aligned}
\sigma_{L}^{\gamma^{*} p} & \sim Q^{2} \int d z\left[z^{2}(1-z)^{2}\right] \int_{0}^{1 / Q^{2}} d r^{2} \sigma(r) \\
& \sim Q^{2} \int d z\left[z^{2}(1-z)^{2}\right] \frac{1}{Q^{4}} \sim \frac{1}{Q^{2}}
\end{aligned}
$$

Vemos que para uma configuração simétrica, as seções de choque transversal e longitudinal têm a mesma dependência em $Q^{2}$.

Consideremos agora a configuração assimétrica $\left(z \sim \mu^{2} / Q^{2}\right.$ e $r \sim 1 / \mu$ onde $\mu \sim$ $1 / R)$. Assim

$$
\begin{aligned}
\sigma_{T}^{\gamma^{*} p} & \sim \int d z\left[z^{2}+(1-z)^{2}\right] \int_{0}^{1 / \mu^{2}} d r^{2} \frac{\sigma(r)}{r^{2}} \\
& \sim \int d z\left[z^{2}+(1-z)^{2}\right] \frac{1}{\mu^{2}} \sim \frac{\mu^{2}}{Q^{2}} \frac{1}{\mu^{2}} \sim \frac{1}{Q^{2}}
\end{aligned}
$$


$\mathrm{e}$

$$
\begin{aligned}
\sigma_{L}^{\gamma^{*} p} & \sim Q^{2} \int d z\left[z^{2}(1-z)^{2}\right] \int_{0}^{1 / \mu^{2}} d r^{2} \sigma(r) \\
& \sim Q^{2} \int d z\left[z^{2}(1-z)^{2}\right] \frac{1}{\mu^{4}} \sim Q^{2} \frac{\mu^{6}}{Q^{6}} \frac{1}{\mu^{4}} \sim \frac{\mu^{2}}{Q^{4}}
\end{aligned}
$$

Vemos que os pares grandes, têm comportamento diferente dos pequenos. Na configuração de jato alinhado $\sigma_{L}^{\gamma^{*} p}$ é suprimida por um fator $1 / Q^{2}$ com respeito a $\sigma_{T}^{\gamma^{*} p}$.

Podemos fazer este estudo qualitativo também para o espalhamento $\gamma^{*} p$ difrativo. Assim, no caso de pares assimétricos (dipolos grandes) temos:

$$
\begin{aligned}
\left.\frac{d \sigma_{T}^{D}}{d t}\right|_{t=0} & \sim \int d z\left[z^{2}+(1-z)^{2}\right] \int_{\frac{1}{\mu^{2}}}^{\infty} d r^{2} \varepsilon^{2} \frac{1}{\varepsilon^{2} r^{2}} \sigma^{2}(r) \\
& \sim \frac{\mu^{2}}{Q^{2}} \frac{1}{\mu^{4}}=\frac{1}{\mu^{2} Q^{2}}
\end{aligned}
$$

$\mathrm{e}$

$$
\begin{aligned}
\left.\frac{d \sigma_{L}^{D}}{d t}\right|_{t=0} & \sim Q^{2} \int d z z^{2}(1-z)^{2} \int_{\frac{1}{\mu^{2}}}^{\infty} d r^{2} \sigma^{2}(r) \\
& \sim Q^{2} \frac{\mu^{6}}{Q^{6}} \frac{1}{\mu^{6}}=\frac{1}{Q^{4}},
\end{aligned}
$$

onde $\mu \sim m_{q} \sim 1 / R$.

No caso dos pares simétricos (dipolos pequenos) temos:

$$
\begin{aligned}
\left.\frac{d \sigma_{T}^{D}}{d t}\right|_{t=0} & \sim \int d z\left[z^{2}+(1-z)^{2}\right] \int_{0}^{\frac{1}{Q^{2}}} d r^{2} \varepsilon^{2} \frac{1}{\varepsilon^{2} r^{2}} \sigma^{2}(r) \\
& \sim \frac{1}{Q^{4}}
\end{aligned}
$$


$\mathrm{e}$

$$
\begin{aligned}
\left.\frac{d \sigma_{L}^{D}}{d t}\right|_{t=0} & \sim Q^{2} \int d z z^{2}(1-z)^{2} \int_{0}^{\frac{1}{Q^{2}}} d r^{2} \sigma^{2}(r) \\
& \sim Q^{2} \frac{1}{Q^{6}}=\frac{1}{Q^{4}} .
\end{aligned}
$$

Em uma forma compacta escrevemos:

$$
\sigma_{L, T}^{\gamma^{*} p} \sim W_{L, T}^{\alpha} \sigma_{\alpha}
$$

e

$$
\left.\frac{d \sigma_{L}^{D}}{d t}\right|_{t=0} \sim W_{L, T}^{\alpha} \sigma_{\alpha}^{2}
$$

onde $\alpha$ denota o dipolo de cor e $W_{L, T}^{\alpha}$ são os pesos, os quais incorporam a função de onda do fóton e as integrações. As seções de choque de dipolo são quantidades universais e comportam-se como:

$$
\begin{aligned}
\sigma_{\alpha} & \sim \frac{1}{Q^{2}} \text { para pequenos dipolos } \\
\sigma_{\alpha} & \sim \frac{1}{\mu^{2}} \text { para grandes dipolos }
\end{aligned}
$$

As funções peso dependem da polarização do fóton virtual. Convencionalmente,

$$
\begin{aligned}
& W_{T}^{\alpha} \sim 1, W_{L}^{\alpha} \sim 1 \text { para pequenos dipolos } \\
& W_{T}^{\alpha} \sim \frac{\mu^{2}}{Q^{2}}, W_{L}^{\alpha} \sim \frac{\mu^{4}}{Q^{4}} \text { para grandes dipolos }
\end{aligned}
$$

A primeira conclusão que podemos obter desses resultados é que o DDIS é dominado por pares grandes e assimétricos, com a seção de choque de dipolo determinada pelo seu comportamento na região de grandes separações. Ao contrário, o DIS inclusivo tem contribuições iguais de pares pequenos e grandes. Embora, $Q^{2}$ seja uma escala dura, DDIS é amplamente não-perturbativo. Para podermos tratá-lo como um processo perturbativo temos que selecionar estados finais especiais: jatos com grande 


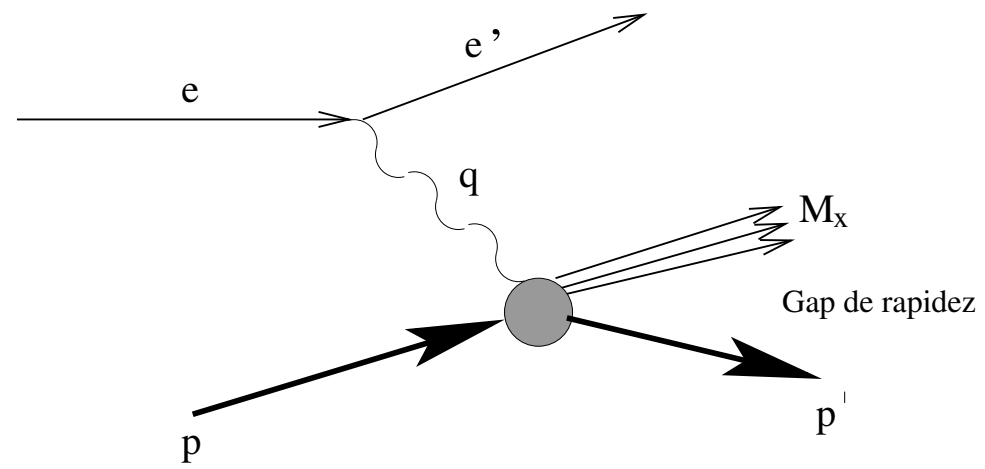

Figura 2.1: Espalhamento Inelástico Profundo Difrativo.

$p_{T}$, produção de quarks pesado, ou mésons vetoriais. Nestes processo o dipolo tem pequeno tamanho transversal e a teoria de perturbação é aplicável. No caso inclusivo, os fenômenos de curtas e longas distâncias coexistem. Podemos discriminar entre essas duas classes de contribuições, observando a dependência na energia da seção de choque difrativa, uma vez que a componente dura cresce mais rápido com a energia do que a suave.

\subsection{Espalhamento difrativo}

\subsubsection{A cinemática do DDIS}

No DDIS estudamos o seguinte processo: $l(l)+p(P) \rightarrow l^{\prime}\left(l^{\prime}\right)+p^{\prime}\left(P^{\prime}\right)+X\left(P_{X}\right)$, descrito na figura 2.1. As variáveis cinemáticas do DIS difrativo são [29]:

$$
\begin{gathered}
t=-\left(P^{\prime}-P\right)^{2} \simeq \frac{\vec{P}_{\perp}^{\prime}}{x_{F}} \\
x_{\mathbb{P}}=\frac{\left(P^{\prime}-P\right) \cdot q}{P \cdot q}=\frac{M_{X}^{2}+Q^{2}-t}{W^{2}+Q^{2}-m_{N}^{2}} \simeq \frac{M_{X}^{2}+Q^{2}}{W^{2}+Q^{2}}=1-x_{F} \\
\beta=\frac{Q^{2}}{2 q \cdot\left(P^{\prime}-P\right)}=\frac{Q^{2}}{M_{X}^{2}+Q^{2}-t} \simeq \frac{Q^{2}}{M_{X}^{2}+Q^{2}}
\end{gathered}
$$


Podemos relacionar $x, x_{\mathbb{P}}$ e $\beta$ da seguinte forma:

$$
x=\beta x_{\mathbb{P}}
$$

Além disso vemos que $M_{X}^{2}$ é a massa invariante do sistema $X, x_{F}$ é a variável de Feynman, $x_{\mathbb{P}}$ é a fração do momento longitudinal do próton carregada pelo Pomeron e $\beta$ é interpretado como a fração do momento do Pomeron carregada pelo párton que interage.

\subsubsection{Funções de estrutura difrativa}

No DIS difrativo é útil descrever os dados experimentais em termos das funções de estrutura difrativa. A seção de choque diferencial $e p$ com respeito às quatro variáveis define as funções de estrutura difrativa longitudinal e transversal e é dada por [29, 31, $32,33]:$

$$
\frac{d \sigma_{\gamma^{*} p}^{D}}{d x d Q^{2} d x_{\mathbb{P}} d t}=\frac{4 \pi \alpha_{e m}^{2}}{x Q^{4}}\left\{1-y+\frac{y^{2}}{2\left[1+R^{D(4)}\left(x, Q^{2}, x_{\mathbb{P}}, t\right)\right]}\right\} F_{2}^{D(4)}\left(x, Q^{2}, x_{\mathbb{P}}, t\right)
$$

onde $R^{D(4)}=F_{L}^{D(4)} / F_{T}^{D(4)}$. Supondo $F_{L}^{D(4)} \ll F_{T}^{D(4)}$ em $\beta \leq 0.8-0.9$ temos:

$$
\frac{d \sigma_{\gamma^{*} p}^{D}}{d x d Q^{2} d x_{\mathbb{P}} d t}=\frac{4 \pi \alpha_{e m}^{2}}{x Q^{4}}\left(1-y+\frac{y^{2}}{2}\right) F_{2}^{D(4)}\left(x, Q^{2}, x_{\mathbb{P}}, t\right)
$$

Quando o próton que sai não é detectado, não existe medida de "t" e apenas a seção de choque integrada em "t" é obtida:

$$
\frac{d \sigma_{\gamma^{*} p}^{D}}{d x d Q^{2} d x_{\mathbb{P}}}=\frac{4 \pi \alpha_{e m}^{2}}{x Q^{4}}\left(1-y+\frac{y^{2}}{2}\right) F_{2}^{D(3)}\left(x, Q^{2}, x_{\mathbb{P}}\right)
$$

onde

$$
F_{2}^{D(3)}\left(x, Q^{2}, x_{\mathbb{P}}\right)=\int_{0}^{\infty} d|t| F_{2}^{D(4)}\left(x, Q^{2}, x_{\mathbb{P}}, t\right)
$$

É usual estudarmos o comportamento da quantidade $x_{\mathbb{P}} F_{2}^{D(3)}$ com respeito às 


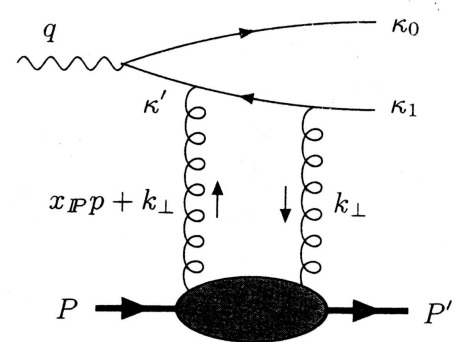

(a)

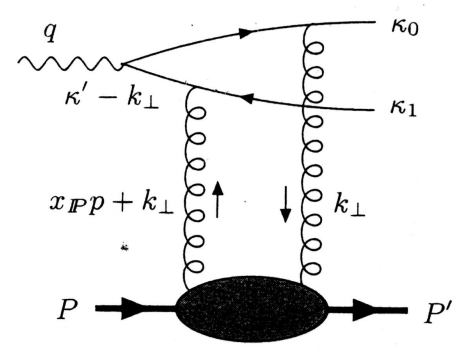

(b)

Figura 2.2: Produção difrativa do par $q \bar{q}$.

variáveis $x_{\mathbb{P}}$ e $\beta$. Faremos esse estudo no Capítulo 5 .

\subsubsection{O modelo de troca de dois gluons}

As funções de estrutura difrativas podem ser calculadas com o modelo de troca de dois gluons. Os cálculos foram feitos por vários autores em diversos trabalhos, a começar por Wüsthoff [34], depois por Golec-Biernat e Wüsthoff [35] e, mais recentemente, por Forshaw, Sandapen e Shaw [36], que escreveram as expressões das componentes de $F_{2}^{D(3)}$ na forma mais conveniente e que será utilizada por nós. A derivação destas fórmulas é bastante complicada e não será discutida aqui. Vamos, no que se segue, apresentar as fórmulas principais, comentá-las e reproduzir algumas passagens finais dos cálculos, onde surgem as fórmulas usadas nos próximos capítulos.

\section{A componente quark-antiquark transversal}

No formalismo de dipolos de cor a interação se dá entre o quark e o antiquark do dipolo com o alvo através da troca de dois gluons, como mostrado na figura 2.2.

A cinemática do processo é descrita em termos da decomposição de Sudakov dos 
momentos finais do quark e antiquark [29, 37]:

$$
\begin{aligned}
& k_{0}=(1-z) q^{\prime}+\frac{\vec{k}^{2}}{(1-z) W^{2}} P-k_{\perp} \\
& k_{1}=z q^{\prime}+\frac{\vec{k}^{2}}{z W^{2}} P+k_{\perp}
\end{aligned}
$$

onde $q^{\prime}=q+x P$ e onde usamos a condição de camada de massa para partículas sem massa. A massa produzida difrativamente é:

$$
M_{X}^{2}=\left(k_{0}+k_{1}\right)^{2}=\frac{\vec{k}^{2}}{z(1-z)} .
$$

O momento do quark denotado por $k^{\prime}$ no digrama 2.2 é:

$$
k^{\prime}=k_{1}-x_{\mathbb{P}} p=z q^{\prime}+\left(\frac{\vec{k}^{2}}{z W^{2}}-x_{\mathbb{P}}\right) P+k_{\perp}
$$

e o seu quadrado é:

$$
k^{\prime^{2}}=-\frac{\vec{k}^{2}}{(1-z)(1-\beta)}=-\frac{\vec{k}^{2}+z(1-z) Q^{2}}{1-z}
$$

onde usamos

$$
M_{X}^{2}=W^{2} x_{\mathbb{P}}(1-\beta) \text { e } \beta=Q^{2} /\left(Q^{2}+M_{X}^{2}\right)
$$

Usando as regras de Feynman apropriadas, podemos escrever a amplitude correspondente ao diagrama da figura 2.2 no espaço dos momentos. Em seguida tomando o 
quadrado desta amplitude e calculando a seção de choque chegamos a:

$$
\begin{aligned}
\left.\frac{d \sigma_{T, q \bar{q}}^{D}}{d t}\right|_{t=0}= & \frac{\alpha_{e m}}{6 \pi} \sum_{a} e_{a}^{2} \int_{0}^{1} d z\left[z^{2}+(1-z)^{2}\right] \\
& \times \int d^{2} \vec{k} d^{2} \vec{K} d^{2} \vec{K}^{\prime} \alpha_{s}^{2} \frac{f\left(x_{\mathbb{P}}, \vec{K}^{2}\right)}{\vec{K}^{4}} \frac{f\left(x_{\mathbb{P}^{\prime}, \vec{K}^{\prime}}\right)}{\vec{K}^{\prime}} \\
& \times\left\{\frac{\vec{k} \cdot(\vec{k}+\vec{K})}{\left(\vec{k}^{2}+\varepsilon^{2}\right)^{2}}-\frac{\vec{k} \cdot\left(\vec{k}-\vec{K}^{\prime}\right)}{\left(\overrightarrow{k^{2}}+\varepsilon^{2}\right)\left[(\vec{k}+\vec{K})^{2}+\varepsilon^{2}\right]}-\frac{\vec{k}}{\left(\vec{k}{ }^{2}+\varepsilon^{2}\right)\left[\left(\vec{k}-\vec{K}^{\prime}\right)^{2}+\varepsilon^{2}\right]}\right. \\
& \left.+\frac{(\vec{k}+\vec{K}) \cdot\left(\vec{k}-\vec{K}^{\prime}\right)}{\left[(\vec{k}+\vec{K})^{2}+\varepsilon^{2}\right]\left[\left(\vec{k}-\vec{K}^{\prime}\right)^{2}+\varepsilon^{2}\right]}\right\}
\end{aligned}
$$

onde

$$
\varepsilon^{2}=z(1-z) Q^{2}=\frac{\vec{k}^{2} Q^{2}}{M_{X}^{2}}
$$

Na expressão acima podemos distinguir na primeira linha uma integral sobre a fração de momento do fóton carregada pelo quark $(z)$. Esta integral vem da integração sobre o espaço de fase e o termo $z^{2}+(1-z)^{2}$ faz parte do quadrado da função de onda do dipolo. Nas duas últimas linhas aparecem termos provenientes dos propagadores que aparecem na figura. Na segunda linha aparece (ao quadrado !) a integral em $K$ da função $f\left(x_{\mathbb{P}}, \vec{K}^{2}\right) / \vec{K}^{4}$. Como já foi visto em $(2,1.12)$ ela está diretamente relacionada com $\sigma_{d i p}$, a seção de choque de dipolo. Esta função aparece aqui porque foi usado o resultado, previamente obtido no caso do espalhamento inclusivo, que relaciona a interação entre um gluon e o alvo com $\sigma_{d i p}$. Como temos dois gluons ela aparece ao quadrado.

Podemos escrever a expressão $(2,2.54)$ na representação do parâmetro de impacto. Observando que

$$
d z d \vec{k}^{2}=\frac{\vec{k}^{2}}{M_{X}^{4}}\left(1-4 \frac{\vec{k}^{2}}{M_{X}^{2}}\right)^{-1 / 2} d M_{X}^{2} d \vec{k}^{2}
$$


podemos reescrever a equação $(2,2.54)$ como:

$$
\left.\frac{d \sigma_{T, q \bar{q}}^{D}}{d M_{X}^{2} d \vec{k}^{2} d t}\right|_{t=0}=\frac{\pi^{2} \alpha_{e m}}{24} \sum_{q} e_{q}^{2} \frac{1}{M_{X}^{4}}\left(1-4 \frac{\vec{k}^{2}}{M_{X}^{2}}\right)^{-1 / 2}\left(1-2 \frac{\vec{k}^{2}}{M_{X}^{2}}\right) I_{T}^{2}
$$

onde

$$
I_{T}=\int \frac{d \vec{K}^{2}}{\vec{K}^{4}} \alpha_{s} f\left(x_{\mathbb{P}}, \vec{K}^{2}\right)\left[\frac{\vec{k}^{2}-\varepsilon^{2}}{\vec{k}^{2}+\varepsilon^{2}}+\frac{\vec{K}^{2}-\vec{k}^{2}+\varepsilon^{2}}{\sqrt{\left(\vec{K}^{2}+\vec{k}^{2}+\varepsilon^{2}\right)^{2}-4 \vec{K}^{2} \vec{k}^{2}}}\right]
$$

A partir das equações acima podemos derivar $F_{T, L}^{D(3)}$ usando a relação:

$$
x_{\mathbb{P}} F_{T, L}^{D(3)}=\frac{1}{4 \pi \alpha_{e m}} \frac{Q^{4}}{\beta} \frac{d \sigma_{T, L}^{D}}{d M_{X}^{2}} .
$$

Podemos fazer a integração em $t$ assumindo que a dependência nesta variável é exponencial com um slope $B_{D}$. Assim:

$$
x_{\mathbb{P}} F_{T, q \bar{q}}^{D(3)}=\frac{1}{96 B_{D}} \sum_{q} e_{q}^{2} \frac{\beta}{1-\beta} \int d \tilde{k}^{2}\left(1-4 \beta \frac{\tilde{\vec{k}}^{2}}{Q^{2}}\right)^{-1 / 2}\left(1-2 \beta \frac{\tilde{\vec{k}}^{2}}{Q^{2}}\right) I_{T}^{2}
$$

com

$$
\tilde{\vec{k}}^{2}=\frac{\vec{k}^{2}}{1-\beta}=\vec{k}^{2}+\varepsilon^{2}
$$

Quando $z \rightarrow 0, \tilde{\vec{k}}^{2} \simeq-k^{\prime 2}$ (este é o limite que dá a principal contribuição para o DDIS). Esta é a virtualidade do (anti) quark do dipolo, e representa a escala na qual $\alpha_{s}$ será tomada.

Agora, partindo das expressões escritas em função da distribuição de gluons não integradas, vamos reescrever $x_{\mathbb{P}} F_{T, q \bar{q}}^{D(3)}$ em termos da seção de choque de dipolo. Fazendo uma mudança de notação na expressão acima, a função de estrutura difrativa 
fica:

$$
x_{\mathbb{P}} F_{T, q \bar{q}}^{D(3)}=\frac{Q^{2}}{192 B_{D}} \cdot \sum_{q} e_{q}^{2} \cdot \sum_{h, \bar{h}} 2 \int_{z_{0}}^{1 / 2} d z \frac{k_{t}^{2}}{1-\beta}\left\{\left|\int \frac{d^{2} \vec{l}_{t}}{\pi l_{t}^{2}} \alpha_{s} f\left(x_{\mathbb{P}}, l_{t}^{2}\right) \Delta^{0}\right|\right\}^{2}
$$

onde

$$
\Delta^{n}=\left[2 \psi_{h, \bar{h}}^{n}\left(z, \vec{k}_{t}\right)-\psi_{h, \bar{h}}^{n}\left(z, \vec{k}_{t}+\vec{l}_{t}\right)-\psi_{h, \bar{h}}^{n}\left(z, \vec{k}_{t}-\vec{l}_{t}\right)\right]
$$

Seguindo a referência [35], escrevemos:

$$
\begin{aligned}
\int \frac{d^{2} \vec{l}_{t}}{\pi l_{t}^{2}} \alpha_{s} f\left(x_{\mathbb{P}}, l_{t}^{2}\right) \Delta^{n} & \\
& =\int d^{2} \vec{r} \alpha_{s} f\left(x_{\mathbb{P}}, l_{t}^{2}\right) \psi(z, \vec{r})\left[2 e^{i \vec{k}_{t} \cdot \vec{r}}-e^{i\left(\vec{k}_{t}+\vec{l}_{t}\right) \cdot \vec{r}}-e^{i\left(\vec{k}_{t}-\vec{l}_{t}\right) \cdot \vec{r}}\right] \\
& =\int d^{2} \vec{r} \alpha_{s} f\left(x_{\mathbb{P}}, l_{t}^{2}\right) \psi(z, \vec{r}) e^{i \vec{k}_{t}}\left[1-e^{i \vec{l}_{t} \cdot \vec{r}}+1-e^{-i \vec{l}_{t} \cdot \vec{r}}\right] \\
& =\frac{3}{2 \pi^{2}} \int d^{2} \vec{r} \sigma_{d}\left(x_{\mathbb{P}}, r\right) e^{i \vec{k}_{t} \cdot \vec{r}} \psi(z, \vec{r})
\end{aligned}
$$

onde usamos a transformada de Fourier de $\psi\left(z, \vec{k}_{t}\right)$ e a definição da seção de choque de dipolo em termos de $f$. Fazendo as integrações angulares na expressão acima obtemos finalmente a contribuição transversal da componente $q \bar{q}$ para a função de estrutura:

$$
\begin{aligned}
x_{\mathbb{P}} F_{T}^{D}= & \frac{3 Q^{2}}{128 \pi^{4} B_{D}} \sum_{f} e_{f}^{2} 2 \int_{z_{0}}^{1 / 2} d z z(1-z) \\
& \times\left\{\varepsilon_{f}^{2}\left[z^{2}+(1-z)^{2}\right]\left[\int d r r K_{1}\left(\varepsilon_{f} r\right) \sigma_{d}\left(x_{\mathbb{P}}, r\right) J_{1}\left(k_{t} r\right)\right]^{2}\right. \\
& \left.+m_{f}^{2}\left[\int d r r K_{0}\left(\varepsilon_{f} r\right) \sigma_{d}\left(x_{\mathbb{P}}, r\right) J_{0}\left(k_{t} r\right)\right]^{2}\right\}
\end{aligned}
$$




\section{A componente quark-antiquark longitudinal}

Repetindo os passos anteriores partimos da seção de choque diferencial dada por

$$
\begin{aligned}
\left.\frac{d \sigma_{L, q \bar{q}}^{D}}{d t}\right|_{t=0}= & \frac{\alpha_{e m}}{6 \pi} \sum_{a} e_{a}^{2} \int_{0}^{1} d z 4 Q^{2} z^{2}(1-z)^{2} \\
& \times \int d^{2} \vec{k} d^{2} \vec{K} d^{2} \vec{K}^{\prime} \alpha_{s}^{2} \frac{f\left(x_{\mathbb{P}^{\prime}}, \vec{K}^{2}\right)}{\vec{K}^{4}} \frac{f\left(x_{\mathbb{P}}, \vec{K}^{\prime 2}\right)}{\vec{K}^{\prime}} \\
& \times\left\{\frac{1}{\left(\vec{k}^{2}+\varepsilon^{2}\right)}-\frac{1}{\left(\vec{k}^{2}+\varepsilon^{2}\right)\left[(\vec{k}+\vec{K})^{2}+\varepsilon^{2}\right]}-\frac{1}{\left(\vec{k}^{2}+\varepsilon^{2}\right)\left[\left(\vec{k}-\vec{K}^{\prime}\right)^{2}+\varepsilon^{2}\right]}\right. \\
& \left.+\frac{1}{\left[(\vec{k}+\vec{K})^{2}+\varepsilon^{2}\right]\left[\left(\vec{k}-\vec{K}^{\prime}\right)^{2}+\varepsilon^{2}\right]}\right\} .
\end{aligned}
$$

Através de uma mudança de variáveis chegamos a:

$$
\left.\frac{d \sigma_{L, q \bar{q}}^{D}}{d M_{X}^{2} d \vec{k}^{2} d t}\right|_{t=0}=\frac{2 \pi^{2} \alpha_{e m}}{3} \sum_{q} e_{q}^{2} \frac{Q^{2}}{M_{X}^{4}\left(M_{X}^{2}+Q^{2}\right)^{2}} \vec{k}^{2}\left(1-4 \frac{\vec{k}^{2}}{M_{X}^{2}}\right)^{-1 / 2} I_{L}^{2}
$$

onde

$$
I_{L}=\int \frac{d \vec{K}^{2}}{\vec{K}^{4}} \alpha_{s} f\left(x_{\mathbb{P}}, \vec{K}^{2}\right)\left[1-\frac{\vec{k}^{2}+\varepsilon^{2}}{\sqrt{\left(\vec{K}^{2}+\vec{k}^{2}+\varepsilon^{2}\right)^{2}-4 \vec{K}^{2} \vec{k}^{2}}}\right]
$$

Usando $(2,2.58)$ e fazendo a integral na variável $t$ chegamos a:

$$
x_{I P} F_{L, q \bar{q}}^{D(3)}=\frac{1}{6 b} \sum_{q} e_{q}^{2} \beta^{3} \int d \tilde{\vec{k}}^{2} \frac{\tilde{\vec{k}}^{2}}{Q^{2}}\left(1-4 \beta \frac{\tilde{\vec{k}}^{2}}{Q^{2}}\right)^{-1 / 2} I_{L}^{2}
$$

onde $\tilde{\vec{k}}^{2}$ está definido em $(2,2.60)$.

Agora vamos escrever $x_{\mathbb{P}} F_{L, q \bar{q}}^{D(3)}$ em termos da seção de choque de dipolo. Usando expressões semelhantes as equações $(2,2.61),(2,2.62)$ e $(2,2.63)$, e fazendo as integra- 


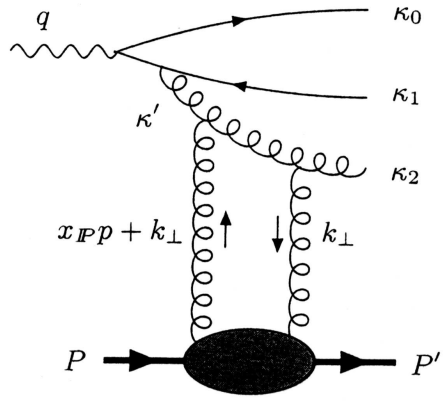

(a)

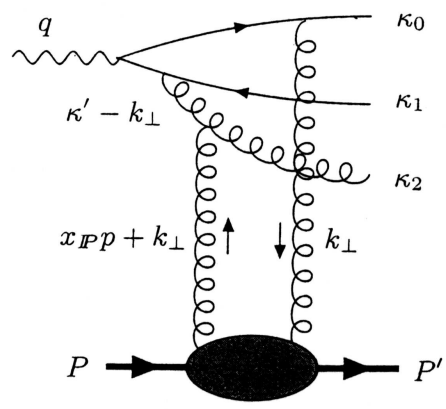

(b)

Figura 2.3: Produção difrativa $q \bar{q} g$.

ções angulares (que dão origem às funções de Bessel), chegamos finalmente a:

$$
\begin{aligned}
x_{\mathbb{P}} F_{L, q \bar{q}}^{D(3)}= & \frac{3 Q^{4}}{64 \pi^{4} B_{D}} \sum_{f} e_{f}^{2} 4 \int_{z_{0}}^{1 / 2} d z z^{2}(1-z)^{2} \\
& \times \frac{k_{t}^{2}}{1-\beta}\left[\int d r r K_{0}\left(\varepsilon_{f}, r\right) \sigma_{d}\left(x_{\mathbb{P}}, r\right) J_{0}\left(k_{t} r\right)\right]^{2} \\
= & \frac{3 Q^{6}}{32 \pi^{4} B_{D}} \sum_{f} e_{f}^{2} 2 \int_{z_{0}}^{1 / 2} d z z^{3}(1-z)^{3} \\
& \times\left[\int d r r K_{0}\left(\varepsilon_{f}, r\right) \sigma_{d}\left(x_{\mathbb{P}}, r\right) J_{0}\left(k_{t} r\right)\right]^{2}
\end{aligned}
$$

onde foram usadas as relações:

$$
k_{t}^{2}+z(1-z) Q^{2}=k^{2} ; \frac{k_{t}^{2}}{1-\beta}=k^{2} ; k^{2}=\frac{Q^{2}}{\beta} z(1-z) .
$$

\section{A componente quark-antiquark-gluon}

A parametrização de Sudakov para o momento do quark, do antiquark e do gluon é (Veja a figura 2.3.) [29, 37]: 


$$
\begin{aligned}
& k_{0}=\left(1-z_{1}-z_{2}\right) q^{\prime}+\frac{\left(\overrightarrow{k_{1}}+\overrightarrow{k_{2}}\right)^{2}}{\left(1-z_{1}-z_{2}\right) W^{2}} P\left(k_{1 \perp}+k_{2 \perp}\right) \\
& k_{1}=z_{1} q^{\prime}+\frac{\vec{k}_{1}^{2}}{z_{1} W^{2}} P k_{1 \perp} \\
& k_{2}=z_{2} q^{\prime}+\frac{\vec{k}_{2}^{2}}{z_{2} W^{2}} P k_{2 \perp}
\end{aligned}
$$

A massa invariante para o estado $q \bar{q} g$ é:

$$
M_{X}^{2}=\frac{{\overrightarrow{k_{1}}}^{2}}{z_{1}}+\frac{{\overrightarrow{k_{2}}}^{2}}{z_{2}}+\frac{\left(\overrightarrow{k_{1}}+\overrightarrow{k_{2}}\right)^{2}}{\left(1-z_{1}-z_{2}\right)}
$$

Em termos da massa invariante do estado $q \bar{q}, m_{q \bar{q}}^{2}=\left(k_{0}+k_{1}\right)^{2}$, temos:

$$
M_{X}^{2}=\frac{z_{2} m_{q \bar{q}}^{2}+{\overrightarrow{k_{2}}}^{2}}{z_{2}\left(1-z_{2}\right)} .
$$

O momento do glúon emitido pelo dipolo $q \bar{q}$ é:

$$
k^{\prime}=k_{2}-x_{\mathbb{P}} P=z_{2} q^{\prime}-\left(1-\frac{\vec{k}^{2}}{z_{2} W^{2} x_{\mathbb{P}}}\right) x_{\mathbb{P}} P+k_{2 \perp} .
$$

Definimos $y$ como sendo a fração de momento do Pomeron $x_{\mathbb{P}} P$ carregada pelo glúon:

$$
y=1-\frac{{\overrightarrow{k_{2}}}^{2}}{z_{2} W^{2} x_{\mathbb{P}}},
$$

e a virtualidade do glúon é:

$$
k^{\prime 2}=-\frac{\vec{k}_{2}^{2}}{1-y}
$$

A seção de choque difrativa proveniente da contribuição da componente de Fock $q \bar{q} g$ do fóton foi investigada em vários regimes cinemáticos. Na aproximação de leading twist e $\ln Q^{2}$, a separação transversal entre o quark e o anti-quark é muito menor do que a distância entre os quarks e o gluon. Assim, o par $q \bar{q}$ e o glúon formam um dipolo 


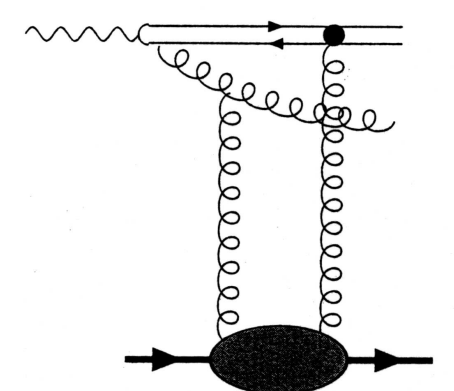

Figura 2.4: Contribuição do dipolo de cor efetivo.

de cor efetivo, como mostrado na figura 2.4.

A componente $q \bar{q} g$ da função de estrutura difrativa é:

$$
\begin{aligned}
x_{\mathbb{P}} F_{q \bar{q} g}^{D(3)}= & \frac{9}{64 b} \sum_{q} e_{q}^{2} \beta \int d \tilde{\vec{k}}^{2} \frac{\alpha_{s}}{2 \pi} \ln \frac{Q^{2}}{\tilde{\vec{k}}^{2}} \\
& \times \int_{\beta}^{1} \frac{d y}{y^{2}(1-y)^{2}}\left[\left(1-\frac{\beta}{y}\right)^{2}+\left(\frac{\beta}{y}\right)^{2}\right] I_{g}^{2}
\end{aligned}
$$

onde

$$
\begin{aligned}
I_{g}= & \int \frac{d \vec{K}^{2}}{\vec{K}^{4}} \alpha_{s} f\left(x_{\mathbb{P}}, \vec{K}^{2}\right)\left[y^{2}+(1-y)^{2}+\frac{\vec{K}^{2}}{\tilde{\vec{k}}^{2}}\right. \\
& \left.-\frac{\left[(1-2 y) \tilde{\vec{k}}^{2}-\vec{K}^{2}\right]^{2}+2 y(1-y) \stackrel{\tilde{\vec{k}}}{\hat{k}}}{\tilde{\overrightarrow{k^{2}}} \sqrt{\left(\tilde{\vec{k}}^{2}+\vec{K}^{2}\right)^{2}-4(1-y) \vec{K}^{2} \tilde{\vec{k}}^{2}}}\right] .
\end{aligned}
$$

A variável $\tilde{\vec{k}}^{2}$ usada aqui é agora: $\tilde{\vec{k}}^{2}=\frac{\vec{k}^{2}}{1-y}$ e está relacionada com a virtualidade do glúon no dipolo efetivo.

Seguimos agora os passos das seções anteriores e escrevemos a componente $q \bar{q} g$ da seção de choque e depois passamos para a componente correspondente da função de estrutura em termos da distribuição de gluons não integrada, a qual, depois de uma 
redefinição de variáveis torna-se:

$$
\begin{aligned}
x_{\mathbb{P}} F_{T, q \bar{q} g}^{D(3)}\left(Q^{2}, \beta, x_{\mathbb{P}}\right)= & \frac{9 \beta}{64 B_{D}} \sum_{f} e_{f}^{2} \int_{0}^{Q^{2}} d k^{2} \frac{\alpha_{s}}{2 \pi} \ln \left(\frac{Q^{2}}{k^{2}}\right) \int_{\beta}^{1} \frac{d \theta}{\theta^{2}(1-\theta)^{2}} \\
& \times\left[\left(1-\frac{\beta}{\theta}\right)^{2}+\left(\frac{\beta}{\theta}\right)^{2}\right]\left\{\int \frac{d l_{t}^{2}}{l_{t}^{2}} \alpha_{s} f\left(x_{\mathbb{P}}, l_{t}^{2}\right)\right. \\
& \left.\times\left[\theta^{2}+(1-\theta)^{2}+\frac{l_{t}^{2}}{k^{2}}-\frac{\left[(1-2 \theta) k^{2}-l_{t}^{2}\right]^{2}+2 \theta(1-\theta) k^{4}}{k^{2} \sqrt{\left(k^{2}+l_{t}^{2}\right)^{2}-4(1-\theta) l_{t}^{2} k^{2}}}\right]\right\}^{2} .
\end{aligned}
$$

O termo ao quadrado de $(2,2.79)$ está relacionado à função de onda do fóton da seguinte maneira:

$$
\begin{aligned}
& \int \frac{d l_{t}^{2}}{l_{t}^{2}} \alpha_{s} f\left(x_{\mathbb{P}}, l_{t}^{2}\right)\left[2 \psi^{m, n}\left(z, \vec{k}_{t}\right)-\psi^{m, n}\left(z, \vec{k}_{t}+\vec{l}_{t}\right)-\psi^{m, n}\left(z, \vec{k}_{t}-\vec{l}_{t}\right)\right] \\
= & \int \frac{d l_{t}^{2}}{l_{t}^{2}} \frac{\alpha_{s} f\left(x_{\mathbb{P}}, l_{t}^{2}\right)}{(1-\theta) \sqrt{\theta k^{2}}}\left[\theta^{2}+(1-\theta)^{2}+\frac{l_{t}^{2}}{k^{2}}\right. \\
& \left.-\frac{\left[(1-2 \theta) k^{2}-l_{t}^{2}\right]^{2}+2 \theta(1-\theta) k^{4}}{k^{2} \sqrt{\left(k^{2}+l_{t}^{2}\right)^{2}-4(1-\theta) l_{t}^{2} k^{2}}}\right]\left\{\delta^{m n}-2 \frac{\vec{k}_{t}^{m} \vec{k}_{t}^{n}}{k_{t}^{2}}\right\}
\end{aligned}
$$

$\mathrm{Na}$ integral envolvendo a função de onda do fóton, a distribuição de gluons não integrada pode ser substituída pela seção de choque de dipolo:

$$
\begin{aligned}
& \int \frac{d l_{t}^{2}}{l_{t}^{2}} \alpha_{s} f\left(x_{\mathbb{P}}, l_{t}^{2}\right)\left[2 \psi^{m, n}\left(z, \vec{k}_{t}\right)-\psi^{m, n}\left(z, \vec{k}_{t}+\vec{l}_{t}\right)-\psi^{m, n}\left(z, \vec{k}_{t}-\vec{l}_{t}\right)\right] \\
= & 2 \int d^{2} \vec{r} \psi^{m n}(z, \vec{r}) e^{i \vec{k}_{t} \cdot \vec{r}} \frac{3}{4 \pi^{2}} \sigma_{d i p}\left(r, x_{\mathbb{P}}\right)
\end{aligned}
$$


Assim o termo quadrático da equação $(2,2.79)$ pode ser substituído por

$$
\begin{aligned}
x_{\mathbb{P}} F_{T, q \bar{q} g}^{D(3)}\left(Q^{2}, \beta, x_{\mathbb{P}}\right)= & \frac{9 \beta}{64 B_{D}} \sum_{f} e_{f}^{2} \int_{0}^{Q^{2}} d k^{2} \frac{\alpha_{s}}{2 \pi} \ln \left(\frac{Q^{2}}{k^{2}}\right) \int_{\beta}^{1} \frac{d \theta}{\theta^{2}(1-\theta)^{2}} \\
& \times\left[\left(1-\frac{\beta}{\theta}\right)^{2}+\left(\frac{\beta}{\theta}\right)^{2}\right] \\
& \times 2\left[\int d^{2} \vec{r} \psi^{m n}(z, \vec{r}) e^{i \vec{k}_{t} \cdot \vec{r}} \frac{3}{4 \pi^{2}} \sigma_{d i p}\left(r, x_{\mathbb{P}}\right)\right]^{2}(1-\theta)^{2} k^{2} .
\end{aligned}
$$

Substituindo

$$
k^{2}=\frac{k_{t}^{2}}{1-\theta} \text { e } d k^{2}=\frac{d k_{t}^{2}}{1-\theta}
$$

em $\theta$ fixo, e também substituindo a expressão da função de onda do fóton por

$$
\psi^{m n}(z, \vec{r})=-\frac{1}{2 \pi}\left(\delta^{m n}-2 \frac{\vec{r}_{t}^{m} \vec{r}_{t}^{n}}{r^{2}}\right) \sqrt{z Q^{2}} K_{2}\left(\sqrt{z Q^{2} r^{2}}\right)
$$

podemos fazer a integração angular e chegar à forma final da componente $q \bar{q} g$ transversal da função de estrutura difrativa:

$$
\begin{aligned}
x_{\mathbb{P}} F_{t, q \bar{q} g}^{D(3)}\left(Q^{2}, \beta, x_{\mathbb{P}}\right)= & \frac{81 \beta \alpha_{s}}{512 \pi^{5} B_{D}} \sum_{f} e_{f}^{2} \int_{\beta}^{1} \frac{d \theta}{(1-\theta)^{3}}\left[\left(1-\frac{\beta}{\theta}\right)^{2}+\left(\frac{\beta}{\theta}\right)^{2}\right] \\
& \times \int_{0}^{(1-\theta) Q^{2}} d k_{t}^{2} \ln \left(\frac{(1-\theta) Q^{2}}{k_{t}^{2}}\right) \\
& \times\left[\int_{0}^{\infty} u d u \sigma_{d i p}\left(u / k_{t}, \tilde{s}\right) K_{2}\left(\sqrt{\frac{\theta}{1-\theta} u^{2}}\right) J_{2}(u)\right]^{2} .
\end{aligned}
$$

onde $u=k_{t} r$.

Onde a equação final para a função de estrutura difrativa $F_{2}^{D(3)}$ é

$$
F_{2}^{D(3)}=F_{L, q \bar{q}}^{D(3)}+F_{T, q \bar{q}}^{D(3)}+F_{T, q \bar{q} g}^{D(3)}
$$




\section{Capítulo 3}

\section{Equações não lineares de evolução}

\subsection{Introdução}

O Condensado de Vidros Coloridos (Color Glass Condensate - CGC) é formado por um sistema de alta densidade hadrônica, que controla as interações em QCD à altas energias, ou pequenos valores da variável $x$ de Bjorken. Previsto a partir de considerações teóricas, este sistema pode ter sido visto em recentes experimentos no HERA e no RHIC. Acreditamos que no LHC (Large Hadron Collider), será mais fácil observá-lo. O CGC é colorido uma vez que ele é composto de gluons, os quais carregam carga de "cor"; ele é um vidro uma vez que sua dinâmica interna está congelada. Sua escala de tempo natural é muito maior do que a escala de tempo do espalhamento em altas energias. Ele é um condensado pois é caracterizado por um grande número de ocupação e fortes campos coloridos clássicos. A alta densidade e a liberdade assintótica implicam que o CGC é fracamente acoplado. Com base nessa observação, uma teoria efetiva foi construída para descrever a evolução da função de onda hadrônica com o aumento da energia na presença de efeitos não-lineares associados com a alta densidade de gluons [21, 22]. Essa teoria prediz que a distribuição de gluons satura para um momento transversal abaixo de uma escala chamada "escala de saturação", que cresce com a energia. A saturação é atingida quando o aumento do número de pártons cessa 
e um aumento em energia não mais corresponde a um aumento no número de pártons. Como foi visto no Capítulo 1, isso fornece uma explicação natural para algumas características observadas nos dados experimentais, o "scaling geométrico" nos dados de HERA de DIS na região de $x$ pequeno, ou a "supressão de alto- $p_{T}$ " nas colisões deuteron-ouro em RHIC.

Nas equações DGLAP [38] e BFKL [39] supomos que o sistema permanece diluído durante da evolução e as correlações entre os partons podem ser desprezadas. O rápido crescimento da densidade de gluons previsto pelas equações DGLAP e BFKL e experimentalmente observado no HERA leva a uma situação na qual partons individuais eventualmente se sobrepoem, e portanto efeitos de recombinação precisam ser incluídos na evolução. Esses efeitos entram na evolução não-linearmente e inibem o forte crescimento da densidade de gluons.

Como foi mencionado no capítulo 1 , os efeitos de saturação foram primeiro discutidos por Gribov, Levin e Ryskin [20] em 1983 e posteriormente por Mueller e Qiu [40] em 1986. Gribov, Levin e Ryskin apresentaram a idéia de que a energias muito altas o número de gluons seria tão grande que o processo de recombinação de gluons, $g+g \rightarrow g$, passaria a ser importante. Eles propuseram a primeira equação de evolução não linear:

$$
Q^{2} \frac{\partial^{2} x g\left(x, Q^{2}\right)}{\partial \ln (1 / x) \partial Q^{2}}=\frac{\alpha_{S} N_{c}}{\pi} x g\left(x, Q^{2}\right)-\frac{4 \alpha_{S}^{2} N_{c}}{3 C_{F} R^{2}} \frac{1}{Q^{2}}\left[x g\left(x, Q^{2}\right)\right]^{2}
$$

que é válida nos limites $\ln (1 / x) \gg 1$ e $\ln Q^{2} \gg 1$. Observando o sinal negativo do termo não-linear, vemos que o crescimento acentuado de $x g\left(x, Q^{2}\right)$ é atenuado pelo termo responsável pela recombinação de gluons.

A partir da equação GLR podemos estimar a escala na qual os efeitos não lineares contribuem. A saturação ocorrerá quando os termos não-linear e linear se tornarem idênticos. Isso ocorrerá para $Q^{2} \equiv Q_{s}^{2}$ :

$$
Q_{s}^{2}=\frac{4 \pi \alpha_{s}}{3 C_{F} R^{2}} x g\left(x, Q^{2}\right)
$$


Esta escala é denominada escala de saturação. O regime caracterizado por $Q^{2}>Q_{s}^{2}$ é o regime linear, enquanto para $Q^{2}<Q_{s}^{2}$ temos o regime não-linear ou de saturação.

Mais tarde, em 1994, McLerran e Vegunopalan [4, 5] argumentaram que o regime de alta densidade de um objeto hadrônico (um hádron ou um núcleo) pode ser descrito em termos de um conjunto de campos gluônicos clássicos e que o número de glúons com momento menor do que a escala de saturação é tão alto quanto ele pode ser (ou seja saturado). O estudo da evolução de um objeto hadrônico realizado por JalilianMarian, Kovner, Leonidov, Weigert, McLerran e Iancu deu origem à famosa equação JIMWLK [21, 22], a qual é escrita em termos de uma equação funcional não linear.

Uma derivação alternativa, seguida por Balitsky, faz uso da expansão do produto de operadores (OPE) para QCD em altas energias para derivar uma hierarquia de equações de evolução acopladas. No limite de grande número de cor, essa hierarquia reduz-se a uma equação. Esta equação foi derivada independentemente por Kovchegov no modelo de dipolo para espalhamento em altas energias.

A relação entre essas duas aproximações tem sido extensivamente estudada. Elas levam ao mesmo resultado, conhecido como a equação de Balitsky-Kovchegov (BK). Esta equação tem servido como ponto de partida para inúmeros estudos analíticos e numéricos [41].

Embora não se conheça ainda uma solução analítica completa da equação BK, muitas das suas propriedades, tais como a existência e a forma das soluções assintóticas, foram identificadas em estudos analíticos e numéricos. Muitos deles referem-se ao caso da constante de acoplamento fixa e sem dependência no parâmetro de impacto. No entanto já existem trabalhos numéricos que consideram a constante de acoplamento "running" e a dependência no parâmetro de impacto [41, 42].

\subsection{A equação de Balitsky-Kovchegov}

A equação BK [43] descreve a evolução em rapidez $Y=\ln \left(s / s_{0}\right)=\ln \left(x_{0} / x\right)$ da probabilidade de espalhamento $N(\vec{x}, \vec{y}, Y)$ de um dipolo $q \bar{q}$ com o alvo hadrônico, 


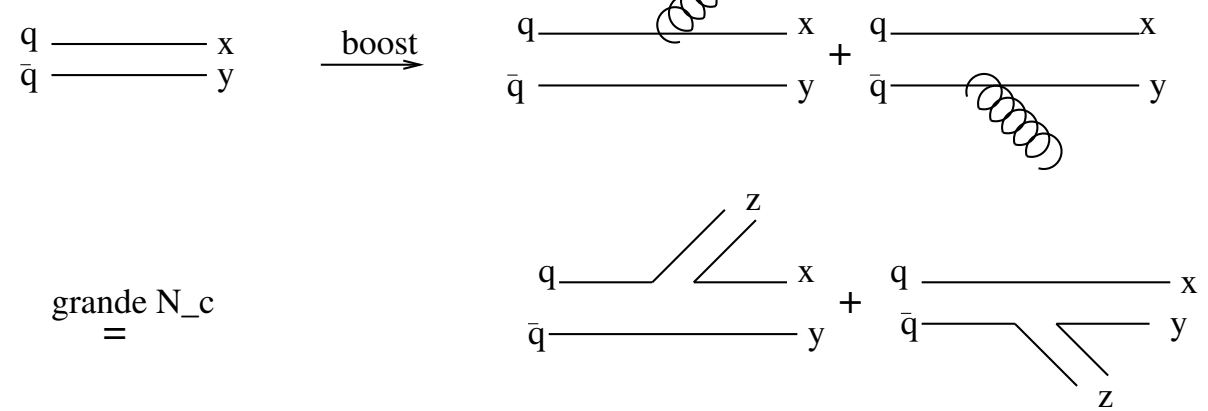

Figura 3.1: Diagrama para a emissão de um glúon na evolução de um dipolo e seu limite de $N_{c} \rightarrow \infty$.

onde $\vec{x}(\vec{y})$ é a posição do quark $q$ (anti-quark $\bar{q}$ ) no espaço transversal com respeito ao centro do alvo. Definimos (Veja figura 3.1.)

$$
\begin{aligned}
\vec{r} & =\vec{x}-\vec{y}, \\
\overrightarrow{r_{1}} & =\vec{x}-\vec{z}, \\
\overrightarrow{r_{2}} & =\vec{y}-\vec{z} \\
b & =\frac{\vec{x}+\vec{y}}{2} .
\end{aligned}
$$

Se desprezarmos a dependência no parâmetro de impacto (justificada quando o alvo homogêneo tem raio muito maior do que qualquer tamanho de dipolo considerado), a equação BK fica $(r \equiv|\vec{r}|)$

$$
\begin{aligned}
\frac{\partial N(r, Y)}{\partial Y}= & \int \frac{d^{2} z}{2 \pi} K\left(\vec{r}, \overrightarrow{r_{1}}, \overrightarrow{r_{2}}\right)\left[N\left(r_{1}, Y\right)+N\left(r_{2}, Y\right)\right. \\
& \left.-N(r, Y)-N\left(r_{1}, Y\right) N\left(r_{2}, Y\right)\right],
\end{aligned}
$$


onde o "kernel" BFKL é

$$
\begin{aligned}
K\left(\vec{r}, \overrightarrow{r_{1}}, \overrightarrow{r_{2}}\right) & =\bar{\alpha}_{s} \frac{r^{2}}{r_{1}^{2} r_{2}^{2}} \\
\bar{\alpha}_{s} & =\frac{\alpha_{s} N_{c}}{\pi} .
\end{aligned}
$$

A constante de acoplamento é fixa.

A equação BK tem a seguinte interpretação probabilística (figura 3.1): quando evoluído em rapidez (ou, em outras palavras, quando observado com uma energia maior) o dipolo pai com finais localizados em $\vec{x}$ e $\vec{y}$ emite um glúon, o qual corresponde no limite de grande $N_{c}$ a dois dipolos com finais $(\vec{x}, \vec{z})$ e $(\vec{z}, \vec{y})$, respectivamente. A probablidade da emissão é dada pelo kernel BFKL $(3,2.4)$ e multiplicada pela probabilidade de espalhamento dos novos dipolos menos a probabilidade de espalhamento do dipolo pai. O termo não linear é subtraído para evitar a dupla contagem. É este termo não linear que contrasta com a equação BFKL, sendo responsável pelo crescimento limitado da amplitude em rapidez. A equação BK assegura unitariedade localmente na configuração de espaço transversal, $|N(r, Y)| \leq 1$. Isto é garantido pois para $N(r, Y)=1$, a derivada com respeito a $Y$ em $(3,2.3)$ não pode ser positiva.

No limite de $Y \rightarrow \infty$, as soluções da equação BK exibem o fenômento de scaling geométrico [44], ou seja, as soluções não são mais funções das variáveis de $r$ e $Y$ separadamente, e dependem de uma única variável:

$$
\tau \equiv r Q_{s}(Y)
$$

Nesta região $Q_{s}(Y) \gg \Lambda_{Q C D}$, a equação $\mathrm{BK}(3,2.3)$ para constante de acoplamento fixa pode ser escrita em termos das variáveis $\vec{\tau}=Q_{s} \vec{r}, \vec{\tau}_{1}=Q_{s} \vec{r}_{1}$ e $\vec{\tau}_{2}=Q_{s} \vec{r}_{2}$. A dependência em $Y$ de $N(r, Y)$ está contida em $Q_{s}(Y), N(r, Y) \equiv N(\tau)$. Reescrevendo a derivada do lado esquerdo de $(3,2.3)$, temos

$$
\frac{\partial N(\tau)}{\partial Y}=\frac{\partial N}{\partial \tau} \frac{\partial \tau}{\partial Y}=\frac{\partial Q_{s}(Y)}{\partial Y} r \frac{\partial N}{\partial \tau}=\frac{\partial \ln \left[Q_{s}^{2}(Y) / \Lambda_{Q C D}^{2}\right]}{\partial Y} r^{2} \frac{\partial N}{\partial r^{2}}
$$


Integrando a equação acima em $d^{2} r / r^{2}$, temos

$$
\int \frac{d^{2} r}{r^{2}} \frac{\partial N(\tau)}{\partial Y}=\pi \frac{\partial \ln \left[Q_{s}(Y) / \Lambda_{Q C D}^{2}\right]}{\partial Y}[N(\infty)-N(0)]=\pi \frac{\partial \ln \left[Q_{s}(Y) / \Lambda_{Q C D}^{2}\right]}{\partial Y}
$$

onde usamos as condições de contorno $N(\infty)=1$ e $N(0)=0$. Fazendo a mesma integração do lado direito de $(3,2.3)$ e lembrando que

$$
\frac{d^{2} r}{r^{2}}=\frac{d^{2} \tau}{\tau^{2}}
$$

temos que o lado direito de $(3,2.3)$ será:

$$
\int \frac{d^{2} \tau d^{2} \tau_{1}}{2 \pi} \bar{\alpha}_{s} \frac{1}{\tau_{1}^{2} \tau_{2}^{2}}\left[N\left(\tau_{1}\right)+N\left(\tau_{2}\right)-N(\tau)-N\left(\tau_{1}\right) N\left(\tau_{2}\right)\right]
$$

Igualando $(3,2.7)$ com $(3,2.9)$, temos:

$$
\frac{\partial \ln \left[Q_{s}^{2}(Y) / \Lambda_{Q C D}^{2}\right]}{\partial Y}=\bar{\alpha}_{s} \int \frac{d^{2} \tau d^{2} \tau_{1}}{2 \pi^{2}} \frac{1}{\tau_{1}^{2} \tau_{2}^{2}}\left[N\left(\tau_{1}\right)+N\left(\tau_{2}\right)-N(\tau)-N\left(\tau_{1}\right) N\left(\tau_{2}\right)\right]
$$

O integrando é independente de $Y$, então

$$
\frac{\partial \ln \left[Q_{s}^{2}(Y) / \Lambda_{Q C D}^{2}\right]}{\partial Y}=d \bar{\alpha}_{s}
$$

Resolvendo para $Q_{s}$

$$
Q_{s}^{2}=\Lambda_{Q C D}^{2} \exp \left(d \bar{\alpha}_{s} Y\right)
$$

a qual demonstra que a escala de saturação cresce com a energia. Portanto esperamos que no limite de altas energias as seções de choque sejam determinadas pela física de saturação. Essa é a forma da escala de saturação dependente da rapidez para o caso da constante de acoplamento fixa.

Agora voltamos para a equação BK $(3,2.3)$ e a escrevemos em termos de $S$, onde $S$ é o elemento da matriz do espalhamento dipolo-hádron, que está relacionada com a 
amplitude de espalhamento dipolo-hádron $N$ por:

$$
S=1-N
$$

Assim a equação BK escrita em termos de $S$ fica:

$$
\frac{\partial S(\vec{x}-\vec{y})}{\partial Y}=-\int \frac{d^{2} z}{2 \pi} K(\vec{x}-\vec{y}, \vec{x}-\vec{z}, \vec{y}-\vec{z})[S(\vec{x}-\vec{y})-S(\vec{x}-\vec{z}) S(\vec{y}-\vec{z})]
$$

onde

$$
K(\vec{x}-\vec{y}, \vec{x}-\vec{z}, \vec{y}-\vec{z})=\frac{(\vec{x}-\vec{y})^{2}}{(\vec{x}-\vec{z})^{2}(\vec{y}-\vec{z})^{2}}
$$

No regime de grandes dipolos (regime de saturação), $r_{\perp} \gg 1 / Q_{s}(Y)$, ou seja, $S\left(r_{\perp}\right) \ll$ 1 a equação acima pode ser linearizada, desprezando o termo quadrático em $S$; isso é válido porque a contribuição dominante vem de $z_{\perp}$ satisfazendo: $1 / Q_{s}(Y) \ll \mid z_{\perp}-$ $x_{\perp} \mid \ll r_{\perp}$. Assim a equação $(3,2.14)$ fica:

$$
\frac{\partial S}{\partial Y}=-\bar{\alpha}_{s} S\left(r_{\perp}\right) \int_{1 / Q_{s}}^{r_{\perp}} \frac{d^{2} z}{2 \pi} \frac{r_{\perp}^{2}}{z_{\perp}^{2}\left(r_{\perp}-z_{\perp}\right)^{2}}
$$

Integrando em $z_{\perp}$ resulta:

$$
\frac{\partial S}{\partial Y} \sim \bar{\alpha}_{s} S\left(r_{\perp}\right) \ln \left(r_{\perp}^{2} Q_{s}^{2}\right)
$$

Definindo a variável de scaling: $\xi \equiv \ln \left[r_{\perp}^{2} Q_{s}^{2}(Y)\right]$ e fazendo

$$
\frac{\partial \xi}{\partial Y}=\frac{\partial \ln \left[r_{\perp}^{2} Q_{s}^{2}(Y)\right]}{\partial Y}=c \bar{\alpha}_{s}
$$

temos

$$
\frac{\partial S}{\partial Y}=\frac{\partial S}{\partial \xi} \frac{\partial \xi}{\partial Y}
$$

Logo,

$$
\frac{\partial S}{\partial \xi} c \bar{\alpha}_{s}=-\bar{\alpha}_{s} \ln \left[r_{\perp}^{2} Q_{s}^{2}(Y)\right] S\left(r_{\perp}\right)
$$


e portanto

$$
\frac{\partial S}{\partial \xi}=-\frac{1}{c} \xi S
$$

Resolvendo a equação acima para $S$ obtemos:

$$
S(\xi)=S_{0} e^{-\xi^{2} / 2 c}
$$

Agora voltando para a amplitude de espalhamento dipolo-hádron $N$, temos que

$$
N\left(r_{\perp}, Y\right)=1-S_{0} e^{-l n^{2}\left[r_{\perp}^{2} Q_{s}^{2}(Y)\right] / 2 c},
$$

que é conhecida como lei de Levin-Tuchin [45], a qual apresenta o escalamento geométrico.

\subsection{A equação de BFKL no limite de saturação}

No regime linear a equação BK (3,2.3), depois de algumas manipulações, se reduz a:

$$
\frac{\partial N\left(r_{\perp}\right)}{\partial Y}=-\bar{\alpha}_{s} \int \frac{d^{2} z}{\pi} \frac{r_{\perp}^{2}}{z_{\perp}^{2}\left(r_{\perp}-z_{\perp}\right)^{2}}\left(N\left(z_{\perp}\right)-\frac{1}{2} N\left(r_{\perp}\right)\right)
$$

que é a equação de BFKL. Aqui iremos reconsiderar a solução desta equação considerando a condição de contorno $N\left(r_{\perp}\right) \sim 1$ para $r_{\perp} \sim 1 / Q_{s}(Y)$, ou seja, a existência de um regime de saturação [46].

Para obtermos a solução desta equação é conveniente usarmos a transformada de Mellin com respeito a coordenada transversal:

$$
N\left(r_{\perp}\right)=\int \frac{d \gamma}{2 \pi i}\left(\frac{r_{\perp}^{2}}{l^{2}}\right)^{\gamma} N(\gamma)
$$

onde $l^{2}=1 / \Lambda^{2}$ e $r_{\perp}^{2} \ll l^{2}$. Uma vez que a equação de BFKL é invariante por 
transformações de escala, a equação que resulta para $N(\gamma)$ é local em $\gamma$ :

$$
\frac{\partial N(\gamma)}{\partial Y}=\bar{\alpha}_{s} \chi(\gamma) N(\gamma)
$$

onde

$$
\chi(\gamma)=2 \Psi(1)-\Psi(\gamma)-\Psi(1-\gamma)
$$

sendo $\Psi$ a função di-gamma. A solução de $(3,3.25)$ é:

$$
N(\gamma)=e^{\bar{\alpha}_{s} \chi(\gamma) Y} N_{0}(\gamma)
$$

Para retornar às coordenadas espaciais, devemos calcular a integral:

$$
N=\int_{C} \frac{d \gamma}{2 \pi i} e^{\gamma r} e^{\bar{\alpha}_{s} \chi(\gamma) Y} N_{0}(\gamma)=\int_{C} \frac{d \gamma}{2 \pi i} e^{F(\gamma, r, Y)}
$$

onde $r=\ln \left(r_{\perp}^{2} / l^{2}\right)$ é negativo, pois $r_{\perp}^{2} \ll l^{2}$ e definimos $F(\gamma, r, Y) \equiv r \gamma+\bar{\alpha}_{s} \chi(\gamma) Y$. Esta integral pode ser calculada na aproximação de ponto de sela. A condição inicial $N_{0}(\gamma)$ será considerada como um valor constante, uma vez que sua contribuição para a função $F(\gamma, r, Y)$ no expoente não fica mais importante do que $r$ ou $\bar{\alpha}_{s} Y$. Assim obtemos $\left(\gamma=\gamma_{0}+i \nu\right)$ :

$$
N \simeq e^{F\left(\gamma_{0}\right)} \int_{-\infty}^{\infty} \frac{d \nu}{2 \pi} e^{-\frac{1}{2} \nu^{2} F^{\prime \prime}\left(\gamma_{0}\right)}=e^{F\left(\gamma_{0}\right)} \frac{1}{\sqrt{2 \pi F^{\prime \prime}\left(\gamma_{0}\right)}}
$$

onde $\gamma_{0}$ satisfaz a condição de ponto de sela:

$$
\left.\frac{\partial F(\gamma, r, Y)}{\partial Y}\right|_{\gamma_{0}}=0, \quad \gamma_{0}=\gamma_{0}(r, Y)
$$

Para visualizarmos a solução da equação acima, é útil termos em mente que a função $\chi(\gamma)$, é uma função convexa com seu mínimo em $\gamma=1 / 2$ e pólos simples em $\gamma=0$ e 
$\gamma=1$. Uma boa aproximação para esta função em $0<\gamma<1$ é dada por:

$$
2 \Psi(1)-\Psi(\gamma)-\Psi(1-\gamma) \approx \frac{1}{\gamma}+\frac{1}{1-\gamma}+4 \ln 2-4
$$

Assim, existe um único ponto de sela $\gamma_{0}$ entre $0<\gamma<1$, cuja posição varia entre $0 \mathrm{e}$ 1 dependendo do valor da razão $r / \bar{\alpha}_{s} Y$. Existem três casos limites de interesse:

1. Quando $r / \bar{\alpha}_{s} Y$ é positivo e suficientemente grande: o ponto de sela está próximo de $\gamma=0$.

2. Quando $r / \bar{\alpha}_{s} Y \sim 0$ : o ponto de sela está próximo de $\gamma=1 / 2$.

3. Quando $r / \bar{\alpha}_{s} Y$ é extremamente negativo: o ponto de sela está próximo de $\gamma=1$.

O primeiro caso é relevante quando consideramos a equação BFKL no espaço dos momentos, onde $r \equiv \ln \left(k_{\perp}^{2} / \Lambda^{2}\right)$ é sempre positivo no alcance de interesse. O terceiro caso aplica-se apenas no espaço das coordenadas, onde $r \equiv \ln \left(r_{\perp}^{2} / l^{2}\right)$ é negativo. $\mathrm{O}$ primeiro e terceiro caso correspondem a aproximação de duplo logarítmo (DLA), que descreve o comportamento dominante da solução em grande $k_{\perp}^{2}$ (ou pequeno $r_{\perp}^{2}$ ) para $Y$ fixo e grande; este limite é um limite comum às equações BFKL e DGLAP. Por outro lado, o segundo caso, aplica-se aos espaços dos momentos $(r>0)$ e das coordenadas $(r<0)$.

No primeiro caso, quando $\gamma_{0} \ll 1$, temos

$$
F(\gamma) \simeq r \gamma+\frac{\bar{\alpha}_{s} Y}{\gamma}
$$

Usando a condição de ponto de sela, obtemos o seguinte resultado para o ponto de sela:

$$
\gamma_{0}=\sqrt{\frac{\bar{\alpha}_{s} Y}{r}} .
$$


Inserindo esse resultado na equação $(3,3.29)$, obtemos o seguinte resultado para $N$ :

$$
N\left(k_{\perp}\right) \simeq e^{2 \sqrt{\bar{\alpha}_{s} Y r}} \sqrt{\frac{\gamma^{3}}{4 \pi \bar{\alpha}_{s} Y}},
$$

onde $r=\ln \left(k_{\perp}^{2} / \Lambda^{2}\right)$. Esta solução é conhecida como a solução DLA no espaço dos momentos.

A condição $\gamma_{0} \ll 1$ deve ser reformulada para $\gamma_{0} \ll 1 / 4$, uma vez que $\gamma_{0}=1 / 4$ é o ponto médio entre os pontos de sela 0 e $1 / 2$. Esse critério implicaria na seguinte condição de validade da solução DLA $(3,3.32)$ no espaço dos momentos: $16 \bar{\alpha}_{s} Y \ll$ $\ln \left(k_{\perp}^{2} / \Lambda^{2}\right)$.

No segundo caso quando $\gamma \rightarrow 1 / 2$, temos que o ponto de sela será:

$$
\gamma_{0} \simeq \frac{1}{2}-\delta, \quad \delta \equiv \frac{r}{\beta \bar{\alpha}_{s} Y}
$$

isso implica que

$$
\begin{aligned}
F\left(\gamma_{0}\right) & =r\left(\frac{1}{2}-\delta\right)+\bar{\alpha}_{s} Y \chi(1 / 2-\delta) \\
F^{\prime \prime}\left(\gamma_{0}\right) & =\bar{\alpha}_{s} Y \chi^{\prime \prime}(1 / 2-\delta)
\end{aligned}
$$

onde

$$
\begin{gathered}
\chi(1 / 2)=2 \Psi(1)-2 \Psi(1 / 2)=4 \ln 2 \\
\chi^{\prime \prime}(1 / 2)=-2 \Psi^{\prime \prime}(1 / 2)=28 \zeta(3) \equiv \beta .
\end{gathered}
$$

Inserindo esses resultados em (3,3.29) obtemos:

$$
N \simeq e^{\omega \bar{\alpha}_{s} Y} e^{\frac{1}{2} r} \exp \left\{-\frac{r^{2}}{2 \beta \bar{\alpha}_{s} Y}\right\} \frac{1}{\sqrt{2 \pi \beta \bar{\alpha}_{s} Y}}
$$

onde $\omega=\chi(1 / 2)=4 \ln 2$. 
Quando $r=\ln \left(\frac{k_{\perp}^{2}}{\Lambda^{2}}\right)$, esta equação corresponde à solução usual da equação de BFKL no espaço dos momentos. Por outro lado, quando $r=\ln \left(\frac{r^{2}}{l^{2}}\right)$, ela também é solução da equação BK linearizada no espaço das coordenadas.

$\mathrm{O}$ ponto de sela $\gamma_{0} \simeq 1 / 2-\delta$ permanece próximo de $1 / 2$ para todo $r$ tal que $\delta \ll 1 / 4$. Isso acontece quando: $\ln \left(k_{\perp}^{2} / \Lambda^{2}\right) \ll 8 \bar{\alpha}_{s} Y$.

\subsection{Scaling geométrico na equação de BFKL}

É possível determinar o momento de saturação a partir da solução $N\left(r_{\perp}\right)$ da equação de BFKL, usando a condição de saturação:

$$
N\left(r_{\perp}=1 / Q_{s}(Y)\right)=1
$$

Para obtermos uma estimativa de $Q_{s}(Y)$, iremos usar as aproximações de ponto de sela nas equações $(3,3.29)$ e $(3,3.30)$ e então estudar as propriedades de scaling da solução da equação de BFKL acima da escala de saturação. Para este objetivo, é importante notar que na equação $(3,3.30)$ o ponto de sela é uma função de uma única variável, ou seja, $(3,3.30)$ implica que:

$$
\begin{aligned}
& r+\left.\bar{\alpha}_{s} Y\left[-\Psi^{\prime}(\gamma)-\Psi^{\prime}(1-\gamma)\right]\right|_{\gamma_{0}}=0 \\
& \left.\frac{\partial}{\partial \gamma}[\Psi(\gamma)+\Psi(1-\gamma)]\right|_{\gamma_{0}}=\frac{r}{\bar{\alpha}_{s} Y} \equiv-\mathcal{R}
\end{aligned}
$$

$\log 0$

$$
\gamma_{0}(r, Y)=\gamma_{0}(\mathcal{R})
$$

Se estimamos a integral de Mellin apenas pelo ponto de sela, obtemos

$$
N\left(r_{\perp}\right) \simeq e^{\bar{\alpha}_{s} Y F\left(\gamma_{0}(\mathcal{R}), \mathcal{R}\right)}
$$

onde, quando comparamos com as equações $(3,3.29)$ e $(3,3.30)$, mudamos a definição 
de $F(\gamma, r, Y)$ colocando em evidência o fator $\bar{\alpha}_{s} Y$. O critério de saturação dá a seguinte condição para $F$ :

$$
F\left(\gamma_{0}(\mathcal{R}), \mathcal{R}\right)=0
$$

para

$$
\mathcal{R}=\mathcal{R}_{s}=-\frac{r_{s}}{\bar{\alpha}_{s} Y}=\frac{1}{\bar{\alpha}_{s} Y} \ln \frac{1}{r_{\perp, s}^{2} \Lambda^{2}}=\frac{1}{\bar{\alpha}_{s} Y} \ln \frac{Q_{s}^{2}}{\Lambda^{2}}
$$

que é uma equação para $\mathcal{R}_{s}$ e finalmente para $Q_{s}(Y)$.

A solução $\mathcal{R}_{s}$ para $F\left(\gamma_{0}(\mathcal{R}), \mathcal{R}\right)=0$ é um número puro, $\mathcal{R}_{s} \equiv c$, e não uma função de $Y$. Isso pode ser verificado como segue.

Dada a equação

$$
\left.\frac{\partial}{\partial \gamma}[\Psi(\gamma)+\Psi(1-\gamma)]\right|_{\gamma_{0}}=-\mathcal{R}
$$

podemos deduzir uma equação para um valor particular $\gamma_{s} \equiv \gamma_{0}\left(\mathcal{R}_{s}\right)$ tal que o ponto de sela na saturação fica:

$$
\left.\frac{\partial}{\partial \gamma}[\Psi(\gamma)+\Psi(1-\gamma)]\right|_{\gamma_{s}}=-\mathcal{R}_{s}
$$

Multiplicando ambos os lados da equação acima por $\gamma_{s}$ temos:

$$
-\left.\gamma_{s} \frac{\partial}{\partial \gamma}[\Psi(\gamma)+\Psi(1-\gamma)]\right|_{\gamma_{s}}=\gamma_{s} \mathcal{R}_{s}
$$

onde

$$
\gamma_{s} \mathcal{R}_{s}=\gamma_{s}\left(-\frac{r_{s}}{\bar{\alpha}_{s} Y}\right)
$$

Como o $\left.F\left(\gamma_{0}(\mathcal{R}), \mathcal{R}\right)\right|_{\mathcal{R}=\mathcal{R}_{s}}=0$, temos:

$$
r_{s} \gamma_{s}+\bar{\alpha}_{s} Y \chi\left(\gamma_{s}\right)=0
$$

e portanto

$$
\chi\left(\gamma_{s}\right)=-\frac{r_{s} \gamma_{s}}{\bar{\alpha}_{s} Y}
$$


Temos também

$$
\gamma_{s} \mathcal{R}_{s}=\chi\left(\gamma_{s}\right)=2 \Psi(1)-\Psi\left(\gamma_{s}\right)-\Psi\left(1-\gamma_{s}\right)
$$

e portanto:

$$
-\left.\gamma_{s} \frac{\partial}{\partial \gamma}[\Psi(\gamma)+\Psi(1-\gamma)]\right|_{\gamma_{s}}=\chi\left(\gamma_{s}\right)
$$

ou seja,

$$
\chi^{\prime}\left(\gamma_{s}\right)=\frac{\chi\left(\gamma_{s}\right)}{\gamma_{s}} .
$$

Resolvendo numericamente essa equação, encontra-se [46]: $\mathcal{R}_{s} \simeq 4.88 \mathrm{e}$

$$
\gamma_{s} \simeq 0.63
$$

Agora vamos resolver a equação $(3,4.36)$ para $\mathcal{R}$ um pouco acima de $\mathcal{R}_{s}$, ou seja, para distâncias $r_{\perp}$ que, ainda sendo muito mais curtas do que o comprimento de saturação $1 / Q_{s}(Y)$, estão próximas em unidades logarítmicas. Precisamos que

$$
0<\mathcal{R}-\mathcal{R}_{s} \ll \mathcal{R}_{s}, \text { ou } 1<\ln \frac{Q^{2}}{Q_{s}^{2}(Y)} \ll \ln \frac{Q_{s}^{2}(Y)}{\Lambda^{2}}
$$

A condição $\mathcal{R}>\mathcal{R}_{s}$ (ou $Q^{2} \gg Q_{s}^{2}(Y)$ ) assegura que estamos no regime linear. A condição $\mathcal{R}-\mathcal{R}_{s} \ll \mathcal{R}_{s}$ permite-nos estudar a aproximação de $N\left(r_{\perp}\right)$ na direção da saturação numa expansão limitada em potências de $\mathcal{R}-\mathcal{R}_{s}$. Em ordem linear temos para essa expansão

$$
\begin{aligned}
F\left(\gamma_{0}(\mathcal{R}), \mathcal{R}\right) & \simeq F\left(\gamma_{0}\left(\mathcal{R}_{s}\right), \mathcal{R}_{s}\right)+\left.\frac{d}{d \mathcal{R}} F\left(\gamma_{0}(\mathcal{R}), \mathcal{R}\right)\right|_{\mathcal{R}=\mathcal{R}_{s}}+\ldots \\
& =-\gamma_{s}\left(\mathcal{R}-\mathcal{R}_{s}\right)+\ldots
\end{aligned}
$$

Portanto, acima da escala de saturação, a amplitude de espalhamento dipolo-hádron é

$$
N\left(r_{\perp}\right) \simeq \kappa e^{-\bar{\alpha}_{s} Y \gamma_{s}\left(\mathcal{R}-\mathcal{R}_{s}\right)}
$$


Uma vez que

$$
\bar{\alpha}_{s} Y\left(\mathcal{R}-\mathcal{R}_{s}\right)=\ln \frac{1}{r_{\perp}^{2} Q_{s}^{2}(Y)}
$$

podemos escrever

$$
N\left(r_{\perp}\right) \simeq \kappa\left(r_{\perp}^{2} Q_{s}^{2}(Y)\right)^{\gamma_{s}}
$$

para $\kappa<1$. Vemos que esta equação mostra o scaling geométrico com a dimensão anômala

$$
\gamma=1-\gamma_{s} \simeq 0.37
$$

determinada pelo ponto de sela $\gamma_{s} \equiv \gamma_{0}\left(\mathcal{R}_{s}\right)$ na saturação. Observamos que dentro da região $Q_{s}^{2} \leq Q^{2} \leq Q_{s}^{4} / \Lambda^{2}$, a equação BFKL têm uma solução que prediz o scaling geométrico.

Incluindo termos de segunda ordem na expansão $(3,4.43)$, a equação para $N\left(r_{\perp}\right)$ fica [46]:

$$
N\left(r_{\perp}\right) \simeq \kappa\left(r_{\perp}^{2} Q_{s}^{2}(Y)\right)^{\gamma_{s}} \exp \left[-\frac{\gamma_{s}^{\prime}}{2 \bar{\alpha}_{s} Y}\left(\ln \frac{1}{r_{\perp}^{2} Q_{s}^{2}(Y)}\right)^{2}\right]
$$

onde definimos $\gamma_{s}^{\prime} \equiv \frac{d \gamma_{0}(\mathcal{R})}{d \mathcal{R}} \mathcal{R}_{s}$

\subsection{Modelos fenomenológicos}

A seção de choque de dipolo $\sigma_{d i p}$ contém todas as informações sobre o alvo e sobre a física das interações fortes. No formalismo do condensado de vidros coloridos (CGC) $[47,48,49], \sigma_{d i p}$ pode ser calculado na aproximação eikonal e é dada por:

$$
\sigma_{d i p}(x, \boldsymbol{r})=2 \int d^{2} \boldsymbol{b} \mathcal{N}(x, \boldsymbol{r}, \boldsymbol{b})
$$

onde $\mathcal{N}$ é a amplitude de espalhamento dipolo-alvo (para um dado parâmetro de impacto b), que carrega toda a informação sobre o espalhamento hadrônico, e assim sobre os efeitos não-lineares na função de onda hadrônica. Como foi visto nas seções anteri- 
ores, a função $\mathcal{N}$ pode ser obtida resolvendo-se uma equação de evolução apropriada em rapidez $Y \equiv \ln (1 / x)$. As principais propriedades de $\mathcal{N}$ são:

1. para pequenos dipolos $\left(\boldsymbol{r} \ll 1 / Q_{\mathrm{s}}\right), \mathcal{N}(\boldsymbol{r}) \approx \boldsymbol{r}^{2}$, o que implica que o sistema é fracamente interagente;

2. para grandes dipolos $\left(\boldsymbol{r} \gg 1 / Q_{\mathrm{s}}\right)$, o sistema é fortemente absorvedor e portanto $\mathcal{N}(\boldsymbol{r}) \approx 1$. Esta propriedade está associada à grande densidade de glúons saturados na função de onda hadrônica.

É útil assumir que a dependência no parâmetro de impacto de $\mathcal{N}$ possa ser fatorizada como

$$
\mathcal{N}(x, \boldsymbol{r}, \boldsymbol{b})=\mathcal{N}(x, \boldsymbol{r}) S(\boldsymbol{b})
$$

tal que

$$
\sigma_{d i p}(x, \boldsymbol{r})=\sigma_{0} \mathcal{N}(x, \boldsymbol{r})
$$

com $\sigma_{0}$ sendo um parâmetro livre relacionado à QCD não-perturbativa.

Muitos modelos para a seção de choque de dipolo têm sido usado na literatura para descrever os dados de HERA [26, 50]. Em [26] Golec-Biernart e Wüsthoff (GBW) propuseram o seguinte modelo fenomenológico de saturação:

$$
\mathcal{N}(x, \boldsymbol{r})=\left[1-\exp \left(-\frac{\left(Q_{\mathrm{s}}(x) \boldsymbol{r}\right)^{2}}{4}\right)\right]
$$

com

$$
Q_{s}^{2}=Q_{0}^{2} e^{\lambda \ln \left(x_{0} / x\right)}
$$

Os parâmetros $\sigma_{0}, \lambda$ e $x_{0}$, foram ajustados aos dados de HERA obtendo-se $\sigma_{0}=$ $23.03(29.12) \mathrm{mb}, \lambda=0.288(0.277)$ e $x_{0}=3.04 \cdot 10^{-4}\left(3.41 \cdot 10^{-4}\right)$ para uma análise de 3-sabores (4-sabores) [26]. Um parâmetro adicional é a massa efetiva do quark leve, $m_{f}=0.14 \mathrm{GeV}$. Deve ser observado que a massa do quark funciona como um regulador da seção de choque de fotoprodução $\left(Q^{2}=0\right)$. A massa do quark leve é um dos inputs não-perturbativos do modelo. A massa do quark charme é $m_{c}=1.5 \mathrm{GeV}$. 
Uma transição suave para o limite de fotoprodução é obtida com uma modificação da variável de Bjorken como

$$
\tilde{x}=x\left(1+\frac{4 m_{f}^{2}}{Q^{2}}\right)=\frac{Q^{2}+4 m_{f}^{2}}{W^{2}}
$$

Observando a equação $(3,5.50)$ notamos que quando $Q_{s}^{2}(x) \boldsymbol{r}^{2} \ll 1$, o modelo reduzse à transparência de cor; e quando nos aproximamos da região $Q_{s}^{2}(x) \boldsymbol{r}^{2} \geq 1$, a exponencial assume valores muito menores do que um. Intuitivamente isto é o que acontece quando o próton começa parecer negro. Embora a parametrização GBW dê uma boa descrição dos dados antigos de HERA, isso não ocorre para os novos dados, que têm uma precisão maior. Esta limitação vem do fato de que este modelo falha ao descrever a violação de scaling de Bjorken, e sua forma funcional vem apenas de uma aproximação da QCD não-linear. No que se segue vamos chamar esse modelo de GBW.

Outro modelo inspirado no CGC foi proposto na referência [50] para descrever os dados de HERA. Este modelo está baseado na aproximação da BFKL na fronteira do regime de saturação. Em particular a amplitude de espalhamento frontal, foi calculada nas aproximações da BFKL em ordem dominante (LO) e próxima ordem dominante (NLO), na região de scaling geométrico [51]. Assim o ponto de partida é a equação $(3,4.46)$

$$
\mathcal{N}(x, \boldsymbol{r})=\left[\boldsymbol{r}^{2} Q_{s}^{2}(x)\right]^{\gamma_{s}} \exp \left[-\frac{\ln ^{2}\left(\boldsymbol{r}^{2} Q_{s}^{2}\right)}{2 \beta \bar{\alpha}_{s} Y}\right]
$$

onde a potência $\gamma_{s}$ é o ponto de sela (BFKL) na vizinhança da linha de saturação $Q^{2}=Q_{s}^{2}(x)$ e $\beta=1 / \gamma_{s}^{\prime}$. Neste modelo a normalização da seção de choque de dipolo é dada por $\sigma_{0}=2 \pi R_{p}^{2}$, onde $R_{p}$ é o raio do próton. Além disso, a dimensão anômala é definida por $\gamma=1-\gamma_{s}$. Como usual no formalismo de BFKL, $\bar{\alpha}_{s}=N_{c} \alpha_{s} / \pi$ e $\beta \simeq 28 \zeta(3)$. O fator de difusão quadrático no expoente dá o aumento da violação de scaling, o qual é essencial para descrever os dados de HERA. Como a amplitude de 
espalhamento frontal na equação $(3,5.53)$ não inclui uma extrapolação da região de scaling geométrico para região de saturação, os autores da referência [50] construíram uma parametrização para $\mathcal{N}(x, \boldsymbol{r})$, a qual interpola suavemente entre os limites de grande e pequeno dipolo: a solução da equação de BFKL para pequenos dipolos, $\boldsymbol{r} \ll 1 / Q_{s}(x)$, e a Lei de Levin-Tuchin [45] para grandes dipolos, $\boldsymbol{r} \gg 1 / Q_{s}(x)$. Um ajuste à função de estrutura $F_{2}\left(x, Q^{2}\right)$ foi feito no alcance cinemático de interesse, mostrando que este ajuste não é tão sensível aos detalhes da interpolação (para uma análise fenomenológica dos resultados de HERA usando soluções numéricas da equação BK, veja [52]). A amplitude de espalhamento frontal dipolo-alvo foi parametrizada por:

$$
\mathcal{N}(x, \boldsymbol{r})= \begin{cases}\mathcal{N}_{0}\left(\frac{\boldsymbol{r} Q_{s}}{2}\right)^{2\left(\gamma_{s}+\frac{\ln \left(2 / \boldsymbol{r}_{\left.Q_{s}\right)}\right.}{\kappa \lambda Y}\right)}, & \text { para } \boldsymbol{r} Q_{s}(x) \leq 2 \\ 1-\exp ^{-a \ln ^{2}\left(b \boldsymbol{r} Q_{s}\right)}, & \text { para } \boldsymbol{r} Q_{s}(x)>2\end{cases}
$$

onde a expressão para $\boldsymbol{r} Q_{s}(x)>2$ (região de saturação) tem a forma funcional correta, que foi obtida resolvendo-se a equação BK (equação $(3,2.22)$. No que segue chamaremos o modelo acima de IIM. Os coeficientes $a$ e $b$ são determinados a partir da condição de continuidade da seção de choque de dipolo em $\boldsymbol{r} Q_{s}(x)=2$. Os coeficientes $\gamma_{s}=0.63$ e $\kappa=9.9$ são fixados pela solução LO BFKL. Em nosso cálculos usaremos os parâmetros $R_{p}=0.641 \mathrm{fm}, \lambda=0.253, x_{0}=0.267 \times 10^{-4}$ e $\mathcal{N}_{0}=0.7$, os quais dão o melhor resultado para o ajuste do dados de HERA. Recentemente este modelo foi usado em estudos fenomenológicos da produção de mésons vetorias [53] e em processos difrativos em HERA [36], bem como para a descrição da função de estrutura longitudinal [54].

Kharzeev, Kovchegov e Tuchin (KKT) propuseram uma nova parametrização para a amplitude de espalhamento de dipolo, desta vez para descrever a produção de hádrons em colisões $d A u$ [55]. Os autores propuseram uma parametrização para a amplitude de espalhamento inspirada nas soluções analíticas aproximadas da equação BK para os regimes de saturação e transparência de cor. Neste modelo a amplitude de 
espalhamento frontal dipolo-alvo é dada por [55]

$$
\mathcal{N}(\boldsymbol{r}, x)=1-\exp \left[-\frac{1}{4}\left(\boldsymbol{r}^{2} \frac{C_{F}}{N_{c}} Q_{s}^{2}\right)^{\gamma\left(Y, \boldsymbol{r}^{2}\right)}\right]
$$

onde a dimensão anômala $\gamma\left(Y, \boldsymbol{r}^{2}\right)$ é

$$
\gamma\left(Y, \boldsymbol{r}^{2}\right)=\frac{1}{2}\left(1+\frac{\xi\left(Y, \boldsymbol{r}^{2}\right)}{\xi\left(Y, \boldsymbol{r}^{2}\right)+\sqrt{2 \xi\left(Y, \boldsymbol{r}^{2}\right)}+7 \zeta(3) c}\right),
$$

com $c$ um parâmetro livre e

$$
\xi\left(Y, \boldsymbol{r}^{2}\right)=\frac{\ln \left[1 /\left(\boldsymbol{r}^{2} Q_{s 0}^{2}\right)\right]}{(\lambda / 2)\left(Y-Y_{0}\right)}
$$

Os autores assumem que a escala de saturação pode ser expressa por

$$
Q_{s}^{2}(Y)=\Lambda^{2} A^{1 / 3}\left(\frac{1}{x}\right)^{\lambda}
$$

A forma da dimensão anômala é inspirada pela solução analítica da equação de BFKL. Ou seja, no limite $\boldsymbol{r} \rightarrow 0$ com $Y$ fixo, voltamos a dimensão anômala na aproximação de duplo logaritmo $\gamma \approx 1-\sqrt{1 /(2 \xi)}$. No limite de grande $Y$ para $\boldsymbol{r}$ fixo, a equação $(3,5.56)$ reduz-se à expressão da dimensão anômala na vizinhança do ponto de sela na aproximação de logaritmo dominante $\gamma \approx \frac{1}{2}+\frac{\xi}{14 c \zeta(3)}$, como pode ser visto a partir da equação (3,3.33). Nos cálculos de [55] assume-se que o valor característico de $\boldsymbol{r}$ é $\boldsymbol{r} \approx 1 /\left(2 k_{T}\right)$ onde $k_{T}$ é o momento transversal do quark de valência, e $\gamma$ foi aproximado por $\gamma\left(Y, \boldsymbol{r}^{2}\right) \approx \gamma\left(Y, 1 /\left(4 k_{T}^{2}\right)\right)$. Na expressão acima os parâmetros $\Lambda=0.6 \mathrm{GeV}$ e $\lambda=0.3$ são fixados pelos dados do DIS [26]. A escala de saturação inicial, usada em $(3,5.57)$ é definida por $Q_{s 0}^{2}=Q_{s}^{2}\left(Y_{0}\right)$ e é um parâmetro. Quando aplicada para descrever os dados de RHIC, a amplitude $\mathcal{N}(\boldsymbol{r}, x)$ deve ser convoluída com a função de distribuição de quarks no hádron e com a função de fragmentação do quark. Além disso, a contribuição gluônica deve ser adicionada. Esses procedimentos introduzem 
incertezas nas predições, que podem ser estimados apenas se o formalismo for aplicado a outros processos.

O principal objetivo das parametrizações IIM e KKT é simular a física do CGC em toda a região cinemática. Temos apenas um controle teórico sob os regimes assintóticos de saturação e transparência de cor. Assim devemos escolher um Ansatz de interpolação para obter uma parametrização que possa ser usada em cálculos práticos. Além disso, embora ambas as parametrizações tenham uma forma semelhante, a parametrização KKT, em contraste à IIM, inclui o limite de duplo logaritmo tanto quanto o comportamentto correto nas regiões de saturação e scaling geométrico. Este limite é importante para grande momento transversal, permitindo-nos conectar a física do CGC com as previsões da evolução DGLAP. Entretanto, determinar qual é o limite linear correto (BFKL/DGLAP) na região cinemática do HERA e RHIC é ainda uma questão em aberto.

$\mathrm{Na}$ referência [56] outro modelo fenomenológico de saturação foi proposto para descrever os dados de colisões $d A u$ no RHIC. No que segue esse modelo será chamado de modelo DHJ. A modificação básica desse modelo com respeito ao modelo KKT é a parametrização da dimensão anômala que agora é dada por

$$
\gamma\left(Y, \boldsymbol{r}^{2}\right)=\gamma_{s}+\Delta \gamma\left(Y, \boldsymbol{r}^{2}\right)
$$

onde

$$
\Delta \gamma\left(Y, \boldsymbol{r}^{2}\right)=\left(1-\gamma_{s}\right) \frac{\left|\log \frac{1}{\boldsymbol{r}^{2} Q_{T}^{2}}\right|}{\lambda Y+\left|\log \frac{1}{\boldsymbol{r}^{2} Q_{T}^{2}}\right|+d \sqrt{Y}},
$$

$\operatorname{com} Q_{T}=Q_{s}(Y)$ sendo uma escala típica dura do processo, $\lambda=0.3$ e $d=1.2$. Além disso, $\gamma_{s}=0.63$ é a dimensão anômala para a evolução BFKL com as condições de contorno da saturação.

No próximo capítulo iremos analisar estes modelos em maior detalhe, comparando suas predições para HERA e RHIC. Em particular, demonstraremos que estas parametrizações não descrevem simultaneamente ambos os conjuntos de dados. 
Como um comentário adicional, vale dizer que existem modelos na literatura que não fazem uso da física de saturação e que, entretanto, conseguem descrever os dados de HERA inclusivos e difrativos. Um dos modelos mais conhecido é o chamado modelo de duas componentes [57]. Esse modelo assume que a seção de choque total pode ser escrita como uma componente soft e uma hard. 


\section{Capítulo 4}

\section{Universalidade dos modelos fenomenológicos}

No capítulo anterior demonstramos para o caso do núcleon que a escala de saturação $Q_{s}$ que determina a escala abaixo da qual os efeitos de saturação não podem ser desprezados, cresce com a energia. Generalizando para o caso do núcleo temos que a escala de saturação será dada por

$$
Q_{s}^{2}=Q_{s_{0}}^{2} A^{1 / 3}\left(\frac{1}{x}\right)^{\lambda}
$$

onde $\lambda=0.3$ [26] é um parâmetro vinculado aos dados ep no HERA. Na figura 4.1 fazemos um gráfico do plano $A^{1 / 3}$ versus $x$, onde as linhas representam escala de saturação constante. Esta figura mostra como o núcleo pode ser eficiente para ampliar a densidade de gluons e fazer com que o regime de saturação seja atingido em energias mais baixas (maiores valores de $x$ ) do que em colisões contra um núcleon.

Em particular, a escala típica de saturação nas colisões $d A u$ no RHIC é da mesma ordem de grandeza que a escala de saturação típica das colisões no HERA. Isto nos leva a relacionar esses experimentos e ganhar um melhor entendimento da física do CGC em experimentos de altas energias. Em particular podemos verificar a propriedade predita pela física do CGC. 


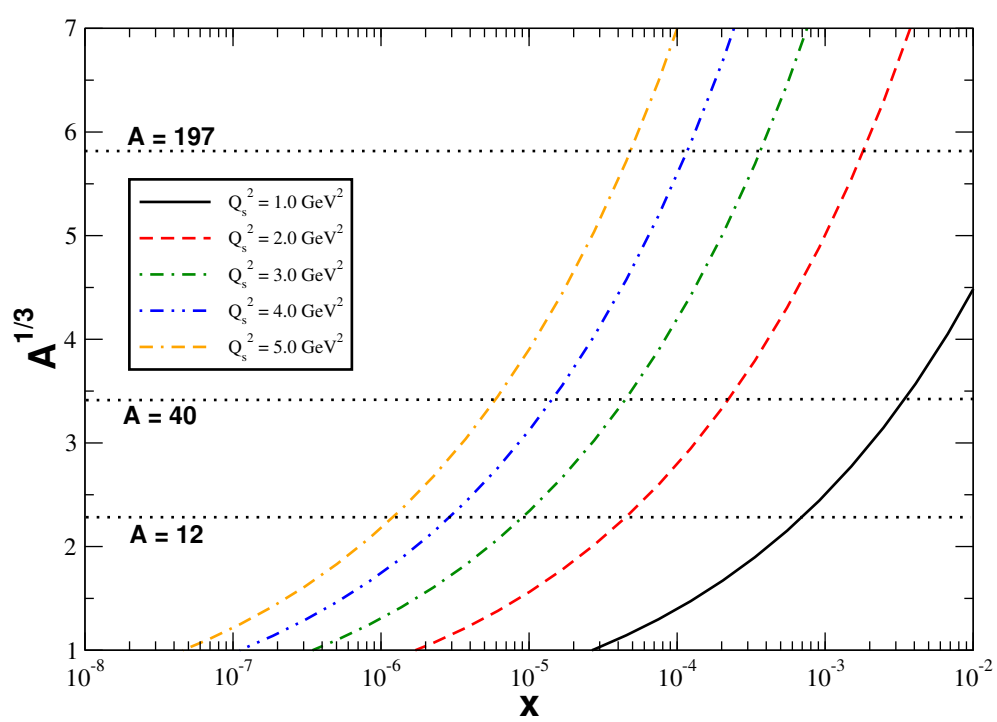

Figura 4.1: Escala de saturação para diferentes valores de $A$ e $x$.

Os resultados apresentados neste capítulo foram publicados nas referências [7, 8].

\subsection{Comparação entre os modelos fenomenológicos}

Nesta seção apresentamos uma comparação detalhada entre as previsões das diferentes parametrizações da amplitude de espalhamento de dipolo e também uma comparação destas previsões com os dados de HERA. Na tabela 4.1 mostramos os limites dos vários $\mathcal{N}$ nos regimes linear $r \ll r_{\text {sat }}$ e de saturação $r \gg r_{\text {sat }}$, onde $r_{\text {sat }} \equiv 1 / Q_{s}$. Como discutido no Capítulo 1, a linha crítica dividindo os regimes denso e diluído é definida pela escala de saturação $Q_{s}$, com a propriedade de que para $x$ menor, o sistema fica mais denso e os pártons começam a interagir entre si. A característica básica dos modelos GBW, IIM, e KKT é que para um dado $r$ esses modelos prevêem que a amplitude tende à unidade em pequenos valores de $x$ em contraste com a solução linear, a qual prediz um crescimento exponencial nesta região cinemática. Outra característica desses modelos é que todos predizem que o sistema sature prematuramente (ou seja, para valores de $x$ grande) quando o tamanho do dipolo é maior. As três parametrizações apresentam a forma funcional semelhante para os dois limites, sendo que 


\begin{tabular}{||c|c|c||}
\hline \hline & $r \ll r_{\text {sat }}$ & $r \gg r_{\text {sat }}$ \\
\hline \hline GBW & $\frac{\left(r Q_{s}\right)^{2}}{4}$ & 1 \\
\hline IIM & $\mathcal{N}_{0}\left(\frac{r Q_{s}}{2}\right)^{2\left(\gamma_{s}+\frac{\ln \left(2 / r Q_{s}\right)}{\kappa \lambda Y}\right)}$ & $1-e^{-a \ln ^{2}\left(b r Q_{s}\right)}$ \\
\hline KKT & $\frac{1}{4}\left(\frac{C_{F}}{N_{c}}\left(r Q_{s}\right)^{2}\right)^{\gamma\left(Y, r^{2}\right)}$ & 1 \\
\hline \hline
\end{tabular}

Tabela 4.1: Limites assintóticos para a amplitude de espalhamento dipolo-alvo em diferentes modelos.

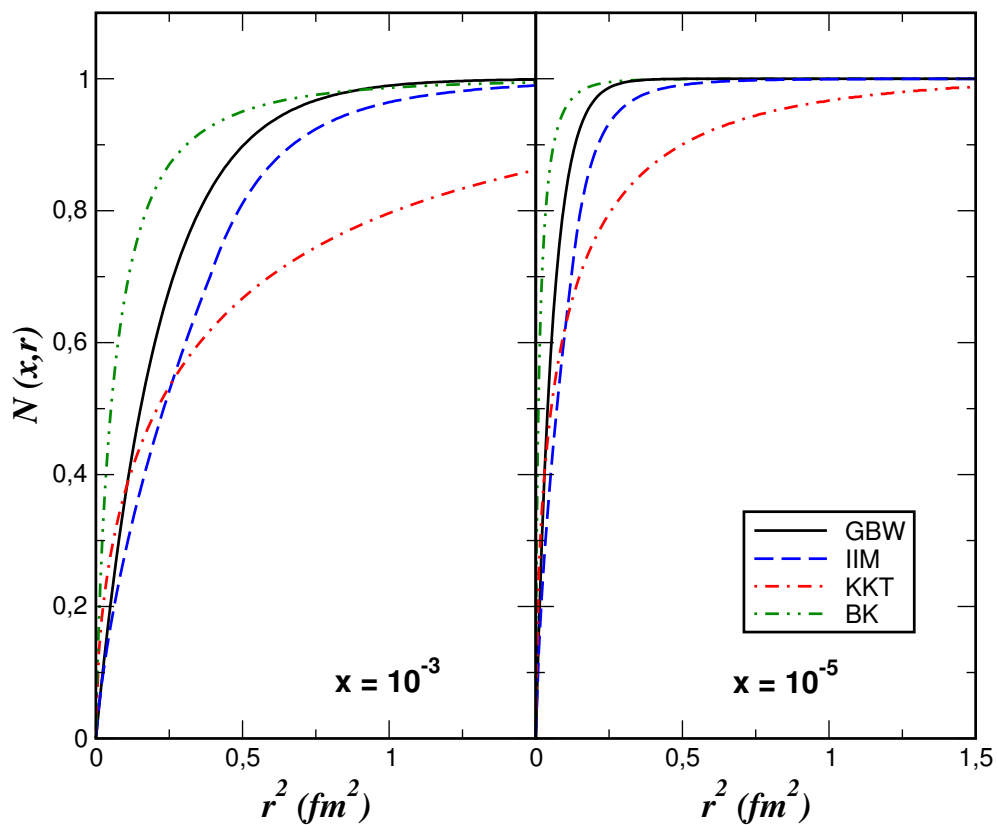

Figura 4.2: Dependência da amplitude de espalhamento de dipolo com o quadrado da separação do par $\left(r^{2}\right)$ para diferentes valores de $x$.

IIM apresenta uma dependência residual em $r Q_{s}$ na região de saturação, mas também mostrando saturação para grandes valores de $r Q_{s}$.

Na figura 4.2 analisamos a dependência da amplitude de espalhamento de dipolo com a separação do par para diferentes valores de $x$. Como esperado da discussão anterior, vemos que enquanto as parametrizações GBW e IIM apresentam um comportamento semelhante para pequenos $r^{2}$, a KKT prediz uma dependência mais suave. No outro limite, as parametrizações GBW e IIM saturam para grandes separações do par, enquanto que KKT ainda apresenta uma dependência residual, mostrando que o regime assintótico é apenas atingido para separações do par muito grandes. A principal característica, que é evidente nos modelos GBW e IIM é que a seção de choque de di- 


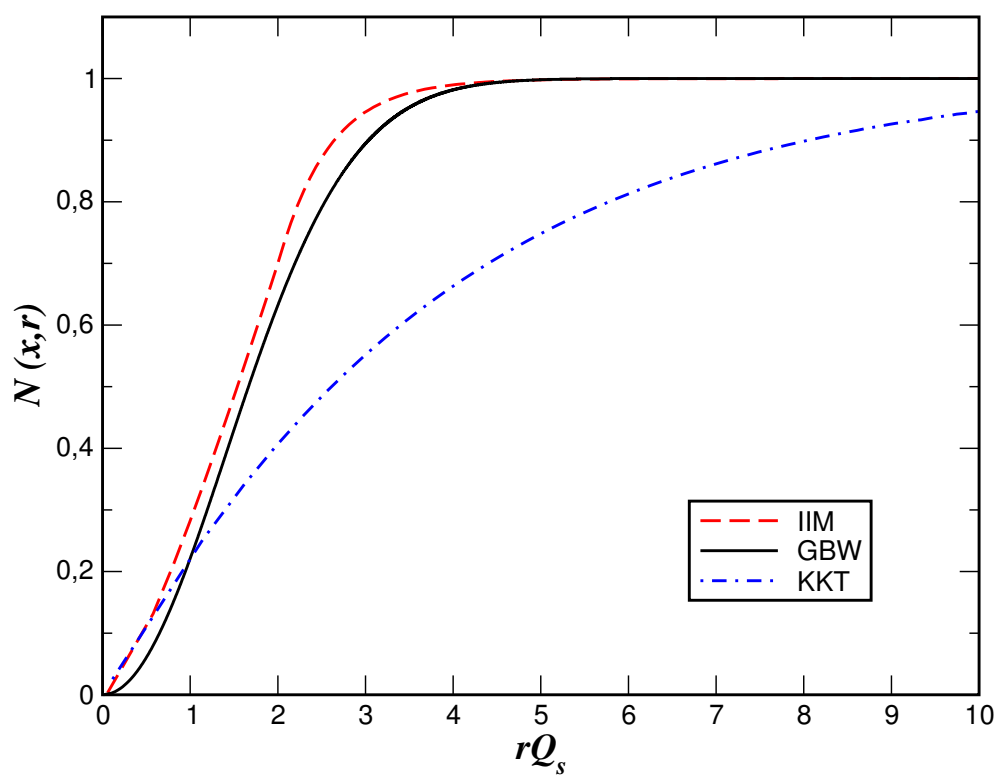

Figura 4.3: A amplitude de espalhamento de dipolo como uma função da variável de scaling $r Q_{s}$.

polo satura para dipolos menores quando $x$ assume valores pequenos. Um importante aspecto a ser enfatizado é a grande diferença entre as previsões na região de transição, que é acessível no HERA. Para comparação também apresentamos as previsões para $\mathcal{N}$ da solução numérica da equação BK obtida em [52]. Vemos que essa solução tem, como esperado, os limites de transparência de cor e saturação para pequeno e grande $r$, respectivamente. É importante enfatizar a grande diferença entre esses resultados e a parametrização KKT. No que segue vamos restringir nossa análise às parametrizações fenomenológicas, que têm seus parâmetros fixados pelos dados experimentais.

Todos esses modelos têm a propriedade de scaling geométrico observado nas soluções da equação BK. Como já foi dito, scaling geométrico significa que a solução da equação BK depende apenas de uma variável combinada $r Q_{s}(x)$, e não de $r$ e $x$ separadamente, ou seja, $\mathcal{N}(r, x) \equiv \mathcal{N}\left(r Q_{s}(x)\right)$. Na figura 4.3, mostramos a dependência da amplitude de espalhamento de dipolo com a variável de scaling $r Q_{s}$. Observamos que as três amplitudes de espalhamento crescem na região de pequenos valores de $r Q_{s}(x)$ como uma potência de $r Q_{s}(x)$, isto é, $\mathcal{N}(r, x) \propto\left(r Q_{s}\right)^{2 \gamma_{e f f}}$. Entretanto, $\gamma_{e f f}$ é diferente para cada modelo, sendo 1 para GBW, $\leq 1$ para IIM e aproximadamente 

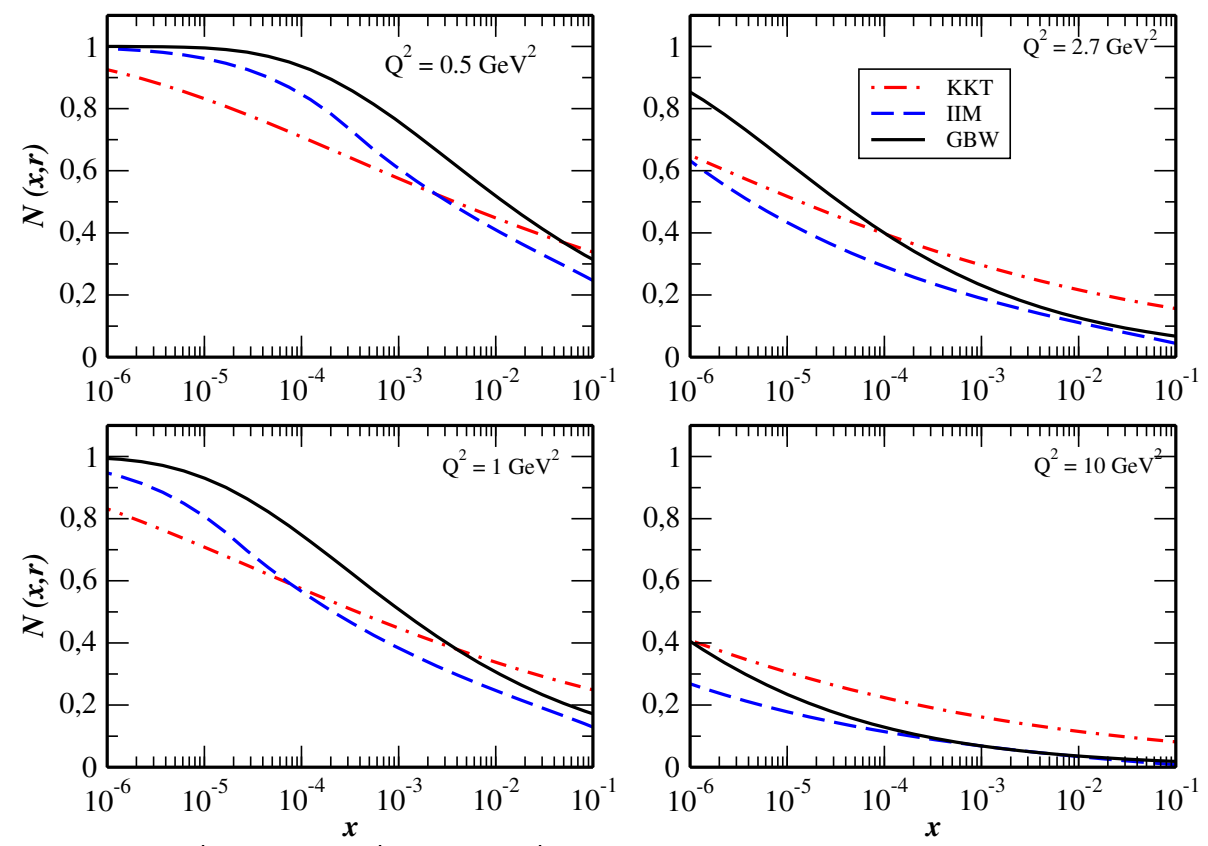

Figura 4.4: Dependência em energia da amplitude de espalhamento de dipolo para diferentes valores da separação quadrada do par $r^{2}=1 / Q^{2}$.

1/2 para KKT. Como a escala de saturação contém a dependência em energia da seção de choque de dipolo, esses modelos apresentam comportamentos distintos com a energia. Isso pode ser visto na figura 4.4, onde apresentamos a dependência em $x$ de $\mathcal{N}(r, x)$ para diferentes valores da separação quadrática do par. Notamos que para grande $Q^{2}$ (pequena separação do par), $\mathcal{N}(r, x)$ é dominada pelo limite linear. Como estes modelos apresentam um comportamento diferente neste limite, a dependência também é diferente, sendo que o modelo GBW apresenta um crescimento mais forte em $x$ pequeno. O comportamento previsto pelo modelo IIM é semelhante ao GBW. Por outro lado o modelo KKT prevê um crescimento mais lento com a energia. Em grande separação do par, $r>r_{s}$ que é típica do regime de saturação, os modelos IIM e GBW predizem saturação da amplitude de espalhamento de dipolo, enquanto que KKT ainda apresenta um crescimento para pequenos valores de $x$. Basicamente nesta parametrização, o regime de saturação assintótico é apenas observado para valores muito pequenos de $x$, além do alcance cinemático de HERA. 


\subsubsection{A função de estrutura do nucleon}

O observável básico medido com grande precisão no HERA é a função de estrutura $F_{2}\left(x, Q^{2}\right)$, que está diretamente relacionada com a seção de choque $\gamma^{*} p$ pela seguinte expressão:

$$
F_{2}\left(x, Q^{2}\right)=\frac{Q^{2}}{4 \pi^{2} \alpha_{e m}}\left(\sigma_{T}^{\gamma^{*} p}+\sigma_{L}^{\gamma^{*} p}\right)
$$

Consequentemente usando a representação de dipolo de cor no DIS, podemos calcular diretamente $F_{2}$ para diferentes parametrizações da amplitude de espalhamento de dipolo. Podemos também estimar a função de estrutura longitudinal

$$
F_{L}\left(x, Q^{2}\right)=Q^{2} /\left(4 \pi^{2} \alpha_{e m}\right) \times \sigma_{L}^{\gamma^{*} p}
$$

Nas figuras 4.5, 4.6 e 4.7 apresentamos uma comparação entre as previsões dos diferentes modelos e os dados de ZEUS [58]. Usamos $\sigma_{d i p}=\sigma_{0} \mathcal{N}$ com $\sigma_{0}$ dado nas parametrizações GBW e IIM. Para a parametrização KKT tratamos $\sigma_{0}$ como um parâmetro livre e fixamos seu valor pelo ajuste aos dados de $F_{2}$ em $Q^{2}=2.7 \mathrm{GeV}^{2}$. Nossa escolha para este valor de virtualidade é justificada pelo fato de que nesta região esperamos que a física de saturação seja dominante. Testamos outras escolhas e verificamos que nossa principal conclusão não é modificada.

Além disso, consideramos duas outras escolhas para a escala típica presente no processo para calcular a função $\gamma\left(Y, r^{2}\right)$ na parametrização KKT. Basicamente, assumimos que $r \approx 1 / Q$, ou $r \approx 1 / Q_{s}$. Como mostraremos, nossas previsões para $F_{2}$ na região cinemática de interesse são quase idênticas. Consideramos apenas poucos valores de virtualidade do fóton na região de baixo $Q^{2}$, onde os efeitos de saturação devem ser importantes. Como esperado, os modelos IIM e GBW descrevem bem os dados experimentais de $F_{2}$. Por outro lado, a parametrização KKT é capaz de descrever os dados para ep apenas numa região cinemática limitada da virtualidade do fóton ao redor da virtualidade onde a normalização foi fixada. O aspecto básico desta parametrização é que ela não é capaz de reproduzir bem a dependência em $Q^{2}$ da função 
de estrutura do próton. Além do mais, essa parametrização prevê uma dependência na energia que é mais lenta do que a observada nos dados. Este comportamento está diretamente relacionado ao comportamento da amplitude de espalhamento de dipolo. A curva denotada por KKTq na figura, representa os resultados obtidos assumindo $r \approx 1 / Q$, enquanto que a curva KKT assume $r \approx 1 / Q_{s}$ no cálculo de $\gamma\left(Y, r^{2}\right)$. Essas duas prescrições diferem apreciavelmente na região de grande $x$ e/ou grande $Q^{2}$.

$\mathrm{Na}$ figura 4.6, temos o mesmo da figura 4.5, mas sendo a normalização das curvas KKT e KKTq fixada para $Q^{2}=10 \mathrm{GeV}^{2}$.

Na figura 4.7, apresentamos as previsões dos diferentes modelos para a função de estrutura longitudinal. Por comparação, também apresentamos as previsões obtidas usando a equação de Altarelli-Martinelli e a parametrização GRV98 para a solução da equação de evolução de DGLAP (para detalhes veja [54]). Neste caso vemos que a parametrização KKT descreve razoavelmente bem os poucos dados existentes [59], como as parametrizações GBW e IIM. Entretanto, esse fato está associado principalmente ao grande erro experimental nos dados existentes. Acreditamos que um futuro estudo experimental da função de estrutura longitudinal seja capaz de discriminar entre essas parametrizações.

Com base na universalidade da função de onda hadrônica prevista pelo formalismo do CGC, esperamos que as parametrizações KKT e DHJ propostas para descrever os dados dAu no RHIC também descrevam os dados de HERA para a função de estrutura do próton na região cinemática onde os efeitos de saturação devem estar presentes $(x$ pequeno e $Q^{2}$ baixo). Porém, esta expectativa falha quando o modelo KKT é aplicado, como verificado acima.

Aqui estendemos a análise para o modelo DHJ sem qualquer modificação dos parâmetros ajustados do RHIC, apenas assumindo $A=1$ e ajustando o parâmetro não-perturbativo $\sigma_{0}$, o qual define a normalização, a fim de descrever os dados experimentais para $F_{2}$ em $Q^{2}=10 \mathrm{GeV}^{2}$. Nas figuras $4.8,4.9,4.10$ e 4.11 apresentamos as previsões do modelo DHJ para a função de estrutura do próton e para a função de estrutura longitudinal, e comparamos com os dados de ZEUS [58]. Vemos que esta 

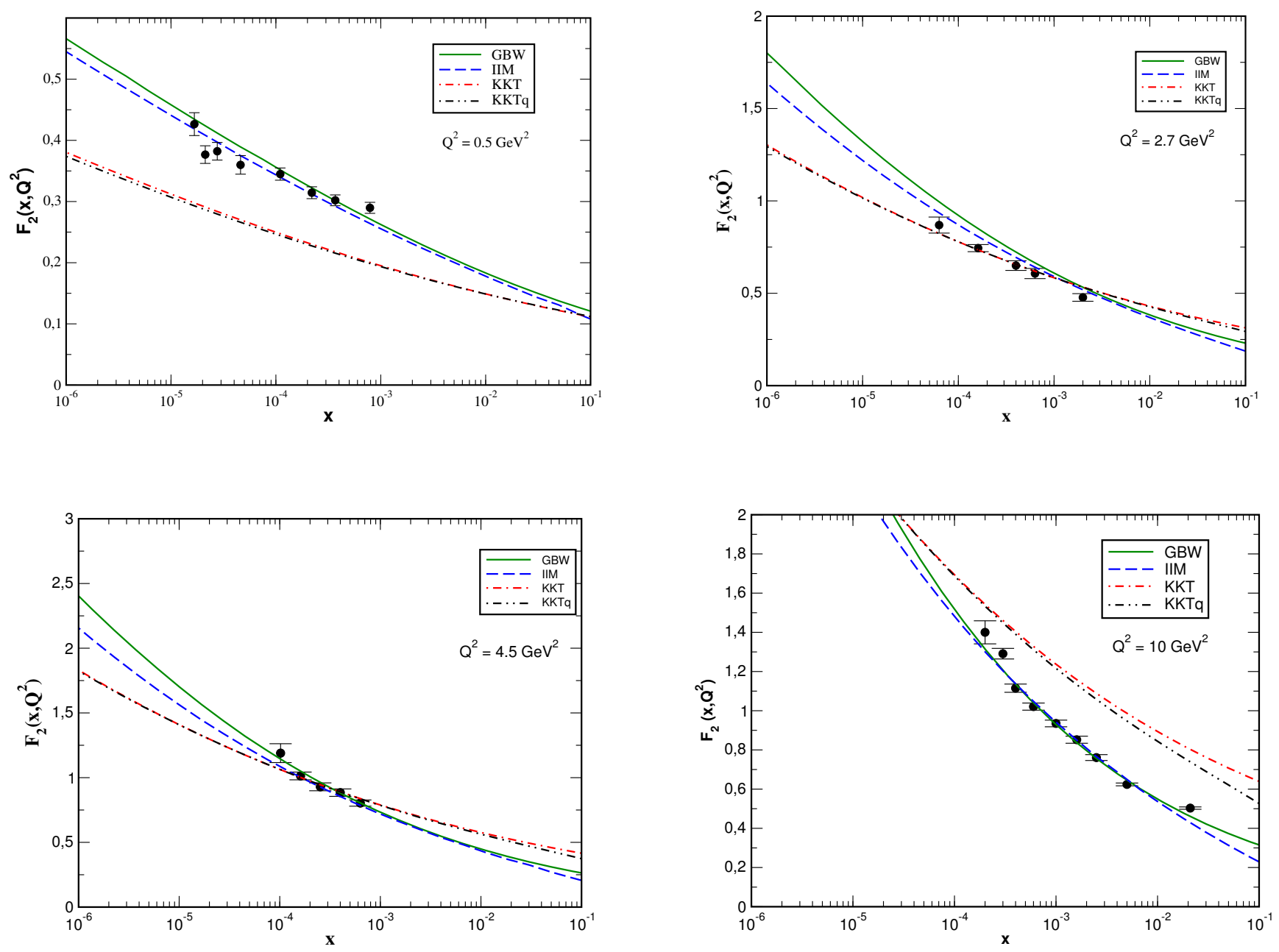

Figura 4.5: Comparação entre as previsões para $F_{2}\left(x, Q^{2}\right)$ em diferentes modelos e para diferentes valores de $Q^{2}$. Os dados são de ZEUS. 

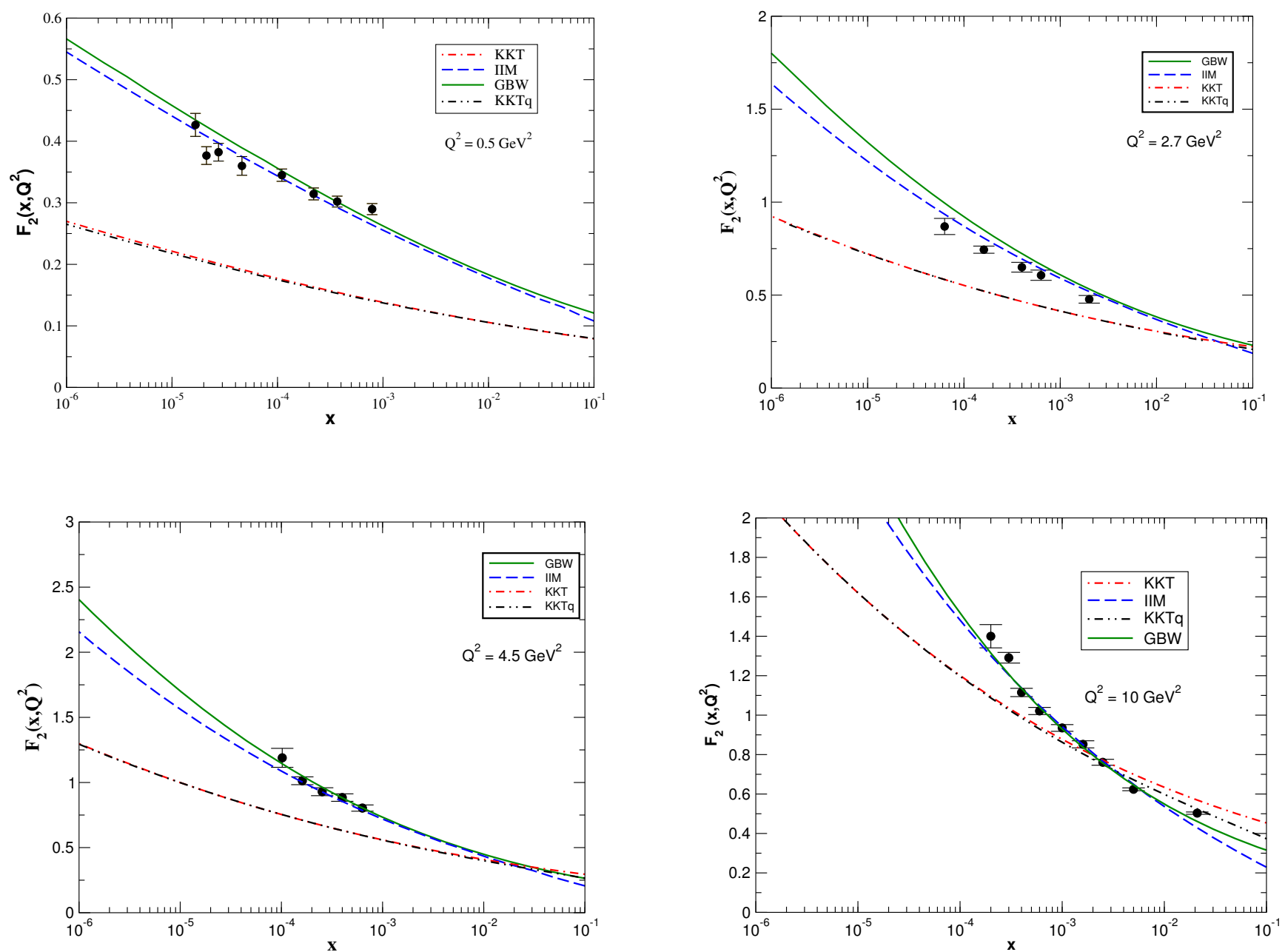

Figura 4.6: Comparação entre as previsões para $F_{2}\left(x, Q^{2}\right)$ em diferentes modelos e para diferentes valores de $Q^{2}$. Os dados são de ZEUS. 

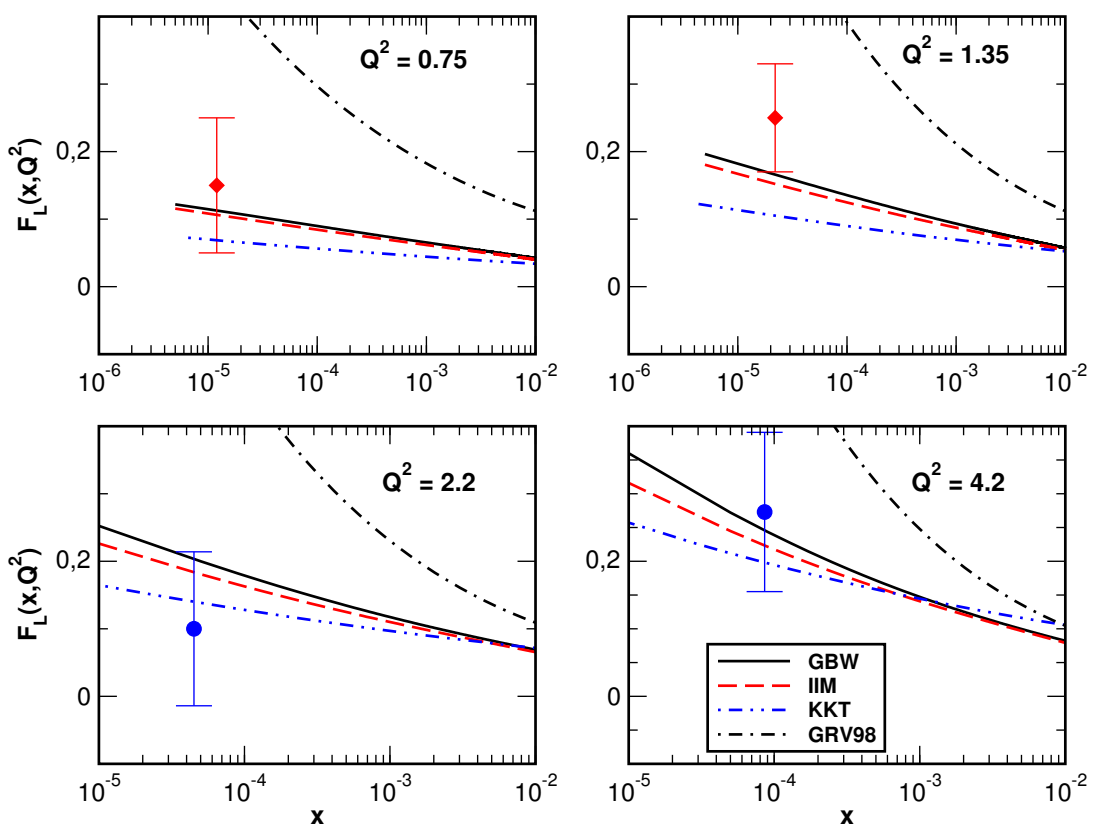

Figura 4.7: Comparação entre as previsões para $F_{L}\left(x, Q^{2}\right)$ em diferentes modelos e para diferentes valores de $Q^{2}$.

parametrização para pequenos e grandes valores de $Q^{2}$ falha ao descrever a evolução em $Q^{2}$ de $F_{2}$ e $F_{L}$. Concluímos que as parametrizações da seção de choque de dipolo que estão vinculadas aos dados do RHIC não são capazes de descrever os dados de HERA.

Uma variação do modelo KKT foi proposta em [60] para descrever os dados da função de estrutura longitudinal no HERA. Essa modificação consistiu na alteração de alguns parâmetros, tais como:

- a escala de saturação ficou definida como no modelo GBW;

- a condição inicial em rapidez ficou estabelecida em $Y_{0}=4.6$ que corresponde à $x=10^{-2} \mathrm{e}$

- o parâmetro $c$ foi estabelecido em 0.2.

No que segue iremos chamar essa modificação do modelo KKT, descrito na referência [60], de KKTm. Nas figuras 4.8 a 4.11 apresentamos também as previsões do modelo KKTm e verificamos que este implica em um bom acordo com os dados experimentais. 

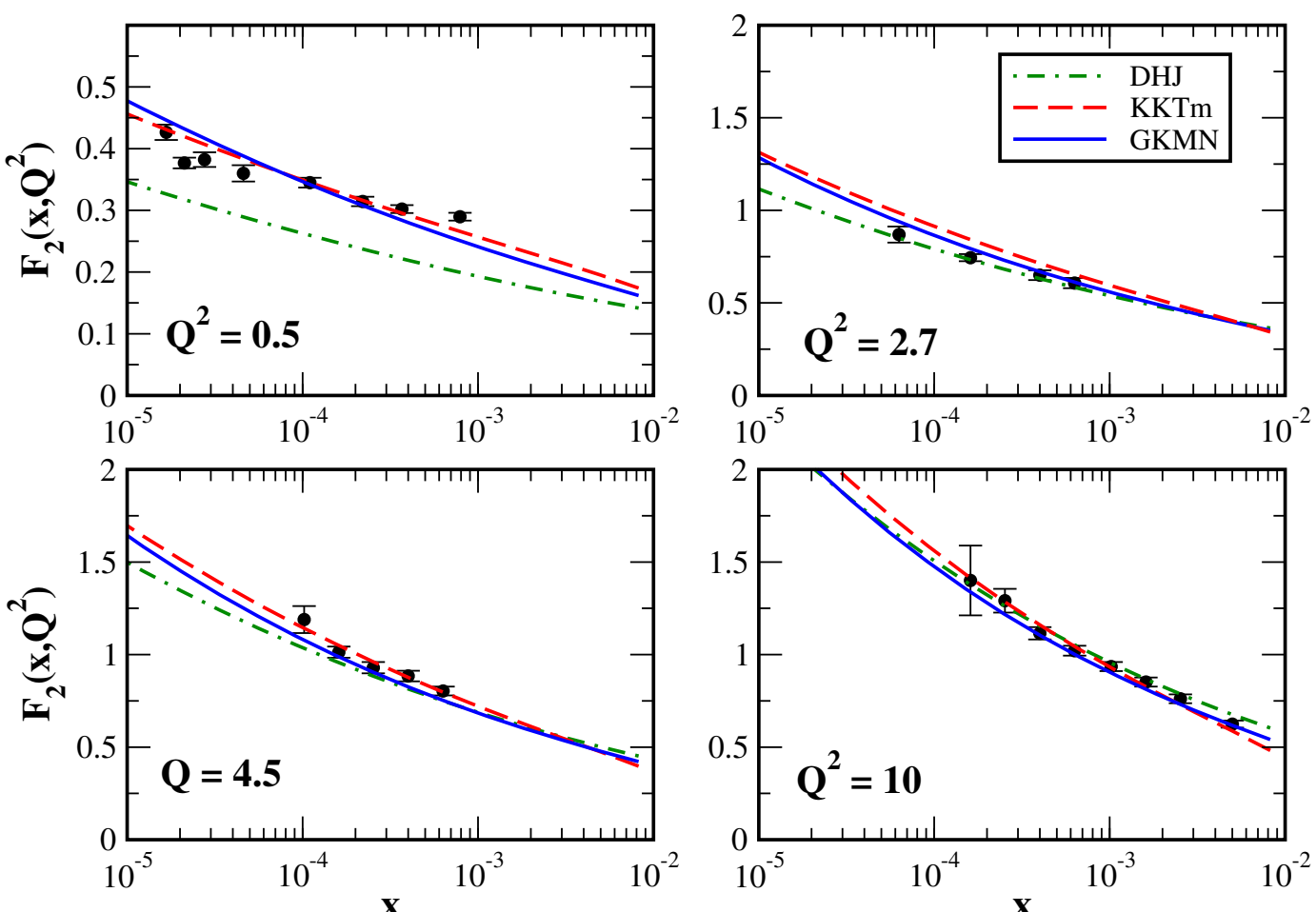

Figura 4.8: Função de estrutura do próton para valores baixos de virtualidades do fóton. Os dados são de ZEUS.

Na referência [8] propusemos modificar o modelo DHJ assumindo que $Q_{T}=Q_{0}=$ $1.0 \mathrm{GeV}^{2}$, ou seja, que a escala típica é independente da energia. No que segue chamaremos essa nova parametrização de GKMN. É importante enfatizar que esta modificação preserva as principais propriedades da dimensão anômala proposta em [56]. Basicamente, ainda temos que a dimensão anômala cresce logaritmicamente com $p_{T}$ de $\gamma=\gamma_{s}$ para seu valor assintótico $\gamma \approx 1$ e decresce com $Y \operatorname{com} \Delta Y \approx 1 / Y$ em valores de rapidez grande. Como mostrado nas figuras 4.8, 4.9, 4.10 e 4.11, com esta modificação nossas previsões (linhas GKMN) concordam com os dados experimentais do HERA.

Nas figuras 4.12 e 4.13 apresentamos a seção de choque de dipolo como uma função da variável de scaling $r Q_{s}$ e em função do tamanho do dipolo $r^{2}$, respectivamente, para diferentes parametrizações. Vemos que os modelos DHJ, KKTm e GKMN pre- 


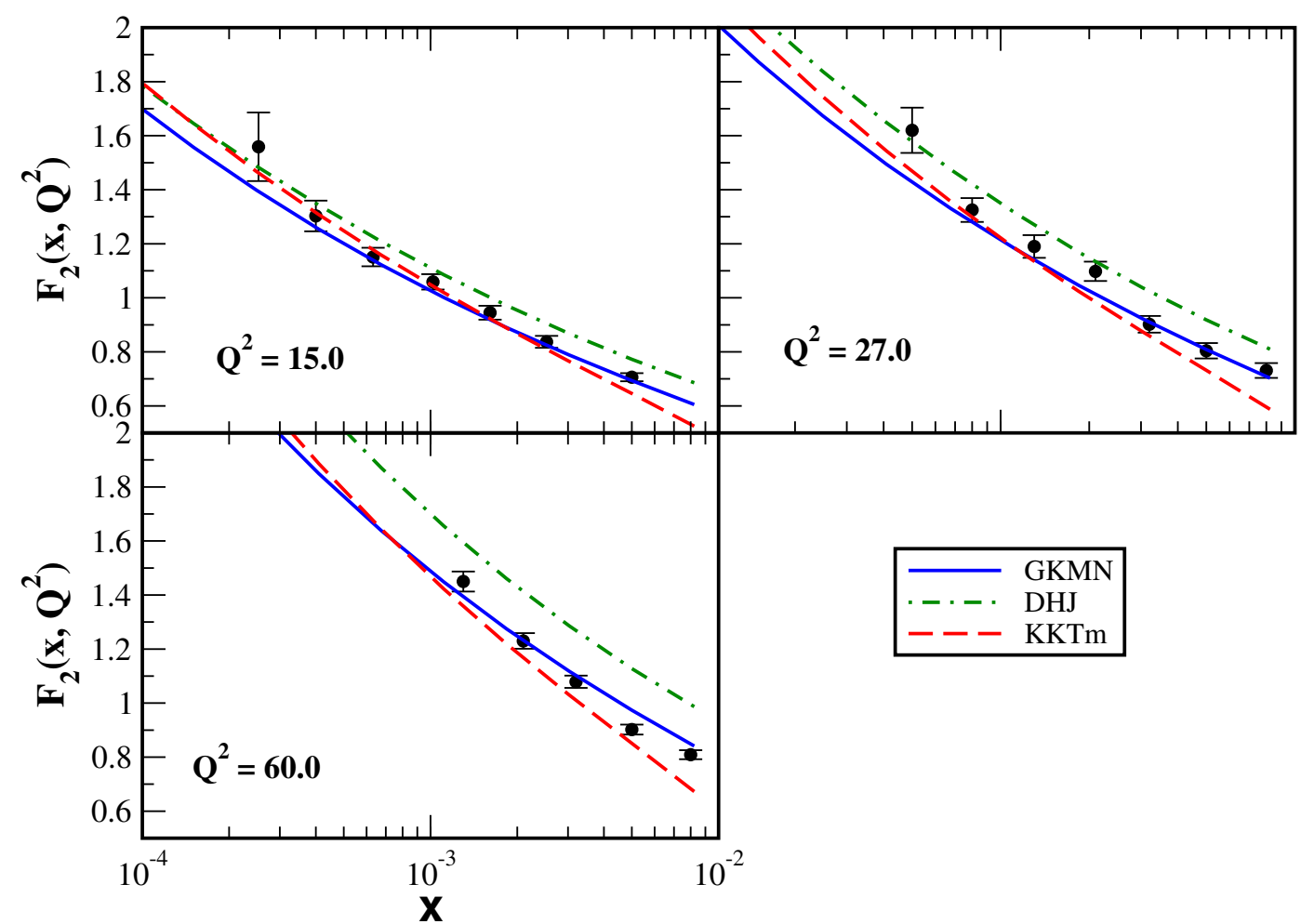

Figura 4.9: Função de estrutura do próton para valores altos de virtualidades do fóton. Os dados são de ZEUS.

vêem comportamentos semelhantes. A diferença entre os modelos pode ser explicitada estudando o comportamento $Q^{2}$ da dimensão anômala efetiva, definida por [60]

$$
\gamma_{e f f}=\frac{d \ln \mathcal{N}\left(r Q_{s}, Y\right)}{d \ln \left(r^{2} Q_{s}^{2} / 4\right)}
$$

Análises parecidas foram feitas na referência [60]. Nas figuras 4.14 e 4.15 é mostrado $\gamma_{\text {eff }}$ como uma função da virtualidade $Q^{2}$ e da variável de scaling, respectivamente, usando um tamanho médio de dipolo de $r=2 / Q$. Enquanto o modelo GBW apresenta uma rápida convergência para a dimensão anômala em grande $Q^{2}$, a parametrização IIM tem um crescimento moderado convergindo para $\gamma \approx 0.85$ em grande $Q^{2}$. As parametrizações KKTm e IIM são semelhantes em grande $Q^{2}$, mas diferem em pequenas virtualidades, com a KKTm prevendo um valor menor. Por outro lado, as previsões 

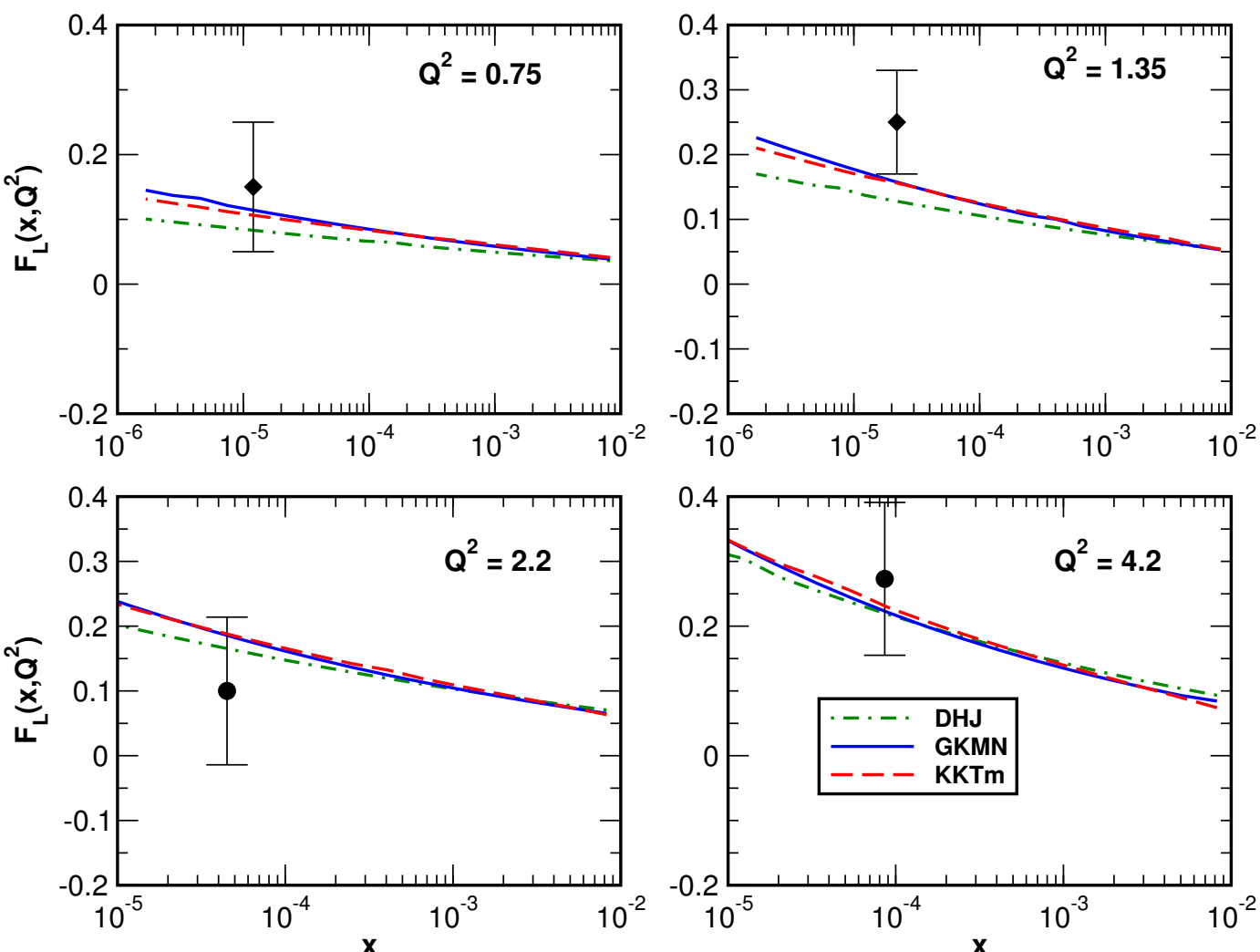

Figura 4.10: Função de estrutura longitudinal do próton para valores baixos de virtualidades do fóton. Os dados são de ZEUS.

das parametrizações DHJ e GKMN são parecidas em pequeno $Q^{2}$ e diferem em grandes virtualidades. Em particular, temos uma diferença entre esses modelos no regime de virtualidades intermediárias que pode explicar porque o modelo DHJ não descreve a evolução em $Q^{2}$ da função de estrutura $F_{2}$.

\subsection{Produção de hadrons carregados}

A questão que permanece em aberto é se as parametrizações KKTm e GKMN, as quais descrevem os dados de HERA também descrevem aos dados de RHIC. No que segue calculamos a seção de choque inclusiva de produção de hádron carregados em colisões $d A u$ para diferentes valores de rapidez usando as parametrizações para a amplitude 

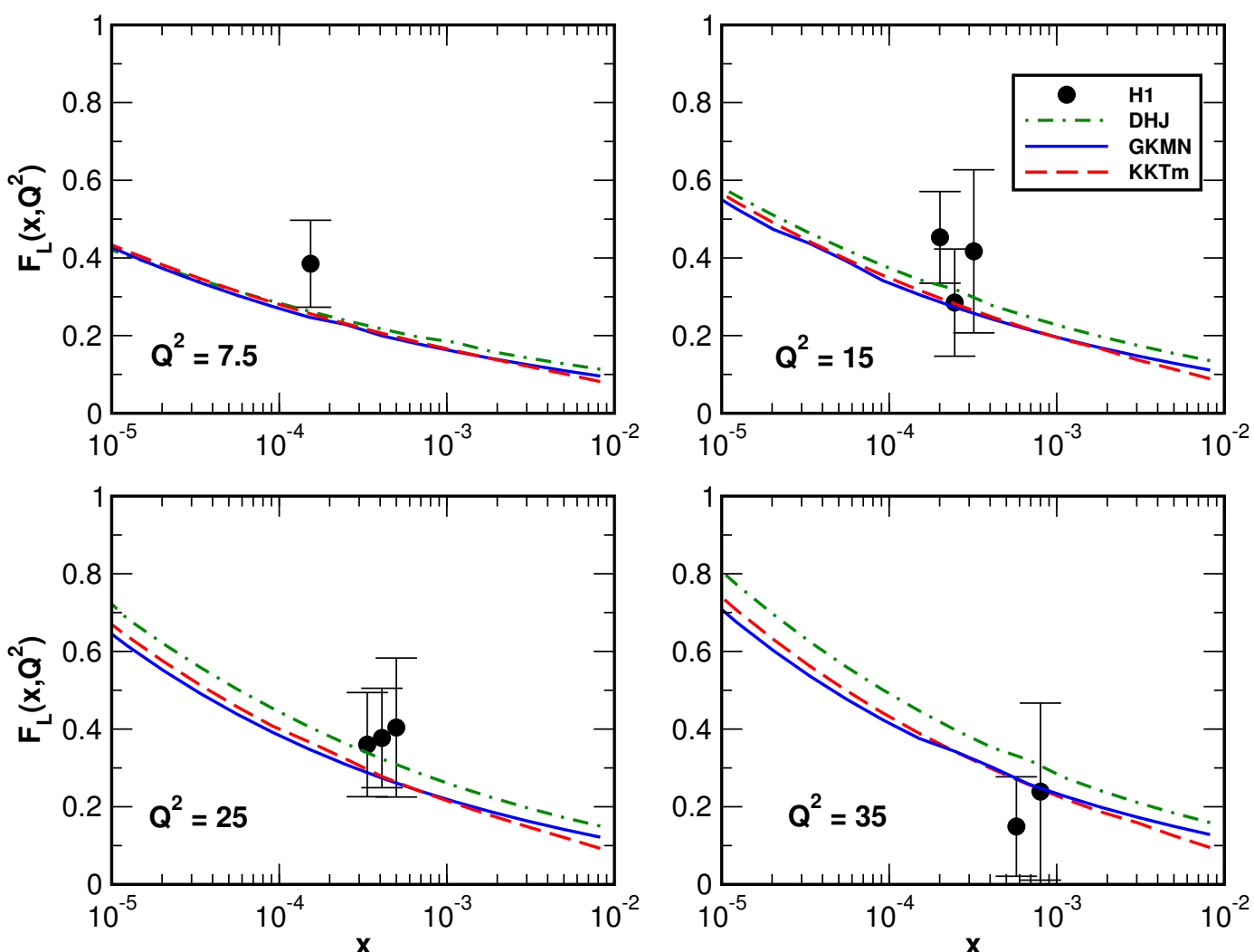

Figura 4.11: Função de estrutura longitudinal do próton para valores altos de virtualidades do fóton. Os dados são de ZEUS.

de espalhamento, $\mathcal{N}$.

A produção inclusiva de hádrons em processos hádron-hádron é descrita no formalismo do CGC como [56]:

$$
\begin{aligned}
x_{F} \frac{d \sigma^{p p(A) \rightarrow h X}}{d x_{F} d^{2} p_{t} d^{2} \boldsymbol{b}}= & \frac{1}{(2 \pi)^{2}} \int_{x_{F}}^{1} d x_{p} \frac{x_{p}}{x_{F}}\left[f_{q / p}\left(x_{p}, Q_{f}^{2}\right) \mathcal{N}_{\mathcal{F}}\left(\frac{x_{p}}{x_{F}} p_{t}, \boldsymbol{b}\right) D_{h / q}\left(\frac{x_{F}}{x_{p}}, Q_{f}^{2}\right)+\right. \\
& \left.f_{g / p}\left(x_{p}, Q_{f}^{2}\right) \mathcal{N}_{\mathcal{A}}\left(\frac{x_{p}}{x_{F}} p_{t}, \boldsymbol{b}\right) D_{h / g}\left(\frac{x_{F}}{x_{p}}, Q_{f}^{2}\right)\right],
\end{aligned}
$$

onde $p_{t}$ e $x_{F}$ são o momento transversal e o $x$ de Feynman do hádron produzido, respectivamente. A variável $x_{p}$ denota a fração de momento de um párton do projétil e b o parâmetro de impacto. Além disso, $f\left(x_{p}, Q_{f}^{2}\right)$ é a função de distribuição de 


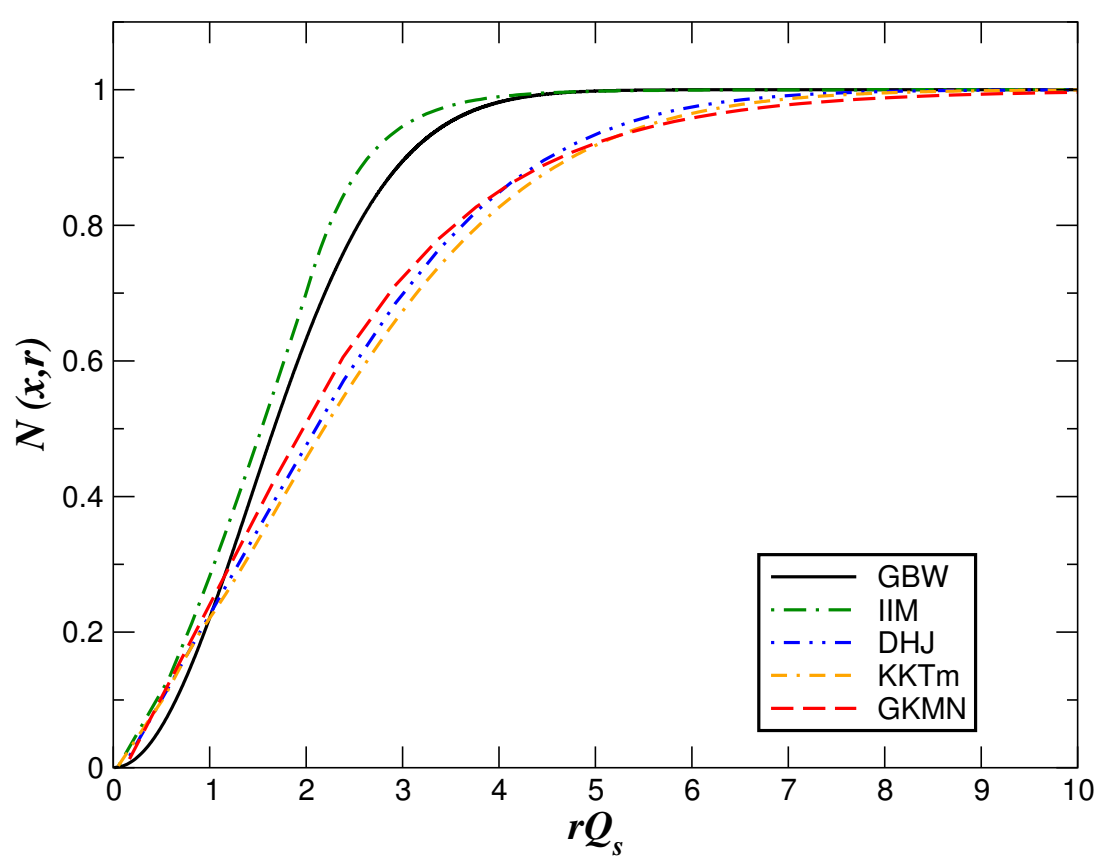

Figura 4.12: Amplitude de espalhamento do dipolo em função da variável de scaling $r Q_{s}$.

pártons do projétil e $D\left(z, Q_{f}^{2}\right)$ é a função de fragmentação de partons em hadrons. Estas quantitades evoluem de acordo com as equações de evolução DGLAP [38] e respeitam a regra de soma dos momentos. Na Eq. $(4,2.5), \mathcal{N}_{\mathcal{F}}(\boldsymbol{k}, \boldsymbol{b})$ e $\mathcal{N}_{\mathcal{A}}(\boldsymbol{k}, \boldsymbol{b})$ são as amplitudes de espalhamento de dipolos (no espaço dos momentos) nas representações fundamental e adjunta respectivamente. Vemos que $\mathcal{N}_{\mathcal{A}, \mathcal{F}}(\boldsymbol{k}, \boldsymbol{b})$ e $\mathcal{N}_{\mathcal{A}, \mathcal{F}}(\boldsymbol{r}, \boldsymbol{b})$ estão diretamente relacionadas por uma transformada de Fourier.

Em nosso estudo usamos a distribuição de quarks e gluons CTEQ5L [18] e as funções de fragmentação LO KKP [61]. Apresentamos nossos resultados na figura 4.16 e comparamos com os dados de BRAHMS [62]. As predições KKTm e GKMN são as curvas tracejada-longa e sólida, respectivamente. Como na referência [56] precisamos de um fator $K$ em nosso cálculo, visto que este foi calculado em ordem dominante em $\alpha_{s}$. Embora a normalização deva ser modificada por correções de mais alta ordem, esperamos que a forma da distribuição de momento não seja alterada. Nossos valores de $K$ foram determinados de tal forma a reproduzir os dados de $p_{T}=1.0 \mathrm{GeV}$ e eles 


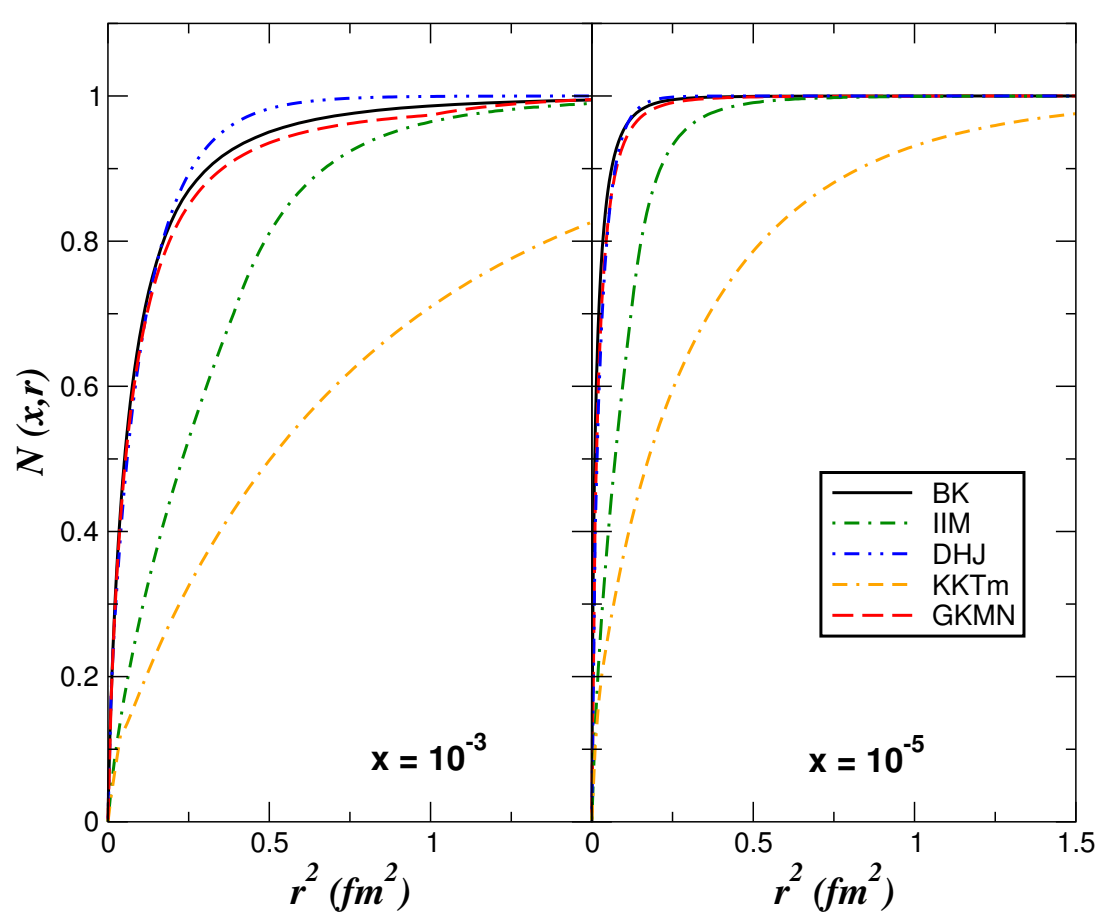

Figura 4.13: Amplitude de espalhamento do dipolo em função do tamanho do dipolo $r^{2}$.

dependem da parametrização adotada. Para KKTm encontramos um valor maior de $K$ do que para GKMN. Além disso, enquanto a parametrização KKTm falha para descrever o conjunto completo dos dados, a GKMN é capaz de reproduzir os dados com boa precisão sobretudo para valores menores de $p_{T}$. Consequentemente, o modelo GKMN é capaz de descrever os dados de ep no HERA e $d A u$ no RHIC em termos de uma única parametrização para a amplitude de espalhamento de dipolo, demonstrando a propriedade de universalidade predita pelo física do CGC. 


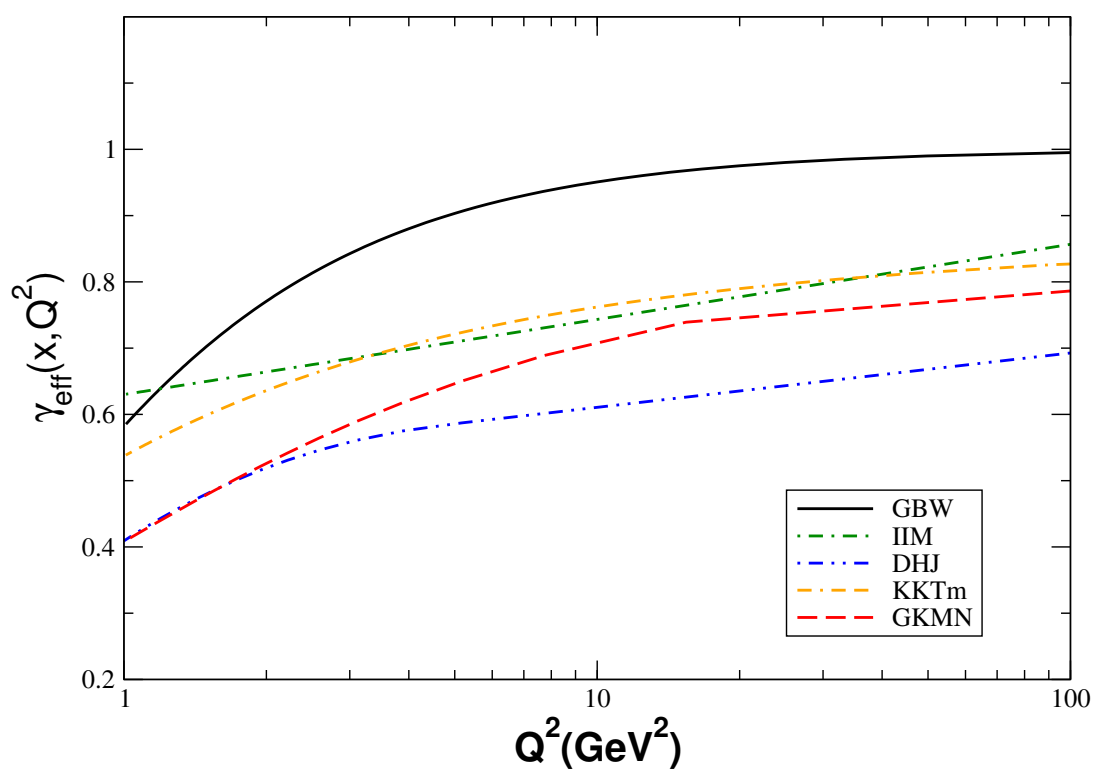

Figura 4.14: $O$ comportamento em $Q^{2}$ da dimensão anômala efetiva.

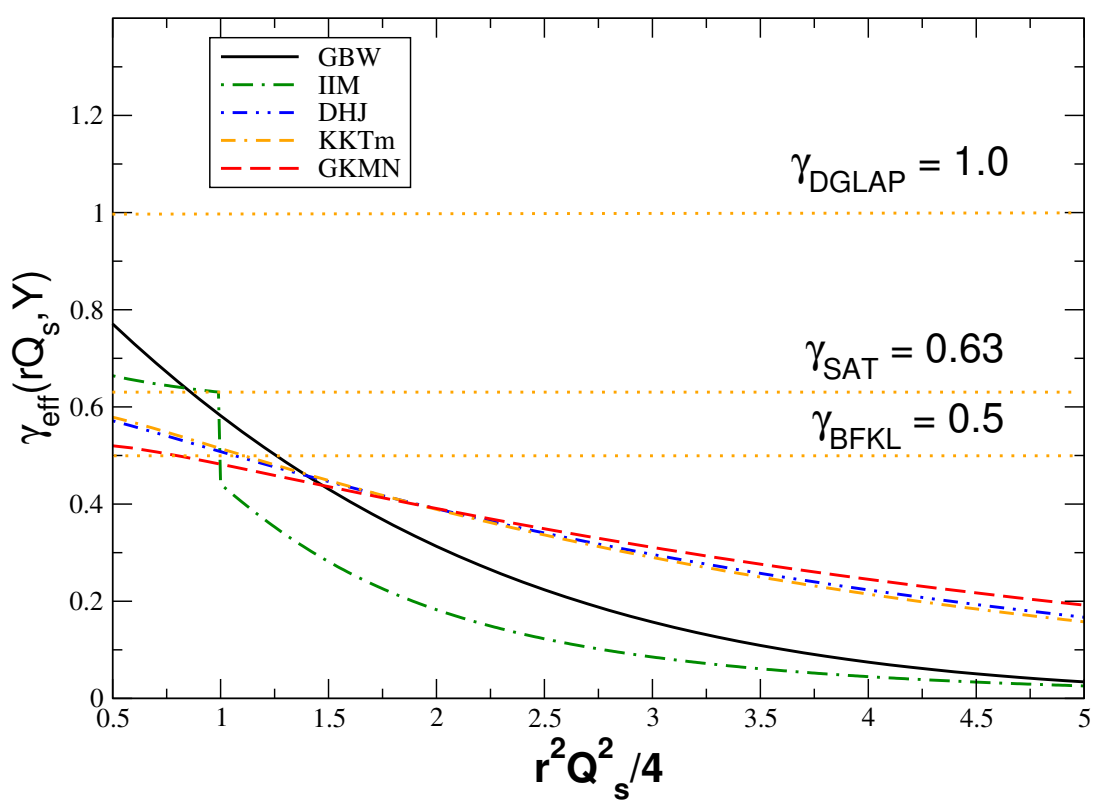

Figura 4.15: $O$ comportamento em $r Q_{s}$ da dimensão anômala efetiva. 

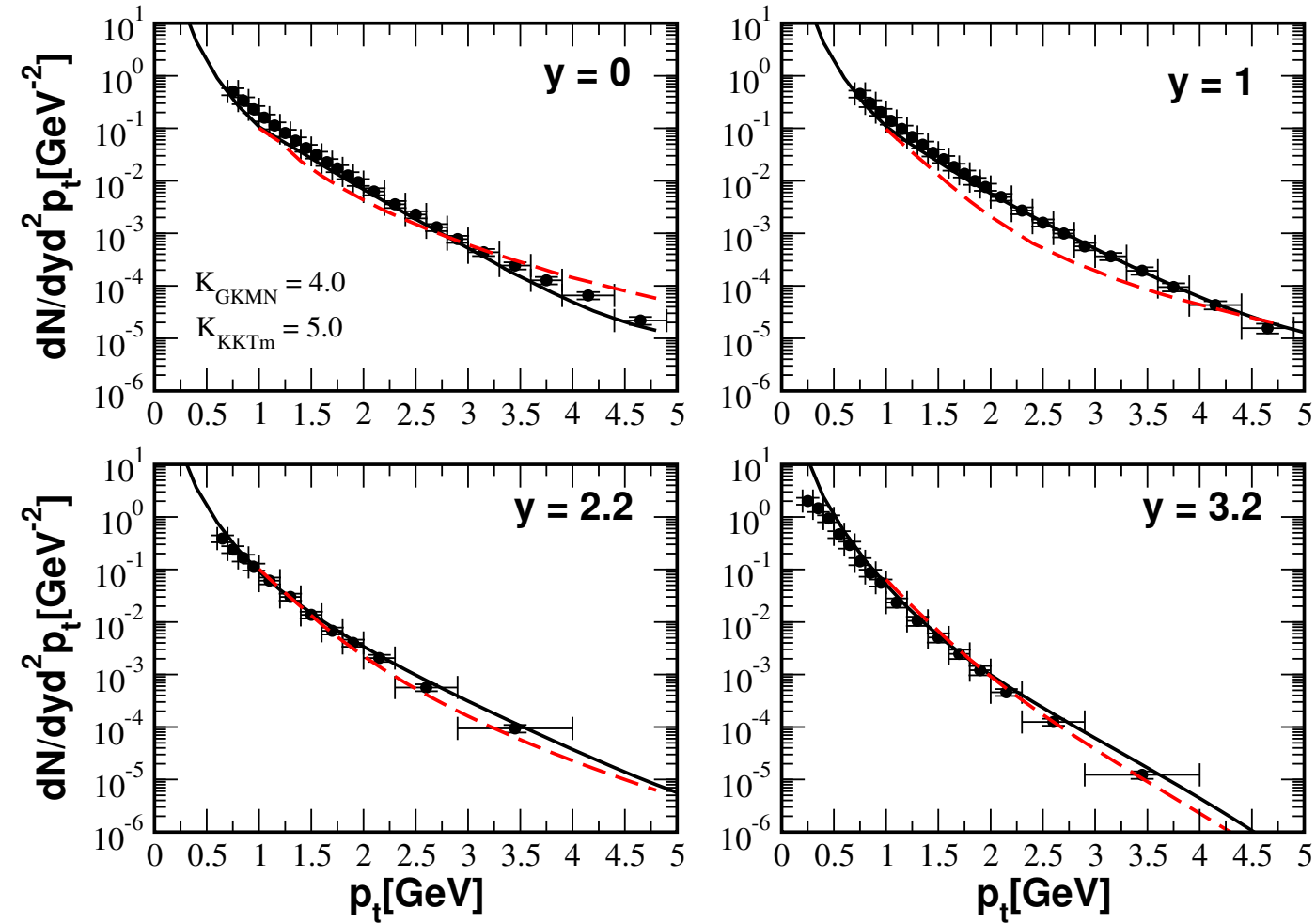

Figura 4.16: Comparação entre teoria e dados de BRAHMS para colisões minimum-bias $d A u$ na energia do RHIC. 


\section{Capítulo 5}

\section{Física de saturação em processos $e A$}

Como discutido nos capítulos anteriores, a escala de saturação cresce com o número atômico. Portanto, processos nos quais um núcleo esteja presente são ideais para testar a física de saturação. Nos últimos anos diversos pesquisadores têm defendido a construção de um colisionador elétron-núcleo $(e A)$ a fim de testar a QCD no regime de altas energias em um meio nuclear. Em particular, a proposição da construção de um colisionador $e A$ no RHIC foi apresentada na referência [63].

Aqui estudamos o comportamento da função de estrutura total, longitudinal e para a contribuição do charme, na região cinemática que será acessível em colisões elétroníon no RHIC. Fazendo a generalização para alvos nucleares do modelo de saturação proposto por Iancu, Itakura e Munier (IIM). Além disso, estimamos os slopes logarítmicos da função de estrutura total e longitudinal em diferentes valores do número atômico. Esperamos que nossos resultados contribuam para o planejamento do futuro experimento $e A$.

Neste capítulo apresentamos os resultados das referências [10, 9]. 


\subsection{Espalhamento elétron-núcleo}

\subsubsection{A função peso nuclear inclusiva}

Generalizamos o modelo IIM para colisões nucleares, assumindo as seguintes transformações básicas:

$$
\begin{gathered}
\sigma_{0} \rightarrow \sigma_{0}^{A}=A^{\frac{2}{3}} \times \sigma_{0} \\
\mathrm{e} \\
Q_{s}^{2}(x) \rightarrow Q_{s, A}^{2}=A^{\frac{1}{3}} \times Q_{s}^{2}(x) .
\end{gathered}
$$

Além disso, para estimar a contribuição da física de saturação apresentamos uma comparação entre o modelo IIM completo e as predições da física linear, obtida extrapolando a expressão de $\mathcal{N}(x, \boldsymbol{r})$, válida para $\boldsymbol{r} Q_{s}(x) \leq 2$, para todo o alcance cinemático. Antes de apresentarmos nossos resultados para a função de estrutura total, longitudinal e do charme, podemos investigar a importância do tamanho do dipolo dominante para cada seção de choque. Definimos a função peso (função de "overlap") fóton-núcleo por:

$$
H_{i}\left(\boldsymbol{r}, x, Q^{2}\right)=2 \pi r \int d z\left|\Psi_{i}\left(z, \boldsymbol{r}, m_{f}, Q^{2}\right)\right|^{2} \sigma_{d i p}(x, \boldsymbol{r}, A)
$$

onde $i=T, L$ caracteriza o fóton transverval e longitudinal, respectivamente. Em particular também calculamos a função peso associada à função de estrutura total $H_{2}\left(\boldsymbol{r}, x, Q^{2}\right) \equiv H_{T}\left(\boldsymbol{r}, x, Q^{2}\right)+H_{L}\left(\boldsymbol{r}, x, Q^{2}\right)$, e a função peso associada à função de estrutura do charme $H_{c}\left(\boldsymbol{r}, x, Q^{2}\right)$, que é calculada usando-se $m_{f}=m_{c}=1.5 \mathrm{GeV}$. Nas figuras 5.1, 5.2 e 5.3 mostramos as diferentes funções peso (normalizadas por A) como uma função do tamanho do dipolo para diferentes valores de $x, A$ e $Q^{2}$. O primeiro aspecto que deve ser enfatizado é que, embora as funções peso estejam normalizadas por $A$, elas são fortemente dependentes de $A$. Este comportamento é esperado quando consideramos a predição completa do modelo IIM. Além disso nossos 
resultados demonstram que esta dependência está também presente quando calculamos as funções peso usando a aproximação linear. Isto está associado a dependência de $\left[Q_{s, A}^{2}(x)\right]^{\gamma_{e f f}}$ com a amplitude de espalhamento de dipolo, onde no modelo IIM $\gamma_{\text {eff }}=$ $\gamma_{s}+\frac{\ln \left(2 / \boldsymbol{r} Q_{s}\right)}{\kappa \lambda Y}$. Para $\gamma_{e f f}=1$ teremos a seguinte dependência para $\mathcal{N}$ em $A: A^{\frac{1}{3}}$, que combinada com a dependência $A^{\frac{2}{3}}$ de $\sigma_{0}$ implica numa dependência linear em $A$ para a seção de choque de dipolo no regime linear. Se normalizada por $A$, obteremos uma função peso independente de $A$ no regime linear. Como $\gamma_{e f f}<1$, no modelo IIM, resulta que a função peso (e os observáveis correspondentes) têm uma dependência $A^{\frac{\gamma_{e f f}-1}{3}}$, ou seja, ela decresce com o aumento do número atômico. Este comportamento é observado nas figuras.

Na figura 5.1, estimamos a função peso para $x\left(=10^{-5}\right)$ e $Q^{2}\left(=1 \mathrm{GeV}^{2}\right)$ fixos e para dois valores de número atômico. Podemos ver que a função peso do charme tem um pico em $\boldsymbol{r} \approx 0.1 \mathrm{fm}$, que está de acordo com a expectativa teórica de que o par $c \bar{c}$ tenha um tamanho transversal típico $\approx 1 / m_{c}$. Portanto a contribuição para a seção de choque vem de pequenos dipolos, ou seja, da região onde os efeitos de saturação são pequenos (regime linear). Esta expectativa é verificada no comportamento da função peso $H_{c}$, que apresenta um comportamento idêntico quando comparamos as predições linear e completa (linear + não-linear) para um $A$ fixo. Assim, podemos esperar que modificações na função de estrutura do charme, devido aos efeitos de saturação sejam pequenas. Por outro lado, para a produção de quarks leves uma distribuição larga em $\boldsymbol{r}$ é obtida, centrada em grandes separações do par, implicando que os efeitos de saturação contribuem significativamente neste caso. Este comportamento também é verificado para as funções peso $H_{2}$ e $H_{L}$ apresentadas na figura 5.1. Neste caso vemos que os efeitos de saturação suprimem a contribuição para grandes dipolos, como esperado teoricamente. Além disso esses efeitos tornam-se mais importantes para pequenos valores de $x$ e grandes valores de $A$. Podemos observar que a área sob a curva é reduzida significativamente pelos efeitos de saturação, o que implica que os observáveis associados serão fortemente modificados por esses efeitos.

$\mathrm{Na}$ figura 5.2, mostramos o comportamento de $H_{L}$ e $H_{2}$ para $Q^{2}=10 \mathrm{GeV}^{2}$. 

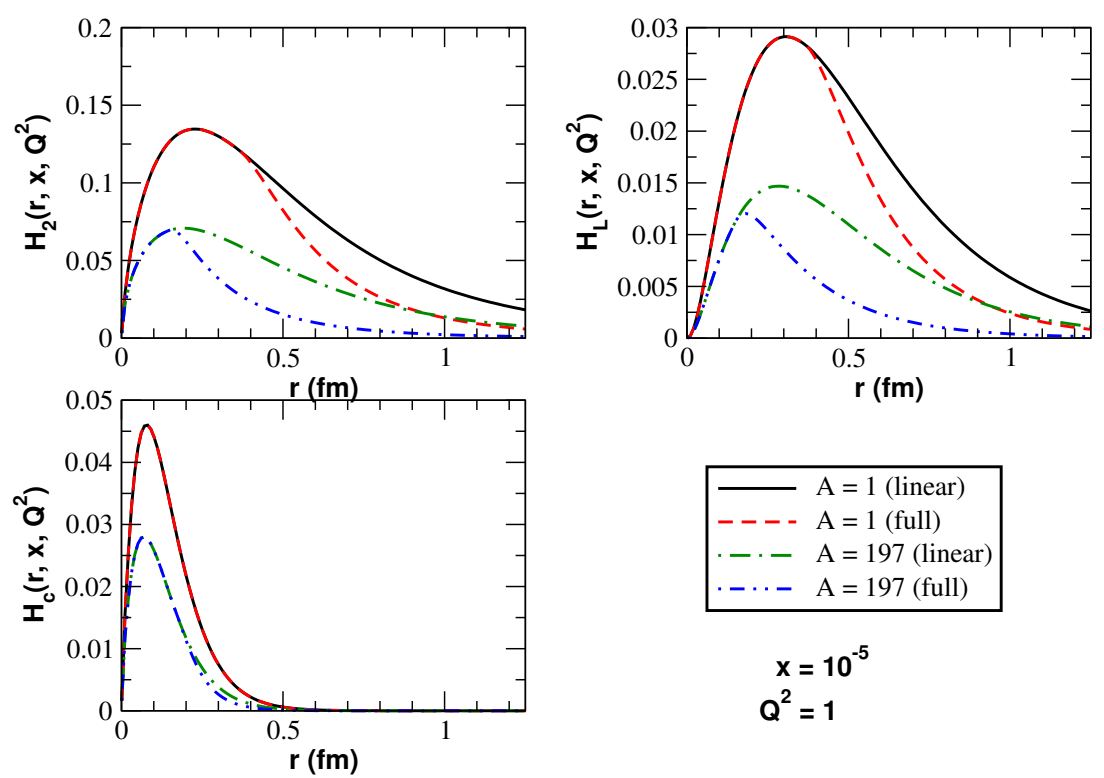

Figura 5.1: A dependência em $r$ da função peso fóton-núcleo para diferentes valores de número atômico $\left(x=10^{-5}\right.$ e $\left.Q^{2}=\mathbf{1} \mathbf{G e V}^{2}\right)$.

Neste caso observamos um pico para a distribuição de pequenos valores de tamanho de dipolo, sendo que a contribuição para grandes valores de tamanho de dipolo é reduzida. Isto implica que para $A=1$ as predições completa e linear são idênticas. Para núcleos maiores $(A=197)$ os efeitos de saturação ainda contribuem e levam a uma redução na área sob a curva e a uma modificação associada a esse observável. A função peso do charme (não mostrada na figura 5.2) tem um comportamento idêntico para as predições linear e completa e para dois valores de número atômico. Podemos concluir que os efeitos de saturação são fortemente reduzidos para grandes valores de $Q^{2}$.

Na figura 5.3 mostramos as funções peso para dois valores de $x$ e $Q^{2}$. Nesta figura mostramos apenas a predição completa. Como discutido anteriormente:

1. Aumentando $Q^{2}$ a distribuição é centrada para pequenos valores de tamanho de dipolo; e

2. A área sob a curva é reduzida aumentando-se o número atômico $A$.

O principal aspecto dessa figura é que ela permite analisar a dependência em $x$ da função peso. Observamos que, diminuindo $x$, a função peso cresce, com o crescimento 

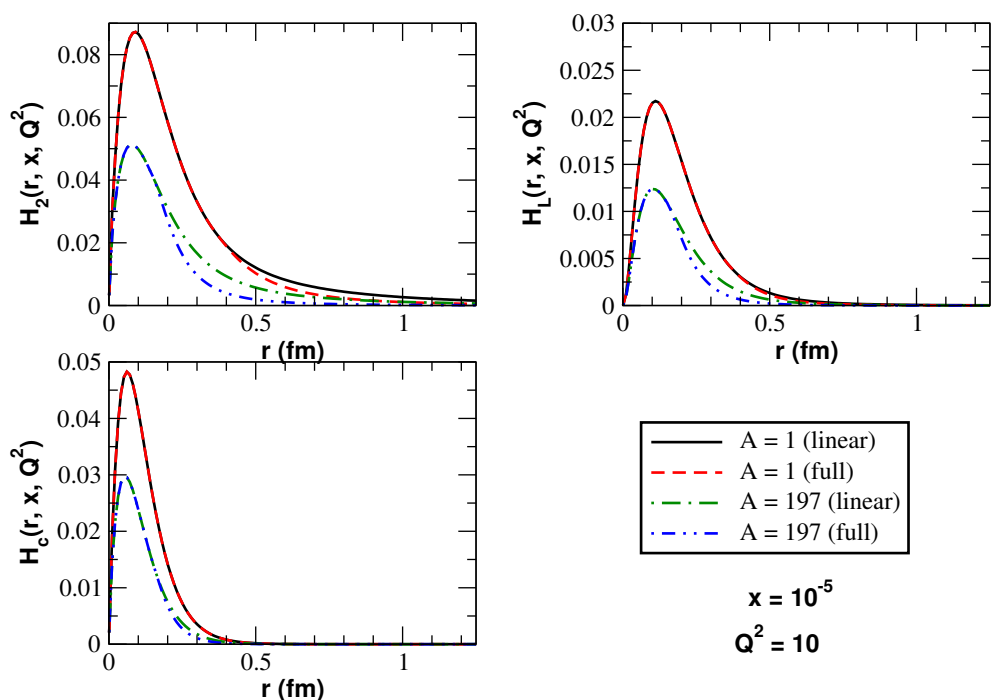

Figura 5.2: $A$ dependência em $r$ da função peso fóton-núcleo para diferentes valores de número atômico $\left(x=10^{-5}\right.$ e $\left.Q^{2}=10 \mathbf{G e V}^{2}\right)$.

sendo menor para grandes núcleos. Consequentemente esperamos que os observáveis associados aumentem em pequeno $x$, com uma inclinação menor para $A=197$.

\subsubsection{A função peso nuclear difrativa}

Antes de apresentar nossos resultados para $\frac{\sigma_{d i f f}}{\sigma_{t o t}}$ e para a função de estrutura difrativa, vamos investigar o tamanho de dipolo dominante na seção de choque difrativa, através da análise da função peso difrativa fóton-núcleo definida por

$$
H_{D}\left(\boldsymbol{r}, x, Q^{2}\right)=2 \pi r \sum_{i=T, L} \int d \alpha\left|\Psi_{i}\left(\alpha, \boldsymbol{r}, Q^{2}\right)\right|^{2} \sigma_{d i p}^{2}(x, \boldsymbol{r}, A)
$$

Nas figuras 5.4 e 5.5 apresentamos a dependência em $\boldsymbol{r}$ da função peso difrativa fótonnúcleo (normalizada por $A^{2}$ ) em diferentes valores de número atômico e $Q^{2}=1 \mathrm{GeV}^{2}$. Uma análise semelhante pode ser feita para outros valores de $Q^{2}$. A principal diferença é que para grandes valores de $Q^{2}$, a função peso tem um pico em pequenos valores de separação do par. Por comparação as previsões obtidas com uma generalização do modelo de Golec-Biernat Wüsthoff (GBW) [26] para núcleos também é apresen- 

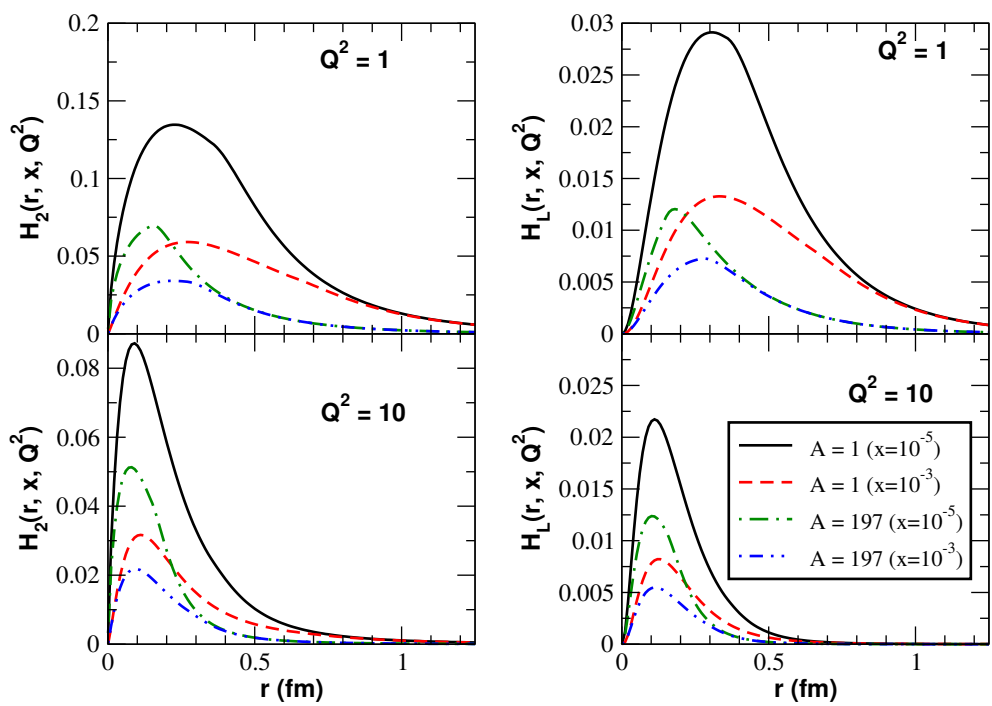

Figura 5.3: A dependência em $r$ da função peso fóton-núcleo para diferentes valores de número atômico, $x$ e $Q^{2}$.

tada. Quando os efeitos de saturação são incluídos na função peso IIM e GBW, elas apresentam um comportamento semelhante, reduzindo fortemente a contribuição para grandes dipolos. Em grande valores de $A$ apenas pequenas separações do par contribuem para a seção de choque difrativa. Entretanto, no caso linear, esses dois modelos apresentam um comportamento muito distinto, o qual está diretamente associado com as diferentes previsões para o regime linear. Enquanto o modelo GBW assume que $\sigma_{d i p} \propto \sigma_{0} \boldsymbol{r}^{2} Q_{s}^{2}$ no regime linear, o modelo IIM assume que $\sigma_{d i p} \propto \sigma_{0}\left[\boldsymbol{r}^{2} Q_{s}^{2}\right]^{\gamma_{\text {eff }}}$, onde $\gamma_{e f f}=\gamma_{s}+\ln \left(2 / \boldsymbol{r} Q_{s}\right) / \kappa \lambda Y$ é muito menor do que um. Primeiramente isso leva a uma diferente dependência em $A$, pois no modelo GBW o produto $\left[\sigma_{0} Q_{s}^{2}\right]^{2}$ é proporcional a $A^{2}$, o qual cancela com o termo da normalização. No modelo IIM temos $\left[\sigma_{0} Q_{s}^{2 \gamma_{e f f}}\right]^{2}$, o qual leva em uma dependência $A^{\frac{4+2 \gamma_{e f f}}{3}}$. Quando combinado com o fator $A^{2}$, o qual vem da normalização, esperamos uma dependência $A^{\frac{2\left(\gamma_{e f f}-1\right)}{3}}$ para a função peso de IIM. Como $\gamma_{e f f}<1$, a função peso decresce para grandes $A$ também no regime linear. Este comportamento é visto nas figuras 5.4 e 5.5. Ao contrário do modelo GBW que prevê um comportamento $\boldsymbol{r}^{2}$ para seção de choque de dipolo no regime linear, o modelo IIM leva a uma dependência $\boldsymbol{r}^{2 \gamma_{\text {eff }}}$. Esta previsão diferente para a dependência em 

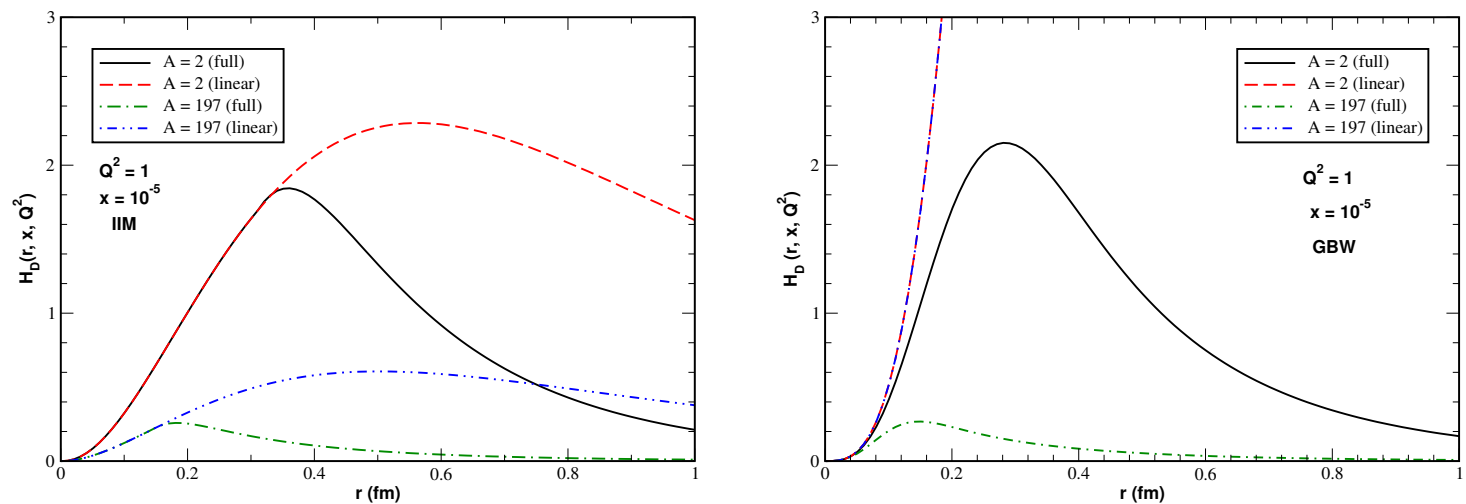

Figura 5.4: A dependência em $r$ da função peso difrativa fóton-núcleo (normalizada por $A^{2}$ ) em diferentes valores do número atômico e distintos modelos de saturação: IIM (painel à esquerda) e GBW (painel à direita).

$\boldsymbol{r}$, quando combinada com a dependência da separação do par nas funções de onda, leva a uma grande modificação, da contribuição de grandes dipolos, como observado na figura 5.4 e também na figura 5.5. É importante ressaltar que esta contribuição domina a seção de choque, isto é, se desprezarmos os efeitos de saturação, a seção de choque difrativa será dominada pela física soft.
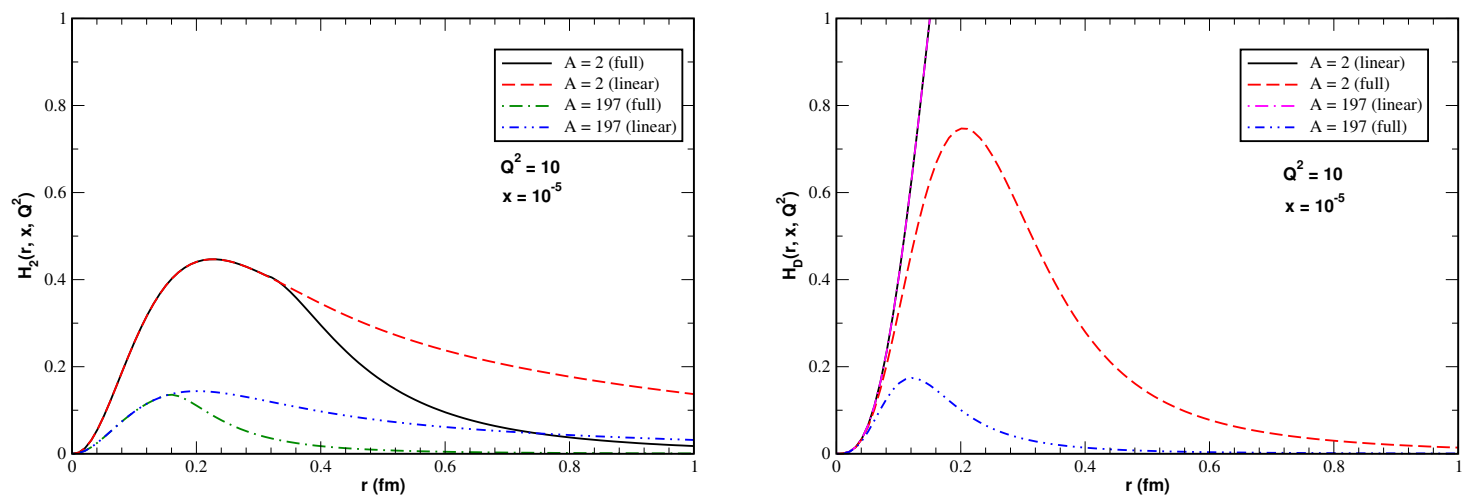

Figura 5.5: A dependência em $r$ da função peso difrativa fóton-núcleo (normalizada por $A^{2}$ ) em diferentes valores do número atômico e distintos modelos de saturação: IIM (painel à esquerda) e GBW (painel à direita). 


\subsubsection{A função de estrutura nuclear inclusiva}

As expectativas dos observáveis associados à função peso inclusiva são mostradas na figura 5.6, onde mostramos a dependência em $x$ das funções de estrutura total, longitudinal e de charme. Vemos que as predições completa e linear para o charme são idênticas, apenas tendo uma dependência em $A$ característica do modelo IIM. Por outro lado, o comportamento de $F_{2}^{A}$ e $F_{L}^{A}$ é fortemente modificado pela física de saturação, com o efeito decrescendo para grandes valores de $Q^{2}$. A fim de obter uma estimativa mais precisa da modificação desses observáveis com respeito a saturação, apresentamos nas figuras 5.7 e 5.8 as razões $R_{F_{2}}$ e $R_{F_{L}}$ entre as predições completa e linear para $F_{2}$ e $F_{L}$, respectivamente. Consideramos três valores típicos de número atômico. Como esperado a contribuição da física de saturação aumenta para grandes núcleos e pequenos valores de $x$. Em particular os valores de $x$ ao redor de $10^{-5}$ predizem uma redução por volta de $50 \%$ nas funções de estrutura total e longitudinal.

$\mathrm{Na}$ figura 5.9, mostramos a razão entre as funções de estrutura nuclear como uma função de $x$ para diferentes valores de $A_{2}$ e fixos $Q^{2}=10 \mathrm{GeV}^{2}$ e $A_{1}=1$.

Outros observáveis de interesse para estudar a física do CGC são as derivadas logarítmicas de $F_{2}$ e $F_{L}$ com respeito a $x$ e $Q^{2}$. Isto é motivado principalmente pela relação estreita entre a distribuição de glúons e a violação de scaling da função de estrutura total em ordem dominante no formalismo de DGLAP [64]. No formalismo de dipolo a violação de scaling está diretamente relacionada à seção de choque de dipolo [65]:

$$
\frac{d F_{2}\left(x, Q^{2}\right)}{d \log Q^{2}} \approx Q^{2} \times \sigma_{d i p}\left(x, \boldsymbol{r}^{2}=\frac{4}{Q^{2}}\right)
$$

Portanto esse observável deve ser útil para definir o limite entre os regimes linear e de saturação [66]. Como esse observável depende fortemente da seção de choque de dipolo e apresenta um comportamento distinto para $Q^{2}>Q_{s}^{2}$ e $Q^{2}<Q_{s}^{2}$, sua análise experimental permitiria testar a dependência da escala de saturação com $x \mathrm{e}$ A. Por outro lado, a derivada logarítmica de $F_{2}$ com respeito a $x$ está diretamente 


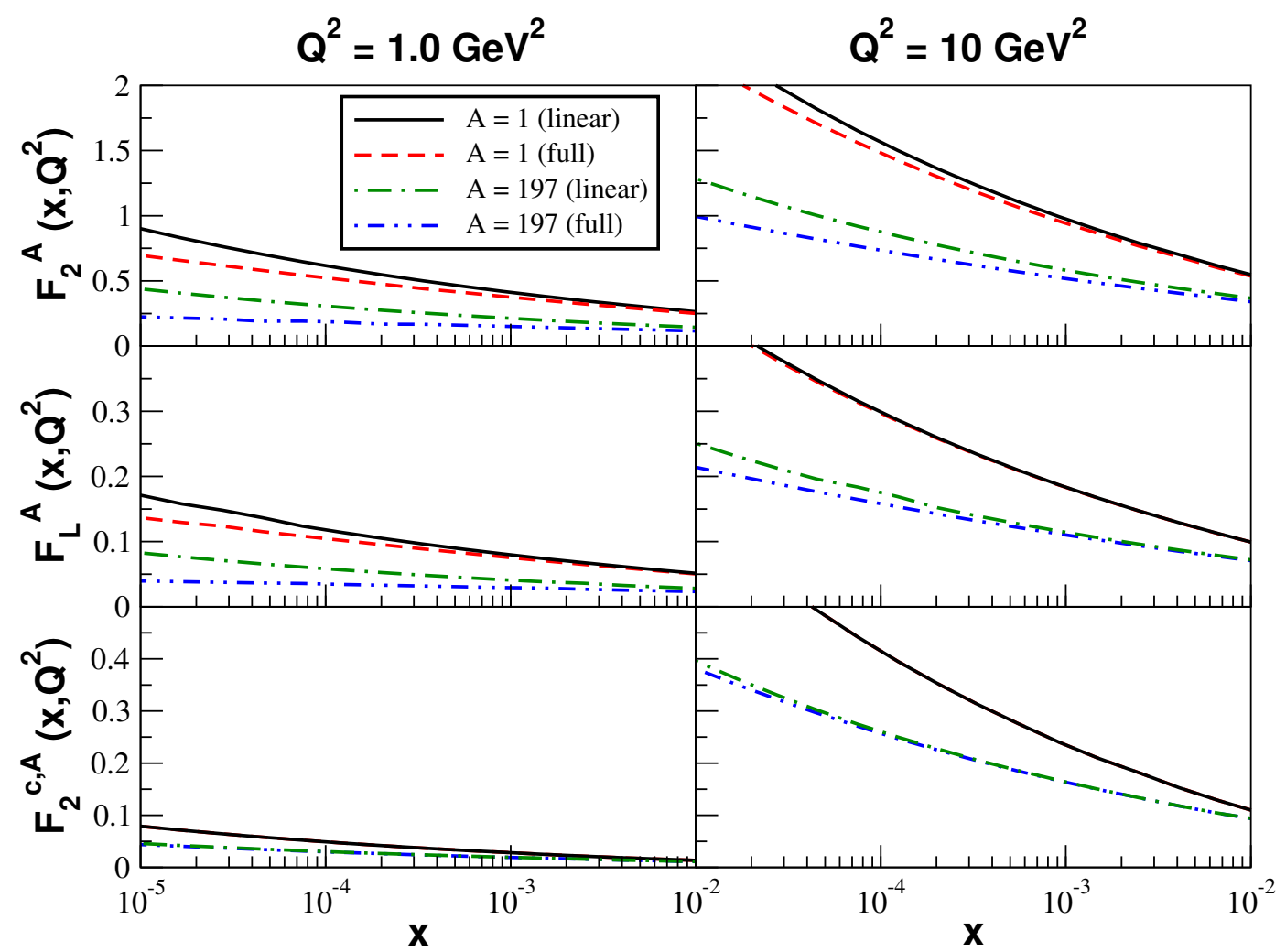

Figura 5.6: Funções de estrutura nuclear como uma função de $x$ para diferentes valores de $A$ e $Q^{2}$.

relacionada com a potência de crescimento da função de estrutura em pequeno $x$. Se parametrizarmos a função de estrutura total usando $F_{2}^{A}\left(x, Q^{2}\right)=x^{-\lambda\left(x, Q^{2}, A\right)}$ obtemos que

$$
\frac{d \log F_{2}^{A}\left(x, Q^{2}\right)}{d \log 1 / x}=\lambda\left(x, Q^{2}, A\right)
$$

Na figura 5.10(a) mostramos a dependência em $Q^{2}$ do expoente efetivo para $x=10^{-3}$ e diferentes valores de número atômico. Por comparação a predição do modelo GBW generalizada para alvos nucleares é também apresentada. Para pequenos valores de $Q^{2}$ ambos os modelos predizem uma dependência semelhante para $\lambda$. A principal diferença ocorre na região de grandes valores de $Q^{2}$ onde a predição desses modelos pode não ser válida. Além disso, uma dependência em $A$ é observada para $\lambda$, sendo $\lambda$ menor para 

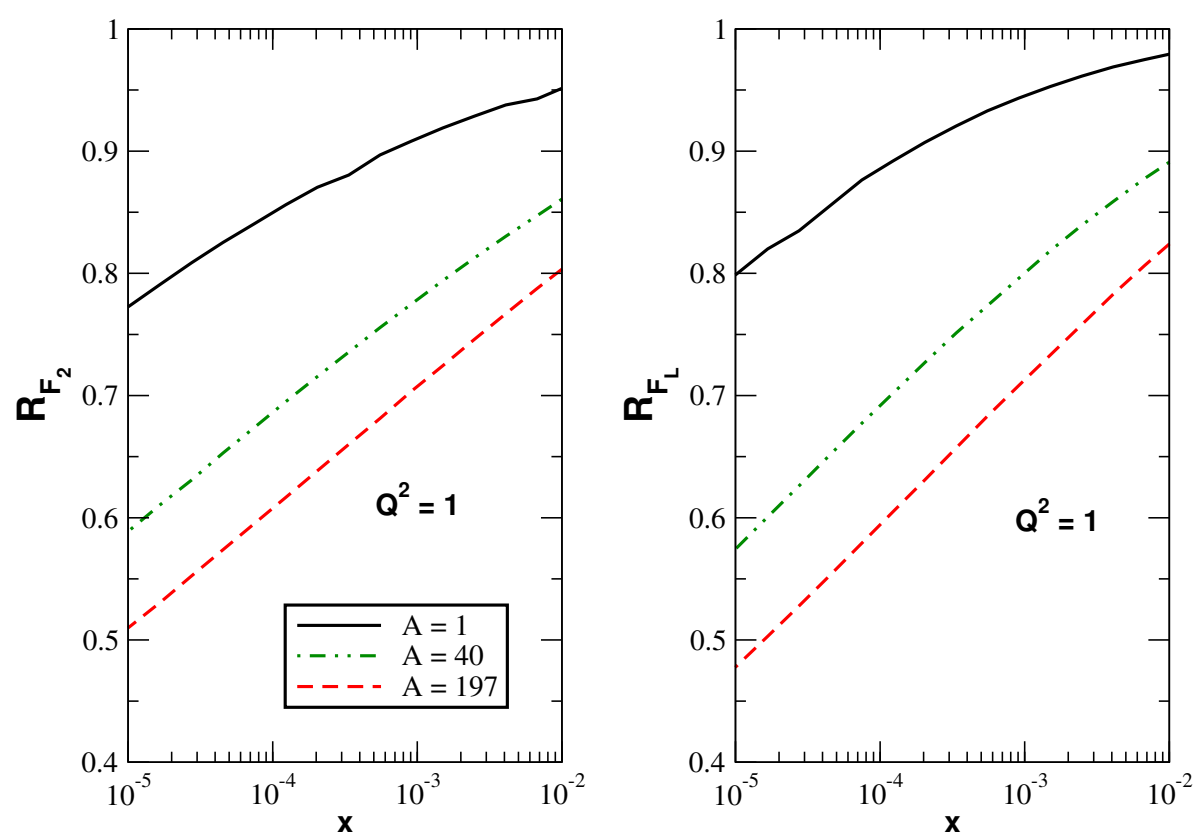

Figura 5.7: Razão entre as predições completa e linear para diferentes funções de estrutura nuclear.

grandes núcleos. Isso concorda com nosso resultado anterior, onde encontramos que o crescimento da função de estrutura nuclear em pequeno $x$ decresce para $A$ maior. Na figura 5.10(b) a dependência em $x$ do expoente para $Q^{2}=1 \mathrm{GeV}^{2}$ é mostrada. Neste caso apenas consideramos o modelo IIM e mostramos suas predições linear e completa. Vemos que as predições linear para $\lambda$ são similares, sendo quase independentes de $A$ e $x$. Por outro lado, os efeitos de saturação levam a uma diminuição de $\lambda$ em pequeno $x$, com esta diminuição sendo maior para $A=197$.

Nas figuras 5.11 e 5.12, mostramos a dependência de $x$ nas derivadas logarítmicas de $F_{2}$ e $F_{L}$ com respeito a $Q^{2}$. Essas derivadas, tanto quanto $\lambda$, foram calculadas numericamente. Vemos que ambas as derivadas têm comportamento semelhantes e as predições linear e completa, sendo idênticas para grande $x$. Para o slope $F_{2}$ e $A=1$, a diferença entre as predições linear e completa começa em $x \approx 10^{-4}$, aumentando em valores menores de $x$. Por outro lado, em $A=197$ ambas as predições diferem em valores menores de $x$ do que $\approx 10^{-2}$, com uma grande diferença entre as predições em pequeno $x$. Para o slope $F_{L}$ e $A=197$ temos um comportamento semelhante, mas com a diferença entre as predições começando em $x \approx 10^{-3}$. Em $A=1$ vemos 

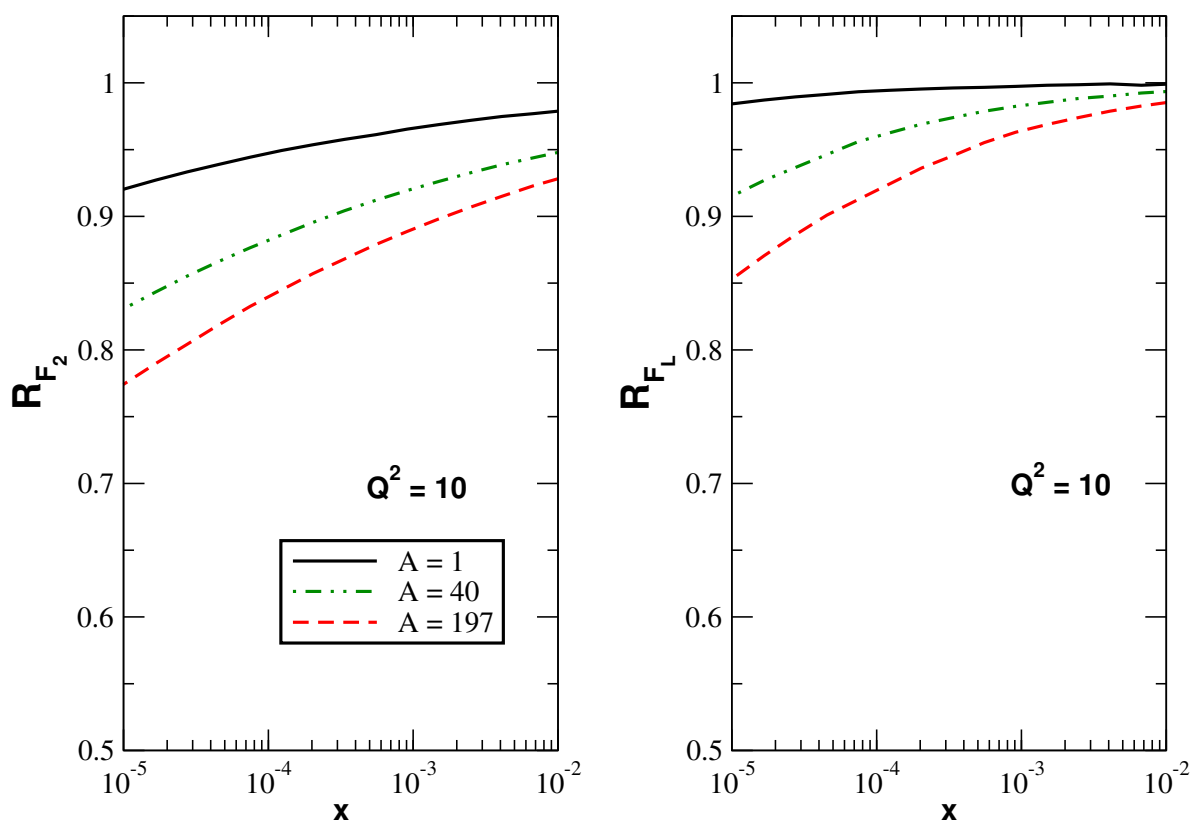

Figura 5.8: Razão entre as predições completa e linear para diferentes funções de estrutura nuclear.

que a predição completa é maior do que a linear para o alcance em $x$ da figura. Verificamos que em valores menores de $x$ a predição linear torna-se maior do que a completa. Finalmente, nas figuras 5.13 e 5.14, mostramos a dependência em $x$ das derivadas logarítmicas de $F_{2}$ e $F_{L}$ com respeito a $x$. Vemos que enquanto a predição linear cresce em pequeno x, a predição completa apresenta uma inclinação menor. Em particular, em $A=197$ estes observáveis são quase independentes de $x$ quando consideramos efeitos de saturação.

\subsubsection{A função de estrutura nuclear difrativa}

Processos difrativos em colisões $e A$ foram estudados nas referências $[67,68,69,70$, 71, 72, 73, 74, 75]. Motivados pelas referências [63, 76], analisamos aqui o comportamento da razão entre as funções de estrutura difrativas nucleares $R_{A 1, A 2}^{\text {diff }}\left(Q^{2}, \beta, x_{\mathbb{P}}\right)=$ $F_{2, A 1}^{D(3)} / F_{2, A 2}^{D(3)}$, onde $A_{1}$ e $A_{2}$ denotam o número atômico de dois núcleos distintos. Além disso vamos fazer predições mais detalhadas para as propriedades difrativas, tais como da função de estrutura difrativa $F_{2}^{D(3)}\left(Q^{2}, \beta, x_{\mathbb{P}}\right)$.

Para obtermos nossos resultados de difração usamos as expressões $(2,2.64),(2,2.69)$ e $(2,2.83)$ discutidas no Capítulo 2 e a substituição do parâmetro $B^{D}$ como feito na 

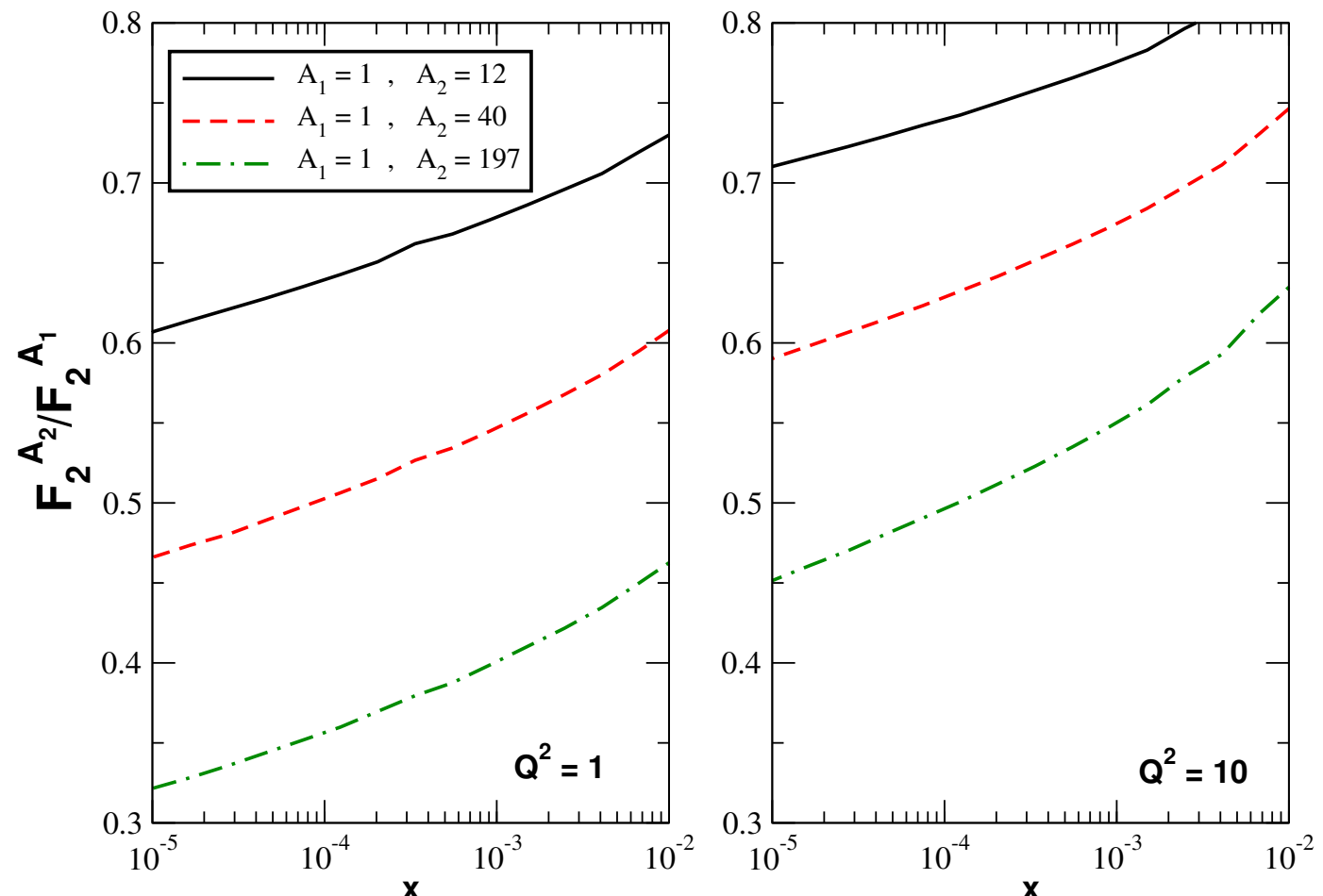

Figura 5.9: Razão entre as funções de estrutura nuclear como uma função de $x$ para diferentes valores de $A$ e fixo $Q^{2}=1$ e $10 \mathbf{G e V}^{2}$.

[77]:

$$
B_{A}=\frac{R_{A}^{2}}{4} \text { onde } R_{A}=1.2 A^{1 / 3} \mathrm{fm}
$$

Na figura 5.15 apresentamos nossas previsões para a função de estrutura difrativa $x_{\mathbb{P}} F_{2}^{D(3)}\left(x_{\mathbb{P}}, \beta, Q^{2}\right)$ como função de $\beta$ e para diferentes núcleos. Também mostramos a previsão linear para $x_{\mathbb{P}} F_{2}^{D(3)}$. É importante ressaltar que o Ansatz linear para a seção de choque de dipolo não descreve os dados de HERA. Entretanto, para estimarmos a importância da física de saturação e explicitar suas contribuições para diferentes regiões cinemáticas, uma comparação entre essas duas predições é válida. Podemos ver que a normalização de $x_{\mathbb{P}} F_{2}^{D(3)}$ é fortemente reduzida aumentando-se o número atômico, o que já é esperado de nossa análise da função peso. Além disso, embora a função de onda do fóton determine a estrutura geral do espectro em $\beta[34,26]$, a componente $q \bar{q} g$ que domina em regiões de pequeno $\beta$, tem seu comportamento mo- 

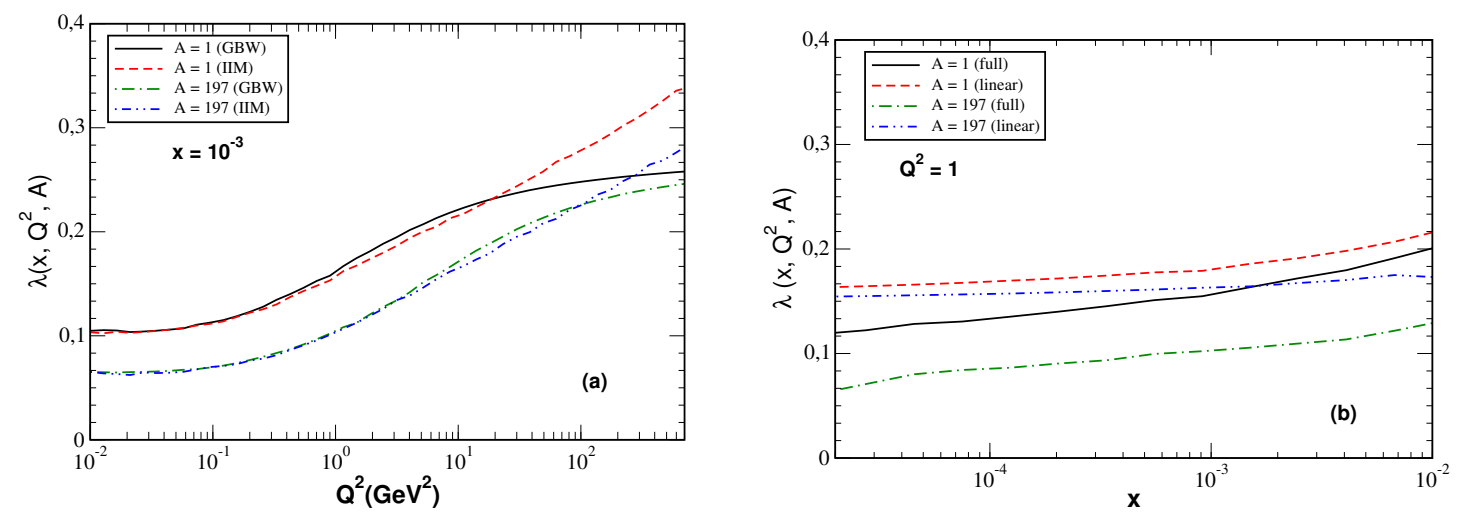

Figura 5.10: Expoente efetivo como uma função de: (a) $Q^{2}$ and (b) $x$.

dificado pelos efeitos de saturação e muda o comportamento de $x_{\mathbb{P}} F_{2}^{D(3)}$ nessa região. A função de estrutura difrativa torna-se quase plana na região de $\beta$ intermediário e grande $A$. Na figura 5.16 mostramos uma amplificação das curvas inferiores da figura 5.15 e incluímos também a componente $q \bar{q}_{T}$. Ao fazer isso, outra característica da difração em alvos nucleares emerge: a redução relativa da componente $q \bar{q} g$ com respeito a componente $q \bar{q}$.

Na figura 5.17, mostramos nossas previsões para $F_{2}^{D(3)}$, como uma função de $x_{\mathbb{P}} \mathrm{e}$ diferentes valores de $\beta, Q^{2}$ e $A$. Nossa escolha para a combinação de valores de $\beta$ e $Q^{2}$ foi motivada pelos resultados de HERA [78]. A dependência em $x_{\mathbb{P}}$ vem da seção de choque de dipolo que em nosso caso é dada pelo modelo IIM generalizado para alvos nucleares. Observamos que $x_{\mathbb{P}} F_{2}^{D(3)}$ aumenta em pequenos valores de $x_{\mathbb{P}}$. Entretanto, como a escala de saturação cresce com $A$, a dependência em $x_{\mathbb{P}}$ torna-se mais plana quando aumentamos o número atômico. Na figura 5.18, mostramos também nossas previsões para $F_{2}^{D(3)}$, mas agora comparando os modelos IIM e GBW.

Na figura 5.19 mostramos nossas predições para a razão

$$
R_{A 1, A 2}^{\text {diff }}\left(\beta, Q^{2}, x_{\mathbb{P}}\right)=\frac{F_{2, A 1}^{D(3)}\left(\beta, Q^{2}, x_{\mathbb{P}}\right)}{F_{2, A 2}^{D(3)}\left(\beta, Q^{2}, x_{\mathbb{P}}\right)}
$$

como função de $\beta$ e $x_{\mathbb{P}}$. Em nosso cálculo escolhemos $A_{2}=2$. Nossa análise é motivada pelas referências $[63,76]$. Nesses artigos foi sugerido que a dependência nuclear 

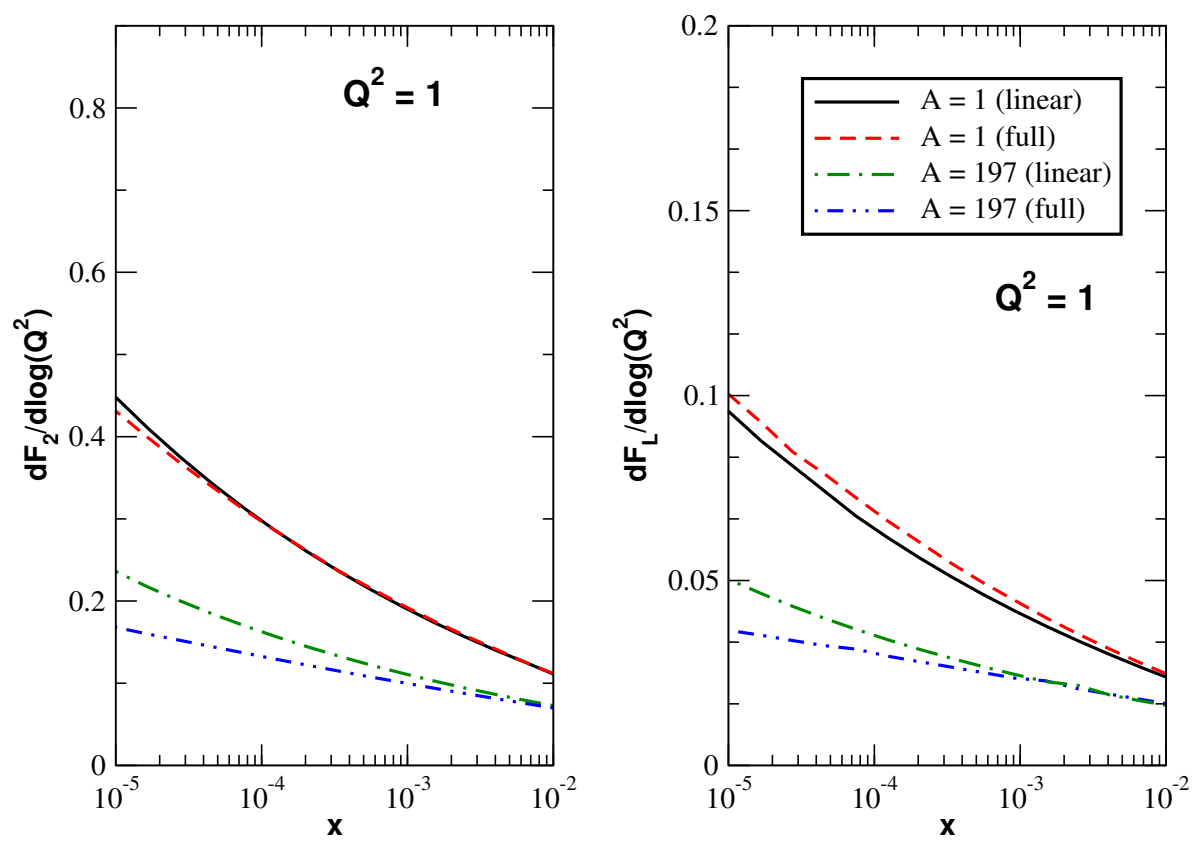

Figura 5.11: Derivada logarítmica das funções de estrutura total e longitudinal com respeito à $Q^{2}$.

desta razão poderia nos ajudar a estabelecer a universalidade da estrutura do Pomeron. Se $R_{A 1, A 2}^{\text {diff }}=1$ poderíamos concluir que a estrutura do Pomeron é universal. De nossa análise podemos antecipar que na representação de dipolo, assumindo a presença de efeitos de saturação, esta razão será dependente de $A$. Além disso, seu comportamento será determinado pela escala de saturação. Na figura 5.19 observamos um forte decréscimo de $R_{A 1, A 2}^{d i f f}$ como função de $A$. Ao mesmo tempo essa razão é independente de $x_{\mathbb{P}}$ e de $\beta$ em todos os valores de $A$. Para estimar o quanto esse comportamento é devido aos efeitos de saturação, calculamos a razão novamente usando apenas o termo linear da seção de choque de dipolo para o núcleo mais pesado $(A=197)$, para o qual os efeitos de saturação são dominantes, e mostramos nossos resultados na figura 5.20. Nas figuras 5.21 e 5.22, também mostramos as mesmas previsões, mas agora para núcleos diferentes. Como pode ser visto, a saturação é responsável pela fraca dependência da $R_{A 1, A 2}^{\text {diff }}$ em $x_{\mathbb{P}}$ e $\beta$. Nas referências $[63,76]$ a possibilidade de que a dependência em $A$ de $R_{A 1, A 2}^{\text {diff }}$ possa ser descrita pela razão das funções de estrutura nuclear foi sugerida. Verificamos a validade desta conjectura usando os resultados da referência [10] e concluímos que ela não é verdadeira, sendo a razão inclusiva maior do 

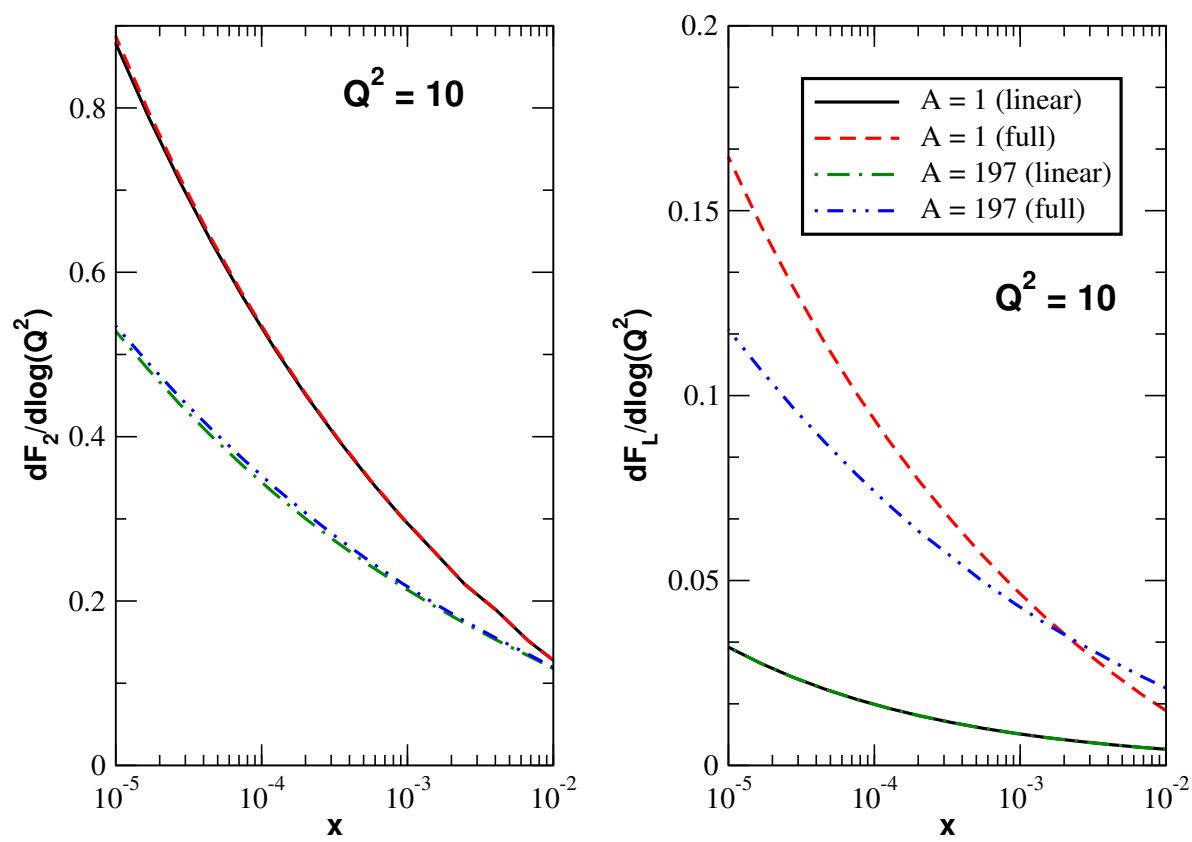

Figura 5.12: Derivada logarítmica das funções de estrutura total e longitudinal com respeito à $Q^{2}$.

que a difrativa.

\subsubsection{A razão $\sigma_{d i f f} / \sigma_{t o t}$}

Agora apresentamos uma análise qualitativa da dependência em $A$ da razão $\sigma_{d i f f} / \sigma_{\text {tot }}$ usando o modelo IIM generalizado para alvos nucleares. Seguindo a referência [26, 31] e assumindo que $\sigma_{d i p}$ no regime de saturação possa ser aproximado por $\sigma_{0}$, a parte transversal da seção de choque inclusiva e difrativa, na região cinemática onde $Q^{2}>$ $Q_{s}^{2}$, pode ser expressa como

$$
\begin{aligned}
\sigma_{T} \approx & \int_{0}^{4 / Q^{2}} \frac{d \boldsymbol{r}^{2}}{\boldsymbol{r}^{2}} \sigma_{0}\left[\frac{\boldsymbol{r}^{2} Q_{s}^{2}}{4}\right]^{\gamma_{e f f}}+\int_{\frac{4}{Q^{2}}}^{\frac{4}{Q_{s}^{2}}} \frac{d \boldsymbol{r}^{2}}{\boldsymbol{r}^{2}}\left(\frac{1}{Q^{2} \boldsymbol{r}^{2}}\right) \sigma_{0}\left[\frac{\boldsymbol{r}^{2} Q_{s}^{2}}{4}\right]^{\gamma_{e f f}} \\
& +\int_{4 / Q_{s}^{2}}^{\infty} \frac{d \boldsymbol{r}^{2}}{\boldsymbol{r}^{2}}\left(\frac{1}{Q^{2} \boldsymbol{r}^{2}}\right) \sigma_{0}
\end{aligned}
$$



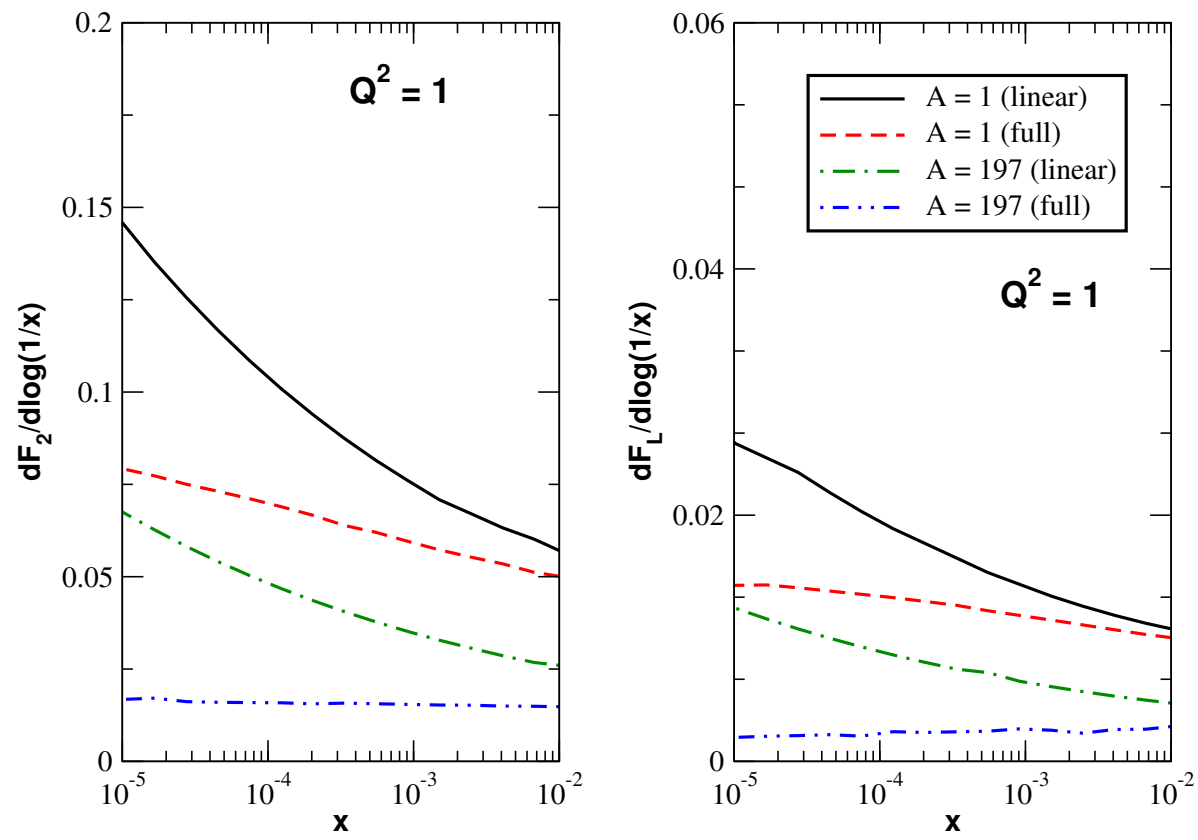

Figura 5.13: Derivada logarítmica das funções de estrutura total e longitudinal com respeito à $x$.

$\mathrm{e}$

$$
\begin{aligned}
\sigma_{T}^{D} \approx & \frac{1}{B_{A}}\left[\int_{0}^{4 / Q^{2}} \frac{d \boldsymbol{r}^{2}}{\boldsymbol{r}^{2}} \sigma_{0}^{2}\left[\frac{\boldsymbol{r}^{2} Q_{s}^{2}}{4}\right]^{2 \gamma_{e f f}}+\int_{\frac{4}{Q^{2}}}^{\frac{4}{Q_{s}^{2}}} \frac{d \boldsymbol{r}^{2}}{\boldsymbol{r}^{2}}\left(\frac{1}{Q^{2} \boldsymbol{r}^{2}}\right) \sigma_{0}^{2}\left[\frac{\boldsymbol{r}^{2} Q_{s}^{2}}{4}\right]^{2 \gamma_{e f f}}\right. \\
& \left.+\int_{4 / Q_{s}^{2}}^{\infty} \frac{d \boldsymbol{r}^{2}}{\boldsymbol{r}^{2}}\left(\frac{1}{Q^{2} \boldsymbol{r}^{2}}\right) \sigma_{0}^{2}\right] .
\end{aligned}
$$

Para obter uma expressão aproximada para essa razão, desprezaremos a dependência em $\boldsymbol{r}$ da dimensão anômala efetiva, ou seja, $\gamma_{\text {eff }}=\gamma=$ cte. Neste caso obtemos

$$
\sigma_{d i f f} / \sigma_{t o t} \approx\left[\frac{Q_{s}^{2}}{Q^{2}}\right]^{1-\gamma}
$$

Assumindo $\gamma=0.84$, como na referência [50], prevemos que a razão decresce com a virtualidade do fóton e apresenta uma dependência fraca na energia. Entretanto, analisando a dependência em $A$, esperamos um crescimento de aproximadamente 30 $\%$ quando $A$ vai de 2 to 197 . Na região cinemática onde $Q^{2}<Q_{s}^{2}$ a razão das seções 

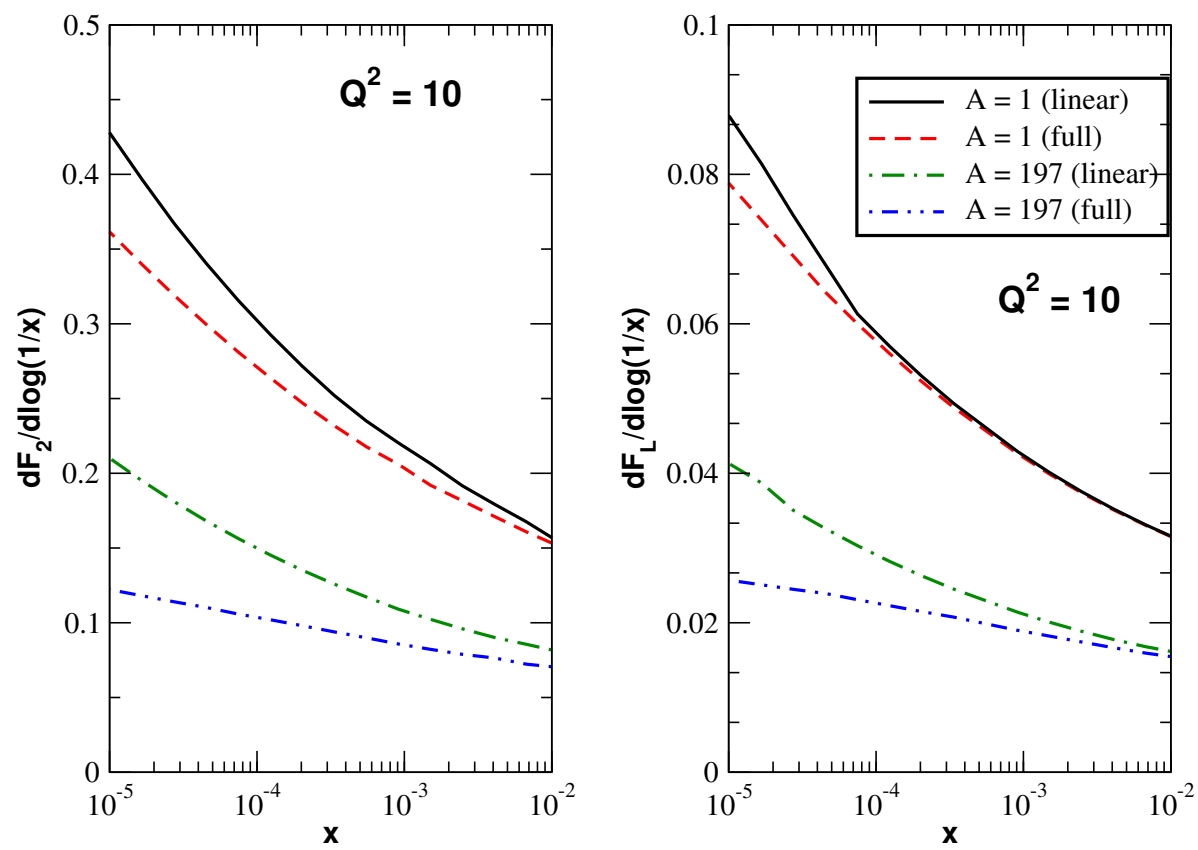

Figura 5.14: Derivada logarítmica das funções de estrutura total e longitudinal com respeito à $x$.

de choque apresenta um comportamento semelhante. A principal diferença é que no regime assintótico de energias muito altas, a seção de choque difrativa atinge o limite de disco negro de $50 \%$ da seção de choque total.

A razão $\sigma_{d i f f} / \sigma_{t o t}$ é calculada com as definições já vistas no Capítulo 2:

$$
\sigma_{t o t}=\sigma_{L, T}^{\gamma^{*} A}\left(x, Q^{2}\right)=\int d \alpha d^{2} \boldsymbol{r}\left|\psi_{L, T}(\alpha, \boldsymbol{r})\right|^{2} \sigma_{d i p}(x, \boldsymbol{r})
$$

$\mathrm{e}$

$$
\sigma_{\text {diff }}=\sigma_{T, L}^{D}=\left.\int_{-\infty}^{0} d t e^{B_{D} t} \frac{d \sigma_{T, L}^{D}}{d t}\right|_{t=0}=\left.\frac{1}{B_{D}} \frac{d \sigma_{T, L}^{D}}{d t}\right|_{t=0} .
$$

Na figura 5.23 mostramos $\sigma_{d i f f} / \sigma_{t o t}$ como função de $W$ e $x$ para diferentes valores de $A$. O limite de disco negro, $\sigma_{d i f f} / \sigma_{t o t}=1 / 2$, também é mostrado na figura 5.23. Vemos que a razão depende fracamente de $W$ e $x$, mas é suprimida fortemente para $Q^{2}$ crescente. Isso sugere que, na região profundamente perturbativa a difração será mais suprimida. Esse mesmo comportamento foi observado nos dados difrativos de $e p$ [78]. Além disso, a dependência na energia da razão é muito fraca, aumentando com 

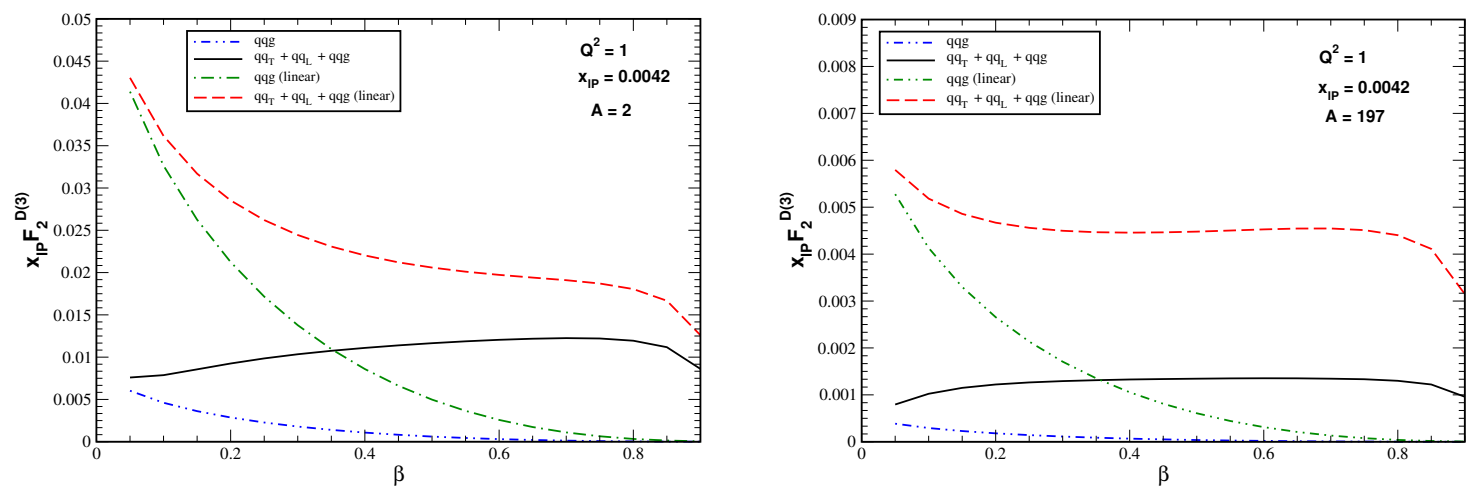

Figura 5.15: Função de estrutura difrativa $F_{2}^{D(3)}$ como função de $\beta$ e núcleos distintos. A componente $q \bar{q} g$ da função de estrutura difrativa é explicitamente mostrada.
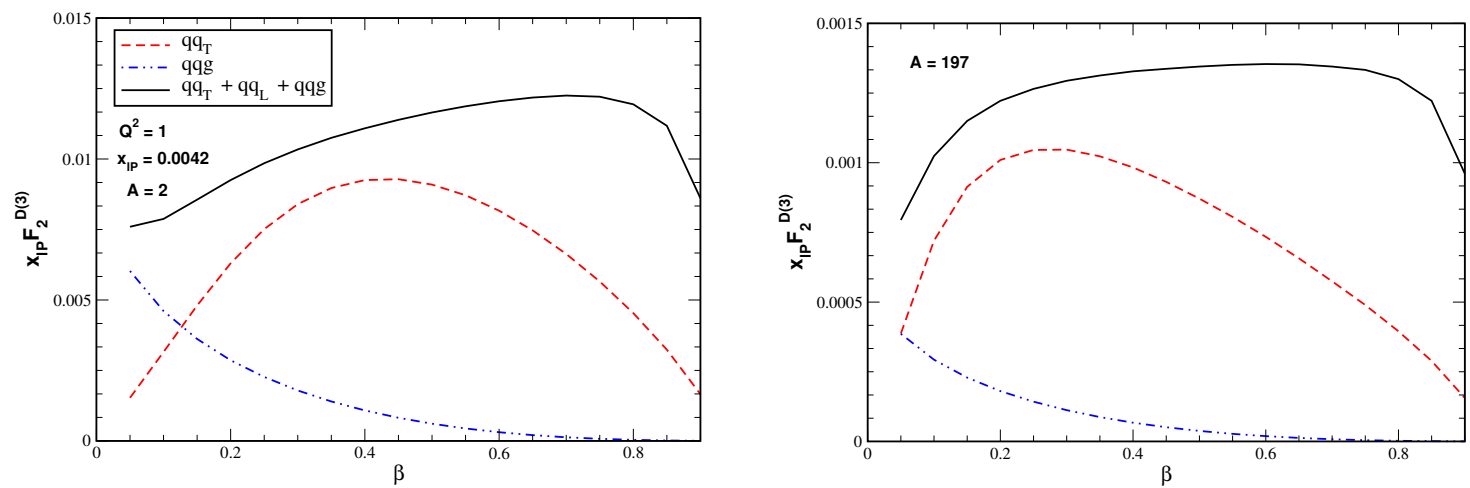

Figura 5.16: Função de estrutura difrativa $F_{2}^{D(3)}$ como função de $\beta$ e núcleos distintos. As componentes transversal e $q \bar{q} g$ da função de estrutura difrativa são explicitamente mostrada.

$A$, tornando-se $37 \%$ (30 \%) maior para ouro em comparação com próton (deuteron). Este comportamento concorda qualitativamente com os cálculos anteriores de [71] e com nossas estimativas anteriores. O aparecimento de um grande gap de rapidez em $37 \%$ de todos os eventos de espalhamento $e A$ seria uma confirmação da saturação. 


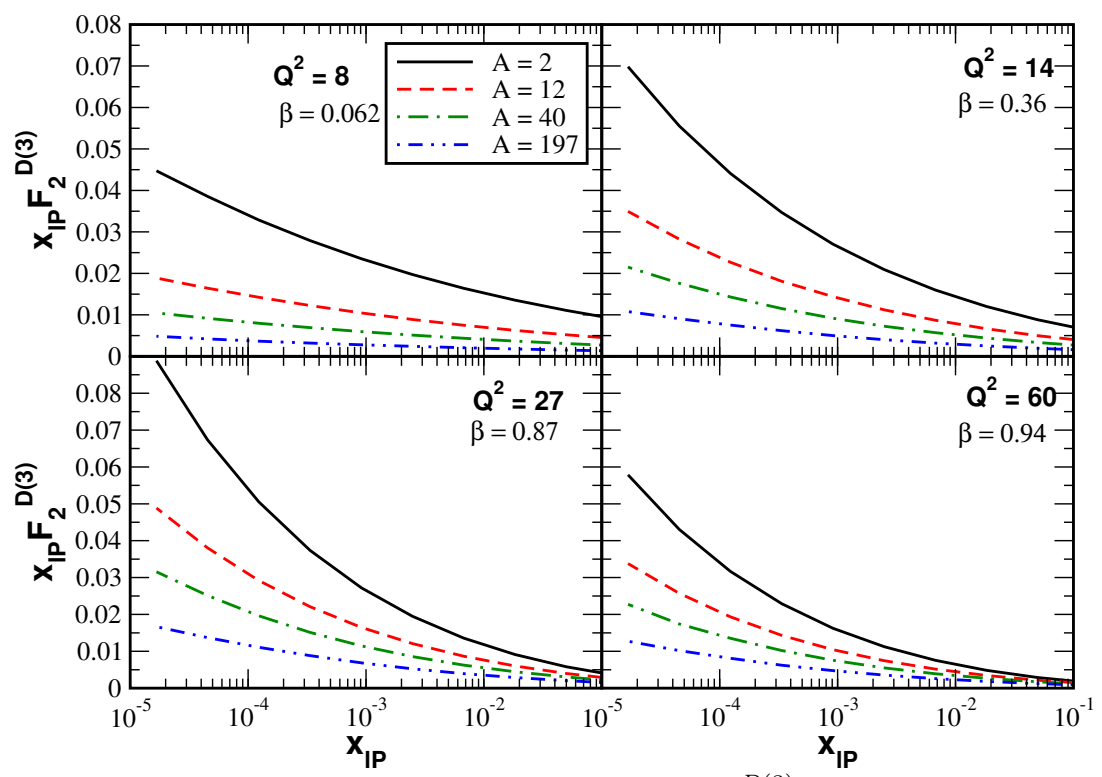

Figura 5.17: Predições para a funções de estrutura difrativa $x_{\mathbb{P}} F_{2}^{D(3)}\left(x_{\mathbb{P}}, \beta, Q^{2}\right)$ como função de $x_{\mathbb{P}}$ para diferentes valores de $\beta, Q^{2}$ e $A$.

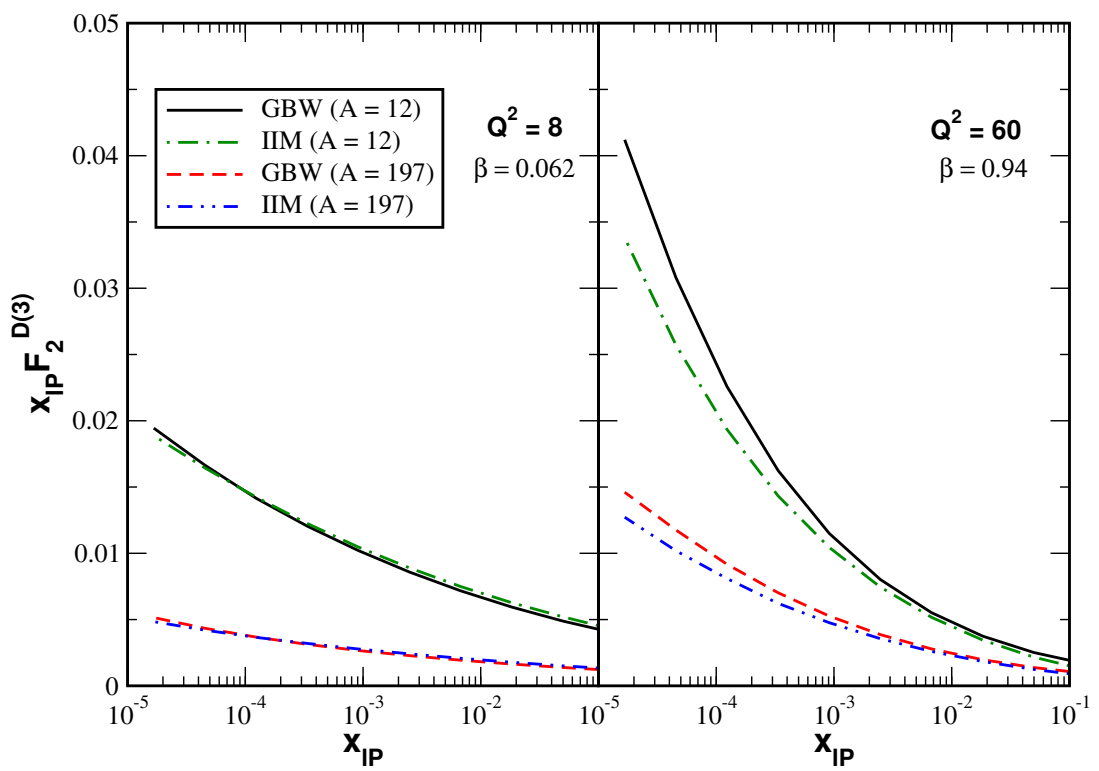

Figura 5.18: Predições para a funções de estrutura difrativa $x_{\mathbb{P}} F_{2}^{D(3)}\left(x_{\mathbb{P}}, \beta, Q^{2}\right)$ como função de $x_{\mathbb{P}}$ para diferentes valores de $\beta, Q^{2}$ e $A$, para dois modelos diferentes. 

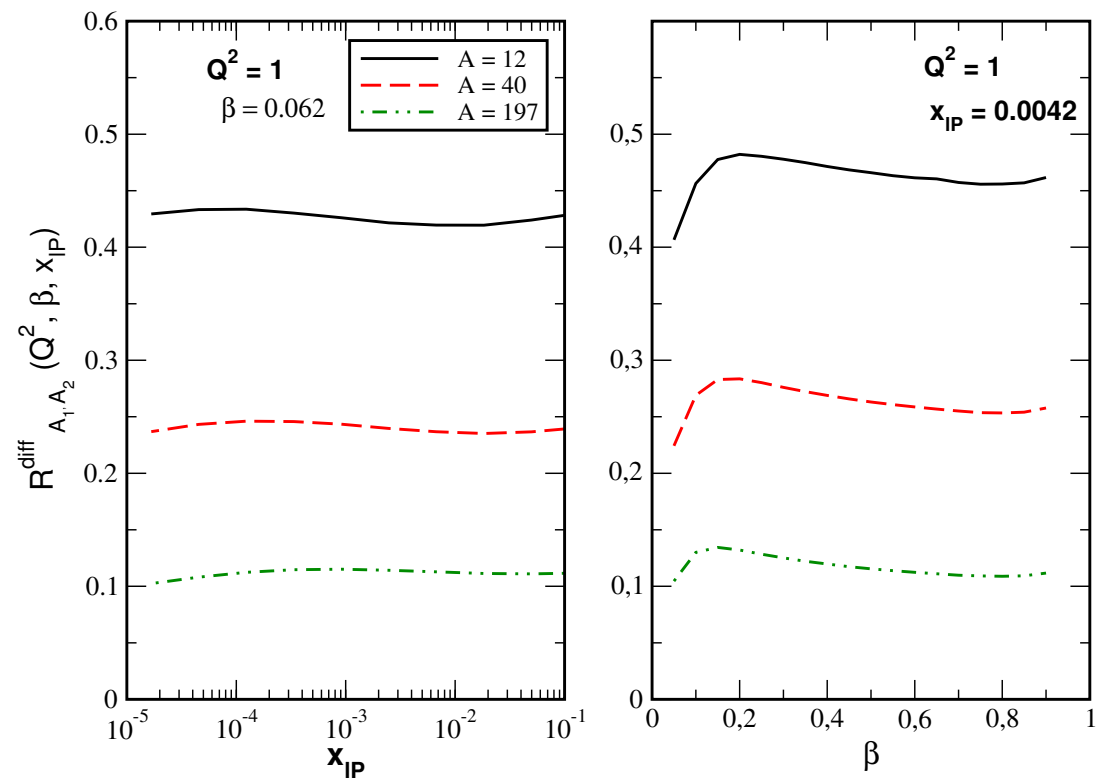

Figura 5.19: $\boldsymbol{A}$ razão $R_{A 1, A 2}^{\text {diff }}\left(Q^{2}, \beta, x_{\mathbb{P}}\right)=\frac{F_{2, A 1}^{D(3)}\left(Q^{2}, \beta, x_{\mathbb{P}}\right)}{F_{2, A 2}^{D(3)}\left(Q^{2}, \beta, x_{\mathbb{P}}\right)}$ como função de $x_{\mathbb{P}}$ e $\beta$. Comparação entre as predições para a razão em diferentes valores de $A_{1}$.
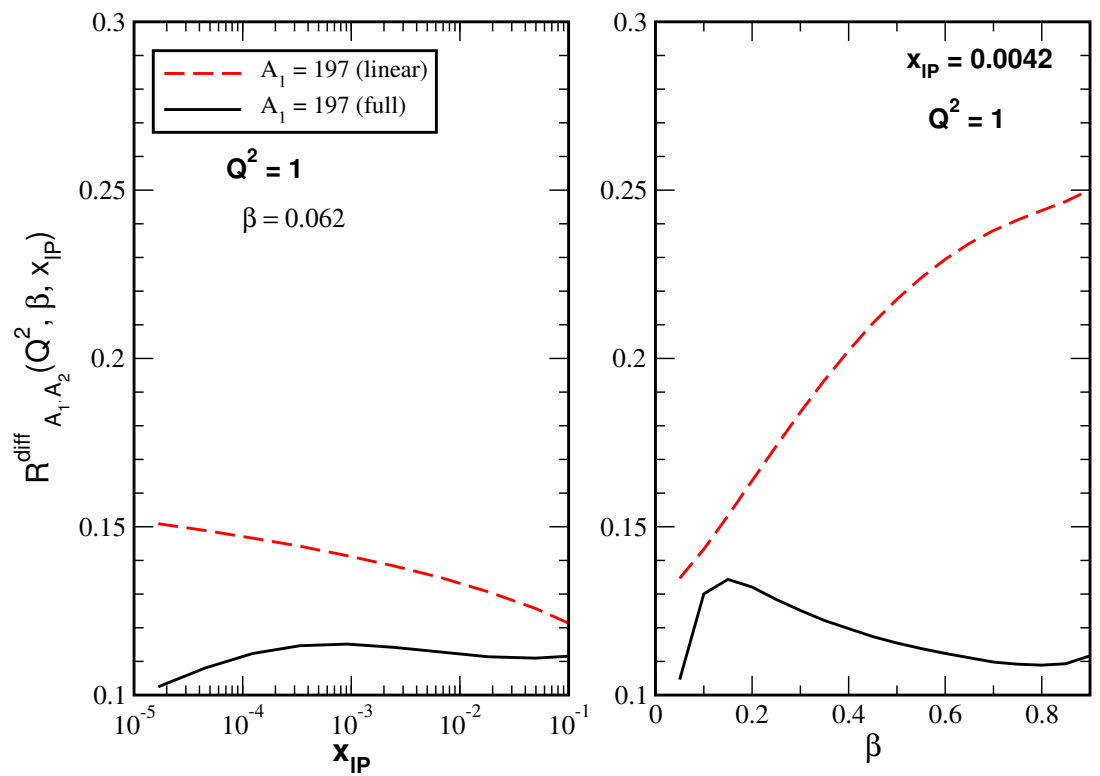

Figura 5.20: $\boldsymbol{A}$ razão $R_{A 1, A 2}^{\text {diff }}\left(Q^{2}, \beta, x_{\mathbb{P}}\right)=\frac{F_{2, A 1}^{D(3)}\left(Q^{2}, \beta, x_{\mathbb{P}}\right)}{F_{2, A 2}^{D(3)}\left(Q^{2}, \beta, x_{\mathbb{P}}\right)}$ como função de $x_{\mathbb{P}}$ e $\beta$. Comparação entre as previsões completa e linear. 


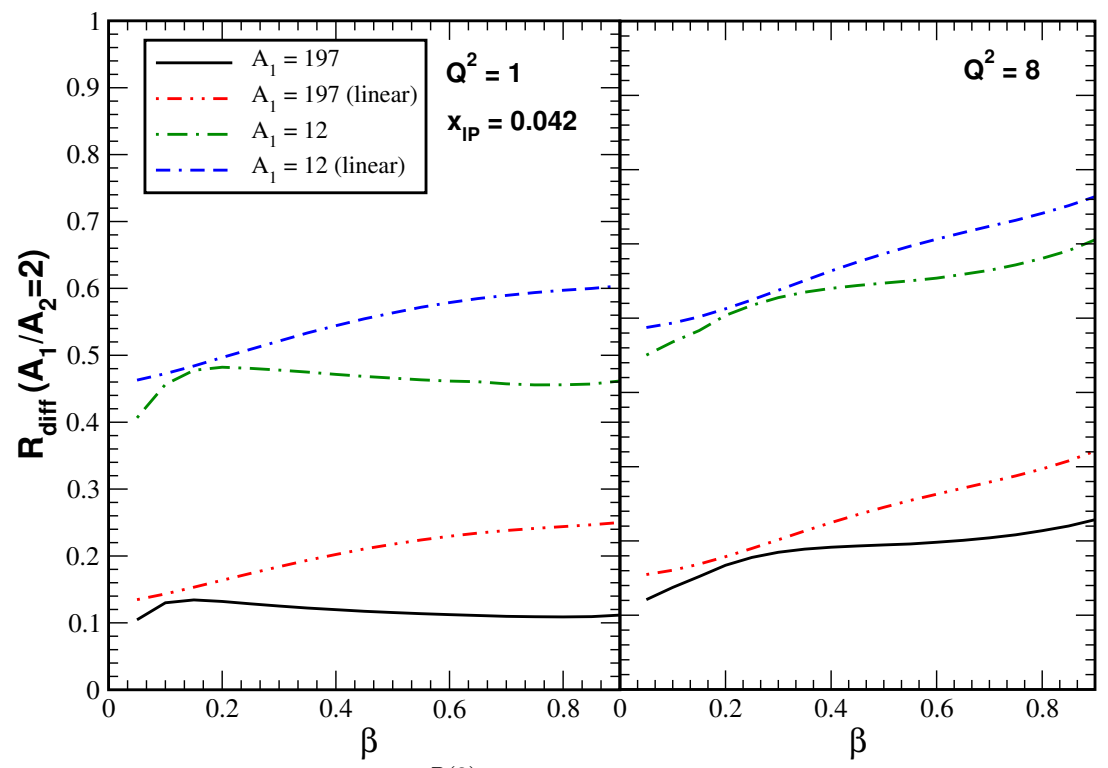

Figura 5.21: A razão $R_{A 1, A 2}^{\text {diff }}\left(Q^{2}, \beta, x_{\mathbb{P}}\right)=\frac{F_{2, A 1}^{D(3)}\left(Q^{2}, \beta, x_{\mathbb{P}}\right)}{F_{2, A 2}^{D(3)}\left(Q^{2}, \beta, x_{\mathbb{P}}\right)}$ como função de $x_{\mathbb{P}}$ e $\beta$. Comparação entre as previsões completa e linear.

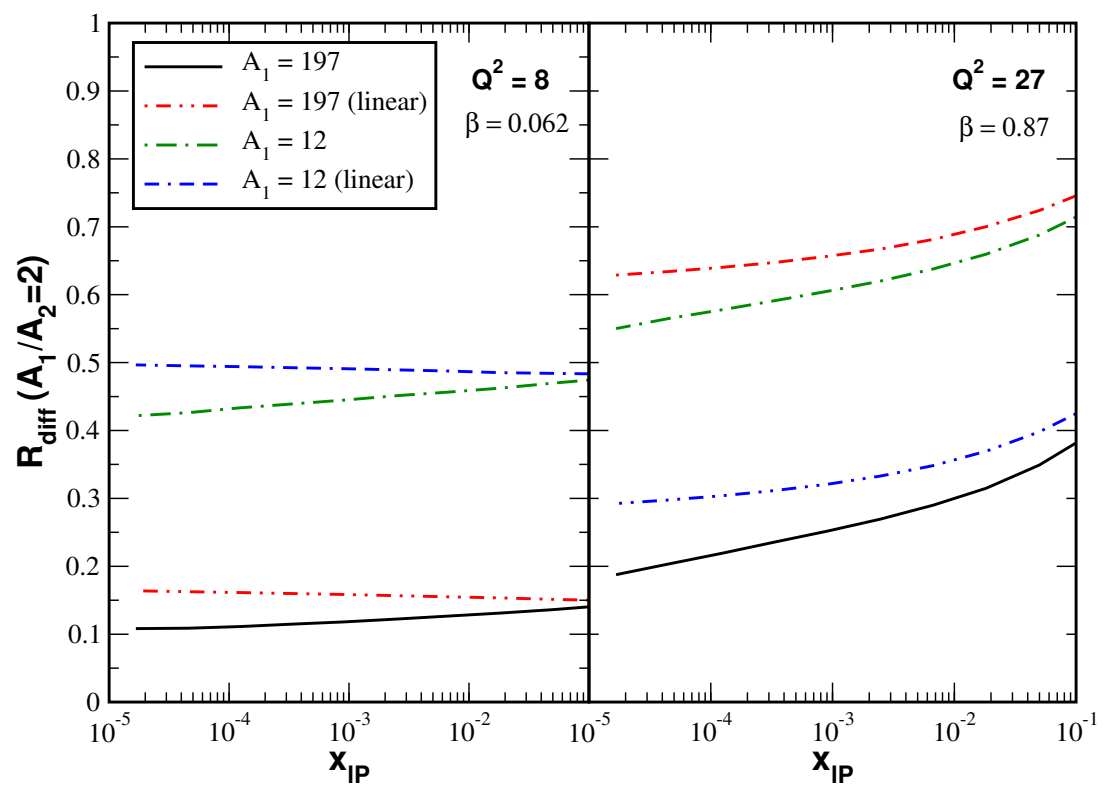

Figura 5.22: A razão $R_{A 1, A 2}^{\text {diff }}\left(Q^{2}, \beta, x_{\mathbb{P}}\right)=\frac{F_{2, A 1}^{D(3)}\left(Q^{2}, \beta, x_{\mathbb{P}}\right)}{F_{2, A 2}^{D(3)}\left(Q^{2}, \beta, x_{\mathbb{P}}\right)}$ como função de $x_{\mathbb{P}}$ e $\beta$. Comparação entre as previsões completa e linear. 

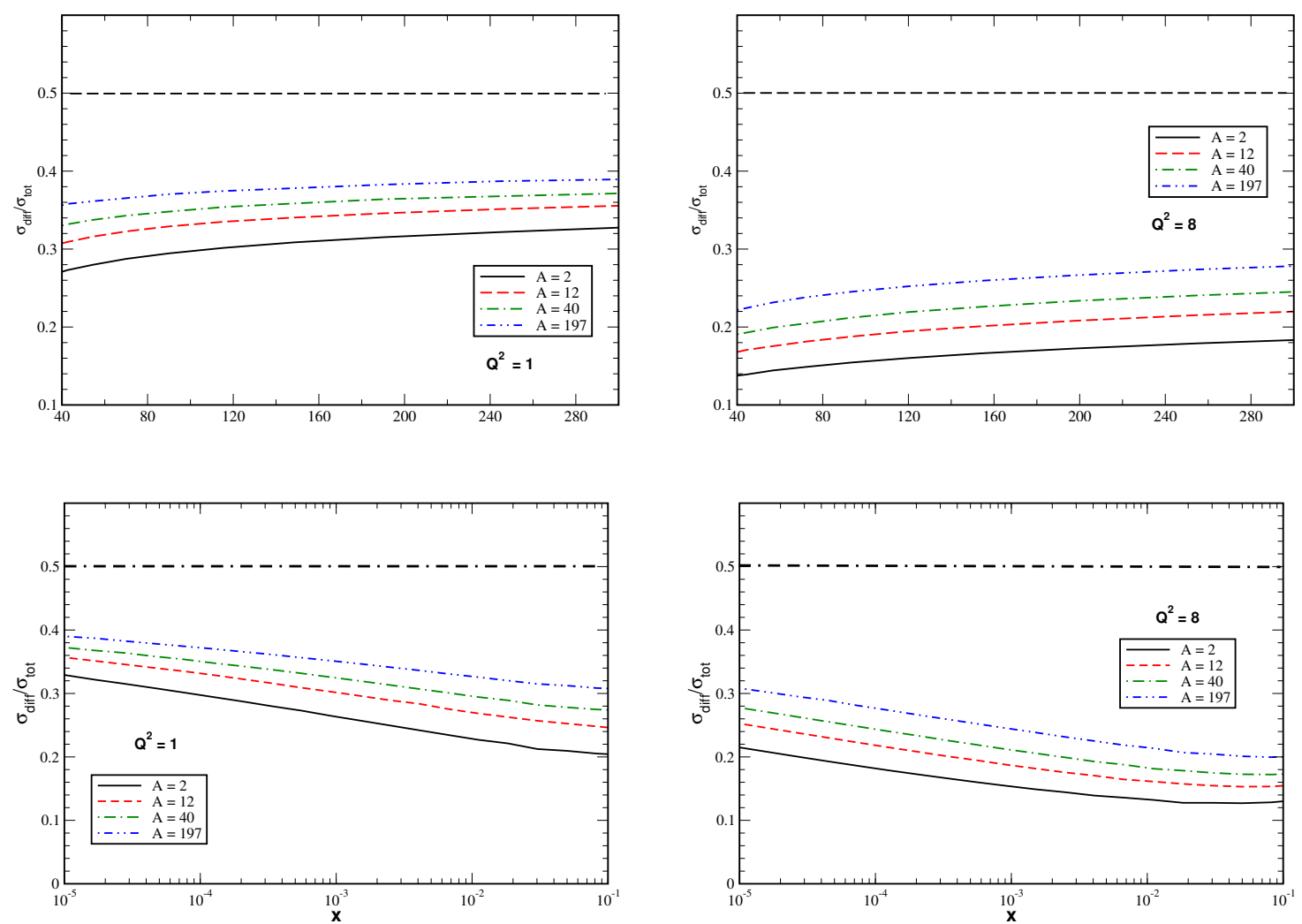

Figura 5.23: $A$ razão entre as seções de choque difrativa e total como função de $x$ e $W$ para diferentes valores de $A$ e $Q^{2}$. O limite de disco negro, $\sigma_{d i f f} / \sigma_{t o t}=1 / 2$, também é mostrado. 


\section{Capítulo 6}

\section{Interação quarkonium-hádron a altas}

\section{energias}

Neste capítulo apresentamos uma aplicação do modelo de dipolos ao estudo de campos cromo-elétricos e a interação quarkonium-hádron. Em comum aos capítulos anteriores, temos a idéia de que o dipolo de cor segue uma trajetória reta e permanece inalterado durante a interação com um campo semi-clássico.

Os resultados apresentados neste capítulo estão publicados na referência $[1,2]$.

\subsection{Introdução}

As seções de choque quarkonium-hádron $\left(\sigma_{\Phi h}\right)$ são ferramentas necessárias para entender os dados da produção de quarkonium no RHIC. Nos últimos seis anos, muitos esforços foram colocados neste problema [79] e progressos foram alcançados, especialmente no que diz respeito à seção de choque em baixa energia, próxima do limiar de dissociação. Na região de energia longe deste limiar a situação é menos clara e a dependência na energia é ainda objeto de debate. A extrapolação do cálculo válido em baixa energia aponta para diferentes direções. Resultados obtidos com o modelo de quarks não relativístico [80] indicam uma queda abrupta da seção de choque. Esse comportamento é devido ao comportamento gaussiano da função de onda dos quark 
usada no modelo de troca de quarks. Este mesmo comportamento pode ser encontrado na abordagem de Lagrangianas quirais com a introdução de fatores de forma dependentes da energia [81]. Em regras de soma da QCD [82] a seção de choque é monototicamente crescente com a energia.

Os cálculos de $\sigma_{\Phi h}$ feitos para serem válidos em alta energia $(\sqrt{s} \simeq 20 \mathrm{GeV})$ são escassos: a aproximação de Bhanot-Peskin (BP) [83, 84, 85, 86, 87, 88], QCD perturbativa mais a extrapolação geométrica [89], o modelo do vácuo estocástico (MSV) [90] e o formalismo de dipolo no cone de luz [91]. Durante os últimos anos a aproximação de ordem dominante de BP foi usada com muita frequência. Entretanto, os cálculos mais recentes de próxima ordem dominante apresentados em [88] mostram que para o charmonium, o formalismo falha, pois este sistema não é suficientemente pesado. A maioria dos cálculos mencionados acima prevêe um crescimento da seção de choque. $\mathrm{Na}$ referência [87], $\sigma_{\Phi h}$ cai com a energia e na referência [90] fica constante.

Se o quarkonium for tratado como um hádron ordinário, sua seção de choque de interação com qualquer outro hádron ordinário deverá crescer suavemente em energias maiores, do mesmo modo como as seções de choque próton-próton ou píon-próton. A razão para isso é o aumento da importância da dinâmica da QCD perturbativa e a manifestação da natureza partônica de todos os hádrons. Entretanto, esta natureza partônica começa a ser dominante apenas em energias muita altas $(\sqrt{s}>100 \mathrm{GeV})$. $\mathrm{Na}$ região de energia relevante para a física do RHIC aspectos não perturbativos são ainda muito importantes. Nos cálculo de alta energia mencionado acima, diferentes ingredientes não perturbativos foram empregados: momentos da distribuição de glúons no hádron [83, 84, 85, 86, 87, 88]; as funções de onda do hádron e do quarkonium [89] e o valores esperados do vácuo da QCD (condensados) [90].

Como ainda existe discrepâncias no que concerne aos números (os quais podem variar de uma ordem de magnitude em diferentes estimativas) e ao comportamento com a energia, achamos que é interessante calcular $\sigma_{\Phi h}$ com uma abordagem não perturbativa, colocando ênfase na regra imposta pelos campos cromoelétricos. Em [92] um tratamento semelhante foi adotado para estudar a dissociação do quarkonium 
dentro de um plasma da QCD. Os campos elétricos de cor que aparecem no elemento de matriz de transição foram relacionados com a densidade de carga de cor do meio e foram calculados em um modelo específico do Plasma de Quarks e Glúons (QGP). Começaremos com uma expressão semelhante para a amplitude de transição, mas como estamos numa fase puramente hadrônica, devemos conhecer o campo cromoelétrico dentro de núcleons e píons. Existem progressos no estudo desses campos, vindo de modelos do vácuo da QCD [93], da QCD na rede [94], do Método dos Correlatores de Campos (FCM) [95] e da QCD no gauge de Coulomb [96]. Esperamos nos beneficiar desses avanços e usar as formas aproximadas dos campos cromoelétricos obtidas nesses trabalhos em nosso problema. Para isso, trataremos a interação entre o quarkonium e o hádron como sendo análoga à interação de um pequeno dipolo atravessando um grande capacitor e interagindo com os campos elétricos de cor, mas não com suas fontes. Na parte final desse capítulo, discutiremos a validade desta hipótese. Usando uma interação de contato entre um quark pesado (ou antiquark) e um quark (ou antiquark) calculamos a seção de choque correspondente. O modelo desenvolvido por nós, têm algumas semelhanças com a aproximação de BP, mas é muito mais simples. Algumas hipóteses simplificadoras foram usadas para manter o cálculo quase-analítico e preservar o entendimento da física básica.

\section{$6.2 \mathrm{O}$ modelo}

\subsubsection{A Hamiltoniana de interação}

O ponto de partida é assumir que o quarkonium (dipolo) é pequeno comparado com o hádron (capacitor). Como consequência, o par $\bar{Q}-Q$ interage com o campo externo de cor, mas não com suas fontes (quarks). Além disso, o campo externo de cor tem apenas componentes de baixo momento ("soft glúons") e deste modo é capaz de transferir apenas uma pequena quantidade de energia, a qual será suficiente para dissociar o estado ligado. No caso do charmonium, a energia de ligação típica é $\epsilon \simeq 0.6 \mathrm{GeV}$. 
Assim, numa primeira aproximação

$$
\epsilon \ll M_{\Phi}
$$

onde $M_{\Phi}$ é a massa do estado ligado $\left(M_{\Phi} \simeq 3 \mathrm{GeV}\right)$. No caso do bottonium essa aproximação é melhor. A energia de ligação é também pequena comparada a energia da colisão,

$$
\epsilon \ll \sqrt{s}
$$

A aproximação $(6,2.1)$ justifica o uso da teoria de perturbação da mecânica quântica (a aproximação de Born) e a aproximação $(6,2.2)$ justifica o uso da aproximação eikonal, a qual, neste caso, implica que o hádron segue uma trajetória reta e permanece inalterado durante a interação. Na figura 6.1, mostramos a representação do espalhamento e nossa escolha de coordenadas, no referencial de repouso do quarkonium: $\vec{r}_{1}$ e $\overrightarrow{r_{2}}$ são as coordenadas do quark e antiquark e $\vec{E}^{a}$ é o campo cromoelétrico no projétil, que será um próton ou um píon, movendo-se com uma velocidade constante $\vec{v}$ e com um parâmetro de impacto $\vec{b}$.

Com estas hipóteses podemos escrever a hamiltoniana de interação como:

$$
H_{\text {int }}=g\left(T_{1}^{a} \vec{E}_{1}^{a} \vec{r}_{1}+\bar{T}_{2}^{b} \vec{E}_{2}^{b} \vec{r}_{2}\right)
$$

onde $T^{a}\left(\bar{T}^{b}\right)$ são os geradores do grupo de cor SU(3) na representação fundamental (conjugada); $\vec{E}_{1}^{a}$ e $\vec{E}_{2}^{b}$ são os campos cromoelétricos gerados pelo hádron em movimento (capacitor) e sentidos pelo quark e antiquark no estado ligado, respectivamente. Eles serão transformados (por Lorentz) para o referencial de repouso do quarkonium, trazendo para nosso cálculo o fator gama de Lorentz, que será a origem da dependência na energia $(\sqrt{s})$ de nossos resultados. Iremos negligenciar a componente magnética, uma vez que ela não faz qualquer trabalho sobre as cargas e deste modo não é efetiva na transferência de energia. Além disso, a interação magnética é inversamente proporcional à massa do quark, sendo desse modo suprimida. Podemos representar esse 


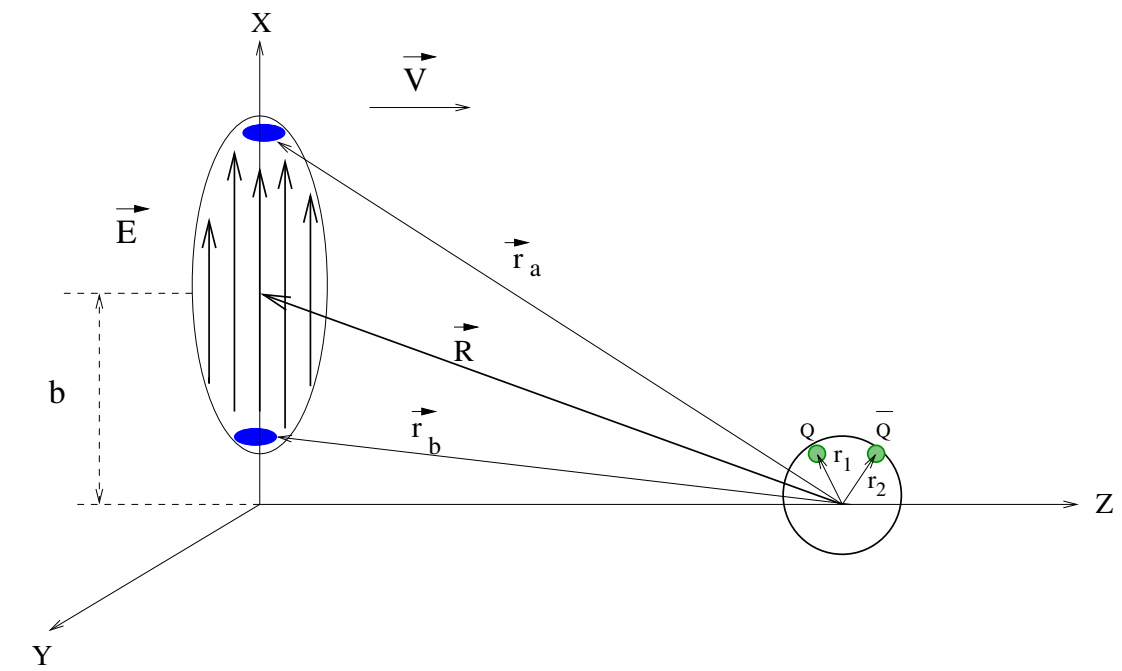

Figura 6.1: Interação Quarkonium-hádron no referencial de repouso do quarkonium. O hádron é um capacitor de cor que move-se para direita. $O$ campo $\vec{E}^{a}$ está ao longo da direção $x$. Os pontos negros "gordos" representam as "placas" do capacitor: quark-antiquark para o pion e quark-diquark para o próton.

campo externo por:

$$
\vec{E}^{a}\left(r_{e}, t\right)=\gamma \vec{E}_{0}^{a} \exp \left(-\frac{\left(X-x_{e}\right)^{2}}{d^{2}}-\frac{\left(Y-y_{e}\right)^{2}}{d^{2}}\right) \exp \left(-\gamma^{2} \frac{\left[v t-z_{e}\right]^{2}}{d^{2}}\right)(6
$$

com $e=1,2$. X, Y e Z são as coordenadas do hádron e $\gamma$ é o fator de Lorentz usual. $Z=v t$, pois o hádron move-se com velocidade $\vec{v}$ ao longo do eixo $z . \vec{E}_{0}^{a}$, será abreviado por $E$, e é o campo elétrico de cor no centro do projétil. O raio quadrático médio do projétil está relacionado ao parâmetro $d$ por

$$
\sqrt{\left\langle r_{h}^{2}\right\rangle}=0.86 d
$$

Desprezamos a perturbação na trajetória do hádron, pois estamos estudando reações no regime de alta energia e não perturbativo, ou seja, com baixa transferência de momento. $X$ e $Y$ estão relacionados ao parâmetro de impacto $b$ por $b^{2}=X^{2}+Y^{2}$. Note que por simplicidade, escolhemos uma direção preferencial para o campo, neste caso, o eixo $x$. 
Desprezando o movimento do centro de massa (c.m.), reescrevemos $(6,2.3)$ como

$$
H_{\text {int }}=g\left(\frac{\lambda^{a}}{2} E_{1}^{a}+\frac{\lambda^{b^{T}}}{2} E_{2}^{b}\right)\left(\frac{x_{1}-x_{2}}{2}\right)
$$

Também por simplicidade, quando trabalhamos com (6,2.5), tomaremos $x_{1}-x_{2} \simeq a$, onde $a$ é a separação típica entre o quark e o antiquark. Inicialmente, o par quarkantiquark está em uma região localizada do espaço.

\subsubsection{O estado inicial}

A função de onda tem a parte espacial e de cor definida por:

$$
\Psi_{i}=f\left(r_{1}, r_{2}\right) c_{n} d_{n}
$$

onde $c_{n}$ e $d_{n}$, com $n=1,2,3$, são os vetores de cor inicial [97] para o quark e antiquark respectivamente, tomado no estado singleto de cor. Escolhemos

$$
f\left(r_{1}, r_{2}\right)=N_{i} \exp \left[-\frac{\vec{r}_{1}^{2}}{a^{2}}\right] \exp \left[-\frac{\vec{r}_{2}^{2}}{a^{2}}\right] \exp \left(-i \varepsilon_{i} t\right)
$$

onde $\varepsilon_{i}\left(\varepsilon_{i}=M_{\Phi}\right)$ é a energia inicial do quarkonium e $N_{i}$ é uma constante de normalização dada por:

$$
N_{i}^{2}=\left(\frac{2}{\pi}\right)^{3} \frac{1}{a^{6}}
$$

A função de onda inicial $\Psi_{i}$ descreve o confinamento dos quarks e também a liberdade assintótica, pois ela permite que o quarks sejam independentes dentro da sacola. É fácil ver que a conexão entre o raio quadrático médio do quarkonium e o parâmetro $a$ é

$$
\sqrt{\left\langle r_{Q \bar{Q}}^{2}\right\rangle}=1.09 a
$$




\subsubsection{O estado final}

Sob a ação do campo externo a função de onda inicial $\Psi_{i}$ vai para o estado final $\Psi_{f}$ dado por:

$$
\Psi_{f}=t\left(r_{1}, r_{2}\right) c_{j} d_{k}
$$

onde $c_{j}$ e $d_{k}$, com $j, k=1,2,3$, são os vetores de cor finais do quark e do antiquark e $t\left(r_{1}, r_{2}\right)$ é a parte espacial da função de onda final. No estado final desta reação temos que lidar com a transição de um par de quark e antiquark excitado para um par de mésons $D-\bar{D}$ (ou $B-\bar{B})$. Esta transição é altamente não perturbativa e terá que ser modelada. Escolhemos duas aproximações.

\section{Modelo $A$}

Primeiro assumimos que o par quark e antiquark é convertido em dois mésons livres (um $M$ e um $\bar{M}$ ), os quais são descritos por ondas planas

$$
t_{A}\left(r_{1}, r_{2}\right)=N_{A} \exp \left(i \vec{p}_{1} \cdot \vec{r}_{1}\right) \exp \left(i \vec{p}_{2} \cdot \vec{r}_{2}\right) \exp \left(-i \varepsilon_{f} t\right)
$$

onde $\vec{p}_{1}$ e $\vec{p}_{2}$ são os momentos do méson e $N_{A}$ é a constante de normalização dada por:

$$
N_{A}^{2}=\frac{1}{V^{2}}
$$

com $V$ sendo um volume de normalização arbitrário que será cancelado no cálculo da seção de choque. Na equação $(6,2.9) \varepsilon_{f}$ é a energia final do par $Q-\bar{Q}$. A energia transferida durante da reação será suficiente para dissociar o estado ligado em um par de mésons $D \bar{D}$ ou $B \bar{B}$, e portanto

$$
\varepsilon_{f}=\sqrt{\left(\vec{p}_{1}\right)^{2}+m_{M}^{2}}+\sqrt{\left(\vec{p}_{2}\right)^{2}+m_{\bar{M}}^{2}}
$$

onde $m_{M}\left(m_{\bar{M}}\right)$ é a massa resultante da fragmentação do quark (antiquark). Com esta 
definição de $\varepsilon_{f}$ deixamos implícito a conversão de quarks em hádrons, um processo que não pode ser bem descrito neste modelo simples.

As hipóteses $(6,2.9)$ e $(6,2.10)$ são razoáveis, mas elas representam um caso de "extrema liberdade": elas não levam em conta a perda de energia do quark pai, quando este é convertido em um méson final (menos energético). Este processo é descrito, em certas situações, pela função de fragmentação. Além disso, os mésons finais podem ter qualquer momento, e embora os momentos maiores sejam naturalmente suprimidos nos cálculos, estamos superestimando o espaço de fase da reação.

\section{Modelo $B$}

Como alterntiva a $(6,2.9)$ e $(6,2.10)$ usaremos uma segunda aproximção mais conservadora para o estado final. Assumiremos que a energia transferida para o quarkonium pesado $\Phi$, o transformará em um estado excitado $\Phi^{\prime}$ (mas ainda ligado). A massa desse estado ligado será um pouco maior do que a primeira excitação do charmonium e do bottonium, $\Psi^{\prime}$ e $\Upsilon^{\prime}$ respectivamente. É sabido que essas excitações são fracamente ligadas. Através da escolha de massas um pouco maiores para eles, que estão acima do limite dos decaimentos em $D-\bar{D}$ e em $B-\bar{B}$, estaremos simulando um processo de fragmentação em um par de mésons quase parados. Essa hipótese é complementar à $(6,2.9)$ e $(6,2.10)$ uma vez que aqui estamos dando aos quarks pesados uma "liberdade mínima". A função de onda do estado fundamental foi escolhida Gaussiana $(6,2.7)$. Inspirados no oscilador harmônico, escolhemos a função de onda do primeiro estado excitado como uma função que é ímpar na direção $x$ (a direção do campo cromoelétrico) e simétrica em $x_{1}$ e $x_{2}$ :

$$
t_{B}\left(r_{1}, r_{2}\right)=N_{B} \frac{x_{1}+x_{2}}{2} \exp \left[-\frac{\vec{r}_{1}^{2}}{{a^{\prime 2}}^{2}}\right] \exp \left[-\frac{{\overrightarrow{r_{2}}}^{2}}{{a^{\prime}}^{2}}\right] \exp \left(-i \varepsilon_{f} t\right)
$$


onde a constante de normalização é dada por:

$$
N_{B}^{2}=\left(\frac{4}{\pi}\right)^{3} \frac{1}{a^{\prime 8}}
$$

e $a^{\prime}$ é o tamanho do estado $\Psi^{\prime}$ ou $\Upsilon^{\prime}$. Usando a função de onda $(6,2.11)$ temos algumas vantagens. Em primeiro lugar, isso evita a definição do mecanismo de fragmentação com a introdução de novos parâmetros. Em segundo lugar, como pode ser visto, $(6,2.11)$ é ortogonal à $(6,2.9)$, tal que o elemento de matriz $\left\langle\Psi_{f}\left|H_{\text {int }}\right| \Psi_{i}>\right.$ é zero se a hamiltoniana for constante. Isto não ocorre quando usamos $(6,2.9)$ e portanto a aproximação $A$ (modelo $A$ ) poderá conter contribuições espúrias. O mesmo comentário cabe para o cálculo feito em referência [92]. Isso torna a comparação entre as aproximações $A$ e $B$ mais necessário. Finalmente, vamos usar a hamiltoniana $(6,2.3)$, sem a aproximação, $x_{1}-x_{2} \simeq a$, feita no modelo $A$.

\section{Amplitude de transição e seções de choque}

A amplitude de transição para o modelo $A$ pode ser facilmente calculada de $(6,2.5)$, $(6,2.4),(6,2.6),(6,2.8)$ e $(6,2.9)$ :

$$
T_{f i}=\left\langle\Psi_{f}\left|H_{i n t}\right| \Psi_{i}\right\rangle=\int d t \int d^{3} \vec{r}_{1} \int d^{3} \vec{r}_{2} \Psi_{f}^{*}\left(\vec{r}_{1}, \vec{r}_{2}\right) H_{i n t}\left(\vec{r}_{1}, \vec{r}_{2}\right) \Psi_{i}\left(\vec{r}_{1}, \vec{r}_{2}\right)
$$

Uma expressão análoga vale para o modelo $B$ com o uso de $(6,2.3),(6,2.4),(6,2.6)$, $(6,2.8)$ e $(6,2.11)$. O próximo passo é tomar a amplitude quadrada $\left|T_{f i}\right|^{2}=T_{f i}^{*} T_{f i}$, e como a cor não é observada, tomamos a média de todos os estados iniciais de cor e somamos sobre os estados finais:

$$
\left|T_{f i}\right|^{2} \rightarrow \overline{\left|T_{f i}\right|^{2}} \equiv \frac{1}{3} \sum_{n} \frac{1}{8} \sum_{a} \sum_{j} \sum_{k}\left|T_{f i}\right|^{2}
$$


A seção de choque com o modelo $A$ é dada por:

$$
\sigma_{A}=\int \frac{V}{\left(2 \pi^{3}\right)} d^{3} p_{1} \int \frac{V}{\left(2 \pi^{3}\right)} d^{3} p_{2} 2 \pi \int_{0}^{\infty} d b b \overline{\left|T_{f i}\right|^{2}}
$$

Essa expressão é muito simples e pode ser calculada quase analiticamente. Devido ao ansatz gaussiano $(6,2.4)$ e $(6,2.6)$ podemos facilmente integrar $(6,2.12)$ nas coordenadas e no parâmetro de impacto. No último passo de (6,2.14), a integração sob o espaço de fase foi feita numericamente. Em [2] fizemos a hipótese adicional de que os mésons que saem estão quase em repouso e poderemos desse modo simplificar $(6,2.10)$ e calcular a integração sob $\vec{p}_{1}$ e $\vec{p}_{2}$ analiticamente. Aqui preferimos ser mais "exatos" e calcular a última integral numericamente.

A seção de choque com o modelo $B$ é simplesmente dada por:

$$
\sigma_{B}=2 \pi \int_{0}^{\infty} d b b \overline{\left|T_{f i}\right|^{2}}
$$

a qual, depois de substituições e integrações resulta:

$$
\sigma_{B}=\frac{32}{3} \pi^{5}\left\langle g E_{0}\right\rangle^{2} \frac{\gamma^{2}}{\gamma^{2}-1} \frac{d^{10} a^{\prime 8} a^{10}}{\left(a^{\prime 2}+a^{2}\right)^{5}\left[a^{\prime 2} a^{2}+d^{2}\left(a^{\prime 2}+a^{2}\right)\right]^{3}} \exp \left(-\omega^{2} \frac{\frac{\gamma^{2} a^{2} a^{2}}{\left(a^{\prime 2}+a^{2}\right)}+d^{2}}{2\left(\gamma^{2}-1\right)}\right)
$$

onde

$$
\omega=\varepsilon_{f}-\varepsilon_{i}=M_{\Phi^{\prime}}-M_{\Phi}
$$

Da equação $(6,2.16)$ vemos que a seção de choque aumenta com a energia $(\gamma)$ e satura em um valor constante. A magnitude do campo cromoelétrico é controlada pela contração de Lorentz do projétil. Com relação aos parâmetros de tamanho, $a, a^{\prime}$ e $d$, a seção de choque primeiro cresce e então cai com o aumento dos valores desses parâmetros. Os valores de intensidade máxima dependem do modelo e podem mudar para uma escolha diferente de função de onda. Entretanto, a imagem física é muito simples. A expressão $(6,2.16)$ nos diz que a probabilidade de converter o quarkonium com um dado tamanho inicial $a$ para um estado final de tamanho $a^{\prime}$, tende a zero se 
$a^{\prime}=0$, ou se $a^{\prime} \rightarrow \infty$, porque o "overlap"entre esses diferentes estados e o estado inicial é zero. Pela mesma razão, a seção de choque se anula para $a=0$ e para $a \rightarrow \infty$. O parâmetro $d$ está associado com a extensão do capacitor. No limite em que $d \rightarrow \infty$, a dependência espacial do potencial desaparece e torna-se uma constante e então $<\Psi_{f}\left|H_{\text {int }}\right| \Psi_{i}>\rightarrow<\Psi_{f} \mid \Psi_{i}>=0$.

\subsubsection{A interação com as fontes}

$\mathrm{Na}$ introdução assumimos que o quarkonium está bem representado por um dipolo pequeno, que atravessa um grande capacitor. Entretanto, isso pode ser uma hipótese muito forte, pois o dipolo não é sempre tão pequeno. Por exemplo, quando comparamos o tamanho do charmonium com o tamanho do píon temos tipicamente que $\frac{a}{d} \simeq \frac{0.4}{0.6} \simeq 0.67$. Portanto, se faz necessário incluir a interação entre quark e antiquark no quarkonium com as fontes (as "placas" do capacitor) que podem ser um quark ou um antiquark no caso do píon, ou um quark e um diquark no caso do próton.

Para levar essas interações em conta, assumimos que a interação entre um quark (ou diquark) do capacitor e um quark charme (ou antiquark) do dipolo possa ser dividida em uma parte de curta distância e outra de longa distância. A última delas já foi incluída na interação com os campos cromoelétricos produzidos pelas fontes.

\section{Modelo $C$}

A interação de curtas distâncias pode ser aproximada pela parte da interação de contato (aquela que contém a função delta) do potencial da troca de um glúon [98]:

$$
H_{i n t}=V_{O G E}=\sum_{i=a, b} \sum_{j=1,2} \frac{\alpha_{s}}{4} \overrightarrow{\lambda_{i}} \cdot \overrightarrow{\lambda_{j}}\left(\frac{1}{r_{i j}}-\frac{2 \pi}{3 m_{i} m_{j}} \overrightarrow{\sigma_{i}} \cdot \overrightarrow{\sigma_{j}} \delta^{3}\left(\overrightarrow{r_{i j}}\right)\right)
$$

onde $\lambda$ e $\sigma$ são as matrizes de Gell-Mann e de Pauli respectivamente, que são responsáveis pelas interações de cor e de spin. O termo de Coulomb na expressão acima será desprezado, pois ele é um termo de longo alcance. Os índices $i=a, b$ e $j=1,2$ 
referem-se às partículas no capacitor e no dipolo respectivamente. Com esta notação, na interação entre as partículas $a$ e 1, a função delta toma a forma:

$$
\delta^{3}\left(\overrightarrow{r_{a}}-\overrightarrow{r_{1}}\right)=\delta\left(x_{a}-x_{1}\right) \times \delta\left(y_{a}-y_{1}\right) \times \delta\left(z_{a}-z_{1}\right)
$$

onde $\overrightarrow{r_{1}}=\left(x_{1}, y_{1}, z_{1}\right)$ é a mesma de antes e $\overrightarrow{r_{a}}=\left(x_{a}, y_{a}, z_{a}\right)$ é a coordenada da partícula $a$ no referencial de repouso do quarkonium. Para calcular a amplitude de transição precisamos conhecer a nova função de onda, que agora inclui ambos quarkonium e capacitor. Elas são

$$
\Psi_{i}=f\left(\overrightarrow{r_{1}}, \overrightarrow{r_{2}}\right) g\left(\overrightarrow{r_{a}}, \overrightarrow{r_{b}}\right) c_{n} d_{n} e_{m} h_{m}
$$

$\mathrm{e}$

$$
\Psi_{f}=t_{C}\left(\overrightarrow{r_{1}}, \overrightarrow{r_{2}}\right) g\left(\overrightarrow{r_{a}}, \overrightarrow{r_{b}}\right) c_{i} d_{j} e_{l} h_{k}
$$

Na equação $(6,2.20)$ a função $f$ é a mesma de antes e é dada pela equação $(6,2.7)$. A função $t_{C}$ representa a distribuição espacial dos quarks pesados no estado final, a qual assumimos ser um estado excitado, mas ainda ligado, como no modelo $B$. Entretanto se escolhessemos $t_{C}=t_{B}$, a amplitude de transição $<\Psi_{f}\left|H_{\text {int }}\right| \Psi_{i}>$ se anularia devida a interação de contato não depender das coordenadas e consequentemente como, < $\Psi_{f} \mid \Psi_{i}>$ é o produto de uma função ímpar por uma par de $x$, sendo deste modo nulo. Uma vez que estamos mais interessados em saber a ordem de magnitude desta interação de contato, aproximaremos a função de onda do estado final por uma gaussiana, dada por:

$$
t_{C}\left(\overrightarrow{r_{1}}, \overrightarrow{r_{2}}\right)=N_{C} \exp \left(\frac{-r_{1}^{2}}{a^{\prime 2}}\right) \exp \left(\frac{-r_{2}^{2}}{a^{\prime 2}}\right) e^{-i \varepsilon_{f} t}
$$

com a constante de normalização dada por:

$$
N_{C}^{2}=\left(\frac{2}{\pi}\right)^{3} \frac{1}{a^{\prime 6}} .
$$

O cálculo da interação de contato requer o conhecimento da posição dos quarks no 
capacitor, que será dado pela função $g$

$$
\begin{aligned}
g\left(\overrightarrow{r_{b}}, \overrightarrow{r_{b}}\right) & =N_{P} \exp \left[\frac{-\left(x_{a}-X\right)^{2}}{d^{2}}\right] \exp \left[\frac{-\left(y_{a}-Y\right)^{2}}{d^{2}}\right] \\
& \times \exp \left[\frac{-\left(x_{b}-X\right)^{2}}{d^{2}}\right] \exp \left[\frac{-\left(y_{b}-Y\right)^{2}}{d^{2}}\right] \\
& \times \exp \left[\frac{-\gamma^{2}\left(z_{a}-Z\right)^{2}}{d^{2}}\right] \exp \left[\frac{-\gamma^{2}\left(z_{b}-Z\right)^{2}}{d^{2}}\right]
\end{aligned}
$$

onde $Z=v t, d$ e $\gamma$ tem o mesmo significado anterior, e $N_{P}$ é a constante de normalização da função de onda do projétil, dada por:

$$
N_{P}^{2}=\frac{8 \gamma^{2}}{\pi^{3} d^{6}}
$$

Note que $g$ é a mesma no estado inicial e no final. Esta hipótese é consistente com a aproximação eikonal anteriormente introduzida e evita a introdução de novos parâmetros.

Com esses ingredientes, podemos calcular a amplitude de transição,

$$
\begin{aligned}
T_{f i}= & \left\langle\Psi_{f}\left|H_{i n t}\right| \Psi_{i}\right\rangle \\
= & \int d t \int d^{3} \vec{r}_{1} d^{3} \overrightarrow{r_{2}} \int d^{3} \overrightarrow{r_{a}} d^{3} \overrightarrow{r_{b}} \Psi_{f}^{*}\left(\overrightarrow{r_{1}}, \overrightarrow{r_{2}}, \overrightarrow{r_{a}}, \overrightarrow{r_{b}}\right) \\
& H_{\text {int }}\left(\overrightarrow{r_{1}}, \overrightarrow{r_{2}}, \overrightarrow{r_{a}}, \overrightarrow{r_{b}}\right) \Psi_{i}\left(\overrightarrow{r_{1}}, \overrightarrow{r_{2}}, \overrightarrow{r_{a}}, \overrightarrow{r_{b}}\right)
\end{aligned}
$$

e a seção de choque,

$$
\begin{aligned}
\sigma_{C} & =\frac{2^{10}}{3^{4}} \pi \alpha_{s}^{2}\left(\frac{1}{m_{a} m_{1}}+\frac{1}{m_{a} m_{2}}+\frac{1}{m_{b} m_{1}}+\frac{1}{m_{b} m_{2}}\right)^{2} \\
& \times \frac{\gamma^{6} a^{\prime 6}}{\gamma^{2}-1} \frac{\left.a^{\prime 2}+a^{2}\right)^{5}\left[d^{2}\left(a^{\prime 2}+a^{2}\right)+2 a^{2} a^{\prime 2}\right]}{\left(a^{2}\right.} \\
& \times \exp \left(-\omega^{2} \frac{\frac{\gamma^{2} a^{\prime 2} a^{2}}{\left(a^{\prime 2}+a^{2}\right)}+d^{2}}{4\left(\gamma^{2}-1\right)}\right)
\end{aligned}
$$

onde usamos $(6,2.13)$ e a expressão análoga para a soma e a média sobre os spins. Além de um fator numérico, $(6,2.16)$ e $(6,2.27)$ tem a mesma dependência em ener- 
gia. Isto ocorre, pois a mesma contração de Lorentz no expoente da hamiltoniana $(6,2.3)$ e $(6,2.4)$ levada para $(6,2.16)$, está agora presente na função de onda do capacitor $(6,2.24)$. Além disso, o mesmo fator $\gamma$ de Lorentz, anteriormente multiplicando

o campo $\vec{E}^{a}$ em $(6,2.4)$, reaparece agora na constante de normalização $(6,2.25)$. A dependência de $(6,2.27)$ em $a$ e $a^{\prime}$ é agora qualitativamente a mesma encontrada em $(6,2.16)$, tendo a mesma origem física. Finalmente a seção de choque na equação $(6,2.27)$ é agora uma função que decresce monototicamente com $d$. O comportamento observado com $d$ significa que, em um capacitor grande, os quarks estão espalhados numa área transversal maior e eles tornam-se mais difícies de ser encontrados pelos quarks charme do alvo e sofrer uma interação de contato.

\subsection{Resultados e Discussão}

Nas estimativas numéricas apresentadas a seguir, adotaremos $d=0.8$ e $0.6 \mathrm{fm}$ para o próton e o píon respectivamente. Tomaremos também $a=0.4$ e $0.2 \mathrm{fm}$ para $J / \psi$ e $\Upsilon$ respectivamente, e $a^{\prime}=0.8$ e $0.45 \mathrm{fm}$ para $\Psi^{\prime}$ e $\Upsilon^{\prime}$. O estado ligado $\Psi\left(m_{\Psi}=3.07\right.$ $\mathrm{GeV})$ e $\Upsilon\left(m_{\Upsilon}=9.46 \mathrm{GeV}\right)$ será, no modelo $A$, dissociado em um par de mésons $D$ $\left(m_{D}=1.87 \mathrm{GeV}\right)$ e $B\left(m_{B}=5.27 \mathrm{GeV}\right)$. Os estados excitados usados nos modelos $B$ e $C$ têm massas $m_{\Phi^{\prime}}=3.8 \mathrm{GeV}$ and $m_{\Phi^{\prime}}=11 \mathrm{GeV}$ no caso do charmonium e bottonium respectivamente. O valor da constante de acoplamento forte e das massas dos quarks constituintes são as mesmas usadas em [98], ou seja, $\alpha_{s}=0.64, m_{q}=0.3$ $\mathrm{GeV}, m_{c}=1.2 \mathrm{GeV}, m_{b}=4.74 \mathrm{GeV}$ e a massa do diquark é $m_{d}=0.60 \mathrm{GeV}$.

Como está claro de $(6,2.5)$ e $(6,2.4)$, precisamos conhecer o valor médio do campo cromoelétrico no projétil $g E=\langle h|g E| h\rangle$. Numa primeira aproximação esse número poderá ser identificado com a tensão na corda $\kappa \simeq 0.18 \mathrm{GeV}^{2}$ ou $\kappa \simeq 0.9 \mathrm{GeV} / \mathrm{fm}$. A tensão na corda calculada em [96] é algumas vezes maior. Em [95] o perfil transversal da corda foi estudado. A intensidade de $\langle h|g E| h\rangle$ depende da separação quark-antiquark (ou quark-diquark), sendo maior para sistemas maiores e foi calculada apenas para sistemas grandes. Assim $\langle h|g E| h\rangle$ é outra fonte de diferenças entre os projéteis próton 
e píon. Fazendo uma média dos valores encontrados em [95], escolhemos $\langle h|g E| h\rangle=1$ $\mathrm{GeV} / \mathrm{fm}$.

Como mencionado na introdução, nosso modelo têm aspectos comuns com a aproximação de BP. Assim, devemos comparar nossos resultados para $\sigma_{\Phi h}$ com aqueles obtidos em [85]:

$$
\sigma_{\Phi h}=2.5\left(1-\frac{\lambda_{0}}{\lambda}\right)^{6.5} \mathrm{mb}
$$

com $\lambda$ dado por

$$
\lambda \simeq \frac{\left(s-M_{\Phi}^{2}\right)}{2 M_{\Phi}}
$$

e $\lambda_{0} \simeq\left(M_{h}+\varepsilon\right)$, onde $M_{h}$ é a massa do projétil e

$$
\varepsilon=2 m_{M}-M_{\Phi} .
$$

Na figura 6.2, mostramos as seções de choque de dissociação próton-charmonium obtida com o modelo $A$ (linha pontilhada) e com o modelo $B$ (linha sólida com estrelas) dada por $(6,3.28)$. As duas curvas superiores foram obtidas com $\langle h|g E| h\rangle=1 \mathrm{GeV} / \mathrm{fm}$ e a duas inferiores com $\langle h|g E| h\rangle=0.57 \mathrm{GeV} / \mathrm{fm}$ (modelo $A$ ) and $\langle h|g E| h\rangle=0.53$ $\mathrm{GeV} / \mathrm{fm}$ (modelo $B$ ). Com valores menores do campo cromoelétrico nossas curvas ficam próximas as da referência [85]. A figura 6.3, mostra as seções de choque de dissociação para próton-bottonium. Novamente as curvas superiores são para $\langle h|g E| h\rangle=1 \mathrm{GeV} / \mathrm{fm}$ e a duas inferiores para $\langle h|g E| h\rangle=0.69 \mathrm{GeV} / \mathrm{fm}$ (modelo A) and $\langle h|g E| h\rangle=0.49 \mathrm{GeV} / \mathrm{fm}$ (modelo B). Como na figura anterior, reduzindo-se o valor de $\langle h|g E| h\rangle$ leva-nos a um acordo com (6,3.28). Dada a semelhança conceitual entre nosso modelo e o de BP, é uma reafirmação encontrar uma certa similaridade entre os resultados, ambos em magnitude e no comportamento com a energia, uma vez que um valor apropriado de $\langle h|g E| h\rangle$ é escolhido.

Na figura 6.4, mostramos a seção de choque para $J / \psi$ de dissociação por píons comparada com resultados obtidos com o modelo de troca de mésons [81] (linha pontilhada fina), com o modelo de troca de quarks [80] (linha tracejada longa fina), com 


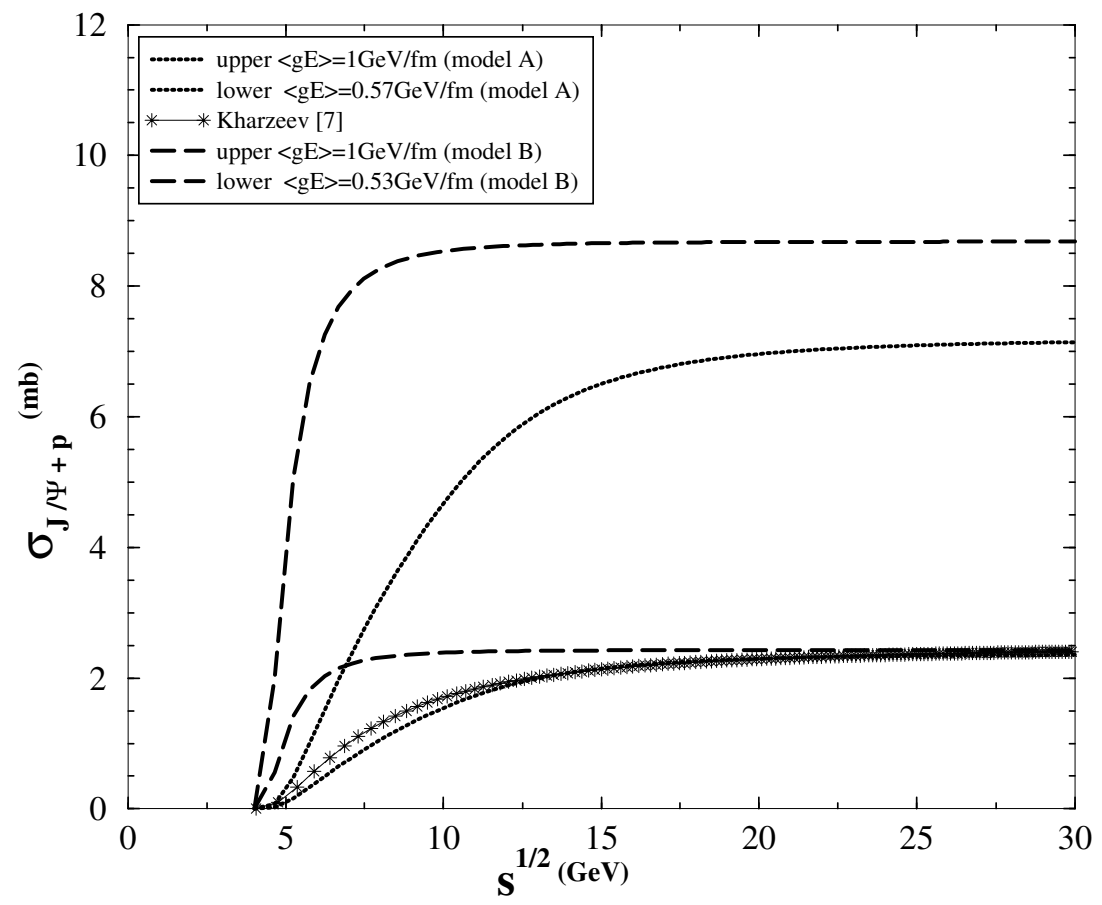

Figura 6.2: Seção de choque $J / \psi+p$ com o modelo $A$ (linha pontilhada), modelo $B$ (linha tracejada) e a aproximação de Bhanot-Peskin (linha com estrelas). Curvas superiores: Campo $\vec{E}^{a}$ mais forte. Curvas inferiores: Campo $\vec{E}^{a}$ mais fraco.

o modelo de curtas distâncias (aproximação de BP) equação $(6,3.28)$ (linha sólida espessa) com regras de soma da QCD [79] (linha sólida fina). Apesar do fato de nossa aproximação perder a validade em baixas energias, é interessante observar que nossa curva está numa região central coberta por outros cálculos. Na figura 6.5, comparamos as seções de choque $p+J / \psi$ (curvas superiores) and $\pi+J / \psi$ (curvas inferiores) calculadas com os modelos $A$ (linhas pontilhadas) e $B$ (linhas tracejadas). No limite de altas energias, onde ambas as seções de choque são quase constantes, observamos que a relação entre as seções de choque é:

$$
\begin{gathered}
\sigma_{p+\Phi} \simeq 3 \sigma_{\pi+\Phi} \text { model } \mathrm{A} \\
\sigma_{p+\Phi} \simeq 4.2 \sigma_{\pi+\Phi} \quad \text { model } \mathrm{B}
\end{gathered}
$$




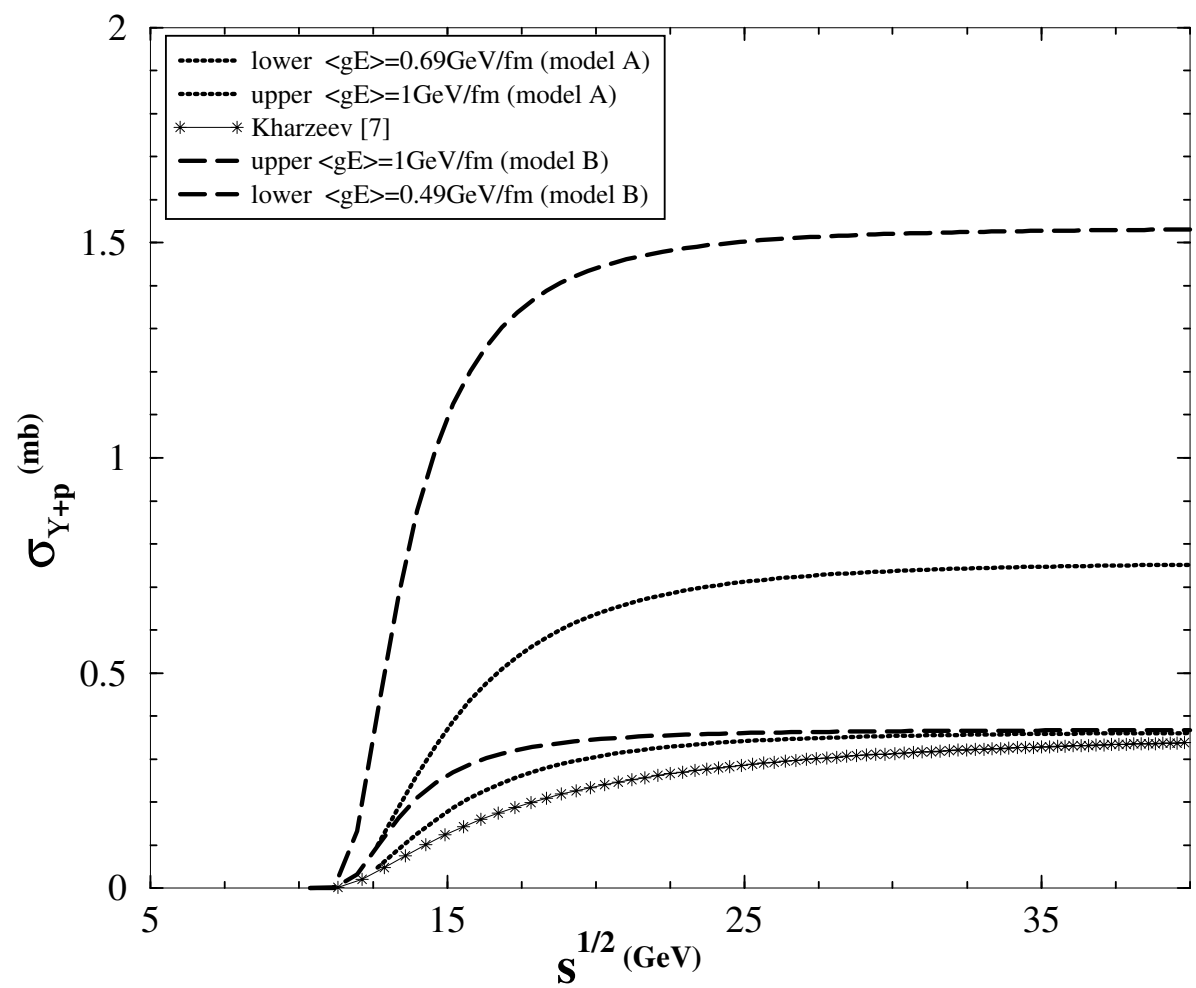

Figura 6.3: $O$ mesmo como na figura 1.2 para a seção de choque $\Upsilon+p$.

as quais são nos dois casos muito maiores do que aquela esperada do modelo aditivo de quarks:

$$
\sigma_{p+\Phi} \simeq \frac{3}{2} \sigma_{\pi+\Phi}
$$

Isso é interessante uma vez que a relação do modelo aditivo de quarks vale para outros espalhamento de altas energias como $\pi+p$ e $p+p$. Como $\langle h|g E| h\rangle$ foi mantido o mesmo nos dois casos, essa relação, não esperada entre as seções de choque, deve vir das diferenças entre as funções de onda. Na figura 6.6, repetimos essa comparação para as reações $p+\Upsilon$ e $\pi+\Upsilon$, encontrando $(6,3.31)$ para ambos os modelos. Mantenos $\langle h|g E| h\rangle=1 \mathrm{GeV} / \mathrm{fm}$ para ambos os projéteis. Se $\langle p|g E| p\rangle>\langle\pi|g E| \pi\rangle$ o desvio de $(6,3.33)$ é ainda maior.

No limite de altas energias os hádrons ordinários devem ter uma seção de choque 


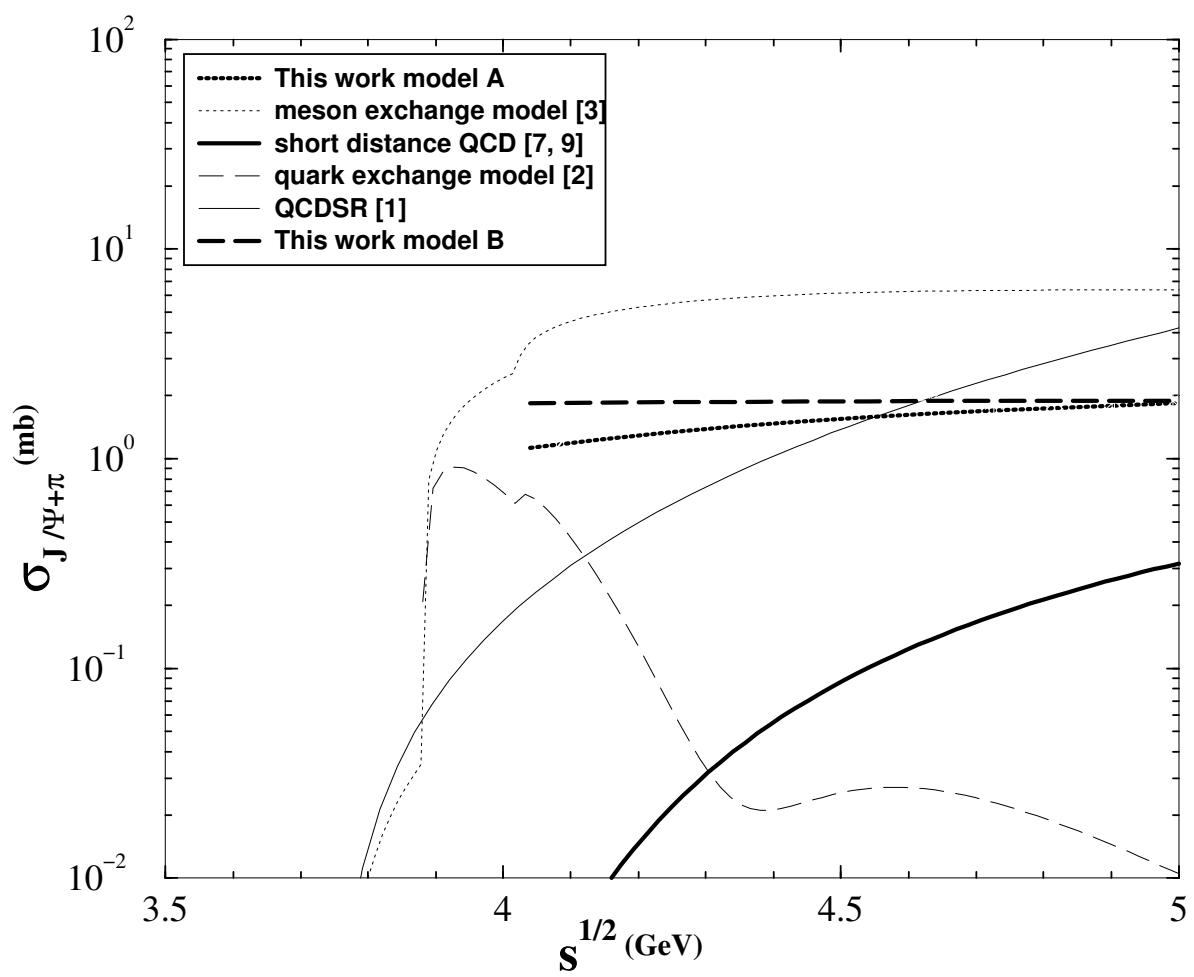

Figura 6.4: Seção de choque Píon-charmonium como uma função de $\sqrt{s}$ com vários modelos.

total geométrica. Como a dissociação do quarkonium discutida é uma reação mais específica, não é óbvio que sua seção de choque tenha um comportamento geométrico. Em [86] foi encontrado o seguinte comportamento $\sigma_{\Phi h} \propto \alpha_{s} a_{0}^{2}$ onde $a_{0}$ é o raio de Bohr do quarkonium. Em nosso caso, como pode ser visto de $(6,2.16),(6,2.27)$ e do cálculo numérico de $(6,2.14)$, temos uma dependência não trivial em $a$. Desde que o estado inicial (contendo a variável a) é o mesmo, a diferença entre os modelos vêm da dependência espacial do estado final. As ondas planas no modelo $A$ não têm escala espacial. Entretanto elas são mais inclusivos e assim $\sigma_{A}$ estaria mais próxima da seção de choque total quarkonium-hádron do que $\sigma_{B}$. No modelo $B$ o estado fundamental do quarkonium é convertido em um estado tipo ressonância, a função de onda deste contém o parâmetro de tamanho da ressonância e distorce o comportamento geométrico final. Portanto, o modelo $A$ tem um comportamento mais geométrico do que o modelo $B$.

Para visualizarmos o quão longe estamos do comportamento geométrico, mostra- 


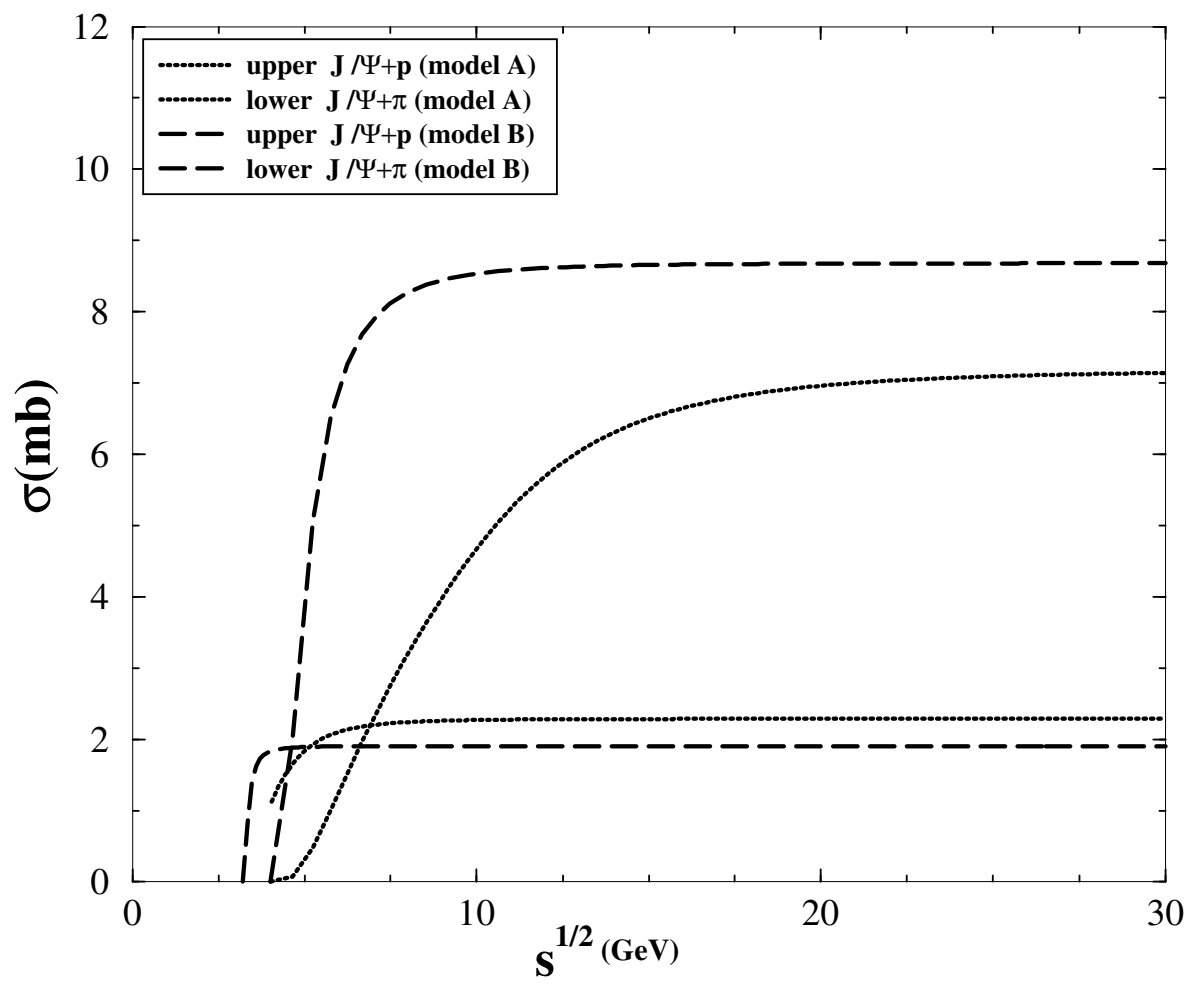

Figura 6.5: Seção de choque charmonium-hádron com o modelo $A$ (linha pontilhada) e com o modelo $B$ (linha tracejada). Curvas superiores: $\sigma_{J / \psi}+p$. Curvas inferiores $\sigma_{J / \psi}+\pi$.

mos nas figuras 6.7 e 6.8, a dependência de $\sigma_{A}$ (linha pontilhada) e $\sigma_{B}$ (linha tracejada) em função de $a$ para a dissociação do charmonium (figura 6.7) e do bottonium (figura 6.8). As seções de choque foram divididas por $a^{2}$ de forma que o comportamento geométrico seja representado por uma linha horizontal. Vemos que, enquanto o modelo $A$ tende a esse comportamento, o modelo $B$ está longe do comportamento geométrico. Isso indica novamente que nosso modelo é muito sensível à escolha das funções de onda do estado final.

Na figura 6.9, mostramos a seção de choque de dissociação $\sigma_{C}(6,2.27)$, por prótons (linha sólida) e por píons (linha tracejada). Na figura 6.10, mostramos a mesma quantidade para a dissociação do bottonium. Usamos os valores centrais para $a, a^{\prime}$, $d$ e $\alpha_{s}$. Vemos que em todos os processos, as seções de choque, são mais do que duas ordens de magnitude menor do que as seções de choque correspondentes ao modelo $A$ ou ao modelo $B$. Nenhuma mudança nos parâmetros poderia tornar essas seções de 


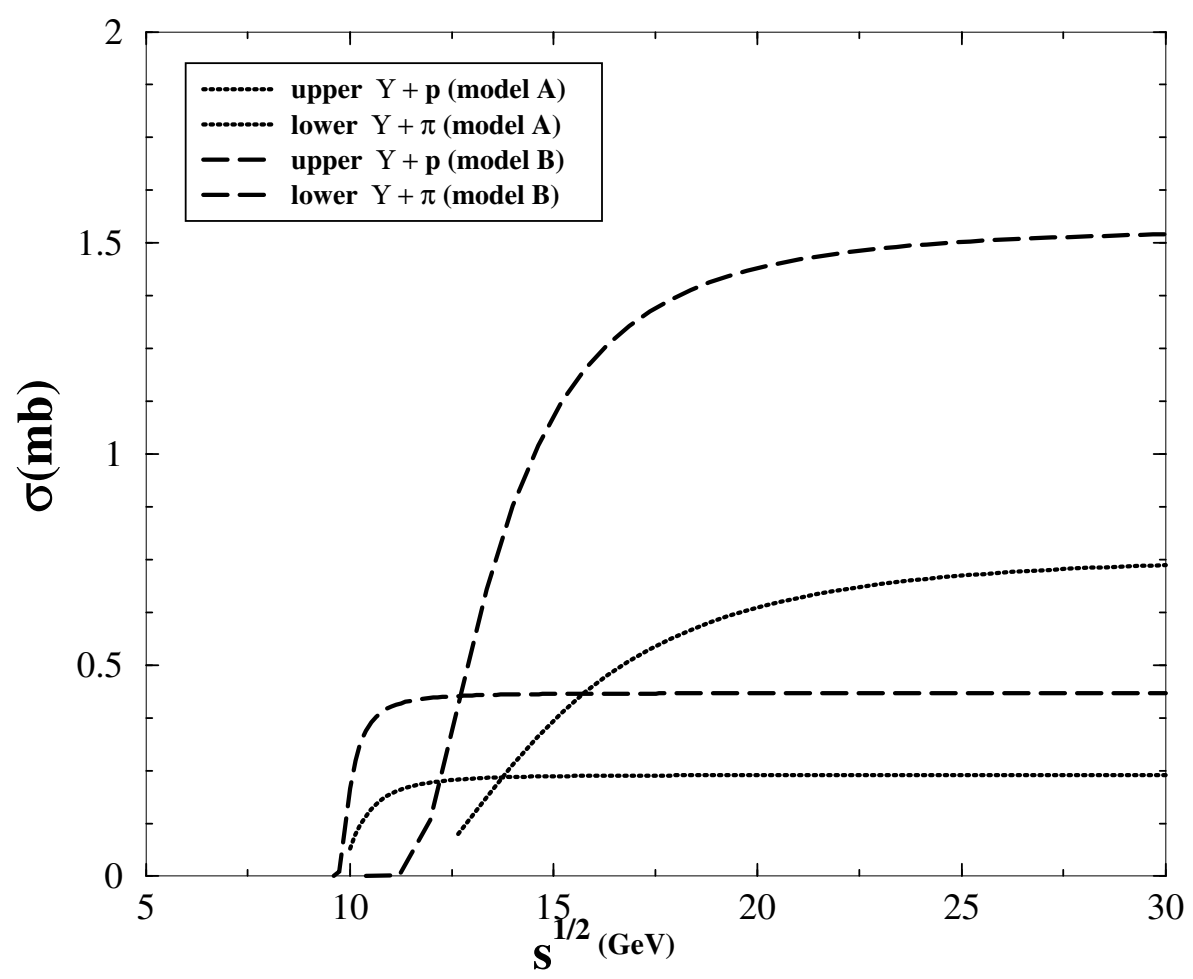

Figura 6.6: $O$ mesmo como na figura 1.5, para a seção de choque bottonium-hádron.

choque comparáveis. Outra característica dessas curvas é que as seções de choque para a dissociação de $J / \psi$ por píons são maiores do que aquelas para prótons por um fator próximo de 4. Isso é bem razoável quando observamos $(6,2.27)$. O píon é um sistema leve de quark-antiquark e o próton é um sistema leve de quark-diquark. O diquark é duas vezes mais pesado do que o quark constituinte. Para o píon temos $m_{b}=m_{a}$, e para o próton $m_{b}=2 m_{a}$.

Na figura 6.11, vemos que a probabilidade máxima de interação, ou seja, a probabilidade do par encontrar uma das placas do capacitor, ocorre para um tamanho do $\operatorname{par} a \approx 0.5 \mathrm{fm}$.

Na figura 6.12, vemos que quando o tamanho do capacitor aumenta (o parâmetro $d$ aumenta), a probabilidade de um dos quarks do charmonium (e/ou bottonium) encontrar uma das placas do capacitor, diminui.

Nas figuras 6.13 e 6.14 , mostramos a seção de choque de dissociação do $J / \Psi$ por prótons e píons, para vários valores de $a$ e $d$ fixados, respectivamente. Vemos que 


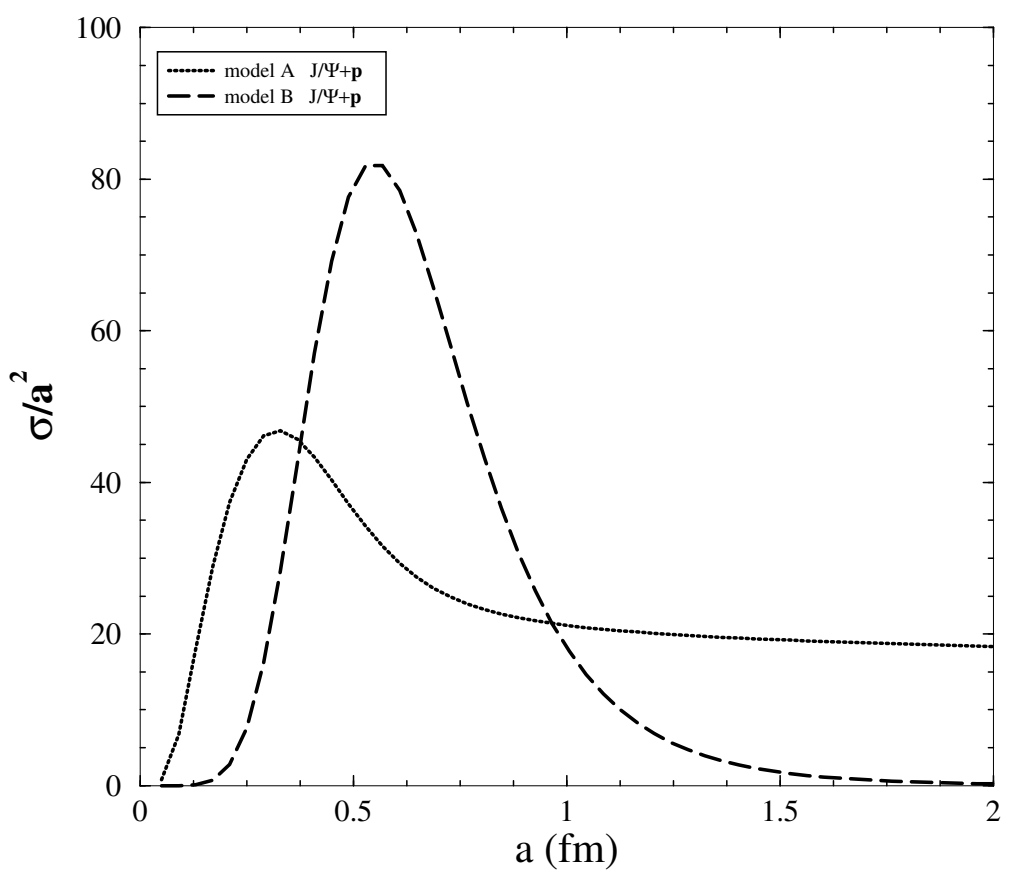

Figura 6.7: Seção de choque charmonium-hádron como uma função do parâmentro de tamanho do charmonium para o modelo $A$ (linha pontilhada) e para o modelo $B$ (linha tracejada).

quando aumentamos o valor de $a$ a seção de choque é maior (figura 6.13), e quando aumentamos $d$ esta torna-se menor (figura 6.14), como esperado. 


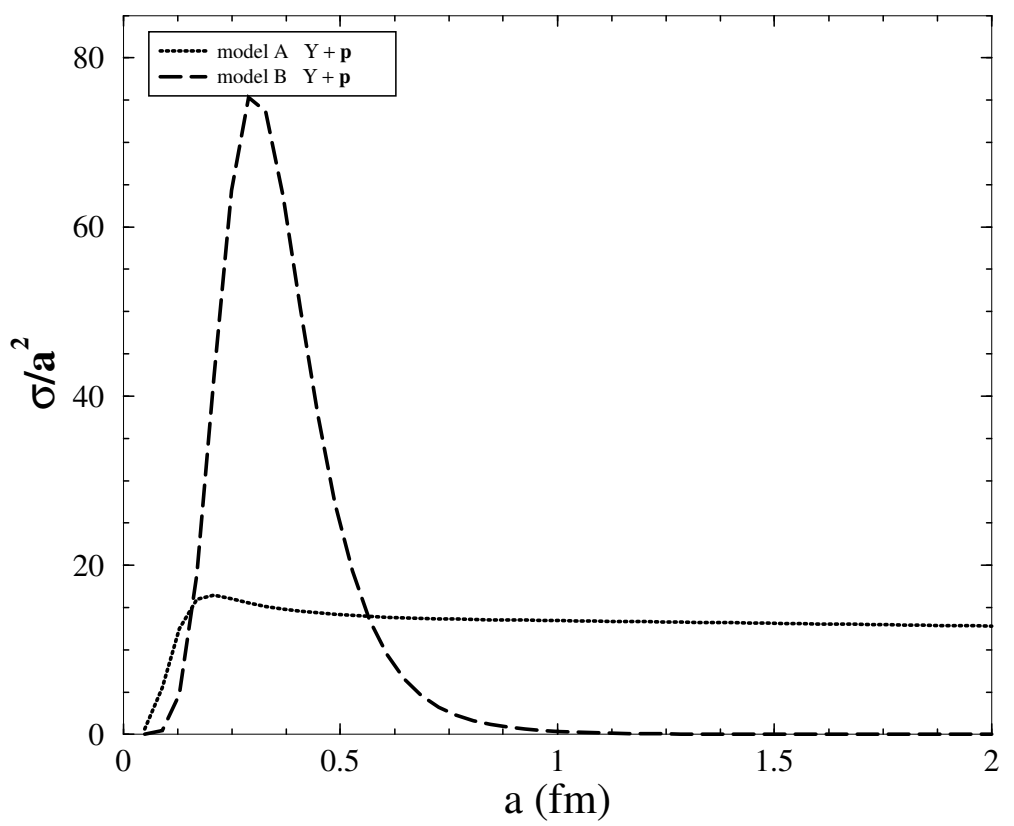

Figura 6.8: $O$ mesmo como na figura 6.7, para a seção de choque bottonium-hádron.

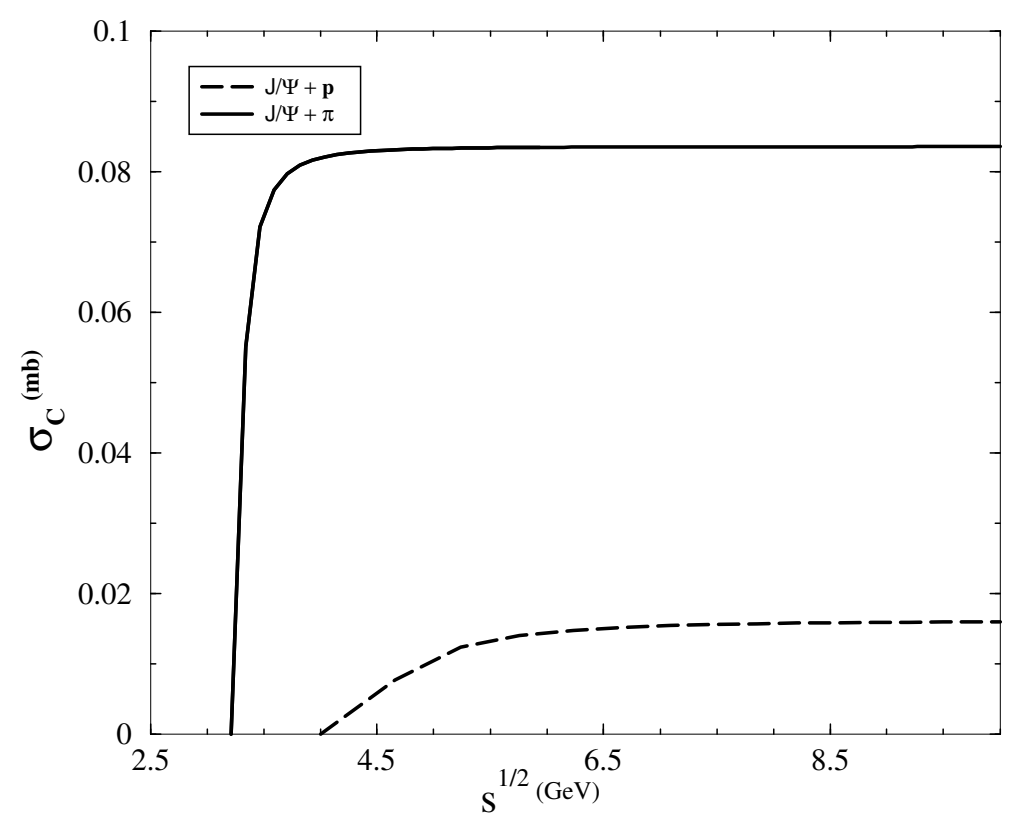

Figura 6.9: Seção de choque charmonium-próton (linha tracejada) e charmonium-píon (linha sólida) calculada com o modelo $C$ (interação de contato). 


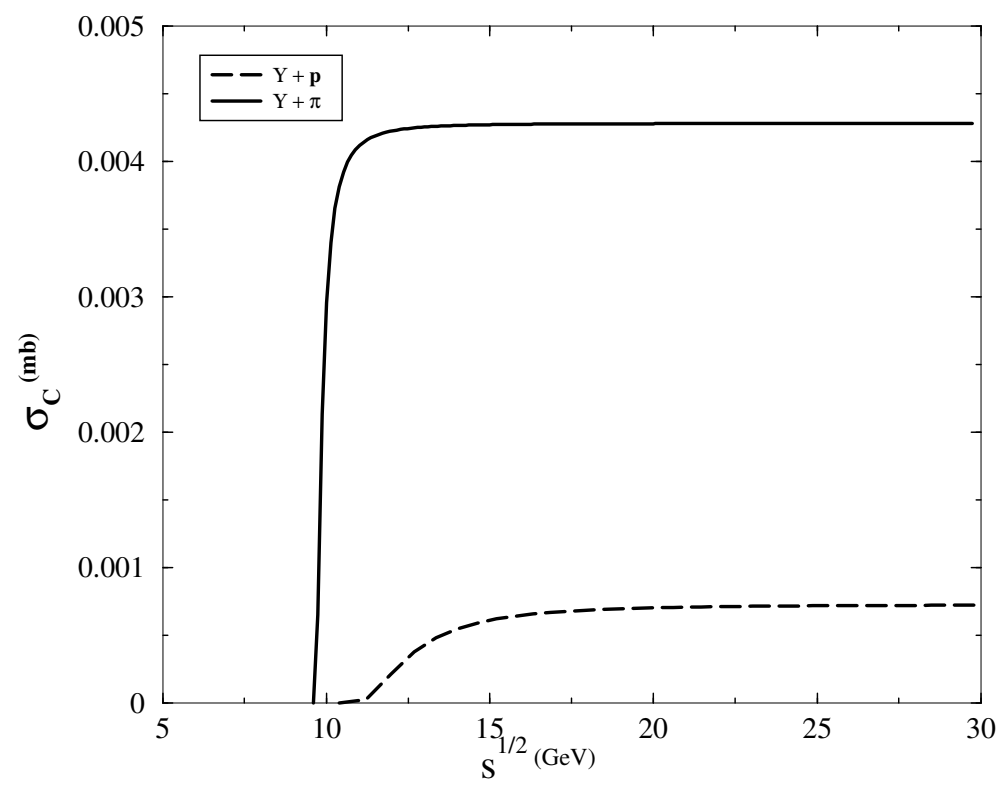

Figura 6.10: $O$ mesmo como na figura 6.9, para a seção de choque bottonium-hádron.

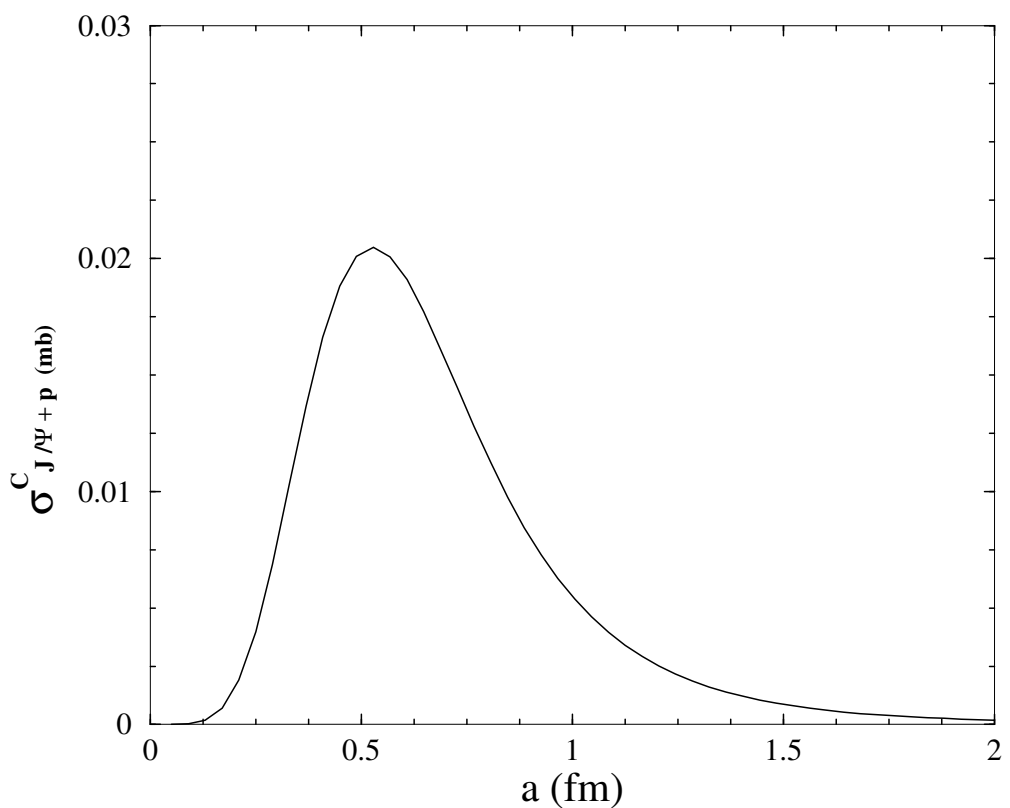

Figura 6.11: Seção de choque charmonium-hádron para o modelo C, como uma função do parâmetro $a$. 


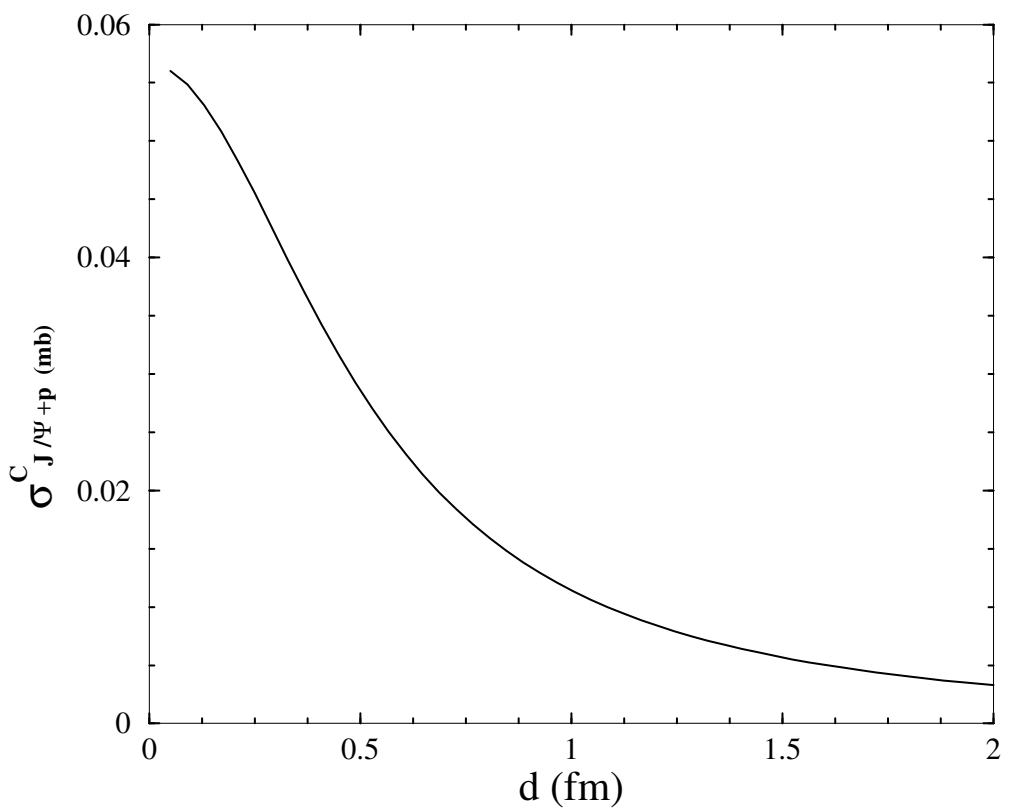

Figura 6.12: Seção de choque charmonium-hádron para o modelo C, como uma função do parâmetro $d$.

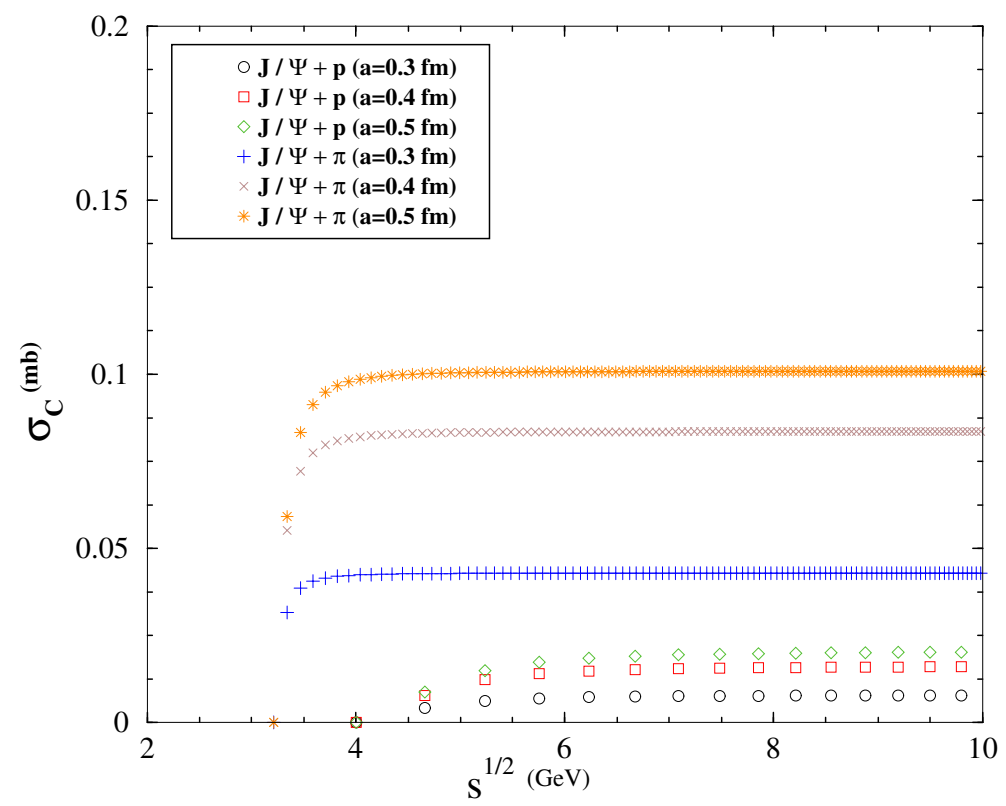

Figura 6.13: Seção de choque charmonium-hádron para o modelo $C$, como uma função de $\sqrt{s}$, para vários valores fixos do parâmetro $a$. 


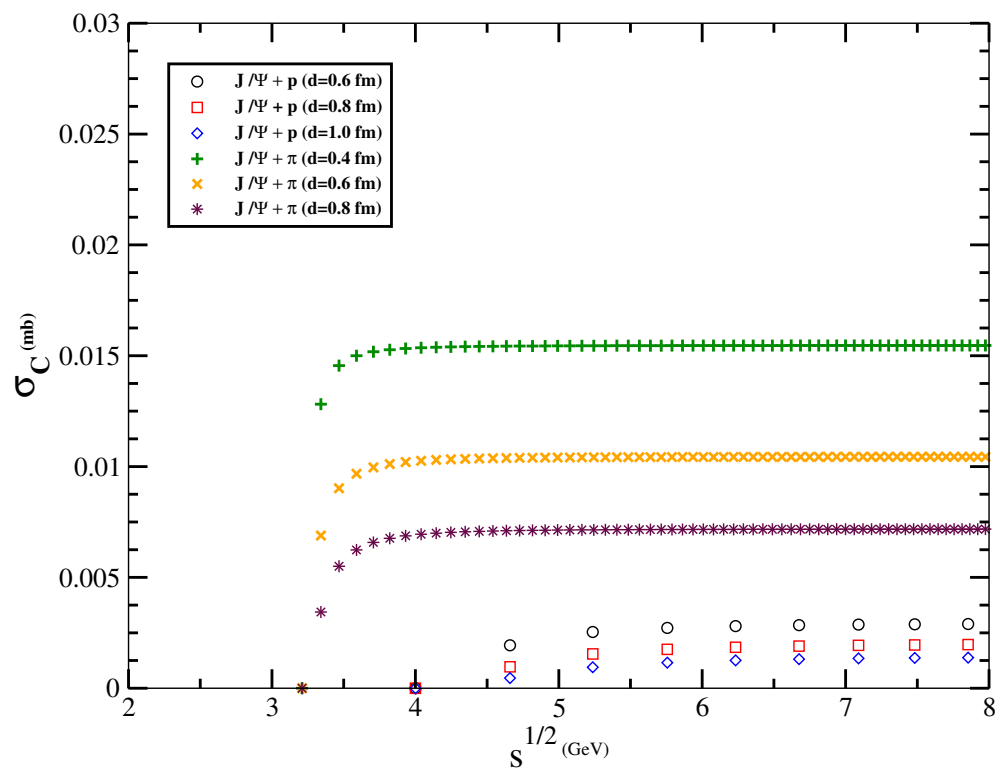

Figura 6.14: Seção de choque charmonium-hádron para o modelo $\boldsymbol{C}$, como uma função de $\sqrt{s}$, para vários valores fixos do parâmetro $d$. 


\section{Capítulo 7}

\section{Conclusão}

Apesar de ser um trabalho de pesquisa esta tese tinha também como objetivo ser um texto pedagógico, destinado aos interessados em estudar física da saturação. Assim, nos capítulos 1, 2 e 3 fizemos um esforço no sentido de organizar de maneira didática o material disponível na literatura.

No capítulo 4 comparamos os vários modelos existentes para a seção de choque de dipolo entre si e com os dados experimentais, fazendo um cruzamento das informações experimentais vindas do espalhamento inelástico profundo (as medidas feitas no HERA) com os resultados experimentais obtidos em colisões de íons pesados no RHIC. A conclusão, um pouco surpreendente, foi que as parametrizações que funcionavam bem num contexto não funcionavam no outro. Em vista disto apresentamos o nosso próprio Ansatz, que descreve bem os dados de uma maneira global. A forma da seção de choque de dipolo tem que satisfazer várias condições e reproduzir resultados obtidos mais rigorosamente em certos limites. Isto limita as mudanças que podem ser feitas nas parametrizações existentes. Na prática, em vários trabalhos o que é feito é alterar a dimensão anômala efetiva. Foi isto o que fizemos também, de uma maneira bem fenomenológica.

No capítulo 5 fizemos previsões para o comportamento de vários observáveis a serem medidos num acelerador elétron-íon, que talvez seja construído onde hoje é o RHIC. A decisão de construir ou não o acelerador será tomada em breve e terá que 
levar em conta o potencial desta máquina para observar as propriedades do CGC. Nós estudamos como a mudança do alvo, de um próton para um núcleo pesado pode alterar as funções de estrutura inclusiva e difrativa e a razão das seções de choque difrativa e inclusiva. Particularmente relevantes são os resultados da figura 5.7, que mostram que, para as razões $R_{F_{2}}$ e $R_{F_{L}}$, a diferença entre a física linear e a total pode alterar os resultados por um fator de até 2! O comportamento das razões entre funções de estrutura difrativas como função de $x_{\mathbb{P}}$ e $\beta$ também mostra que os efeitos da física não-linear fazem com que estas razões sejam muito fracamente dependentes destas variáveis, produzindo uma espécie de "scaling", que é visível quando usamos núcleos pesados. Finalmente o crescimento da razão entre $\sigma_{d i f f}$ e $\sigma_{t o t}$ é bastante significativo.

Nossos resultados foram obtidos com uma única seção de choque de dipolo (IIM) e com uma maneira um pouco simplista de introduzir a dependência nuclear. Apesar disto, como primeiras estimativas eles são bastante promissores. Eles nos encorajam a, no futuro, fazer um estudo mais sistemático. Nossas previsões passarão a ter um "erro teórico" que ainda não foi estimado. 


\section{Apêndice}

\section{A Equação de BFKL}

A equação proposta por Balitsky, Fadin, Kuraev e Lipatov (BFKL) ressoma termos do tipo $\alpha_{s} \ln (1 / x)$ e descreve a evolução da distribuição de gluons não integrada $f\left(x, k^{2}\right)$, a qual nos fornece a probabilidade de encontrar um glúon no hádron com fração de momento longitudinal $x$ e momento transversal $k$. Esta relaciona-se com $x g\left(x, Q^{2}\right)$ por $(2,1.3)$ :

$$
g\left(x, Q^{2}\right)=\int^{Q^{2}} \frac{d \vec{K}^{2}}{\vec{K}^{2}} f\left(x, \vec{K}^{2}\right)
$$

A distribuição de gluons não integrada $f\left(x, k^{2}\right)$, satisfaz a seguinte equação:

$$
\begin{aligned}
-x \frac{\partial f\left(x, k^{2}\right)}{\partial x} & =\frac{3 \alpha_{s}}{\pi} k^{2} \int_{0}^{\infty} \frac{d k^{\prime 2}}{k^{\prime 2}}\left[\frac{f\left(x, k^{\prime 2}\right)-f\left(x, k^{2}\right)}{\left|k^{\prime 2}-k^{2}\right|}+\frac{f\left(x, k^{2}\right)}{\left(4 k^{\prime 4}+k^{4}\right)^{\frac{1}{2}}}\right] \\
& \equiv \mathcal{K} \otimes f .
\end{aligned}
$$

Considerando a transformada de Mellin:

$$
\tilde{f}(x, \gamma)=\int_{0}^{\infty}\left(k^{2}\right)^{-\gamma-1} f\left(x, k^{2}\right) d k^{2}
$$

e a sua inversa

$$
f\left(x, k^{2}\right)=\frac{1}{2 \pi i} \int_{c-i \infty}^{c+i \infty}\left(k^{2}\right)^{\gamma} \tilde{f}(x, \gamma) d \gamma
$$


teremos que

$$
\begin{gathered}
\frac{1}{2 \pi i} \int_{c-i \infty}^{c+i \infty}\left(k^{2}\right)^{\gamma}\left[-x \frac{\partial \tilde{f}\left(x, k^{2}\right)}{\partial x}\right] d \gamma=\frac{3 \alpha_{s}}{\pi} k^{2} \frac{1}{2 \pi i} \int_{c-i \infty}^{c+i \infty} d \gamma \int_{0}^{\infty} \frac{d k^{\prime 2}}{k^{\prime 2}} \\
\times\left[\frac{\left(k^{\prime 2}\right)^{\gamma} \tilde{f}(x, \gamma)-\left(k^{2}\right)^{\gamma} \tilde{f}(x, \gamma)}{\left|k^{\prime 2}-k^{2}\right|}+\frac{\left(k^{2}\right)^{\gamma} \tilde{f}(x, \gamma)}{\left(4 k^{\prime}+k^{4}\right)^{\frac{1}{2}}}\right]
\end{gathered}
$$

Fazendo a mudança de variável: $k^{\prime 2}=k^{2} \mu$, teremos:

$$
\begin{array}{r}
\frac{1}{2 \pi i} \int_{c-i \infty}^{c+i \infty}\left(k^{2}\right)^{\gamma}\left[-x \frac{\partial \tilde{f}\left(x, k^{2}\right)}{\partial x}\right] d \gamma=\frac{3 \alpha_{s}}{\pi} k^{2} \frac{1}{2 \pi i} \int_{c-i \infty}^{c+i \infty} d \gamma \int_{0}^{\infty} \frac{d \mu}{\mu} \\
\times\left[\frac{\left(\mu k^{2}\right)^{\gamma} \tilde{f}(x, \gamma)-\left(k^{2}\right)^{\gamma} \tilde{f}(x, \gamma)}{\left|\mu k^{2}-k^{2}\right|}+\frac{\left(k^{2}\right)^{\gamma} \tilde{f}(x, \gamma)}{\left(4 \mu^{2} k^{4}+k^{4}\right)^{\frac{1}{2}}}\right] \\
\frac{1}{2 \pi i} \int_{c-i \infty}^{c+i \infty}\left(k^{2}\right)^{\gamma}\left[-x \frac{\partial \tilde{f}\left(x, k^{2}\right)}{\partial x}\right] d \gamma=\left(k^{2}\right)^{\gamma} \frac{1}{2 \pi i} \int_{c-i \infty}^{c+i \infty} d \gamma \frac{3 \alpha_{s}}{\pi} \int_{0}^{\infty} \frac{d \mu}{\mu} \\
\times\left[\frac{1}{|\mu-1|}\left(\mu^{\gamma}-1\right) \tilde{f}(x, \gamma)+\frac{\tilde{f}(x, \gamma)}{\left(4 \mu^{2}+1\right)^{\frac{1}{2}}}\right]
\end{array}
$$

onde identificamos:

$$
-x \frac{\partial \tilde{f}(x, \gamma)}{\partial x}=\frac{3 \alpha_{s}}{\pi} \int_{0}^{\infty} \frac{d \mu}{\mu}\left[\frac{\mu^{\gamma}-1}{|\mu-1|}+\frac{1}{\left(4 \mu^{2}+1\right)^{\frac{1}{2}}}\right] \tilde{f}(x, \gamma)
$$

Aqui definimos o kernel da BFKL como:

$$
\begin{aligned}
\tilde{k}(\gamma) & \equiv \frac{3 \alpha_{s}}{\pi} \int_{0}^{\infty} \frac{d \mu}{\mu}\left[\frac{\mu^{\gamma}-1}{|\mu-1|}+\frac{1}{\left(4 \mu^{2}+1\right)^{\frac{1}{2}}}\right] \\
& =\frac{3 \alpha_{s}}{\pi}[2 \Psi(1)-\Psi(\gamma)-\Psi(1-\gamma)]
\end{aligned}
$$

onde $\Psi(\gamma)$ é a função Digamma. De $(0,0.5)$ obtemos :

$$
-x \frac{\partial \tilde{f}(x, \gamma)}{\partial x}=\tilde{k}(\gamma) \tilde{f}(x, \gamma)
$$


Resolvendo a equação acima temos que:

$$
\tilde{f}(x, \gamma)=\tilde{f}\left(x_{0}, \gamma\right)\left(\frac{x}{x_{0}}\right)^{-\tilde{k}(\gamma)}
$$

Inserindo $(0,0.8)$ em $(0,0.4)$ :

$$
f\left(x, k^{2}\right)=\frac{1}{2 \pi i} \int_{c-i \infty}^{c+i \infty}\left(k^{2}\right)^{\gamma} d \gamma \tilde{f}\left(x_{0}, \gamma\right)\left(\frac{x}{x_{0}}\right)^{-\tilde{k}(\gamma)}
$$

Tomando $\gamma=\frac{1}{2}+i \nu$ e expandindo $\tilde{k}(\gamma)$ e $\tilde{f}(x, \gamma)$ em torno de $\gamma=\frac{1}{2}$. (Note que quando plotamos o gráfico de $\tilde{k}(\gamma)$ em função de $\gamma$, vemos que o ponto de mínimo ocorre para $\gamma=\frac{1}{2}$.)

$$
\begin{aligned}
\tilde{k}\left(\frac{1}{2}+i \nu\right) & =\tilde{k}\left(\frac{1}{2}\right)+\left.\frac{\partial \tilde{k}}{\partial \gamma}\right|_{\gamma=\frac{1}{2}}\left(\gamma-\frac{1}{2}\right)+\left.\frac{1}{2} \frac{\partial^{2} \tilde{k}}{\partial^{2} \gamma}\right|_{\gamma=\frac{1}{2}}\left(\gamma-\frac{1}{2}\right)^{2}+\ldots \\
& =\tilde{k}\left(\frac{1}{2}\right)+\left.\frac{\partial \tilde{k}}{\partial \gamma}\right|_{\gamma=\frac{1}{2}}(i \nu)+\left.\frac{1}{2} \frac{\partial^{2} \tilde{k}}{\partial^{2} \gamma}\right|_{\gamma=\frac{1}{2}}(i \nu)^{2}+\ldots
\end{aligned}
$$

mas $\frac{\partial \tilde{k}}{\partial \gamma}=0$ no ponto de mínimo. Além disso:

$$
\begin{aligned}
\tilde{k}\left(\frac{1}{2}\right) & =\frac{3 \alpha_{s}}{\pi}\left[2 \Psi(1)-\Psi\left(\frac{1}{2}\right)-\Psi\left(1-\frac{1}{2}\right)\right] \\
& =\frac{6 \alpha_{s}}{\pi}\left[\Psi(1)-\Psi\left(\frac{1}{2}\right)\right] \\
& =\frac{6 \alpha_{s}}{\pi}[-\gamma-(-\gamma-2 \ln 2)] \\
& =\frac{3 \alpha_{s}}{\pi} 4 \ln 2
\end{aligned}
$$

e

$$
\begin{aligned}
\left.\frac{\partial^{2} \tilde{k}}{\partial^{2} \gamma}\right|_{\gamma=\frac{1}{2}} & =\left.\frac{\partial^{2}}{\partial^{2} \gamma}\left\{\frac{3 \alpha_{s}}{\pi}[2 \Psi(1)-\Psi(\gamma)-\Psi(1-\gamma)]\right\}\right|_{\gamma=\frac{1}{2}} \\
& =-\left.\frac{3 \alpha_{s}}{\pi}\left[\Psi^{\prime \prime}(\gamma)+\Psi^{\prime \prime}(1-\gamma)\right]\right|_{\gamma=\frac{1}{2}}
\end{aligned}
$$


Como

$$
\begin{aligned}
\Psi^{\prime \prime}(1-z)-\Psi^{\prime \prime}(z) & =\pi \frac{d^{2}}{d z^{2}} \operatorname{cotg} \pi z \\
& =-\left.2 \pi^{3} \csc ^{2} \pi z \operatorname{cotg} \pi z\right|_{z=\frac{1}{2}} \\
& =0
\end{aligned}
$$

então $\Psi^{\prime \prime}(1-z)=\Psi^{\prime \prime}(z)$ e obtemos

$$
\begin{aligned}
\left.\frac{\partial^{2} \tilde{k}}{\partial^{2} \gamma}\right|_{\gamma=\frac{1}{2}} & =-\left.\frac{3 \alpha_{s}}{\pi} 2 \Psi^{\prime \prime}(\gamma)\right|_{\gamma=\frac{1}{2}} \\
& =-\frac{3 \alpha_{s}}{\pi} 2 \Psi^{\prime \prime}\left(\frac{1}{2}\right) \\
& =-\frac{3 \alpha_{s}}{\pi} 2(-1) 2 !\left(2^{3}-1\right) \zeta(3) \\
& =\frac{3 \alpha_{s}}{\pi} 28 \zeta(3)
\end{aligned}
$$

Assim a expansão em $\tilde{k}$ resulta:

$$
\begin{aligned}
\tilde{k}\left(\frac{1}{2}+i \nu\right) & =\tilde{k}\left(\frac{1}{2}\right)+\left.\frac{1}{2} \frac{\partial^{2} \tilde{k}}{\partial^{2} \gamma}\right|_{\gamma=\frac{1}{2}}(i \nu)^{2}+\ldots \\
& =\frac{3 \alpha_{s}}{\pi} 4 \ln 2-\frac{1}{2} \frac{3 \alpha_{s}}{\pi} 28 \zeta(3) \nu^{2}+\mathcal{O}\left(\nu^{4}\right) \\
& =\lambda-\frac{1}{2} \lambda^{\prime \prime} \nu^{2}+\mathcal{O}\left(\nu^{4}\right)
\end{aligned}
$$

onde identificamos

$$
\begin{gathered}
\lambda=\frac{3 \alpha_{s}}{\pi} 4 \ln 2 \\
\lambda^{\prime \prime}=\frac{3 \alpha_{s}}{\pi} 28 \zeta(3)
\end{gathered}
$$

$\operatorname{com} \zeta(3)=1,202$ 
Expandindo $\tilde{f}\left(x_{0}, \gamma\right)$ em torno de $\gamma=\frac{1}{2}$ (que é mesmo que $\nu=0$ ).

$$
\begin{aligned}
\tilde{f}\left(x_{0}, \gamma\right) & =\left.\tilde{f}\left(x_{0}, \gamma\right)\right|_{\nu=0}+\left.\frac{\partial \tilde{f}}{\partial \gamma}\right|_{\nu=0}\left(\gamma-\frac{1}{2}\right)+\left.\frac{1}{2} \frac{\partial^{2} \tilde{f}}{\partial^{2} \gamma}\right|_{\nu=0}\left(\gamma-\frac{1}{2}\right)^{2}+\ldots \\
& =\tilde{f}\left(x_{0}, \frac{1}{2}\right)+\left.\frac{\partial \tilde{f}}{\partial \gamma}\right|_{\nu=0}(i \nu)+\left.\frac{1}{2} \frac{\partial^{2} \tilde{f}}{\partial^{2} \gamma}\right|_{\nu=0}(i \nu)^{2}+\ldots
\end{aligned}
$$

Inserindo $(0,0.12)$ e $(0,0.15)$ em $(0,0.9)$ :

$$
\begin{aligned}
f\left(x, k^{2}\right)= & \frac{1}{2 \pi} \int_{-\infty}^{+\infty} d \nu\left(k^{2}\right)^{\frac{1}{2}+i \nu}\left(\frac{x}{x_{0}}\right)^{-\lambda+\frac{1}{2} \lambda^{\prime \prime} \nu^{2}} \\
& \times \tilde{f}\left(x_{0}, \frac{1}{2}\right)\left\{1+\left.\frac{i \nu}{\tilde{f}} \frac{\partial \tilde{f}}{\partial \gamma}\right|_{\nu=0}-\left.\frac{\nu^{2}}{\tilde{f}} \frac{1}{2} \frac{\partial^{2} \tilde{f}}{\partial^{2} \gamma}\right|_{\nu=0}+\ldots\right\} \\
= & \frac{1}{2 \pi}\left(k^{2}\right)^{\frac{1}{2}} \tilde{f}\left(x_{0}, \frac{1}{2}\right)\left(\frac{x}{x_{0}}\right)^{-\lambda} \int_{-\infty}^{+\infty} d \nu\left(\frac{x}{x_{0}}\right)^{\frac{1}{2} \lambda^{\prime \prime} \nu^{2}}\left(k^{2}\right)^{i \nu} \\
& \times\left\{1+\left.\frac{i \nu}{\tilde{f}} \frac{\partial \tilde{f}}{\partial \gamma}\right|_{\nu=0}-\left.\frac{\nu^{2}}{\tilde{f}} \frac{1}{2} \frac{\partial^{2} \tilde{f}}{\partial^{2} \gamma}\right|_{\nu=0}+\ldots\right\}
\end{aligned}
$$

onde identificamos:

$$
1+\left.\frac{i \nu}{\tilde{f}} \frac{\partial \tilde{f}}{\partial \gamma}\right|_{\nu=0}-\left.\frac{\nu^{2}}{\tilde{f}} \frac{1}{2} \frac{\partial^{2} \tilde{f}}{\partial^{2} \gamma}\right|_{\nu=0}+\ldots=\exp \left\{\left.i \nu \frac{\partial \ln \tilde{f}}{\partial \gamma}\right|_{\nu=0}\right\}
$$

Podemos escrever:

$$
\left(k^{2}\right)^{i \nu}=\exp \left\{i \nu \ln k^{2}\right\}
$$

e

$$
\left(\frac{x}{x_{0}}\right)^{\frac{1}{2} \lambda^{\prime \prime} \nu^{2}}=\exp \left\{\frac{1}{2} \lambda^{\prime \prime} \ln \left(\frac{x}{x_{0}}\right) \nu^{2}\right\}
$$

Então:

$$
\begin{aligned}
f\left(x, k^{2}\right) & =\frac{1}{2 \pi}\left(k^{2}\right)^{\frac{1}{2}} \tilde{f}\left(x_{0}, \frac{1}{2}\right)\left(\frac{x}{x_{0}}\right)^{-\lambda} \int_{-\infty}^{+\infty} d \nu \exp \left\{\frac{1}{2} \lambda^{\prime \prime} \ln \left(\frac{x}{x_{0}}\right) \nu^{2}+i \nu \ln k^{2}+\left.i \nu \frac{\partial \ln \tilde{f}}{\partial \nu}\right|_{\nu=0}\right\} \\
& =\frac{1}{2 \pi}\left(k^{2}\right)^{\frac{1}{2}} \tilde{f}\left(x_{0}, \frac{1}{2}\right)\left(\frac{x}{x_{0}}\right)^{-\lambda} \int_{-\infty}^{+\infty} d \nu \exp \left\{\frac{1}{2} \lambda^{\prime \prime}\left(\frac{x}{x_{0}}\right) \nu^{2}+i \nu \ln k^{2}+\left.\nu \frac{\partial \ln \tilde{f}}{\partial \nu}\right|_{\nu=0}\right\}
\end{aligned}
$$


Assumindo:

$$
\ln \left(\bar{k}^{2}\right)=\left.i \frac{\partial}{\partial \nu} \ln \tilde{f}\left(x_{0}\right)\right|_{\nu=0}
$$

$\mathrm{e}$

$$
i \nu \ln \left(\frac{1}{\bar{k}^{2}}\right)=\left.\nu \frac{\partial}{\partial \nu} \ln \tilde{f}\left(x_{0}\right)\right|_{\nu=0}
$$

Assim ficamos com:

$$
f\left(x, k^{2}\right)=\frac{1}{2 \pi}\left(k^{2}\right)^{\frac{1}{2}} \tilde{f}\left(x_{0}, \frac{1}{2}\right)\left(\frac{x}{x_{0}}\right)^{-\lambda} \int_{-\infty}^{+\infty} d \nu \exp \left\{-\frac{1}{2} \lambda^{\prime \prime} \ln \left(\frac{x_{0}}{x}\right) \nu^{2}+i \nu \ln \left(\frac{k^{2}}{\bar{k}^{2}}\right)\right\}
$$

Onde identificamos uma integral gaussiana. Assim

$$
\begin{gathered}
f\left(x, k^{2}\right)=\frac{1}{2 \pi}\left(k^{2}\right)^{\frac{1}{2}} \tilde{f}\left(x_{0}, \frac{1}{2}\right)\left(\frac{x}{x_{0}}\right)^{-\lambda} \sqrt{\frac{\pi}{\frac{1}{2} \lambda^{\prime \prime} \ln \frac{x_{0}}{x}}} \exp \left[-\frac{\ln ^{2}\left(\frac{k^{2}}{\bar{k}^{2}}\right)}{2 \lambda^{\prime \prime} \ln \frac{x_{0}}{x}}\right] \\
\frac{f\left(x, k^{2}\right)}{\left(k^{2}\right)^{\frac{1}{2}}} \sim\left(\frac{x}{x_{0}}\right)^{\lambda} \frac{1}{\left(\ln \frac{x_{0}}{x}\right)^{\frac{1}{2}}} \exp \left[-\frac{\ln ^{2}\left(\frac{k^{2}}{\bar{k}^{2}}\right)}{2 \lambda^{\prime \prime} \ln \frac{x_{0}}{x}}\right]
\end{gathered}
$$

onde o argumento da exponencial é o termo de difusão. A falta de ordenamento nos momentos transversais da escada gluônica é a origem da difusão. Note que esse termo de difusão não está presente na solução da equação de DGLAP, pois esta tem um forte ordenamento nos momentos transversais.

\section{Dimensão anômala}

A fim de obtermos a dimensão anômala da BFKL, aplicamos a transformada de Mellin em ambos os lados da equação $(0,0.7)$ :

$$
-\int_{0}^{1} d x x^{N-1} x \frac{\partial \tilde{f}}{\partial x}=\tilde{k}(\gamma) \int_{0}^{1} d x x^{N-1} \tilde{f}(x, \gamma)
$$

como

$$
\int_{0}^{1} d x x^{N-1} \tilde{f}(x, \gamma)=F(N, \gamma)
$$


escrevemos

$$
-\int_{0}^{1} d x x^{N} \frac{\partial \tilde{f}}{\partial x}=\tilde{k}(\gamma) F(N, \gamma)
$$

Usando o fato de que

$$
\frac{d}{d x}\left(x^{N} f\right)=N x^{N-1} f+x^{N} \frac{\partial f}{\partial x}
$$

podemos escrever a equação acima:

$$
\begin{aligned}
\int_{0}^{1} d x x^{N} \frac{\partial \tilde{f}}{\partial x} & =\int_{0}^{1} d x \frac{d}{d x}\left(x^{N} \tilde{f}\right)-\int_{0}^{1} N x^{N-1} \tilde{f} d x \\
& =\left.x^{N} \tilde{f}\right|_{0} ^{1}-N \int_{0}^{1} x^{N-1} \tilde{f} d x \\
& =\left.x^{N} \tilde{f}\right|_{0} ^{1}-N F(N, \gamma) .
\end{aligned}
$$

Voltando em $(0,0.17)$ temos

$$
\left.x^{N} \tilde{f}\right|_{0} ^{1}-N F(N, \gamma)=-\tilde{k}(\gamma) F(N, \gamma)
$$

finalmente obtemos para $F(N, \gamma)$ :

$$
F(N, \gamma)=\frac{\tilde{f}^{(0)}}{1-\frac{1}{N} \tilde{k}(\gamma)}
$$

A partir da expressão acima vemos que o comportamento da distribuição de gluons não integrada é controlada pelo pólo:

$$
1-\frac{1}{N} \tilde{k}(\gamma)=0
$$

onde $\tilde{k}(\gamma)$ é o kernel da BFKL. Usando a expansão de $\tilde{k}$ dada em $(0,0.12)$ obtemos:

$$
1-\frac{1}{N} \bar{\alpha}_{s}\left[4 \log 2+14 \zeta(3)(\gamma-1 / 2)^{2}+\ldots\right]=0
$$


onde $\bar{\alpha}_{s} \equiv \frac{3 \alpha_{s}}{\pi}$. Assim

$$
\begin{gathered}
\frac{\bar{\alpha}_{s}}{N}\left[4 \log 2+14 \zeta(3)(\gamma-1 / 2)^{2}+\ldots\right]=1 \\
4 \log 2+14 \zeta(3)(\gamma-1 / 2)^{2}=\frac{N}{\bar{\alpha}_{s}}
\end{gathered}
$$

Resolvendo para $\gamma$ teremos:

$$
\gamma=\frac{1}{2} \pm \sqrt{\frac{N-\bar{\alpha}_{s} 4 \log 2}{14 \bar{\alpha}_{s} \zeta(3)}}
$$

onde iremos identificar o termo $N_{L}$ como $N_{L}=\bar{\alpha}_{s} 4 \log 2$, $\operatorname{logo}$

$$
\gamma=\frac{1}{2} \pm \sqrt{\frac{N-N_{L}}{14 \bar{\alpha}_{s} \zeta(3)}}
$$




\section{Referências Bibliográficas}

[1] M. S. Kugeratski and F. S. Navarra, Phys. Rev. C71, 065206 (2005).

[2] D.A. Fogaça, M.S. Kugeratski and F.S. Navarra, Braz. Jour. Phys. 34 , 276 (2004).

[3] D. D. Dietrich, G. C. Nayak and W. Greiner, Phys. Rev. D64, 074006 (2001).

[4] L. D. McLerran and R. Venugopalan, Phys. Rev. D 49, 2233 (1994) [arXiv:hep$\mathrm{ph} / 9309289]$.

[5] L. D. McLerran and R. Venugopalan, Phys. Rev. D 49, 3352 (1994) [arXiv:hep$\mathrm{ph} / 9311205]$.

[6] M. S. Kugeratski and F. S. Navarra, Proceedings: "IX Hadron Physics and VII Relativistic Aspects of Nuclear Physics: Joint Meeting on QCD/QGP", AIP Conf .Proc. 739:566, 2005. Angra dos Reis, 566 (2004).

[7] M. S. Kugeratski, V. P. Gonçalves and F. S. Navarra, Eur. Phys. J. C44, 577 (2005).

[8] V. P. Gonçalves, M. S. Kugeratski, M. V. T. Machado and F. S. Navarra, Phys. Lett. B643, 273 (2006).

[9] M. S. Kugeratski, V. P. Gonçalves and F. S. Navarra, Eur. Phys. J. C46, 413 (2006). 
[10] M. S. Kugeratski, V. P. Gonçalves and F. S. Navarra, Eur. Phys. J. C46, 465 (2006).

[11] T. Muta, Foundations of Quantum Chromodynamics, World Scientific (1987).

[12] F. Halzen and A. D. Martin, Quarks and Leptons: An Introductory Course in Modern Particle Physics, John Wiley \& Sons (1984), página 184.

[13] J.D. Bjorken, Phys. Rev. 179 (1969) 1547.

[14] R.P. Feynman, Phys. Rev. Lett. 23 (1969) 1415.

[15] R.P. Feynman, Photon Hadron Interactions, W.A. Benjamin, New York, 1972.

[16] J.D. Bjorken and E.A. Paschos, Phys. Rev. 185 (1969) 1975.

[17] W. Greiner and A. Schafer, Quantum Chromodynamics, Springer (1994).

[18] H. L. Lai et al. [CTEQ Collaboration], Eur. Phys. J. C 12, 375 (2000).

[19] V. P. Gonçalves, A Cromodinâmica Quântica para sistemas densos. Porto Alegre: Instituto de Física da UFRGS, 2000. Tese de Doutorado.

[20] L.V. Gribov, E.M. Levin and M.G. Ryskin, Phys. Rep. 100, 1 (1983).

[21] E. Iancu e R. Venugopalan, hep-ph/0303204. Publicado em QGP3, Eds. R.C. Hwa and X.N.Wang, World Scientific; E. Iancu, A. Leonidov and L. McLerran, The Color Glass Condensate: An introduction, hep-ph/0202270, publicado em QCD Perspectives on Hot and Dense Matter, Eds. J. -P. Blaizot and E. Iancu, NATO Science Series, Kluwer, 2002; E. Iancu and R, Venugopalan, The Color Glass Condensate and High Energy Scattering in QCD, hep-ph/0303104; J. JalilianMarian,J. Phys. G30, 751 (2004);

[22] J. Jalilian-Marian, A. Kovner, A. Leonidov and H. Weigert, Nucl. Phys. B504, 415 (1997); Phys. Rev. D59, 014014 (1999); J. Jalilian-Marian, A. Kovner and 
H. Weigert, Phys. Rev. D59, 014015 (1999); H. Weigert, Nucl. Phys. A703, 823 (2002).

[23] A. L. Ayala Filho, Formalismo de Glauber para interações partônicas. Porto Alegre: Instituto de Física da UFGRS, 1998. Tese de Doutorado.

[24] A. L. Ayala Filho, M. B. Gay Ducati and E. M. Levin, Nucl. Phys. B493, 305 (1997), ibid. B511, 355 (1998).

[25] A. L. V. Ramos dos Reis, Saturação de gluons e perda de energia em colisões hadrônicas. São Paulo, USP, 2006. Dissertação de Mestrado.

[26] K. Golec-Biernat and M. Wüsthoff, Phys. Rev. D 59, 014017 (1999), ibid. D60 114023 (1999).

[27] L. McLerran, arXiv:hep-ph/0402137.

[28] G. Ingelman and P. Schlein, Phys. Lett. B152, 256 (1985).

[29] V. Barone and E.Predazzi, High-Energy Particle Diffraction, Springer-Verlag, Berlin Heidelberg (2002).

[30] N. N. Nikolaev and B. G. Zakharov, Z. Phys. C49, 607 (1991); Z. Phys. C53, 331 (1992); A. H. Mueller, Nucl. Phys. B415, 373 (1994); A. H. Mueller and B. Patel, Nucl. Phys. B425, 471 (1994).

[31] K. Golec-Biernat, Deep inelastic scattering at small values of the Bjorken variable $x$. Kraków: Institute of Nuclear Physics, 2001. Habilitation Thesis.

[32] G. R. Kerley, Modelling the diffractive interaction of the photon. The University of Manchester, 2000. PhD Thesis.

[33] M. V. T. Machado, Física de Pomeron a altas energias . Porto Alegre: Instituto de Física da UFRGS, 2004. Tese de Doutorado.

[34] M. Wusthoff, Phys. Rev. D 56 (1997) 4311 
[35] K. Golec-Biernat and M. Wüsthoff, Phys. Rev. D60, 114023 (1999).

[36] J. R. Forshaw, R. Sandapen and G. Shaw, Phys. Lett. B 594, 283 (2004).

[37] J. R. Forshaw and D. A. Ross, Quantum Chromodynamics and the Pomeron, Cambridge University Press (1997).

[38] V.N. Gribov and L.N. Lipatov, Sov. J. Nucl. Phys. 15, 438 (1972); G. Altarelli and G. Parisi, Nucl. Phys. B126, 298 (1977); Yu.L. Dokshitzer, Sov. Phys. JETP 46, 641 (1977).

[39] L. N. Lipatov, Sov. J. Nucl. Phys. 23, 338 (1976); E. A. Kuraev, L. N. Lipatov, V. S. Fadin, JETP 45, 1999 (1977); I. I. Balitskii, L. N. Lipatov, Sov. J. Nucl. Phys. 28, 822 (1978).

[40] A.H. Mueller and Jian-wei Qiu, Nucl. Phys. B268, 427 (1986).

[41] A. M. Staśto, K. Golec-Biernat and J. Kwieciński, Phys. Rev. Lett. 86, 596 (2001).

[42] J. L. Albacete, N. Armesto, J. G. Milhano, C. A. Salgado and U. A. Wiedemann, Phys, Rev. D71, 014003 (2005).

[43] I. I. Balitsky, Nucl. Phys. B463 (1996) 99; Y.V. Kovchegov, Phys. Rev. D 60 (1999) 034008.

[44] A. M. Stasto, Acta Phys. Polon. B 35, 3069 (2004).

[45] E. Levin and K. Tuchin, Nucl. Phys. B 573, 833 (2000).

[46] E. Iancu, K. Itakura and L. McLerran, Nucl. Phys A708, 327 (2002).

[47] I. I. Balitsky, Nucl. Phys. B463, 99 (1996), Phys. Rev. Lett. 81, 2024 (1998), Phys. Rev. D 60, 014020 (1999), Phys. Lett. B 518, 235 (2001); I.I. Balitsky and A.V. Belitsky, Nucl. Phys. B629, 290 (2002). 
[48] E. Iancu, A. Leonidov and L. McLerran, Nucl.Phys. A692 (2001) 583; E. Ferreiro, E. Iancu, A. Leonidov and L. McLerran, Nucl. Phys. A701, 489 (2002).

[49] J. Jalilian-Marian, A. Kovner, L. McLerran and H. Weigert, Phys. Rev. D 55, 5414 (1997); J. Jalilian-Marian, A. Kovner and H. Weigert, Phys. Rev. D 59, 014014 (1999), ibid. 59, 014015 (1999), ibid. 59034007 (1999); A. Kovner, J. Guilherme Milhano and H. Weigert, Phys. Rev. D 62, 114005 (2000); H. Weigert, Nucl. Phys. A703, 823 (2002).

[50] E. Iancu, K. Itakura, S. Munier, Phys. Lett. B590, 199 (2004).

[51] A.H. Mueller and D.N. Triantafyllopoulos, Nucl. Phys. B640, 331 (2002); D.N. Triantafyllopoulos, Nucl. Phys. B648, 293 (2003); A.H. Mueller, Nucl. Phys. A724, 223 (2003).

[52] E. Gotsman, E. Levin, M. Lublinsky and U. Maor, Eur. Phys. J. C 27, 411 (2003)

[53] J. R. Forshaw, R. Sandapen and G. Shaw, Phys. Rev. D 69, 094013 (2004).

[54] V. P. Gonçalves and M. V. T. Machado, Eur. Phys. J. C 37, 299 (2004)

[55] D. Kharzeev, Y.V. Kovchegov and K. Tuchin, Phys. Lett. B599, 23 (2004).

[56] A. Dumitru, A. Hayashigaki and J. Jalilian-Marian, Nucl. Phys. A 765, 464 (2006); Nucl. Phys. A 770, 57 (2006).

[57] J. R. Forshaw, G. Kerley and G. Shaw, Phys. Rev. D 60, 074012 (1999); J. R. Forshaw, G. R. Kerley and G. Shaw, Nucl. Phys. A 675, 80C (2000).

[58] S. Chekanov et al. [ZEUS Collaboration], Eur. Phys. J. C 21, 443 (2001)

[59] C. Adloff et al. [H1 Collaboration], Eur. Phys. J. C 21, 33 (2001); E. M. Lobodzinska, Acta Phys. Polon. B 35 (2004) 223; arXiv:hep-ph/0311180.

[60] M. V. T. Machado, Eur. Phys. J. C 47, 365 (2006). 
[61] B. A. Kniehl, G. Kramer and B. Potter, Nucl. Phys. B 582, 514 (2000).

[62] I. Arsene et al. [BRAHMS Collaboration], Phys. Rev. Lett. 91, 072305 (2003); Phys. Rev. Lett. 93, 242303 (2004); Phys. Rev. Lett. 94, 032301 (2005); arXiv:nucl-ex/0410020.

[63] A. Despande, R. Milner, R. Venugopalan and W. Vogelsang, Ann. Rev. Nucl. Part. Sci. 55 (2005) 165.

[64] K. Prytz, Phys. Lett. B 311, 286 (1993); A. J. Askew, K. Golec-Biernat, J. Kwiecinski, A. D. Martin and P. J. Sutton, Phys. Lett. B 325, 212 (1994); R. K. Ellis, Z. Kunszt and E. M. Levin, Nucl. Phys. B 420, 517 (1994) [Erratum-ibid. B 433, 498 (1995)]; M. B. Gay Ducati and V. P. Gonçalves, Phys. Lett. B 390, 401 (1997).

[65] A. L. Ayala, M. B. Gay Ducati and E. M. Levin, Phys. Lett. B 388, 188 (1996)

[66] V. P. Gonçalves, Phys. Lett. B 495, 303 (2000)

[67] N. N. Nikolaev, B. G. Zakharov and V. R. Zoller, Z. Phys. A 351 (1995) 435.

[68] Y. V. Kovchegov and L. D. McLerran, Phys. Rev. D 60 (1999) 054025 [Erratumibid. D 62 (2000) 019901]

[69] E. Gotsman, E. Levin, M. Lublinsky, U. Maor and K. Tuchin, Phys. Lett. B 492 (2000) 47

[70] E. Gotsman, E. Levin, U. Maor, L. D. McLerran and K. Tuchin, Nucl. Phys. A 683, $383(2001)$

[71] E. Levin and M. Lublinsky, Nucl. Phys. A 712 (2002) 95.

[72] L. Frankfurt, V. Guzey, M. Strikman, Phys. Lett. B 586 (2004) 41.

[73] S. Munier and A. Shoshi, Phys. Rev. D 69 (2004) 074022 
[74] V. P. Gonçalves and M. V. T. Machado, Eur. Phys. J. C 30, 387 (2003)

[75] V. P. Gonçalves and M. V. T. Machado, Eur. Phys. J. C 38, 319 (2004)

[76] Arneodo M, et al. In: Proceedings of "Future Physics at HERA", DESY, September 1995, arXiv:hep-ph/9610423.

[77] H. Abramowicz, L. Frankfurt and M. Strikman, Surveys High Energ. Phys. 11 (1997) 51.

[78] J. Breitweg et al. [ZEUS Collaboration], Eur. Phys. J. C6 (1999) 43; S. Chekanov et al. [ZEUS Collaboration], Eur. Phys. J. C38 (2004) 43; C. Adloff et al. [H1 Collaboration], Z. Phys. C 76 (1997) 613.

[79] Para uma revisão recente, veja F.O. Durães et al., Phys. Rev. C68, 035208 (2003).

[80] C.-Y. Wong, E. S. Swanson and T. Barnes, Phys. Rev. C62, 045201 (2000); C65, 014903 (2001).

[81] Y. Oh, T. Song and S.H. Lee, Phys. Rev. C63, 034901 (2001).

[82] F.S. Navarra et al., Phys. Lett. B 529, 87 (2002); M. Nielsen et al., Braz. Jour. Phys. 33 , 316 (2003); F.O. Durães et al., Phys. Lett. B564, 97 (2003).

[83] M.E. Peskin, Nucl. Phys. B156, 365 (1979) ; G. Bhanot and M.E. Peskin, Nucl. Phys. B156, 391 (1979).

[84] D.Kharzeev and H. Satz, Phys. Lett. B366, 316 (1996); B356, 365 (1995); B334, 155 (1994).

[85] D. Kharzeev, nucl-th/9601029.

[86] F. Arleo, P.B. Gossiaux, T. Gousset and J. Aichelin, Phys. Rev. C65, 014005 (2002) .

[87] Y. Oh, S. Kim and S.H. Lee, Phys. Rev. C65 067901 (2002). 
[88] Taesoo Song and Su Houng Lee, hep-ph/0501252.

[89] L. Gerland et al., Phys. Rev. Lett. 81, 762 (1998).

[90] H.G. Dosch, F.S. Navarra, M. Nielsen and M. Rueter, Phys. Lett. B466, 363 (1999).

[91] J. Hüfner, Y.P. Ivanov, B.Z. Kopeliovich and A.V. Tarasov, Phys. Rev. D62, 094022 (2000).

[92] B. Müller, nucl-th/9806023; S.C. Benzahra, Phys. Rev. C61, 064906 (2000).

[93] M. Rueter and H.G. Dosch, Z. Phys. C66, 245 (1995).

[94] T.T. Takahashi, H. Matsufuru, Y. Nemoto and H. Suganuma, Phys. Rev. Lett. 86, 18 (2001); Phys. Rev. D65, 114509 (2002); T.T. Takahashi and H. Suganuma, Phys. Rev. Lett. 90, 182001 (2003).

[95] V.Kuzmenko and Y.A. Simonov, Phys. Lett. B494, 81 (2000); V. Kuzmenko, hep-ph/0204250.

[96] P.O. Bowman and A.P. Szczepaniak, Phys. Rev. D70, 016002 (2004).

[97] Estamos usando a notação introduzida em D. Griffiths, Introduction to Elementary Particles, J. Wiley and Sons, 1987, Capítulo 9.

[98] J. Vijande, F. Fernandez, A. Valcarce, B. Silvestre-Brac, Eur. Phys. J. A19, 383 (2004); S. Godfrey and N. Isgur, Phys. Rev. D32, 189 (1985). 